\title{
Multiquark Resonances
}

\author{
A. Esposito ${ }^{\text {a }}$ A. Pilloni ${ }^{\text {b }}$ A. D. Polosa ${ }^{\mathrm{c}, \mathrm{d}}$ \\ ${ }^{a}$ Physics Department, Center for Theoretical Physics E Institute for Strings, Cosmology, and Astroparticle Physics, \\ Columbia University, New York, NY 10027, USA \\ ${ }^{b}$ Theory Center, Thomas Jefferson National Accelerator Facility, 12000 Jefferson Avenue, Newport News, VA 23606, USA \\ cDipartimento di Fisica and INFN, “Sapienza” Università di Roma, P.le A. Moro 2, I-00185 Roma, Italy \\ ${ }^{d}$ CERN, Theory Department, Geneva 1211, Switzerland
}

\begin{abstract}
Multiquark resonances are undoubtedly experimentally observed. The number of states and the amount of details on their properties has been growing over the years. It is very recent the discovery of two pentaquarks and the confirmation of four tetraquarks, two of which had not been observed before. We mainly review the theoretical understanding of this sector of particle physics phenomenology and present some considerations attempting a coherent description of the so called $X$ and $Z$ resonances. The prominent problems plaguing theoretical models, like the absence of selection rules limiting the number of states predicted, motivate new directions in model building. Data are reviewed going through all of the observed resonances with particular attention to their common features and the purpose of providing a starting point to further research.
\end{abstract}

Keywords: Tetraquarks, Pentaquarks, Exotic Hadron Resonances

PACS: 14.40.Rt, 14.20.Pt, 12.40.-y, 12.39.Jh

\section{Contents}

1 Introduction

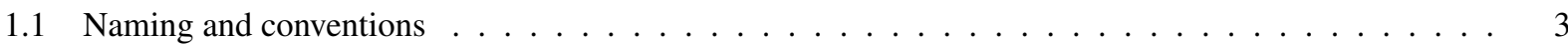

1.2 A very brief survey of the experimental picture . . . . . . . . . . . . . . . 4

2 Scattering in presence of shallow bound states 5

2.1 Shallow bound states: the phase shift from wave mechanics . . . . . . . . . . . . . . . . . . 6

2.2 Shallow bound states: the phase shift from scattering theory $\ldots \ldots \ldots \ldots$

3 Loosely bound molecules

Preprint submitted to Elsevier

November 14, 2016

(C) 2016. This manuscript version is made available under the Elsevier user license http://www.elsevier.com/open-access/userlicense/1.0/ 
3.1 Relation between binding energy and the decay rate of $X(3872) \ldots \ldots \ldots \ldots$

3.2 Scattering length of the $X(3872)$ as a loosely bound molecule $\ldots \ldots \ldots \ldots$

$3.3 \quad X(3872)$ as a deuteron-like state $\ldots \ldots \ldots \ldots \ldots \ldots \ldots$

3.4 Consequences of the Heavy Quark Spin Symmetry . . . . . . . . . . . . . . . . . . . . . . . . . . 20

3.5 An effective field theory approach to heavy meson molecules . . . . . . . . . . . . . . . . 21

3.6 Phenomenological realizations of the meson-meson potential . . . . . . . . . . . . . 23

3.7 Open problems with the molecular model f . . . . . . . . . . . . . . . . . . . . . 27

4 Diquark building blocks $\quad 28$

4.1 General features . . . . . . . . . . . . . . . . . . . . . . . . . . . . . 29

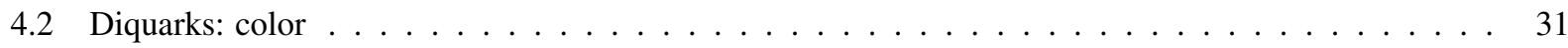

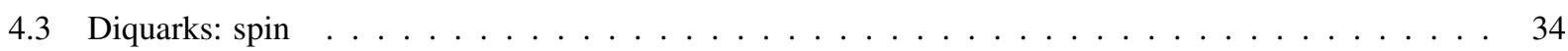

4.4 Spectrum for $L=0$ and the $X, Z, Z^{\prime}$ system $\ldots \ldots \ldots \ldots \ldots \ldots$

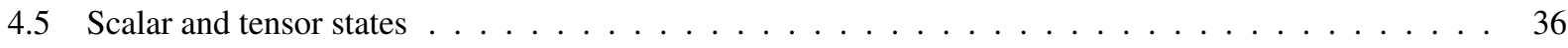

$4.6 Y$ resonances and $L=1$ diquarkonia $\ldots \ldots \ldots \ldots \ldots \ldots \ldots$

4.7 Pentaquarks from diquarks $\ldots \ldots \ldots \ldots \ldots \ldots \ldots$

5 Tetraquarks and diquarks in the $1 / N$ expansion 42

$5.11 / N$ expansion in QCD: a short reminder . . . . . . . . . . . . . . . . . . 43

5.2 Tetraquarks in the $1 / N$ expansion $\ldots \ldots \ldots \ldots \ldots \ldots \ldots \ldots$

5.3 Diquarkonia in the $1 / N$ expansion $\ldots \ldots \ldots \ldots \ldots \ldots \ldots$

5.4 Further remarks $\ldots \ldots \ldots \ldots \ldots \ldots \ldots \ldots \ldots \ldots$

6 An alternative picture of $X Z$ resonances $\quad 55$

6.1 The discrete spectrum of diquarkonia in the continuum of free meson-meson states $\ldots \ldots \ldots 5$

$6.2 \Psi_{m}$ and $\Psi_{d}$ states $\ldots \ldots \ldots \ldots \ldots \ldots \ldots \ldots \ldots$

6.3 The $X_{b}$ in the beauty sector, isospin violations and the non-observation of $X^{+} \ldots \ldots$ 
6.4 The special case of the $X(4140) \ldots \ldots \ldots \ldots \ldots \ldots$

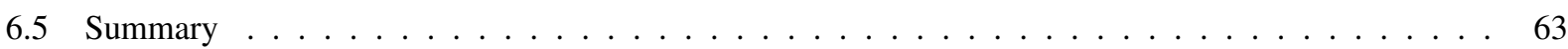

7 Other models and approaches $\quad 64$

7.1 Hadroquarkonium . . . . . . . . . . . . . . . . . . . . . . . 64

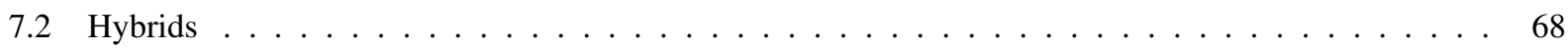

7.3 Nothing: kinematical effects . . . . . . . . . . . . . . . . . . . . . . 73

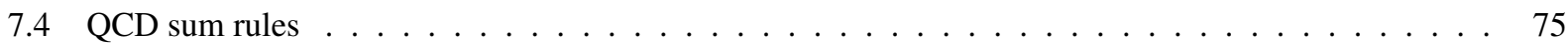

7.5 Lattice QCD . . . . . . . . . . . . . . . . . . . . . . . . . 77

8 A travel guide to experimental results $\quad 79$

8.1 The $X(3872) \ldots \ldots \ldots \ldots \ldots \ldots \ldots \ldots$

8.2 The $Z_{c}(3900)$ and $Z_{c}^{\prime}(4020) \ldots \ldots \ldots \ldots \ldots \ldots \ldots \ldots$

8.3 The bottomonium system: $Z_{b}(10610)$ and $Z_{b}^{\prime}(10650)$, and search for $X_{b}$ and $Y_{b} \ldots \ldots$

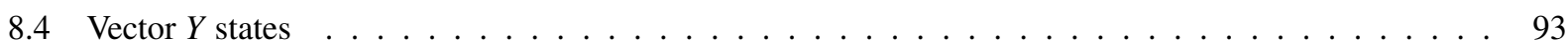

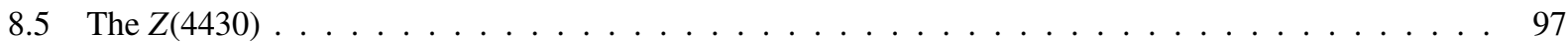

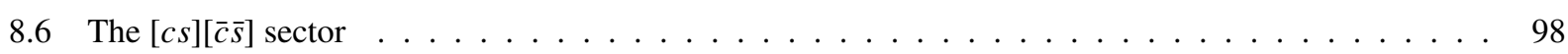

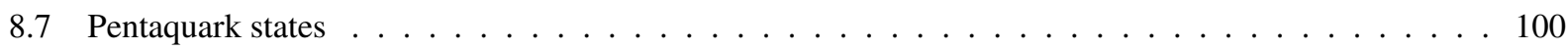

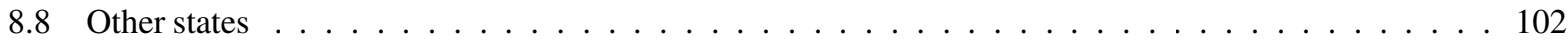

9 Conclusions and outlook $\quad 104$

$\begin{array}{lll}\text { Appendix A } & X(3872) \text { production at hadron colliders } & 106\end{array}$

$\begin{array}{lll}\text { Appendix B } & \text { Diquarks in SU(N) } & 109\end{array}$

Appendix C Interaction Hamiltonian between open and closed channels in strong interactions 112

$\begin{array}{lll}\text { Appendix D } & \text { The effective hadroquarkonium Hamiltonian } & 113\end{array}$ 


\section{Introduction}

Yet another review on tetraquarks, pentaquarks, and all that?

Indeed this paper comes after a number of encyclopædic reviews on the theme appeared since the discovery of the $X(3872)$ - see [1-5] and the latest, very comprehensive [6].

Despite the considerable amount of information provided by these articles, what presented here is a further attempt to report more specifically on those efforts made to find simple theoretical descriptions, even though incomplete, which include and explain, in a unitary picture, most of the exotic resonances observed at the time of this writing. Along these lines, some new arguments and work in progress will also be presented.

We assume that there is a common theoretical description of the $X^{0}(3872), Z_{c}^{0, \pm}(3900), Z_{c}^{\prime 0, \pm}(4020), X^{0}(4140)$, $Z_{b}^{0, \pm}(10610), Z_{b}^{\prime 0, \pm}(10650), Z(4430)$ and the pentaquarks, and we collect those ideas which appear to us to be functional to formulate such a comprehensive picture, even if it cannot yet be considered as complete and satisfactory.

The non-observation of $X^{ \pm}(3872)$, the isospin violating decay pattern of $X^{0}$, the absence of $X^{0, \pm}$ partners in the beauty sector, which challenge the compact tetraquark interpretation [7,8], are taken as starting points of our analysis ${ }^{1}$. On the other hand, an obvious merit of compact tetraquark models was that of strongly motivating the research on charged resonances, which were eventually copiously observed, contrary to most expectations.

The evident proximity of meson-meson thresholds to the observed states, which appears as an impressive finetuning in the case of the $X^{0}(3872)$, is also taken as a serious indication which, in the light of available data, seems unlikely to be accidental.

Meson molecule models have the same problem with the proliferation of states, despite of the attempts made to solve it. Moreover they have definitive problems at explaining prompt production at hadron colliders, especially in the case of the $X$. Bona fide molecules like the deuteron are observed at hadron colliders, but only at very low transverse momenta, contrary to the $X$, which is detected at extremely large transverse momenta $\left(p_{\perp} \gtrsim 15 \mathrm{GeV}\right)$. These are very serious features, often forgotten, which should not be ignored.

Because of these and other reasons, explained with more details in the text, we might conclude that none of these two models reaches a fully satisfactory or definitive description of what observed in experiment, even though we must recognize a certain degree of success of both of them at explaining the features of some specific resonances.

On the other hand, we are convinced that diquark degrees of freedom are essential for the construction of a theory of multiquark resonances and that most of the work done in refining the predictions of the diquarkonium picture must be part of it.

What has been missing in the diquarkonium model are sort of 'selection rules' explaining the paucity of states which have been observed over the years. Same for the hadron molecules. Together with the presentation of hadron molecule and diquark-antidiquark models, we collect here some ideas we have been working on, which never appeared in an extensive discussion, to show one possible way to describe $X$ and $Z$ resonances as the result of an effective interaction in meson-meson channels leading to the formation of resonant metastable states. This effective interaction is due to the presence of the discrete spectrum of diquarkonia immersed in the continuum spectrum of would-be molecules.

Special conditions are needed to switch on this interaction, but once they are met, sharp resonances are expected to

\footnotetext{
${ }^{1}$ We assume here that the experimental situation is definitive on these matters, being understood that any variation on these data, would revolutionize our understanding of multiquark resonances.
} 
be produced. These conditions strongly restrict the number of possible states and we have used the best experimentally assessed tetraquark resonance to test the picture. This discussion is presented in Section 6 and the arguments there formulated are admittedly not yet fully satisfactory, although showing rather suggestive aspects.

The paper is organized as follows.

We first analyze the core of ideas and methods at the basis of meson molecule models, starting from those textbook results on low energy scattering which we found useful to set the frame for the arguments to be developed. We criticize the limits of the hadron molecule approach, and discuss just some of the various technical solutions employed. This is done in Sections 2 and 3.

In Section 4 we present the diquarkonium model and the methods developed to obtain a spectrum of states which remarkably reproduces the $X, Z, Z^{\prime}$ mass pattern. The limitation of this model resides in the number of states predicted which, at the time of this writing, have not been observed. If in some future the experimental picture will be revolutionized by the appearance of the large number of expected resonances, together with $X^{ \pm}$states (which mysteriously evaded observation until now) etc., the diquarkonium model alone will certainly be, in our view, the strongest and most grounded option to describe this physics.

A more theoretical discussion of tetraquarks in the $1 / N$ expansion [9] adds confidence on the specific role of diquarks as the right degrees of freedom to treat the emergence of tetraquark poles in meson-meson amplitudes. This is discussed in Section 5, which reproduces some results recently obtained.

This review might be read as a standalone research paper by skipping the first five sections. Section 6 stems from some work initiated in [10] and especially in [11]. However incomplete it might appear, the approach presented appears to us as one of the possible routes which should be explored to overcome the phenomenological problems of $X Y Z$ resonances.

In addition to this, in Section 7 we have presented known results on some interesting approaches, which are needed to offer a coherent picture of the work done in the field, going beyond the molecule picture of hadrons held together by nuclear forces. This is also done with the purpose of underscoring the efforts towards a 'unified' approach to the description of the observed phenomenology.

A report on the experimental situation can be found in the last part of the review, Section 8. We go through resonance by resonance, referring to the theoretical interpretations presented in the paper and highlighting connections and common features. The aim is always that of finding more or less hidden connections among the observed resonances. This part is intended to be a guide to the existing spectroscopy, especially meant for 'model-building' purposes. We omit a number of details which have been presented in other reviews.

The discussion on pentaquarks is limited to a brief report on what has already been done, especially in the diquark model. Pentaquarks were, before their discovery, a highly undesirable option for hadron molecules. If new resonances with tetraquark or exaquark quantum numbers will be discovered, and if the approach described in Section 6 will resist to new forthcoming data on tetraquarks, it might straightforwardly be applied to more complex hadron structures. The rules for doing these steps are explained, as we understand them at the moment.

Most likely to explain the nature of $X Y Z P$ resonances does not require 'new physics', in the most common adopted meaning, but likely some conceptual leap in the use of 'old' strong interaction dynamics. We also do not think that $X Y Z$ resonances are sort of nuclear chemistry phenomena as they also occur, as witnessed by the prompt production of the $X$, at very large transverse momentum.

We hope to offer in this paper some perspective on the field and hopefully a support to identify new research directions. 


\subsection{Naming and conventions}

There is some confusion about the naming of the resonances. At the beginning, the letters $X, Y$ and $Z$ were used with no clear criteria. The PDG decided to call $X$ (mass in $\mathrm{MeV}$ ) all the exotic quarkoniumlike states, with the exception of the $\chi_{c 0}(3915)$ (initially $X(3915)$, then promoted to the ordinary charmonium name $\chi_{c 0}(2 P)$ in the PDG 2014 , now assessed). However, a sort of convention is generally followed in the literature, which calls $Z$ (mass) the charged quarkoniumlike states, $Y$ (mass) the vector $1^{--}$ones, $P$ (mass) the pentaquarks, and $X$ (mass) all the other ones. We will follow this convention, with the addition of the superscripts and subscripts used by Belle and BES III, to wit $Z_{c}, Z_{c}^{\prime}, Z_{b}, Z_{b}^{\prime}, Z_{1}, Z_{2}$, as well as $P_{c}$ for the hidden charm pentaquarks. We call $X(4140)$ the state seen in $J / \psi \phi$ (known in the past as $Y(4140)), \chi_{c 0}(3915)$ the state seen in $J / \psi \omega$ (known in the past as $X(3915), Y(3915)$, or $\left.Y(3940)\right), Z(4430)$ the state seen in $\psi(2 S) \pi$ (sometimes called $Z(4475)$ ).

The charged conjugated modes are always understood, unless specified. The convention for the phases is such that $C\left|D^{0}\right\rangle=\left|\bar{D}^{0}\right\rangle$, while $C\left|D^{* 0}\right\rangle=-\left|\bar{D}^{* 0}\right\rangle$. The reader has to pay attention to different conventions used in the literature.

With a little abuse of notation, we will talk of $C$-parity for charged states, meaning "the $C$-parity eigenvalue of the neutral isospin partner".

We do not discuss the former $Z(3930)$ and the $X(3823)$, which are good candidates for the ordinary charmonia $\chi_{c 2}(2 P)$ and $\psi_{2}(1 D)$, respectively.

\subsection{A very brief survey of the experimental picture}

A detailed analysis of the experimental status of multiquark resonances will be presented in the last Section of this report, together with their theoretical interpretations and references to experimental analyses.

Here we shall briefly review the basic experimental facts about the most compelling $X Y Z$ resonances to set the stage for the discussion to follow.

The first clearly exotic multiquark resonance is the Z(4430), first claimed by Belle in 2007, but confirmed only in 2014 by the LHCb collaboration. It was observed in the

$$
\bar{B}^{0} \rightarrow K^{-}\left(\psi(2 S) \pi^{+}\right)
$$

channel, i.e. $Z(4430) \rightarrow \psi(2 S) \pi^{+}$. The $s$ quark from the weak $b \rightarrow c \bar{c} s$ transition makes a $K^{-}$with a $\bar{u}$ quark from a vacuum $u \bar{u}$ pair. The remaining $u$ and $\bar{d}$ quarks, together with the $c \bar{c}$ pair, constitute the valence of the $\left(\psi(2 S) \pi^{+}\right)$ resonance. How does hadronization work in this process? Is the $\left(\psi(2 S) \pi^{+}\right)$resonance, otherwise dubbed $Z(4430)$, a compact four-quark hadron, like a baryon or a meson, but with a different quark skeleton? Or is it just a hadron molecule kept bound for a finite lifetime by long range residual strong interactions?

In 2013 another resonance, the $Z_{c}(3900)$, was observed simultaneously by BES III and Belle, as a decay product of the $Y(4260)$

$$
Y(4260) \rightarrow \pi^{+}\left(J / \psi \pi^{-}\right)
$$

with $Y(4260)$ being a $J^{P C}=1^{--}$neutral state, produced in initial state radiation in $e^{+} e^{-}$collisions. $Y(4260)$ is also a multiquark resonance candidate, albeit neutral, with a $c \bar{c}$ quark pair in its valence. Therefore the $\left(J / \psi \pi^{-}\right)$resonance, otherwise dubbed $Z_{c}(3900)$, has again a minimal valence quark content of four, and $J^{P C}=1^{+-}$.

The $Z_{c}(3900)$ appears in the three states of charge, and the same occurs for $Z_{c}^{\prime}(4020)$, another, slightly heavier $J^{P C}=1^{+-}$resonance, also found in BES III data and also unequivocally exotic. 
The most aged of these resonances is however the neutral X(3872), first observed by Belle in 2003 in the decays of the $B$ meson, and then confirmed by all collider experiments. This can be also be found in the decay product

$$
Y(4260) \rightarrow \gamma X(3872)
$$

as well as promptly produced at the vertex of hadronic collisions. The $X(3872)$ has $J^{P C}=1^{++}$quantum numbers, as confirmed with a high degree of precision. This resonance encodes some very problematic features

1. It does not have charged partners (so far);

2. Its mass is almost perfectly fine-tuned to the mass of the $D^{0} \bar{D}^{0 *}$ meson pair;

3. It decays into $J / \psi \rho$ and $J / \psi \omega$ with almost the same branching fraction;

4. It is an extremely narrow resonance, its width being $\Gamma \lesssim 1 \mathrm{MeV}$;

5. It is almost degenerate to the $J^{P C}=1^{+-} Z_{c}(3900)$ resonance.

The absolute neutralities of the $X(3872)$ and $Y(4260)$, which seems to be experimentally well assessed at the time of this writing, does not speak loud as a signal of a four-quark structure, as it is instead the case for $Z(4430), Z_{c}$ and $Z_{c}^{\prime}$. On the other hand we have to observe that a charmonium cannot decay violating isospin. A more complex quark structure is therefore needed. The spectacular vicinity to the $D^{0} \bar{D}^{0 *}$ threshold has suggested to many that the $X(3872)$ is a $D^{0} \bar{D}^{0 *}$ loosely bound molecule. It is from this latter picture that we will start our discussion on models of $X Y Z$ resonances, in the next Section.

Summarizing, in the charm sector we have $X(3872), Z_{c}(3900), Z_{c}^{\prime}(4020)$ and $Z(4430)$. The latter might not be included among the lowest states - it can be considered as a radial excitation of $Z_{c}(3900)$. In addition we have the $Y(4260)$, which might be considered as an orbital excitation of $X(3872)$.

Therefore, on the basis of some elementary theoretical assumptions, let us stick to the three resonances $X(3872)$, $Z_{c}(3900), Z_{c}^{\prime}(4020)$, reminding that the $X(3872)$ appears only in the neutral state of charge, differently from the other two. Is this pattern repeated in the beauty sector? The answer that we can give to this question, on the basis of present data, is 'not entirely'.

Indeed a pair of resonances have been found in the $b$-sector, $Z_{b}(10610), Z_{b}^{\prime}(10650)$, very close to were expected on the basis of simple quark mass considerations — and again very close to the $B^{(*)} \bar{B}^{*}$ thresholds — and in the three states of charge. But no trace of a neutral or charged $X_{b}$ has been observed so far.

Strange valence quarks can be found in another resonance, first observed in 2009 by the CDF collaboration in

$$
B \rightarrow K(J / \psi \phi)
$$

named $X(4140)$. The quark content is $c \bar{c} s \bar{s}$ and very recently the LHCb collaboration has confirmed its existence and discovered similar resonances at higher masses.

In late 2015, two charged pentaquarks were observed in $\Lambda_{b}$ baryon decays

$$
\Lambda_{b} \rightarrow K^{-}(J / \psi p)
$$

the lowest lying with $J^{P}=3 / 2^{-}$quantum numbers and the next with $J^{P}=5 / 2^{+}$, with masses at $4380 \mathrm{MeV}$ and $4550 \mathrm{MeV}$ respectively. The exotic nature of pentaquarks, sometimes dubbed as $P_{c}$, is very clear, as it is the case for the $Z_{c}$ s and $Z_{b}$ s.

On the basis of what said, we might also observe that low lying four-quark states appear with positive parities whereas five-quark ones with negative. 


\begin{tabular}{lrcll}
\hline \hline State & $M(\mathrm{MeV})$ & $\Gamma(\mathrm{MeV})$ & $\left(I^{G}\right) J^{P C}$ & Details \\
\hline$X(3872)$ & $3871.69 \pm 0.17$ & $<1.2$ & $1^{++}$ & Section 8.1 \\
$Z_{c}(3900)^{+}$ & $3888.4 \pm 1.6$ & $27.9 \pm 2.7$ & $\left(1^{+}\right) 1^{+-}$ & Section 8.2 \\
$Z_{c}^{\prime}(4020)^{+}$ & $4023.9 \pm 2.4$ & $10 \pm 6$ & $\left(1^{+}\right) 1^{+-}$ & Section 8.2 \\
$Y(4260)$ & $4251 \pm 9$ & $120 \pm 12$ & $\left(0^{-}\right) 1^{--}$ & Section 8.4 \\
$Z(4430)^{+}$ & $4478 \pm 17$ & $180 \pm 31$ & $\left(1^{+}\right) 1^{+-}$ & Section 8.5 \\
$X(4140)$ & $4146.5_{-5.3}^{+6.4}$ & $83_{-25}^{+30}$ & $\left(0^{+}\right) 1^{++}$ & Section 8.6 \\
$Z_{b}(10610)^{+}$ & $10607.2 \pm 2.0$ & $18.4 \pm 2.4$ & $\left(1^{+}\right) 1^{+-}$ & Section 8.3 \\
$Z_{b}(10650)^{+}$ & $10652.2 \pm 1.5$ & $11.5 \pm 2.2$ & $\left(1^{+}\right) 1^{+-}$ & Section 8.3 \\
\hline \hline
\end{tabular}

Table 1: A brief summary of the exotic states we will discuss in the following. For more details, see Section 8 , or the subsections devoted to the single particle. Because of isospin breaking, the $I^{G}$ quantum numbers are not defined for the $X(3872)$. With a slight abuse of notation, we will refer to the $C$ eigenvalue for charged states, as to "the $C$ eigenvalue of the neutral isospin partner". For the $Z_{c}(3900)$, we update the PDG 2014 average with the more precise results by BES III.

Table 1 enumerates the properties of the states we briefly discussed here. To our understanding, those listed are the more solid multiquark resonances on experimental grounds. Probably the amount of data available at this time is not yet sufficient to fully understand the nature of $X Y Z P$ resonances, but this is the challenge undertaken by many, which we mean to review in this paper.

\section{Scattering in presence of shallow bound states}

In this and in the following Section 3, we will collect some known results from low energy scattering theory and present, especially in Section 3, some arguments on the limitations of the loosely bound hadron molecule approach to the interpretation of the $X(3872)$ resonance.

The $D^{0} \bar{D}^{0 *}$ molecule interpretation of the $X(3872)$ is however very popular, and is functional to explain the $X$ isospin violation pattern. Thus we start from our discussion on models of multiquark resonances from this interpretation. Some general results rederived here will be of use also in other Sections, when discussing the alternative approaches to the hadron molecule.

\subsection{Shallow bound states: the phase shift from wave mechanics}

Consider two open-charm (or beauty) mesons $a=\left\{D^{0}, B^{0}\right\}$ and $b=\left\{\bar{D}^{* 0}, \bar{B}^{* 0}\right\}$ interacting at low energies through a potential $V$ which is a weak, large distance residual of strong interactions into hadrons. Assume that $V$ allows a discrete level at $-B$ with $B \approx 0$ as in the scheme in Figure 1. What is the effect of this bound state level on the $a b \rightarrow a b$ scattering?

The $a$ and $b$ mesons are approaching to each other from large separation $r$, where we assume they have a total energy $E \approx 0$ and $V(r \rightarrow \infty)=0$ : the system with reduced mass $m=m_{a} m_{b} /\left(m_{a}+m_{b}\right)$ moves to small $r$ values, see Figure 1. The radial wave function $\chi(r)=r R_{\ell=0}(r)$ of the system at large separation is

$$
\chi_{I I}(r)=A \sin \left(k r+\delta_{0}\right)
$$


where the subscript $I I$ defines a region of $r$ such that $r \gg r^{*}, r^{*}$ being a point where the wave functions at short and large separations have to be matched. The region on the left of $r^{*}$ will be named region $I$ and $r^{*}$ is assumed to be quite larger than the effective range $r_{0}$ of $V$ but also quite smaller than $1 / k$. The value of $k$ is $k=\sqrt{2 m E} \approx 0$ and the very long-wavelength in (2.1) allows the approximation

$$
\left.\left.\frac{\chi_{I I}^{\prime}}{\chi_{I I}}\right|_{r^{*}} \simeq \frac{\chi_{I I}^{\prime}}{\chi_{I I}}\right|_{r=0}=k \cot \delta_{0}
$$

The determination of the phase-shift $\delta_{0}$ (which we will calculate in absence of orbital angular momentum, $\ell=0$ ) fully defines the scattering amplitude ${ }^{2}$

$$
f(a b \rightarrow a b)=\frac{e^{2 i \delta_{0}}-1}{2 i k}=\frac{1}{k \cot \delta_{0}-i k}
$$

The logarithmic derivative in (2.2) has to be matched with $\chi_{I}^{\prime} / \chi_{I}$. In region $I$ the Schrödinger equation is

$$
\chi_{I}^{\prime \prime}-2 m V \chi_{I}=0 \quad \text { with } \quad \chi(0)=0
$$

because $V \gg B, E$ all over $I$. Eq. (2.4) means that the ratio $\chi_{I}^{\prime} / \chi_{I}$ will be independent of energy and, in particular, it will stay the same also at some small negative energy $\sim-B$. In that case

$$
\left.\left.\frac{\chi_{I}^{\prime}}{\chi_{I}}\right|_{r^{*}} \simeq \frac{\left(C e^{-\kappa r}\right)^{\prime}}{C e^{-\kappa r}}\right|_{r^{*}}=-\kappa=-\sqrt{2 m B}
$$

where we took $\chi_{I}$ to be the wave function of the stationary state with binding energy $-B$. Therefore realizing the matching condition, i.e. equating (2.2) and (2.5) we get the 'universal' result

$$
\cot \delta_{0}=-\sqrt{\frac{B}{E}}
$$

Both $B$ and $E$ are supposed to be close to zero, but in a fixed, finite, ratio. The phase shift determined does not depend on the details of the unknown potential $V(r)$, but only on its shallow discrete level at $-B$. Any potential which allows a shallow bound state at $-B$ will have the same scattering amplitude at low energies. Since strong interactions between color singlets are expected to be weak and no definite form for their potential exists, it is natural to resort to the results here described, whenever the recoil energies of the color singlets are small enough to allow the approximations needed.

\subsection{Shallow bound states: the phase shift from scattering theory}

The same result (2.6), obtained in wave mechanics as in [12], can be re-derived in scattering theory ${ }^{3}$. The advantage of the following derivation is that it allows to study directly the overlap between a superficial bound state in some potential $V$ and the continuous spectrum scattering state.

The Lippmann-Schwinger equation for the scattering in-state $\Psi_{\alpha}^{+}$is

$$
\Psi_{\alpha}^{+}=\Phi_{\alpha}+\frac{1}{E_{\alpha}-H_{0}+i \epsilon} V \Psi_{\alpha}^{+}
$$

\footnotetext{
${ }^{2}$ The scattering length $a_{s}$ is defined as $\operatorname{limit} \lim _{k \rightarrow 0} k \cot \delta=-1 / a_{s}$ so that the potential scattering cross section, in the same limit, is given by $\sigma=4 \pi a_{s}^{2}$.

${ }^{3}$ We follow closely the derivation in S. Weinberg [13] selecting those key results which are most useful to our discussion.
} 


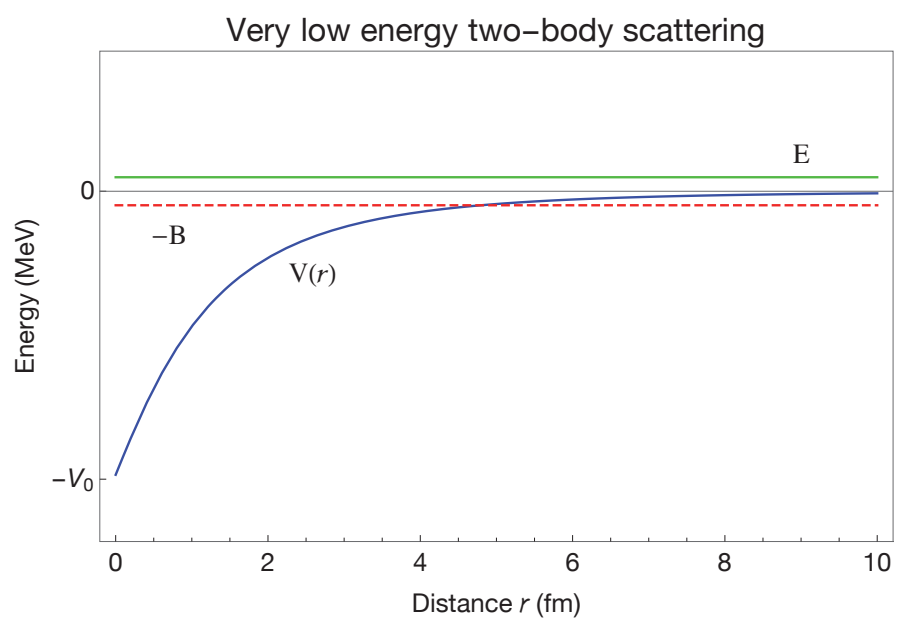

Figure 1: Scheme of the very low energy $(E \approx 0)$ meson-meson scattering in a potential $V$, large-distance residual of strong interactions inside hadrons. Assume $V \gg B, E$ within its $r_{0}$ range, where $-B$ is a discrete level of $V$, assumed to be attractive throughout the entire $r$ range. Here it is assumed that $L=0$ : no centrifugal barrier.

which is the solution of

$$
\left(H_{0}+V\right) \Psi_{\alpha}^{+}=E_{\alpha} \Psi_{\alpha}^{+}
$$

given that the unperturbed free states $\Phi_{\alpha}$ are defined by

$$
\left(E_{\alpha}-H_{0}\right) \Phi_{\alpha}=0
$$

Here $\alpha$ is a compound index labeling types and numbers of particles in the state, momenta and spin 3-components. We consider $V$ in the adiabatic approximation, i.e. $V \rightarrow 0$ for $t \rightarrow-\infty$. This implies that $\Psi_{\alpha}^{+}=\Phi_{\alpha}$ in the far past $t \rightarrow-\infty$, and that the energy eigenvalue $E_{\alpha}$, which is independent of time, is the same for the two states.

Equation (2.7) can be multiplied on the left by $V$

$$
V \Psi_{\alpha}^{+}=V \Phi_{\alpha}+V \frac{1}{E_{\alpha}-H_{0}+i \epsilon} V \Psi_{\alpha}^{+}
$$

and a formal solution of the latter equation is

$$
V \Psi_{\alpha}^{+}=T\left(E_{\alpha}+i \epsilon\right) \Phi_{\alpha}
$$

where

$$
T(W)=V+V \frac{1}{W-H_{0}} T(W)
$$

as is verified by substituting (2.11) into (2.10) and using (2.12) in the resulting lhs of (2.10). The expression in (2.12) can be solved obtaining

$$
T(W)=\left(1-V \frac{1}{W-H_{0}}\right)^{-1} V=V\left(W-H_{0}\right) \frac{1}{W-H}=V+V \frac{1}{W-H} V
$$

where in the last equality one uses $\left(W-H_{0}\right)=(W-H+V)$. The in-state $\Psi_{\alpha}^{+}$, which looks like a free particle state at $t \rightarrow-\infty$, at later times $t \rightarrow+\infty$ looks like ${ }^{4}$

$$
\Psi_{\beta}^{+}=\int_{\alpha} S_{\alpha \beta} \Phi_{\alpha}
$$

${ }^{4}$ given that out-states $\Psi_{\alpha}^{-}$look like $\Phi_{\alpha}$ at late times 
where the $S$-matrix is found to be ${ }^{5}$

$$
S_{\alpha \beta}=\delta(\alpha-\beta)-2 \pi i \delta\left(E_{\beta}-E_{\alpha}\right) T_{\alpha \beta}
$$

and

according to (2.11).

$$
T_{\alpha \beta} \equiv\left\langle\Phi_{\alpha} \mid T \Phi_{\beta}\right\rangle=\left\langle\Phi_{\alpha} \mid V \Psi_{\beta}^{+}\right\rangle
$$

The second term on the rhs of Eq. (2.13) allows two terms by completeness: one can be obtained summing on bound states $\psi_{i}$, if any, the other summing on in-states $\Psi_{\gamma}^{+}$. Thus we get the Low equation,

$$
T_{\alpha \beta}\left(E_{\alpha}+i \epsilon\right)=V_{\alpha \beta}+\oint_{i} \frac{\left\langle\Phi_{\beta} \mid V \psi_{i}\right\rangle\left\langle\Phi_{\alpha} \mid V \psi_{i}\right\rangle^{*}}{E_{\alpha}-E_{i}}+\int_{\gamma} \frac{T_{\alpha \gamma} T_{\beta \gamma}^{*}}{E_{\alpha}-E_{\gamma}+i \epsilon}
$$

where we sum over both the discrete and continuum labels (like momentum) characterizing the bound states $\psi_{i}$ and we defined $V_{\alpha \beta}=\left\langle\Phi_{\alpha} \mid V \Phi_{\beta}\right\rangle$. The completeness relation over the states in the discrete and continuous spectra requires a contribution to the $T$ matrix also from bound states: the form $\Psi_{\alpha}^{+}$will assume at later times $t \rightarrow+\infty$ (scattering) is affected by bound states in the potential $V$. We showed above that even not knowing the details of $V(r)$, the scattering phase shift can be determined if it is assumed that the potential admits a shallow bound state at $E_{1}=-B$ and scattering occurs at very low energy $E$. Following the derivation in [13] we will re-obtain the same result (2.6) for the phase shift by solving the Low equation (2.17), i.e. determining $T_{\alpha \beta}$ from the equation above, under the same general assumptions on $V$ and $E$.

At very low energy $E_{\alpha}$, and in presence of a single shallow bound state at $-B$, very close to the onset of the continuum, the second term on the rhs of (2.17) will be dominated by ${ }^{6}$

$$
\frac{\left\langle\Phi_{\beta} \mid V \psi_{i}\right\rangle\left\langle\Phi_{\alpha} \mid V \psi_{i}\right\rangle^{*}}{E_{\alpha}+B}
$$

with $E_{\alpha} \approx B \approx 0$. This also dominates over transitions in the continuum spectrum $V_{\alpha \beta}$, thus we will neglect the latter. This term is particularly relevant to our discussion and will be recalled in Section 6.

By $\Phi_{\alpha}$ we mean a two-particle state with some total energy $E=E_{\alpha}$ relative to the rest frame and zero total momentum $\boldsymbol{P}$. In this discussion we will only consider $S$-wave states. To simplify the notation, we will not explicit spin degrees of freedom. Therefore $\Phi_{\alpha} \equiv \Phi_{E, \boldsymbol{P}=0}$ in the center of mass of the two-particle system. These states will have the convenient normalization

$$
\left\langle\Phi_{E^{\prime}, \boldsymbol{P}^{\prime}} \mid \Phi_{E, \boldsymbol{P}}\right\rangle=\delta\left(E^{\prime}-E\right) \delta\left(\boldsymbol{P}^{\prime}-\boldsymbol{P}\right)
$$

if

$$
\Phi_{E, \boldsymbol{P}}=\int d^{3} p \frac{1}{\sqrt{\mu|\boldsymbol{p}|}} \delta\left(E-E_{1}-E_{2}\right) \Phi_{\boldsymbol{p} ; \boldsymbol{P}-\boldsymbol{p}}
$$

where the latter two-particle states $\Phi_{\boldsymbol{p} ; \boldsymbol{P}-\boldsymbol{p}}$ have conventional continuous spectrum normalization

$$
\left\langle\Phi_{\boldsymbol{p}_{1}^{\prime} ; \boldsymbol{p}_{2}^{\prime}} \mid \Phi_{\boldsymbol{p}_{1} ; \boldsymbol{p}_{2}}\right\rangle=\delta\left(\boldsymbol{p}_{1}^{\prime}-\boldsymbol{p}_{1}\right) \delta\left(\boldsymbol{p}_{2}^{\prime}-\boldsymbol{p}_{2}\right)
$$

Here $\mu=E_{1} E_{2} /\left(E_{1}+E_{2}\right)$ derives from the definition of relative velocity in the center of mass

$$
v=\frac{|\boldsymbol{p}|}{E_{1}}+\frac{|\boldsymbol{p}|}{E_{2}}
$$

\footnotetext{
${ }^{5}$ Substitute (2.15) and (2.16) into (2.14) finding $\Psi_{\beta}^{+}=\Phi_{\beta}-2 \pi i \int_{\alpha} \delta\left(E_{\beta}-E_{\alpha}\right)\left\langle\Phi_{\alpha} \mid V \Psi_{\beta}^{+}\right\rangle \Phi_{\alpha}$. This is the same result that would be obtained by $\left.i\right)$ integrating (in $\beta$ ) the Lippmann-Schwinger equation (2.7) with weights $f(\beta) e^{-i E_{\beta} t}$, where $f$ is a smooth function $i i$ ) using completeness on $\Phi_{\alpha}$ in the second term on the rhs of (2.7) iii) taking the $t \rightarrow+\infty$ limit, i.e. computing the contour integral on the $E_{\beta}$ lower half plane, keeping $\int_{\beta} \delta\left(E_{\beta}-E_{\alpha}\right) \cdots$ to enforce the result of the residue theorem. The factor $e^{-i E_{\beta} t}$ is needed to perform the contour integral.

${ }^{6}$ For the sake of comparison with (6.4) we have the interesting case $\alpha=\beta$ in which $T_{\alpha \alpha}=\frac{\left|\left\langle\psi_{i} \mid V \Phi_{\alpha}\right\rangle\right|^{2}}{E_{\alpha}-E_{i}+i \epsilon}$.
} 
and in general $E_{1}=\sqrt{m_{1}^{2}+\boldsymbol{p}^{2}}, E_{2}=\sqrt{m_{2}^{2}+(\boldsymbol{P}-\boldsymbol{p})^{2}}(\boldsymbol{P}=0$ in the center of mass $)$. In the non-relativistic limit $\mu \equiv m$, the reduced mass ${ }^{7}$.

The numerator in (2.18), because of the $1 / \sqrt{|\boldsymbol{p}|}$ factor in (2.20), would be singular for very low recoils $|\boldsymbol{p}| \rightarrow 0$, which contradicts the analyticity requirement on the $S$-matrix. On the other hand

$$
\left\langle\Phi_{p ;-p} \mid V \psi_{P}\right\rangle
$$

has to be an analytic function when $|\boldsymbol{p}| \rightarrow 0$ - roughly speaking a constant at $|\boldsymbol{p}|=0$. Here the bound state is defined by the total momentum $\boldsymbol{P}$ which in (2.24) is set to be zero (see (2.26)). Therefore using (2.20) in the determination of

$$
\left\langle\Phi_{\beta} \mid V \psi\right\rangle \equiv\left\langle\Phi_{E^{\prime}, 0} \mid V \psi_{P}\right\rangle
$$

we find

$$
\left\langle\Phi_{E^{\prime}, 0} \mid V \psi_{\boldsymbol{P}}\right\rangle \sim \delta(\boldsymbol{P}) \int \sqrt{p} E_{1} d E_{1} \delta\left(E^{\prime}-E_{1}-E_{2}\right)=g \delta(\boldsymbol{P}) \sqrt{\left|\boldsymbol{p}\left(E^{\prime}\right)\right|}
$$

where $p=\sqrt{2 \mu E}$ and $\mu$ is the reduced mass ${ }^{8}$. Here the constant $g$ measures the overlap of the bound state $\psi_{P=0}$ to the continuum one $\Phi_{E^{\prime}, \mathbf{0}}$. The constant $g$ can be determined as a function of $\mu$ and $B$ by substituting $V=H-H_{0}$ in (2.26). We report this solution below.

The bound state term (2.18) can therefore be rewritten as

$$
\frac{\sqrt{p(E) p\left(E^{\prime}\right)}}{E+B}|g|^{2} \delta(\boldsymbol{P}) \delta\left(\boldsymbol{P}^{\prime}\right)
$$

The denominator is the energy gap between $E$ and $-B$ as in Figure 1. The smaller this energy gap, the better is the approximation of neglecting $V_{\alpha \beta}$ in the Low equation, which otherwise would require the knowledge of $V$. Since we have in mind the residual strong interactions between color neutral hadrons, and have little clue on the explicit form of $V$, it is better to be in the approximation of small $E+B$ gap.

As for the $T$-matrix elements in the Low equation (2.17) they are expressed in the center-of-mass of the two-particle system as

$$
T_{\alpha \beta}=T_{E, \mathbf{0} ; E^{\prime}, \boldsymbol{P}^{\prime}} \equiv T\left(E, E^{\prime}\right) \delta(\boldsymbol{P})
$$

so that (2.17) becomes

$$
T\left(E, E^{\prime}\right)=\frac{\sqrt{p(E) p\left(E^{\prime}\right)}}{E+B}|g|^{2}+\int_{0}^{\infty} d E^{\prime \prime} \frac{T\left(E, E^{\prime \prime}\right) T^{*}\left(E^{\prime}, E^{\prime \prime}\right)}{E-E^{\prime \prime}+i \epsilon}
$$

This version of the Low equation admits a solution

$$
\begin{aligned}
T\left(E, E^{\prime}\right) & =\sqrt{p(E) p\left(E^{\prime}\right)} t(E) \\
t(E) & =\frac{|g|^{2}}{E+B}+\int_{0}^{\infty} d E^{\prime} \frac{p\left(E^{\prime}\right)}{E-E^{\prime}+i \epsilon}\left|t\left(E^{\prime}\right)\right|^{2}
\end{aligned}
$$

\footnotetext{
${ }^{7}$ Indeed, using (2.21) we find that

$$
\begin{aligned}
\left\langle\Phi_{E^{\prime}, \boldsymbol{P}^{\prime}} \mid \Phi_{E, \mathbf{0}}\right\rangle & =\int d^{3} p \frac{1}{\sqrt{\mu|\boldsymbol{p}|}} \delta\left(E^{\prime}-E_{1}-E_{2}\right) \int d^{3} p^{\prime} \frac{1}{\sqrt{\mu\left|\boldsymbol{p}^{\prime}\right|}} \delta\left(E-E_{1}^{\prime}-E_{2}^{\prime}\right)\left\langle\Phi_{\boldsymbol{p} ; \boldsymbol{P}^{\prime}-\boldsymbol{p}} \mid \Phi_{\boldsymbol{p}^{\prime} ;-\boldsymbol{p}^{\prime}}\right\rangle= \\
& =\int d^{3} p \frac{1}{\mu|\boldsymbol{p}|} \delta\left(E^{\prime}-E_{1}-E_{2}\right) \delta\left(E-E_{1}-E_{2}\right) \delta\left(\boldsymbol{P}^{\prime}\right)= \\
& \equiv \frac{\delta\left(\boldsymbol{P}^{\prime}\right)}{\mu} \int p d p \delta\left(E^{\prime}-E\right) \delta\left(E-E_{1}-E_{2}\right)=\frac{\delta\left(\boldsymbol{P}^{\prime}\right)}{\mu} \delta\left(E^{\prime}-E\right) \int E_{1} d E_{1} \delta\left(E-E_{1}-E_{2}\right)
\end{aligned}
$$

and the last integral gives exactly $\mu$ as can bee seen by replacing $E_{2}=\sqrt{E_{1}^{2}-m_{1}^{2}+m_{2}^{2}}$

${ }^{8} \mathrm{We}$ have also used the last integral in (2.23) and $\boldsymbol{p}^{2} / 2 m_{1}+\boldsymbol{p}^{2} / 2 m_{2}=E$
} 
as can be seen by direct substitution in (2.29). The function $t(E)$ can be expressed in terms of $p(E)=\sqrt{2 \mu E}$ as follows

$$
t(E)=\left(\frac{E+B}{|g|^{2}}+(E+B)^{2} \int_{0}^{\infty} d E^{\prime} \frac{\sqrt{2 \mu E^{\prime}}}{\left(E^{\prime}+B\right)^{2}\left(E^{\prime}-E-i \epsilon\right)}\right)^{-1}
$$

The derivation of the previous solution is based on dispersion theory integrals and can be found in [13]. As explained there, it is not unique as it relies on some analyticity requirements. However, all the different solutions converge to Eq. (2.31) in the $B \rightarrow 0$ limit.

The latter integral can be computed in the complex plane with a cut on the real positive axis and using the residue theorem at the poles $E^{\prime}=-B$ and $E^{\prime}=E+i \epsilon$. This calculation gives

$$
t(E)=\left(\frac{E+B}{|g|^{2}}+\frac{\pi(B-E)}{2} \sqrt{\frac{2 \mu}{B}}+i \pi \sqrt{2 \mu E}\right)^{-1}
$$

As a last step towards the solution of the Low equation, let us analyze the coupling $g$ reconsidering Eq. (2.26)

$$
g \delta(\boldsymbol{P}) \sqrt{|\boldsymbol{p}(E)|}=\left\langle\Phi_{E, \mathbf{0}} \mid V \psi_{\boldsymbol{P}}\right\rangle=\left\langle\Phi_{E, \mathbf{0}} \mid\left(H-H_{0}\right) \psi_{\boldsymbol{P}}\right\rangle=(-B-E)\left\langle\Phi_{E, \mathbf{0}} \mid \psi_{\boldsymbol{P}}\right\rangle
$$

thus

On the other hand

$$
\left\langle\Phi_{E, \mathbf{0}} \mid \psi_{\boldsymbol{P}}\right\rangle=-g \delta(\boldsymbol{P}) \frac{\sqrt{|\boldsymbol{p}(E)|}}{E+B}
$$

$$
\delta(\boldsymbol{P})=\left\langle\psi_{\boldsymbol{P}} \mid \psi_{\mathbf{0}}\right\rangle=\int_{E, \boldsymbol{Q}}\left\langle\psi_{\boldsymbol{P}} \mid \Phi_{E, \boldsymbol{Q}}\right\rangle\left\langle\Phi_{E, \boldsymbol{Q}} \mid \psi_{\mathbf{0}}\right\rangle=|g|^{2} \delta(\boldsymbol{P}) \int_{E}\left(\frac{\sqrt{|\boldsymbol{p}(E)|}}{E+B}\right)^{2}
$$

or

$$
1=|g|^{2} \int_{0}^{\infty} d E\left(\frac{\sqrt{|\boldsymbol{p}(E)|}}{E+B}\right)^{2}
$$

with the solution ${ }^{9}$

$$
|g|^{2}=\sqrt{\frac{2 B}{\mu \pi^{2}}}
$$

which gives for the elastic $T$-matrix

$$
T(E, E)=\frac{1}{\pi} \frac{\sqrt{E}}{\sqrt{B}+i \sqrt{E}}
$$

We observe here the $B \sim|g|^{4}$ dependency which will be reobtained later, in a similar form (3.10), when connecting the binding energy of the $X(3872)$, interpreted as as a loosely bound molecule of open charm mesons, and the effective strong coupling regulating its decay in the constituent mesons. $M$

The phase shifts for elastic scattering, in the center of mass, are defined by the equation for the scattering amplitude

$$
M_{E, 0 ; E, 0}=e^{2 i \delta(E)}-1=-2 \pi i T(E, E)
$$

where

$$
S_{\alpha \beta}=\delta(\alpha-\beta)+\delta\left(E_{\alpha}-E_{\beta}\right) \delta\left(\boldsymbol{P}_{\alpha}-\boldsymbol{P}_{\beta}\right) M_{\alpha \beta}
$$

to be compared with the expression in (2.15).

\footnotetext{
${ }^{9}$ More comments on this formula can be found in [14].
} 
Combining (2.38) and (2.39) gives again (2.6)

$$
\cot \delta=-\sqrt{\frac{B}{E}}
$$

where $B, E \rightarrow 0$ remaining in a fixed ratio.

We use this result on the phase shifts in the next Section. We notice again that the formula obtained does not depend on the details of the potential, but only on the position of the bound state energy level $B$ compared to the energy $E$ of the scattering system. This is particularly useful in the case of strong interactions for which will not need to model the static potential between two color-neutral hadrons. We might simply assume that there is some residual strong interaction at long distances between two hadrons, like the $D^{0}$ and $D^{* 0}$ mesons in our specific case, and that the potential is deep enough to accommodate a superficial (close to continuum) discrete level at energy $-B$.

From Eq. (2.37) we see that the overlap between the bound state $\psi_{P=0}$ and the the continuum state $\Phi_{E^{\prime}, 0}$ goes to zero as $B \rightarrow 0$.

\section{Loosely bound molecules}

This section is devoted to the molecular picture of the $X(3872)$, trying to cover most of its implications. We will also discuss how the relation between binding energy and total decay rate constrains the most simple hadron molecule interpretation, with particular reference to the case of the $X(3872)$, and we will remark that a deuteron-like interpretation of $X$ has to confront with data on the production of light nuclei in in $p p$ and $\mathrm{Pb}-\mathrm{Pb}$ collisions at the LHC.

\subsection{Relation between binding energy and the decay rate of $X(3872)$}

We can use the result (2.41) in (2.3), or equivalently divide the $r h s$ of (2.39) by $2 i k$, to obtain

$$
f(a b \rightarrow a b)=\frac{1}{\sqrt{2 m E}} \frac{-\sqrt{E}}{\sqrt{B}+i \sqrt{E}}=-\frac{1}{\sqrt{2 m}} \frac{\sqrt{B}-i \sqrt{E}}{E+B}
$$

where $\mu=m$, the usual reduced mass. The scattering amplitude displays a pole at $E=-B$, and the cross section $4 \pi|f|^{2}$ has a resonant character

$$
\sigma=\frac{2 \pi}{m} \frac{1}{E+B}
$$

at $E \sim B \approx 0$. Within a interval of time $\tau \sim 1 /(E+B)$ the free particle can be 'locked' in the finite motion region $I$ in Figure 1 and behave temporarily as a bound state $c$. The value of $\sigma$ close to the resonance, is larger than $4 \pi r_{0}^{2}$ (the 'natural' size for the cross section), where $r_{0}$ is the range of the potential, because $k r_{0} \ll 1$ meaning $r_{0}^{2} \ll 1 / E \sim \sigma$ at resonance. We limit our considerations to the $S$-wave cross section because we have in mind the problem of the $X(3872)$ as a resonance in the $D D^{*}$ scattering at low energies $k \rightarrow 0$ where $\delta_{\ell} \sim k^{2 \ell+1}$. Also, since $X$ has $1^{++}$quantum numbers whereas $D, D^{*}$ are $0^{-}$and $1^{-}$respectively a $P$-wave scattering is forbidden by parity, while the $D$-wave option would give a very small phase shift.

Here comes indeed the main point: in quantum mechanics the formation of a resonance requires the interplay between the attractive potential and a repulsive centrifugal barrier, especially if the potential is weak, as in the case of strong interactions between color singlets. This does not happens for an $S$-wave scattering, and hence it does not happen for the $X(3872)$. 
In presence of a centrifugal barrier, a 'quasi-discrete' (metastable state) positive energy level at $\epsilon>0$ would be possible, allowing a narrow resonance: the energy region in which $f_{\ell} \sim 1 / k$, i.e. large compared with $r_{0}$, has a relative width $\Delta E / \epsilon \sim k^{2 \ell-1}$ for $\ell \neq 0{ }^{10}$.

Stated differently, even though the cross section enhancement (3.2) might be possible (although $E \sim B$ is a rare event in hadron collisions with hard cuts, see Appendix A), it cannot anyway generate narrow resonances which would require higher partial waves. In the case of the $X(3872)$ at least $\ell=2$ would be required: but such partial waves do not give significant contributions to the phase shifts when $k \rightarrow 0$. A loosely bound $D D^{*}$ hadron molecule in a high orbital angular momentum state would tend to be even larger than what it is in $S$-wave, damping the $J / \psi(\rho / \omega)$ decay rates.

In quantum field theory, the process is $a b \rightarrow c \rightarrow a b$ where we associate the propagator function to the temporary bound state $c$. In this case, in place of the potential scattering description reported above, the interaction between $a$ and $b$ is determined by the coupling of the two components to $c$. The propagator pole dominates the scattering amplitude $M$ in $^{11}$

$$
f(\beta \rightarrow \alpha)=-\frac{1}{8 \pi \mathcal{E}} M_{\alpha \beta}
$$

which in the standard relativistic formalism is defined by

$$
S_{\alpha \beta}=\delta(\alpha-\beta)-i(2 \pi)^{4} \delta^{4}\left(p_{\alpha}-p_{\beta}\right) M_{\alpha \beta}
$$

Eq. (3.3) is for elastic two-to-two body scattering, computed with a relativistic formalism (and including the phase space calculation of the final particles). Therefore we can write

$$
f(a b \rightarrow c \rightarrow a b)=\frac{1}{8 \pi\left(m_{a}+m_{b}+E\right)} G^{2} \frac{1}{\left(p_{a}+p_{b}\right)^{2}-m_{c}^{2}}
$$

where $E$ has the same meaning given in the previous section: total energy relative to the rest mass. Since $E \ll m_{a, b}$ we will neglect it in the first factor on the $l h s$. The constant $G$ here defines the strong coupling of the free components $a b$ to the bound state $c, G \equiv G_{a b c}$.

Recall now that

$$
\begin{aligned}
m_{c} & =m_{a}+m_{b}-B \\
\left(p_{a}+p_{b}\right) & \simeq m_{a}+m_{b}+E
\end{aligned}
$$

so that

$$
\left(p_{a}+p_{b}\right)^{2}-m_{c}^{2} \simeq 2\left(m_{a}+m_{b}\right)(E+B)
$$

where the very small terms $B^{2}$ and $E^{2}$ have been neglected. Therefore we have

$$
f(a b \rightarrow c \rightarrow a b) \simeq \frac{G^{2}}{16 \pi\left(m_{a}+m_{b}\right)^{2}} \frac{1}{E+B}
$$

Expressions (3.1) and (3.9) are two different descriptions of the same process whose cross section is obtained by $d \sigma / d \Omega=|f|^{2}$. The first one is derived in low energy scattering theory. The second involves the propagation of a

\footnotetext{
${ }^{10}$ At low energies $f_{\ell} \approx \delta_{\ell} / k \sim k^{2 \ell}$. Thus, if we write $f_{\ell}=1 /\left(g_{\ell}-i k\right)$, the $g_{\ell}$ expansion starts at $k^{-2 \ell}\left(k^{-2 \ell} \gg k\right)$, larger terms like $k^{-2 \ell-1} \ldots$ are neglected. Writing $g_{\ell}$ as $g_{\ell} \sim B(E-\epsilon)+\cdots$, with $B \sim k^{-2 \ell}$, we are retaining the first two terms, $k^{-2 \ell}$ and the subdominant $k^{-2 \ell+1}$. Interpret $\epsilon>0$ as the quasi-discrete energy level quoted above. In order to have $f_{\ell} \sim 1 / k$, i.e. large compared with $r_{0}$, we need $E \sim \epsilon$. This region has a width $\Delta E \sim k^{2 \ell+1}$, and the relative width is $\Delta E / \epsilon=\Delta E / k^{2} \sim k^{2 \ell-1}$. We finally observe that the low energy behavior tan $\delta_{\ell} \approx \delta_{\ell} \sim k^{2 \ell+1}$ does not hold for $B=0$ in the case of (2.6) where $\tan \delta_{0} \rightarrow \infty$.

${ }^{11}$ dividing $M_{\alpha \beta}$ by $(2 \pi)^{3}$ and including the wave function normalizations $1 / \sqrt{2 \mathcal{E}}$ in Eq. (3.6.9) in Ref. [15]. Here $\mathcal{E}$ is the total energy which in the non-relativistic approximation is $\mathcal{E}=E+m_{a}+m_{b}$.
} 
virtual state as in quantum field theory. The non-physical pole appears in both with the same power. Indeed, at the $E=-B$ pole we find a relation between binding energy and strong coupling $G$ which can be written as ${ }^{12}$

$$
B \simeq \frac{G^{4}}{512 \pi^{2}} \frac{m^{5}}{\left(m_{a} m_{b}\right)^{4}}
$$

The advantage of this expression is that the strong coupling constant $G$ is the same as that entering in the computation of the decay rate $c \rightarrow a b$, which in general is an accessible experimental information. Observe that the scaling relation between $B$ and $G$

$$
B \sim G^{4}
$$

was already appreciated in the discussion of the Low equation (see (2.37)), but in that case $g$ is normalized with the nonrelativistic convention: $G=4 \pi\left(m_{a}+m_{b}\right) g$. Notice that in the calculation of the rate $\Gamma(c \rightarrow a b)=G^{2} \Phi / 2\left(m_{a}+m_{b}\right)$, $G$ has dimensions of energy, the two body phase space $\Phi$ being dimensionless.

We might suppose that the experimentally observed $X(3872)$ resonance is the effect of a shallow bound state in the potential $V$, describing the strong interaction within the $\bar{D}^{0} D^{* 0}$ meson pair when low energy scattering $\bar{D}^{0} D^{* 0} \rightarrow$ $\bar{D}^{0} D^{* 0}$ occurs. As commented above, the $\bar{D}^{0} D^{* 0}$ system might temporarily behave as a bound state, with very loose binding energy $B \approx 0$. Indeed we know that $B=m_{D}+m_{D^{*}}-m_{X} \approx 0$. We found that a relation between $B$ and the strong coupling describing the decay of $X$ into its components $\bar{D}^{0} D^{* 0}$ holds.

In the case at hand $m_{a}=m_{D}, m_{b}=m_{D^{*}}$ and we define the strong coupling $G$ through ${ }^{13}$

$$
\left\langle D^{0} \bar{D}^{0 *}(\epsilon, q) \mid X(\lambda, P)\right\rangle=G \lambda \cdot \epsilon^{*}
$$

Taking into account the spin of $D^{*}$ and $X$, one should rather use

$$
G^{2} \rightarrow G^{2} \frac{1}{3}\left(2+\frac{\left(m_{X}^{2}+m_{D^{*}}^{2}-m_{D}^{2}\right)^{2}}{4 m_{X}^{2} m_{D^{*}}^{2}}\right)
$$

which, however, turns out to be numerically $\simeq G^{2}$. The actual value of $G$ is extracted from data on the branching ratio $\mathcal{B}(X \rightarrow \bar{D} D \pi)$, which is measured experimentally to be larger than $32 \%$. However the total width is poorly known: $\Gamma(X) \lesssim 1.2 \mathrm{MeV}$. Using these two extreme values and the $X \rightarrow \bar{D} D \pi$ decay rate

$$
\begin{aligned}
\Gamma(X \rightarrow \bar{D} D \pi) & =\int_{\left(m_{D}+m_{\pi}\right)^{2}}^{\left(m_{X}-m_{D}\right)^{2}} d s \frac{1}{3} \frac{1}{8 \pi m_{X}^{2}} 3(G \sqrt{2})^{2} p^{*}\left(m_{X}^{2}, m_{D}^{2}, s\right) \times \\
& \times \frac{1}{\pi} \frac{s / m_{D^{*}} \Gamma_{D^{*}} \mathcal{B}\left(D^{*} \rightarrow D \pi\right)}{\left(s-m_{D^{*}}^{2}\right)^{2}+\left(s / m_{D^{*}} \Gamma_{D^{*}}\right)^{2}} \frac{m_{D^{*}}}{\sqrt{s}} \frac{p^{*}\left(s, m_{D}^{2}, m_{\pi}^{2}\right)}{p^{*}\left(m_{D^{*}}^{2}, m_{D}^{2}, m_{\pi}^{2}\right)}
\end{aligned}
$$

where the decay momentum is $p^{*}(x, y, z)=\sqrt{\lambda(x, y, z)} / 2 \sqrt{x}, \lambda$ being the Källén triangular function, it is found that $G \approx 4 \mathrm{GeV}$. Considering for example a branching fraction of $\mathcal{B}(X \rightarrow \bar{D} D \pi) \simeq 0.32$, we obtain $B=B_{\exp }$ on assuming a total width of the $X$ as large as $\approx 300 \mathrm{keV}$ : lower values of $\Gamma_{X}$ would also be possible for higher branching ratios $\mathcal{B}(X \rightarrow \bar{D} D \pi)$, whereas higher $\Gamma_{X}$ values are excluded. These conclusions are displayed by the shaded areas in Figure 2, which are limited by the hyperbolae

$$
\mathcal{B}(X \rightarrow \bar{D} D \pi) \cdot \Gamma(X) \sim G^{2} \sim \sqrt{B}
$$

obtained fixing the value of $B$ [17]. The not-excluded region is quite small and a high resolution on the width of the $X(3872)$ would be necessary — one should be able to go below $\Gamma(X) \lesssim 300 \mathrm{keV}$. An accurate determination of $\mathcal{B}(X \rightarrow \bar{D} D \pi)$ and $\Gamma(X)$ would allow to test (3.10) and the molecular interpretation behind it.

\footnotetext{
${ }^{12} \mathrm{~A}$ derivation of this formula, which is a straightforward consequence of the considerations in [12] leading to (3.1), can be found in [16].

${ }^{13}$ Since $X$ has positive charge conjugation, the final state is $|f\rangle=\left(\left|D^{0} \bar{D}^{0 *}\right\rangle-\left|\bar{D}^{0} D^{0 *}\right\rangle\right) / \sqrt{2}$. When extracting $g$ defined in (3.12) from data a factor of $\sqrt{2}$ has to be included: $G \rightarrow \sqrt{2} G$.
} 


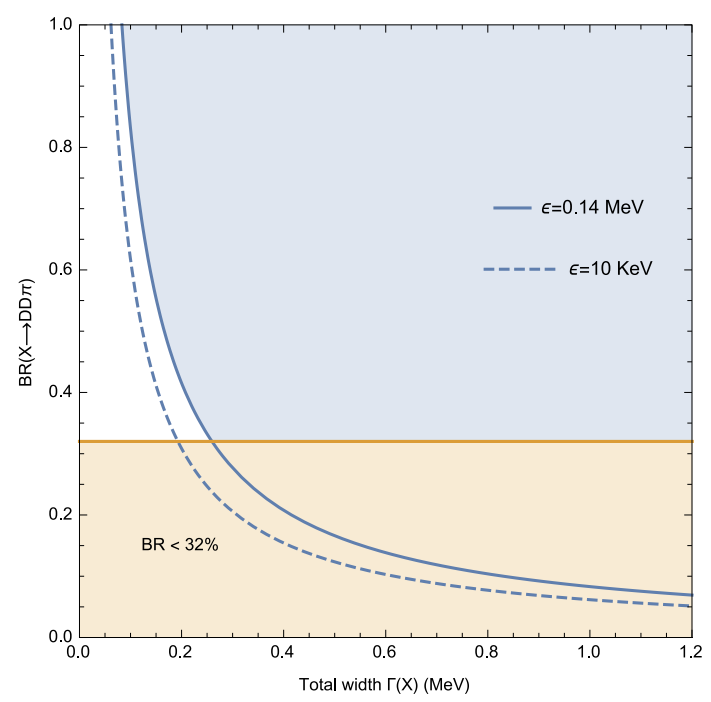

Figure 2: Given the experimentally excluded region (shaded), a loosely bound molecule, has total width $\Gamma_{X}$, branching ratio $\mathcal{B}(X \rightarrow \bar{D} D \pi)$ and binding energy $B=m_{D}+m_{D^{*}}-m_{X}$ as in the plot. The approximate values are used: $m_{X}=3871.66 \mathrm{MeV}, m_{D}=1864.84 \mathrm{MeV}$ and $m_{D^{*}}=2006.96 \mathrm{MeV}$ for $B=0.14 \mathrm{MeV}$ and a slightly modified value for $m_{X}=3871.79 \mathrm{MeV}$ in order to get $B=10 \mathrm{keV}$. A measurement of the total width $\Gamma(X)$ ending in the shaded area would falsify the low energy resonance scattering mechanism described.

\subsection{Scattering length of the $X(3872)$ as a loosely bound molecule}

The extremely small binding energy of the $X(3872)$ makes it an ideal candidate for a loosely bound molecule, and a perfect example where the rigorous low energy universality could be applied. This was firstly done in [18] and slightly later in [19] to study different implications of the shallow bound state theory to the study of the $X$.

A priori, the quantum mechanical state for the $X(3872)$ can be a superposition of all the hadronic states with the right quantum numbers, $J^{P C}=1^{++}[19]$

$$
|X\rangle=\sqrt{Z_{\mathrm{mol}}} \int \frac{d^{3} p}{(2 \pi)^{3}} \widetilde{\psi}(p) \frac{\left|D^{0}(\boldsymbol{p}) \bar{D}^{* 0}(-\boldsymbol{p})\right\rangle+\left|\bar{D}^{0}(\boldsymbol{p}) D^{* 0}(-\boldsymbol{p})\right\rangle}{\sqrt{2}}+\sum_{H} \sqrt{Z_{H}}|H\rangle
$$

In the previous expression $\widetilde{\psi}(p)$ is the momentum space wave function of the $D$ mesons - see Eq. (3.19) below — while $|H\rangle$ are other hadronic states, discrete or continuous. These can be other molecular components like $\left|D^{+}(\boldsymbol{p}) D^{*-}(-\boldsymbol{p})\right\rangle$, charmonium states or even compact four-quark objects. The presence of the latter ones will play an essential role in the mechanism explained in Section 6. The key question is which of these different components is relevant to the total wave function or, in other words, how the probabilities $Z_{i}$ look like.

The probabilities for the states scale as $Z_{H} \sim 1 / \delta_{H}^{2} a_{\mathrm{s}}$, where $\delta_{H}$ is the mass splitting of the $X$ - or of the loosely bound molecule in general — with respect to the state $H .{ }^{14}$ This behavior has been computed in [19] using an explicit effective field theory calculation but can also be seen qualitatively from the more general Eq. (2.34). It tells us that

$$
\sqrt{Z_{H}} \sim \frac{g}{\delta_{H}} \Rightarrow Z_{H} \sim \frac{|g|^{2}}{\delta_{H}^{2}} \sim \frac{\sqrt{B}}{\delta_{H}^{2}} \sim \frac{1}{\delta_{H}^{2} a_{\mathrm{s}}}
$$

\footnotetext{
${ }^{14}$ The fact that $\delta_{H} \simeq 0$ and $8 \mathrm{MeV}$ for $D^{0} \bar{D}^{* 0}$ and $D^{+} D^{*-}$ respectively implies a suppression of the second one in Eq. (3.16). This explains the observed isospin violation of the $X$ from a molecular point of view.
} 
where we used $E+B \approx \delta_{H}$ and that fact that, as can be seen from (2.6) and (2.41), in the $E \sim B \sim 0$ limit the scattering length of the $a b \rightarrow a b$ process becomes

$$
a_{\mathrm{s}}=\frac{1}{\sqrt{2 m B}}
$$

which is divergent in the strict $B \rightarrow 0$ limit. Applying this result to the case of the $X$ with $m=m_{D} m_{D^{*}} /\left(m_{D}+m_{D^{*}}\right)$ and $B=m_{D}+m_{D^{*}}-m_{X}$ one finds $a_{\mathrm{s}} \simeq 11.97 \mathrm{fm}$, an extremely large number. It has been shown in [20] that this scattering length can hardly be reconciled with the one extrapolated from experimental data on the full width of $X(3872)$, since the latter one appears to be at least a factor of 3-4 times smaller that the one expected from shallow states theory.

Moreover, the wave function of the loosely bound molecule for separations $r \gg \ell, \ell$ being the typical size of the components, is universally given by $[12,21]$ (see also Eq. (2.5))

$$
\chi(r) \propto e^{-r / a_{\mathrm{s}}} \Rightarrow R_{\ell=0}(r)=\frac{\chi(r)}{r} \propto \frac{e^{-r / a_{\mathrm{s}}}}{r}
$$

Therefore, the typical size of the loosely bound molecule is $a_{\mathrm{s}}$ itself. The $X(3872)$ would therefore be an unnaturally extended objected, much larger than the typical hadrons (and $~ 3$ times larger than the deuteron) and of the typical range of interaction between heavy mesons, $1 / m_{\pi}$. In other words, while the binding energy for the $X$ would be expected to be of order $m_{\pi}^{2} / 2 m \simeq 10 \mathrm{MeV}$, while the actual value $B \simeq 0.14 \mathrm{MeV}$ is much smaller. From a certain point of view this feature can explain some of the peculiar properties of this resonance but, on the other hand, it requires a good degree of fine tuning that, if not properly explained, might make the molecular interpretation questionable.

In [19] two possible explanations for this phenomenon are given. The first possibility is to have a fine tuning that only interests the $D^{0} \bar{D}^{* 0}$ component of (3.16), without influencing the others. This could happen, for example, in the meson-meson potential so that it allows for a bound state very close to threshold. If the other detunings $\delta_{H}$ are larger than the natural energy scale, the very large scattering length would suppress all the other states $|H\rangle$. In this case, the $X(3872)$ would be a purely $D^{0} \bar{D}^{* 0}$ molecular state. Although this is an appealing solution, we will see in the next section that a real, negative energy meson molecule faces severe difficulties when the prompt production at hadron colliders is taken into account.

The second mechanism proposed in [19] to explain the large scattering length instead relies on a Feshbach resonance mechanism $[22,23]$ between the $D$ mesons and one charmonium level, specifically the $\chi_{c 1}(2 P)$. This situation is, however, probably too fortuitous to be taken seriously. First of all, the $\chi_{c 1}(2 P)$ has not been observed yet and secondly, potential models $[24,25]$ predict $\delta_{\chi_{c 1}(2 P)} \simeq 90 \mathrm{MeV}$. To bring it down to values compatible with the application of the Feshbach formalism would require quite large theoretical errors.

If one ignores for the moment the issues related to the prompt production, and assumes that the $X(3872)$ is indeed a purely molecular state, it is also rather immediate to explain the narrow width of this particle. The total width, $\Gamma_{X}$, will get contributions from both the decays of its constituents, $D^{*} \rightarrow D \pi$ and $D^{*} \rightarrow D \gamma$ (see e.g. Eq. (3.14)), and the decays into other hadronic states, like for example lower charmonia, $X \rightarrow \psi(2 S) \gamma$ or $X \rightarrow J / \psi \pi \pi$. However, since the states $|H\rangle$ in (3.16) are suppressed by $1 / a_{\mathrm{s}}$, the second possibility can be neglected and one remains with $\Gamma_{X} \approx \Gamma_{D^{*}} \simeq 65 \mathrm{keV}[26]$.

The scattering amplitude (3.1) can be rewritten in terms of the scattering length as

$$
f(a b \rightarrow a b) \equiv f(E)=-\frac{1}{\gamma+i \sqrt{2 m E}}
$$

where we have neglected the $i \epsilon$ regulator for convenience and defined $\gamma=1 / a_{\mathrm{s}}$. As already pointed out, if $\gamma>0, f(E)$ has a pole for $E<0$, in which case we are in presence of a real bound state. On the other hand, if $\gamma<0$ then the pole happens still for negative values of $E$, but on the unphysical Riemann sheet, and in this case we refer to a virtual (anti-bound) state. 
This is all strictly true for a stable shallow bound state. In order to take into account the finite width of the $X(3872)$, the authors of [26] allowed both the scattering length and the reduced mass that is implicit in $E$ to have an imaginary part:

$$
f(E)=-\frac{1}{\operatorname{Re} \gamma+i \operatorname{Im} \gamma+i \sqrt{2 m(E+\Gamma(E) / 2)}}
$$

with $\Gamma(E=0)=\Gamma_{D^{*}}$. While the latter one takes care of the direct decay of the $D^{*}$ constituent, Im $\gamma$ accounts for inelastic scattering processes. It can be argued that if the total energy $E$ is close to the $D^{0} \bar{D}^{* 0}$ threshold then the line shape $d \Gamma\left(B^{+} \rightarrow K^{+} D^{0} \bar{D}^{* 0}\right) / d E$ has energy dependence that only comes from the $|f(E)|^{2}$ factor. ${ }^{15}$ Using the 2003 data reported in [27] by the Belle collaboration, it was shown that the $\operatorname{Re} \gamma>0$ situation was favored with respect to the opposite one [26], hence suggesting for the $X$ to be a real, negative energy bound state — again, if the other difficulties of the molecular model are ignored.

\subsection{X(3872) as a deuteron-like state}

\subsubsection{Prompt production disfavors a pure molecule interpretation}

At very low energy, proton-neutron scattering $p n \rightarrow p n$, with $S=1$ as in deuteron, has a cross section

$$
\sigma_{p n}=\frac{2 \pi}{m B} \simeq 2.41 \mathrm{~b}
$$

using the binding energy $B=2.22 \mathrm{MeV}$ in deuteron. This agrees fairly well with the experimental value $\sim 3.6 \mathrm{~b}$. This does not mean that a bound state deuteron gets formed, which might only happen if the pn system looses energy and falls in the bound state. It is rather the manifestation of the enhancement in the scattering cross section due to the presence of a shallow bound state.

As suggested in the previous sections, the $X(3872)$ might be a deuteron-like object: a real bound state whose lifetime is given by the shorter lived component, the $D^{*}$. Are deuterons produced at LHC at large transverse momenta? How do their production rates compare with that of of $X(3872)$ ? If the nature of the two particles is indeed almost the same, then the behavior of the $X$ should not be too far away from that of the deuteron.

In a deuteron-like description of $X(3872)$ based on a (three-dimensional) square well potential of depth $V_{0} \simeq$ $6.7 \mathrm{MeV}$ and range $R \simeq 3 \mathrm{fm}$, a bound state energy $B=0.14 \mathrm{MeV}$ is obtained. The expectation value of the kinetic energy in the bound state $\psi$ is found to be

$$
\langle T\rangle_{\psi} \simeq 1.29 \mathrm{MeV} \quad\left(k_{\mathrm{rel}} \simeq 50 \mathrm{MeV}\right)
$$

with an average radius of $\langle r\rangle \simeq 10 \mathrm{fm} .{ }^{16}$ Note that the relative momentum has a rather higher value with respect to those discussed before. However we have to observe that, in this model, the $D$ and $\bar{D}^{*}$ mesons have indeed finite negative total energy, i.e. they are stably bound together. To make this happen, the initially free $\bar{D} D^{*}$ pair produced in $p p$ hadronization must interact with at least a third hadron, to change abruptly its relative kinetic energy and fall in the discrete (even though superficial) level of the attractive potential $[20,30]$ - see the discussion in Appendix A. The expected $X$ width would therefore be $\Gamma_{X} \equiv \Gamma_{D^{*}} \approx 65 \mathrm{keV}$, even at a binding energy as large as $B=0.14 \mathrm{MeV}$ (compare to Figure 2), for it would be a stable bound state whose lifetime coincides with the lifetime of the shortest lived component.

\footnotetext{
${ }^{15}$ For energies close to threshold the decay matrix elements gets a $f(E)$ resonant factor [26].

${ }^{16} \mathrm{Using}$ the central value of the latest measurement of the binding energy [29], $B=(3 \pm 192) \mathrm{keV}$, one would find $k_{\text {rel }} \simeq 20 \mathrm{MeV}$ and a humongous size of $\langle r\rangle \simeq 58 \mathrm{fm}$ !
} 

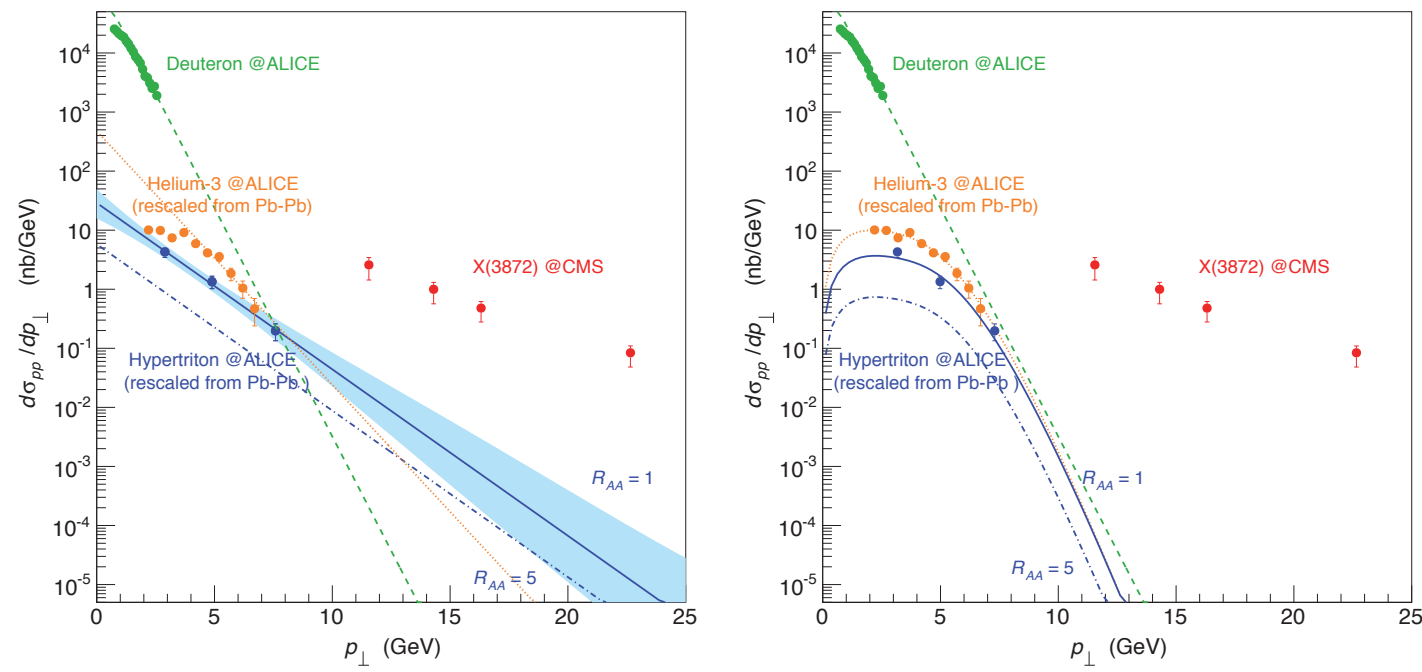

Figure 3: Comparison between the prompt production cross section in $p p$ collisions of $X(3872)$ (red), deuteron (green), helium (orange), and hypertriton (blue), from [28]. The $X$ data from CMS are rescaled by the branching ratio $\mathcal{B}(X \rightarrow J / \psi \pi \pi)$. Deuteron data in $p p$ collisions are taken from ALICE. The helium and hypertriton data measured by ALICE in $\mathrm{Pb}-\mathrm{Pb}$ collisions have been rescaled to $p p$ using a Glauber model. The dashed green line is the exponential fit to the deuteron data points in the $p_{\perp} \in[1.7,3.0] \mathrm{GeV}$ region, whereas the dotted orange one is the fit to the helium data points. The solid and dot-dashed blue lines represent the fits to hypertriton data with $R_{A A}=1$ (no medium effects) and an hypothetical constant value of $R_{A A}=5$. The hypertriton data points are horizontally shifted at the bin centers of gravity - being defined as the point at which the value of the fitted function equals the mean value of the function in the bin. Left Panel: the hypertriton data are fitted with an exponential curve, and the light blue band is the $68 \%$ C.L. for the extrapolated $R_{A A}=1$ curve. helium data in the $p_{\perp} \in[4.45,6.95] \mathrm{GeV}$ region are also fitted with an exponential curve. Right Panel: the hypertriton and helium data are fitted with blast-wave functions, whose parameters are locked to the helium ones.

We conclude that the formation of the $X$ resonance might occur either via a low energy resonant scattering phenomenon or via multi-body final state interactions producing a deuteron-like state whose metastability is provided by the shorter lived component in the system, namely the $D^{*}$ particle - energetic arguments do not suggest that the formation of the $\bar{D} D^{*}$ molecule stabilize the $D^{*}$ within the bound state.

The deuteron-like case, might be more realistic when considering $X$ prompt production in high energy $p p$ collisions at the LHC, with high transverse momentum cuts on hadrons. Indeed in this case it is very unlikely that the relative energy in the center of mass of the $\bar{D} D^{*}$ system is $E \sim B \sim 0$ as required by the shallow bound state formalism, especially when high transverse momentum $p_{\perp}$ cuts are considered. This latter point has thoroughly been discussed using Monte Carlo simulations [20, 30-32]: the main observation is that the relative momentum in the center of mass of the pair $k_{\text {rel }}$ has an average value $\left\langle k_{\text {rel }}\right\rangle \approx 1.3 p_{\perp}$ which grows with $p_{\perp}$ and the $X(3872)$ has been measured with high cross section at CMS with $p_{\perp} \gtrsim 12 \mathrm{GeV}$ — see Figure 3 [28]. For more details on this point see Appendix A.

On the other hand, if we wish to interpret the $X(3872)$ as a deuteron-like particle formed in hadronization, then we have to confront with data on production of deuteron, or other loosely bound light nuclei, in $p p$ collisions at high transverse momenta. In particular it is useful to compare the large production cross sections of $X(3872)$ observed by $\mathrm{CMS}$ at high $p_{\perp}$ with the extremely small deuteron production expected in the same region extrapolating recent data from the ALICE collaboration, shown in Figure 3. Data points at higher $p_{\perp}$ will be collected in the future to help this comparison. At any rate, the extrapolation of the data on deuteron, Helium-3 and hypertriton - all bona fide loosely bound molecules - clearly shows how the $X(3872)$ is too copiously produced at the vertex of high energy collisions to be easily interpreted as a meson molecule with tiny binding energy. Although more data will clearly provide an essential insight, it is our opinion that the comparison with the experiment speaks against a molecular interpretation of this resonance. 
Recently, it has been proposed that, at high $p_{T}$, the production of exotic states depends on the short-range nature, namely on the number of quarks which compose the state, regardless of their molecular or compact nature, so that prompt production could give no information about the nature of these states. This would agree with the predictions of counting rules [33-35]. However these rules are derived for the exclusive reactions only, where all the (relatively small, as discussed in [35]) center of mass energy is shared among few final hadrons, and do not apply to very high energy inclusive production of exotic states. In any case, in Figure 3 one can appreciate that the slope of the deuteron is much steeper than the one of hypertriton, which is at odds with the naïve counting (6 quarks versus 9 quarks). The short-range nature of the production would also be inconsistent with the use of final state interactions to get the enhancement of two orders of magnitude needed to reach the experimental value of the cross section [36-39] — see also Appendix A.

\subsubsection{Production through $c \bar{c}$}

An alternative idea that can justify the $X(3872)$ prompt production cross section has been proposed in [40, 41]. As already explained in Sec. 3.2, the $X(3872)$ can a priori be represented by a superposition of all the hadronic states with the right $J^{P C}=1^{++}$quantum numbers.

The authors of $[40,41]$ express the $X(3872)$ state as

$$
|X\rangle=\sqrt{Z_{c \bar{c}}}\left|\chi_{c 1}(2 P)\right\rangle+\sqrt{Z_{\mathrm{mol}}}\left|D D^{*}\right\rangle
$$

where states with a different number of 'valence' quarks are superimposed.

Here the state $\left|D D^{*}\right\rangle$ is intended as summed over momenta - see Eq. (3.16). The observables like the prompt production, the $b$-production and the quark annihilating decays can be explained through the charmonium component, while the hadronic decays into $D D \pi, D D \gamma, J / \psi \rho$ and $J / \psi \omega$ through the molecular one.

The results of the two papers are in agreement. Here we will follow the notation used in [41]. The authors use the Non-Relativistic QCD (NRQCD) approach [42] at Next-to-Leading Order (NLO), which has proven to be quite successful in reproducing the yields of $J / \psi[43]$ and $\chi_{c J}(1 P)$ [44] at Tevatron and LHC. The production cross section of the $X(3872)$ in the $J / \psi \pi^{+} \pi^{-}$mode can then be written as

$$
d \sigma\left(p p \rightarrow X\left(J / \psi \pi^{+} \pi^{-}\right)\right)=d \sigma\left(p p \rightarrow \chi_{c 1}(2 P)\right) Z_{c \bar{c}} \mathcal{B}\left(X \rightarrow J / \psi \pi^{+} \pi^{-}\right)
$$

while the cross section for the production of the charmonium can be written using the NRQCD factorization as

$$
d \sigma\left(p p \rightarrow \chi_{1 c}(2 P)\right)=\sum_{i, j, n} \int d x_{1} d x_{2} G_{i / p}\left(x_{1}\right) G_{j / p}\left(x_{2}\right) d \widetilde{\sigma}\left(i j \rightarrow(c \bar{c})_{n}\right)\left\langle O_{n}^{\chi_{c 1}(2 P)}\right\rangle
$$

In the previous expression $i$ and $j$ enumerate the partons, and run over gluons, light quarks $(u, d, s)$ and antiquarks. Moreover, $G_{i / p}(x)$ is the Parton Distribution Function (PDF) of the proton and $d \widetilde{\sigma}$ is the partonic cross section. The collective index $n$ instead contains color, spin and angular momentum of the intermediate $c \bar{c}$ pair, and $\left\langle O_{n}^{\chi_{c 1}(2 P)}\right\rangle$ is a long distance matrix element embedding the conversion from $c \bar{c}$ to an actual charmonium. At NLO we only have $n={ }^{3} P_{1}^{\left[\mathbf{1}_{c}\right]},{ }^{3} S_{1}^{\left[\mathbf{8}_{c}\right]}$.

The authors of [41] then use the available data from CMS [45] to fit the free parameters of Eq. (3.26), and successfully reproduce CDF data $[46]^{17}$. They show that both the prompt production and the production from $B$ decays can be explained with a large weight for the $\chi_{c 1}(2 P)$ component estimated to be

$$
Z_{c \bar{c}}=(28-44) \% \text {. }
$$

\footnotetext{
${ }^{17}$ The prediction obtained from the fit underestimate the LHCb data [47] by a factor of 2, which might be due to a large theoretical uncertainty.
} 
Nevertheless there is no reason not to include in the state (3.24) a compact diquarkonium state (see Sec. 4) or any compact tetraquark state. It is more natural to superimpose states having an equal number of 'valence' quarks rather than states with an increasing number quarks as in (3.24). A compact tetraquark content would as well account for both the production and decays of the $X(3872)$, resulting in a simpler description which is able to explain the same observables. We remind here that the model for baryon production (incorporated in shower Monte Carlo libraries and generally used to study hadron collisions) is based on the diquark-quark color neutralization. In that case no meson-meson component would be needed.

\subsection{Consequences of the Heavy Quark Spin Symmetry}

The results obtained in the previous section for shallow bound states apply to every type of molecule. Also Heavy Quark Spin Symmetry (HQSS) can be used to make universal predictions. It arises in systems with heavy $Q$ and light quarks in the $m_{Q} \rightarrow \infty$ limit, with $Q=\{c, b\}$. In fact, the hyperfine interaction between the spin of the heavy quark and the chromomagnetic field generated by the light degrees of freedom appears at $O\left(1 / m_{Q}\right)$ in the Heavy Quark Effective Theory (HQET) Lagrangian. Consequently, in the $m_{Q} \rightarrow \infty$ limit, the properties of heavy mesons and quarkonia (e.g. their masses) are independent of the heavy spin. Light quarks are insensitive to the spin and flavor of the heavy quark, which behaves as a spectator. ${ }^{18}$ In particular, since pseudoscalar and vector heavy mesons and quarkonia are degenerate at lowest order, they can be collected in spin multiplets, e.g. $\left(D, D^{*}\right),\left(B, B^{*}\right),\left(\eta_{c}, J / \psi\right)$ and $\left(\eta_{b}, \Upsilon\right)$. This symmetry holds for heavy meson molecules as well, and can be employed to study their spectrum and decay modes, independently of the details of the potential. Corrections to this approximations are of order $O\left(\Lambda_{Q C D} / m_{Q}\right)$, i.e. $\sim 25 \%$ for the charm and $\sim 10 \%$ for the bottom, if constituent quark masses are considered.

To lowest order, the spin of the heavy quark and that of the light one are completely decoupled. When this happens, the Hilbert spaces of the heavy quark spinors and of the remaining degrees of freedom are completely separated. Consequently, for pseudoscalar $(P)$ and vector $(V)$ heavy-light mesons, the total wave function can be written as the direct product of the heavy quark spinor times the wave function of the remaining degrees of freedom

$$
\Psi_{P}=\bar{\psi}_{q} \sigma^{2} \chi_{Q} \quad \text { and } \quad \boldsymbol{\Psi}_{V}=\bar{\psi}_{q} \sigma^{2} \boldsymbol{\sigma} \chi_{Q}
$$

where $\chi_{Q}$ is the Pauli two-dimensional spinor of the heavy quark, $\bar{\psi}_{q}$ is the wave function of the bound light antiquark and the spinless heavy quark and $\sigma$ are the Pauli matrices, suitable to spin-1 states. Note that we are using a nonrelativistic formalism, and hence the antiparticles are independent of the particles, i.e. $\bar{\psi}_{q} \neq \psi_{q}^{\dagger} .{ }^{19}$ This implies that no creation/annihilation of pairs is allowed. In general $\bar{\psi}_{q}(\boldsymbol{r})$ will be a complicated object containing information about everything but the spin of the heavy quark, e.g. spinor of the light antiquark, spatial distribution of the $Q \bar{q}$ pair, etc. Note also that with our notation, the wave functions for the respective antimesons are [48]

$$
\bar{\Psi}_{P}=\bar{\chi}_{Q} \sigma^{2} \psi_{q} \quad \text { and } \quad \overline{\mathbf{\Psi}}_{V}=\bar{\chi}_{Q} \sigma^{2} \sigma \psi_{q}
$$

Since the spin-flip of the heavy degree of freedom is suppressed by powers of $1 / m_{Q}$, the spin of the $Q \bar{Q}$ pair can be used as a "label" for the state. If one assumes the $X, Z_{Q}$ and $Z_{Q}^{\prime}$ to be molecular states, then using the SU(2) Fierz

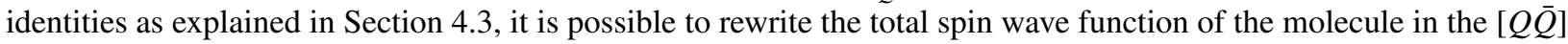
and $\left[q \bar{q}^{\prime}\right]$ basis rather than in the $[q \bar{Q}]$ and $\left[Q \bar{q}^{\prime}\right]$ one. If in Eqs. (3.28) and (3.29) we only focus on the spin part of the full wave function, it is possible to find [48, 49]

$$
\begin{aligned}
X & \sim \overline{\boldsymbol{\Psi}}_{V} \Psi_{P}-\bar{\Psi}_{P} \boldsymbol{\Psi}_{V} \sim\left|1_{Q \bar{Q}}, 1_{q \bar{q}}\right\rangle \\
Z_{Q} & \sim \overline{\mathbf{\Psi}}_{V} \Psi_{P}+\bar{\Psi}_{P} \boldsymbol{\Psi}_{V} \sim\left|0_{Q \bar{Q}}, 1_{q \bar{q}^{\prime}}\right\rangle+\left|1_{Q \bar{Q}}, 0_{q \bar{q}^{\prime}}\right\rangle \\
Z_{Q}^{\prime} & \sim \overline{\boldsymbol{\Psi}}_{V} \times \boldsymbol{\Psi}_{V} \sim\left|0_{Q \bar{Q}}, 1_{q \bar{q}^{\prime}}\right\rangle-\left|1_{Q \bar{Q}}, 0_{q \bar{q}^{\prime}}\right\rangle
\end{aligned}
$$

\footnotetext{
${ }^{18}$ This is exactly analogous to ordinary atomic physics where, at the lowest level, the heavy nucleus just provides a static source for the Coulomb field.

${ }^{19} \psi_{q}$ only contains annihilation operators for the particle.
} 
where by $S_{q_{1} q_{2}}$ we represent the spin of the $q_{1} q_{2}$ pair, $P=(D, B)$ and $V=\left(D^{*}, B^{*}\right)$.

The previous spin decompositions can be used to extract selection rules for the decay of the would-be meson molecules into quarkonia. For example, one readily deduces that the hadronic transition $X \rightarrow \eta_{c} \pi \pi$ should be suppressed because the spin of the $\eta_{c}$ is $S_{c \bar{c}}=0$, while the decay into spin-1 charmonia should be favored. In [49], Eq. (3.30a) is used to estimate the ratio between the widths $\Gamma\left(X \rightarrow \pi^{0} \chi_{c 1}\right)$ and $\Gamma\left(X \rightarrow \pi^{+} \pi^{-} J / \psi\right)$,

$$
\frac{\Gamma\left(X \rightarrow \pi^{0} \chi_{c 1}\right)}{\Gamma\left(X \rightarrow \pi^{+} \pi^{-} J / \psi\right)} \approx 0.35\left(\frac{0.5 \mathrm{GeV}}{\mu}\right)^{2}
$$

where $\mu$ is a dimensional factor that takes into account the $P$-wave emission of the single $\pi^{0}$. The $X$ has, in fact, $J^{P}=1^{+}$while the $\pi^{0}$ and $\chi_{c 1}$ are $J^{P}=0^{-}$and $1^{+}$respectively. Therefore, in order to conserve both angular momentum and parity the decay must be in $P$-wave. From the above equation it is clear that for reasonable values of $\mu$ the decay into $\pi^{0} \chi_{c 1}$ should have an observable rate.

In [48] HQSS is also employed to make several predictions about the $Z_{b}$ and $Z_{b}^{\prime}$ states. These considerations clearly apply to the charm sector as well, albeit with larger uncertainties. First of all, Eqs. (3.30b) and (3.30c) show that the magnitude of the couplings to $J=0$ and $J=1$ bottomonia are the same in both the $Z_{b}$ and the $Z_{b}^{\prime}$. Moreover, in the $m_{b} \rightarrow \infty$ limit, the $0_{b \bar{b}}$ and $1_{b \bar{b}}$ bottomonia are degenerate, as well as the $Z_{b}$ and $Z_{b}^{\prime}$. It then follows that the two $Z_{b}^{(\prime)}$ states are expected to decay into degenerate states with lower masses and their widths should be roughly the same, $\Gamma\left(Z_{b}\right) \approx \Gamma\left(Z_{b}^{\prime}\right)$. Moreover, since the combinations (3.30b) and (3.30c) are orthogonal, the decay $Z_{b}^{\prime} \rightarrow \bar{B} B^{*}$ is forbidden. Both these predictions are in good agreement with the data. In [48] considerations about the $\Upsilon(5 S) \rightarrow \Upsilon(n S) \pi^{+} \pi^{-}$ process as compared to the $\Upsilon(5 S) \rightarrow h_{b}(k P) \pi^{+} \pi^{-}$were also made. In particular, even though the coupling to $J=0$ and $J=1$ bottomonia have the same magnitude, they have opposite sign. This is manifest in the interference pattern for the two decay previously mentioned.

In [50] the HQSS was employed for the study of the $Y(4660)$. Because of its decay into $\psi(2 S) \pi^{+} \pi^{-}$, it is interpreted in the molecular picture as a $\psi(2 S) f_{0}(980)$ bound state. ${ }^{20}$ The main consequence is the expected existence of the other state of the heavy spin multiplet, namely the $Y_{\eta}=\eta_{c}^{\prime} f_{0}(980)$. In particular, it can be estimated [50] that the branching ratio for the $B^{+} \rightarrow \eta_{c}^{\prime} K^{+} \pi^{+} \pi^{-}$process is of order $10^{-3}$ and hence it should be very likely to observe the $Y_{\eta}$ in the $\eta_{c}^{\prime} \pi^{+} \pi^{-}$final state of the previous decay at Belle and BABAR.

\subsection{An effective field theory approach to heavy meson molecules}

Another potential-independent tool for the study of the decays of loosely bound molecules has been developed and employed in [52-58]. It is a Non-Relativistic Effective Field Theory (NREFT) obtained from the time-honored Heavy Meson Chiral Theory [59]. The work on this topic is copious and it has been applied mostly to the prediction of the branching fractions for the radiative and hadronic decays of the $X Y Z$ resonances.

The hypothesis about the molecular nature of the states is implemented in the model by forcing the exotic resonances to couple to their constituents only, as a consequence of Weinberg's compositeness theorem $[14,60]$. It then follows that every decay into a final state different from the constituents themselves must happen via a heavy meson loop. In Figure 4 we report an example of such process for the $Z_{c} \rightarrow J / \psi \pi$ decay.

The typical velocity of the heavy mesons involved in the previous loops is

$$
v \approx \sqrt{\frac{\left|M_{X}-2 m\right|}{m}}
$$

\footnotetext{
${ }^{20}$ The same authors consider the $f_{0}(980)$ to be a $K \bar{K}$ molecule [51]. The $Y(4660)$ would be a sort of 'matryoshka' molecule, where the interplay of the different scales involved is not clear.
} 

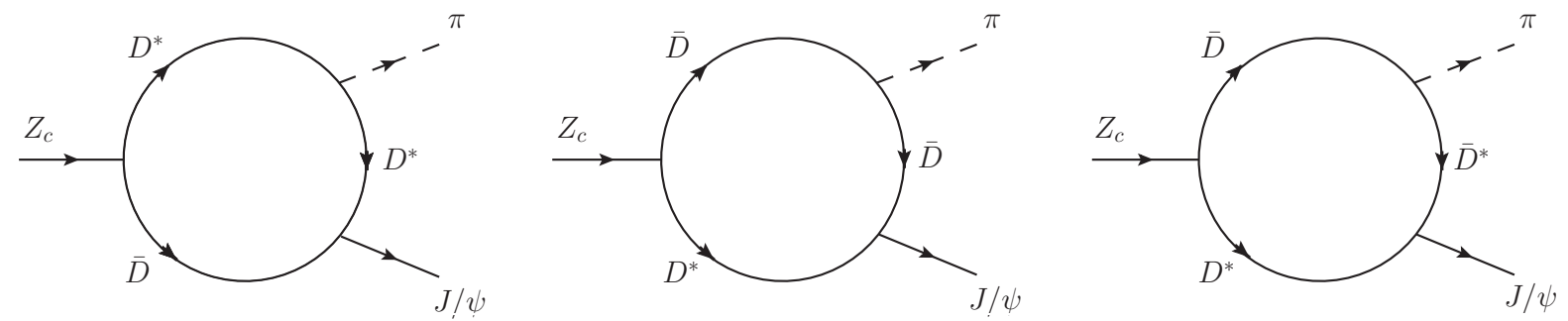

Figure 4: Typical heavy meson loop used in NREFT to describe the decay of the exotic states. In this figure the $Z_{c} \rightarrow J \psi \pi$ decay happens through an intermediate $D D^{*}$ loop, as imposed by the molecular nature of the $Z_{c}(3900)$.

where $M_{X}$ is the mass of the exotic states and $m$ that of the open flavor mesons. Being the former ones very close to threshold, such velocities are small and hence allow for a non-relativistic treatment. This is done by letting $v_{\mu} \rightarrow(1, \mathbf{0})$ in the HQET bi-spinors (see Section 3.6.1 below for their definitions). As a result of this procedure, the interaction of the $X Y Z$ states with heavy pseudoscalar and vector mesons is described by the following terms

$$
\begin{aligned}
\mathcal{L}_{X} & =\frac{g_{X}}{\sqrt{2}} X^{i \dagger}\left(\bar{P} V^{i}-P \bar{V}^{i}\right)+\text { h.c. } \\
\mathcal{L}_{Z_{Q}} & =\frac{g_{Z_{Q}}}{\sqrt{2}} Z_{Q}^{i \dagger}\left(\bar{P} V^{i}+P \bar{V}^{i}\right)+\text { h.c. } \\
\mathcal{L}_{Z_{Q}^{\prime}} & =i g_{Z_{Q}^{\prime}} \epsilon_{i j k}\left(Z_{Q}^{\prime}\right)^{i \dagger} \bar{V}^{j} V^{k}+\text { h.c. }
\end{aligned}
$$

The fields $\boldsymbol{X}$ and $\boldsymbol{Z}_{Q}^{(\prime)}$ annihilate the exotic meson states, while the $P(\bar{P})$ and $\boldsymbol{V}(\overline{\boldsymbol{V}})$ annihilate the pseudoscalar and vector (anti)mesons respectively. In particular, the normalization of the states is such that $P|P(p)\rangle=\sqrt{m_{P}}|0\rangle$ and $V^{i}|V(p, \lambda)\rangle=\lambda^{i} \sqrt{m_{V}}|0\rangle$, where $\lambda$ is a polarization vector. The indices $i, j$ and $k$ are only spatial, as a consequence of the non-relativistic limit. The $g_{i}$ 's are effective strong couplings to be fitted from the data - see e.g. [54, 56, 57].

The smallness of the velocities also allows to substitute the relativistic heavy mesons propagator with its nonrelativistic version

$$
\frac{i}{p^{2}-m^{2}+i \epsilon} \longrightarrow \frac{1}{2 m} \frac{i}{p^{0}-\frac{p^{2}}{2 m}-m+i \epsilon}
$$

It is important to note that the effective couplings can be large and hence it is not obvious a priori that including one-loop diagrams only is enough. However, since $v$ is here a small parameter, one can indeed apply a power counting procedure to estimate the relevance of higher order processes and consequently determine the applicability of perturbation theory. In particular, heavy meson loops imply a non-relativistic integral over $d p^{0} d^{3} p /(4 \pi)^{2}$ and hence count as $v^{5} /(4 \pi)^{2}$ while the propagator in Eq. (3.34) contributes with a $1 / v^{2}$, given that the states are close to threshold. Lastly, if the interaction vertices in (3.33) contain derivatives they will come with either an additional power of $v$ or of the outgoing momenta $q$, depending if the derivative acts on the heavy meson running in the loop or on the external legs.

This NREFT has been employed in [57] to shown that the branching ratios for the $Z_{c}^{(\prime)} \rightarrow \eta_{c} \rho$ decays can provide a powerful tool of discrimination between meson molecule and compact tetraquark. In Figure 5 we report the likelihood distributions for the following ratios

$$
\mathcal{R}_{Z}=\frac{\mathcal{B}\left(Z_{c} \rightarrow \eta_{c} \rho\right)}{\mathcal{B}\left(Z_{c} \rightarrow J / \psi \rho\right)} \quad \text { and } \quad \mathcal{R}_{Z^{\prime}}=\frac{\mathcal{B}\left(Z_{c}^{\prime} \rightarrow \eta_{c} \rho\right)}{\mathcal{B}\left(Z_{c}^{\prime} \rightarrow h_{c} \pi\right)}
$$

computed using the two models. As one immediately sees, the predictions differ from each other by more than $2 \sigma$ and hence the $\eta_{c} \rho$ channel can be used as an experimental tool to probe the internal structure of the charged $Z_{c}^{(\prime)}$ states. The same authors fitted the couplings $g_{Z_{c}}$ and $g_{Z_{c}^{\prime}}$ from data, assuming for the total width to be saturated by 

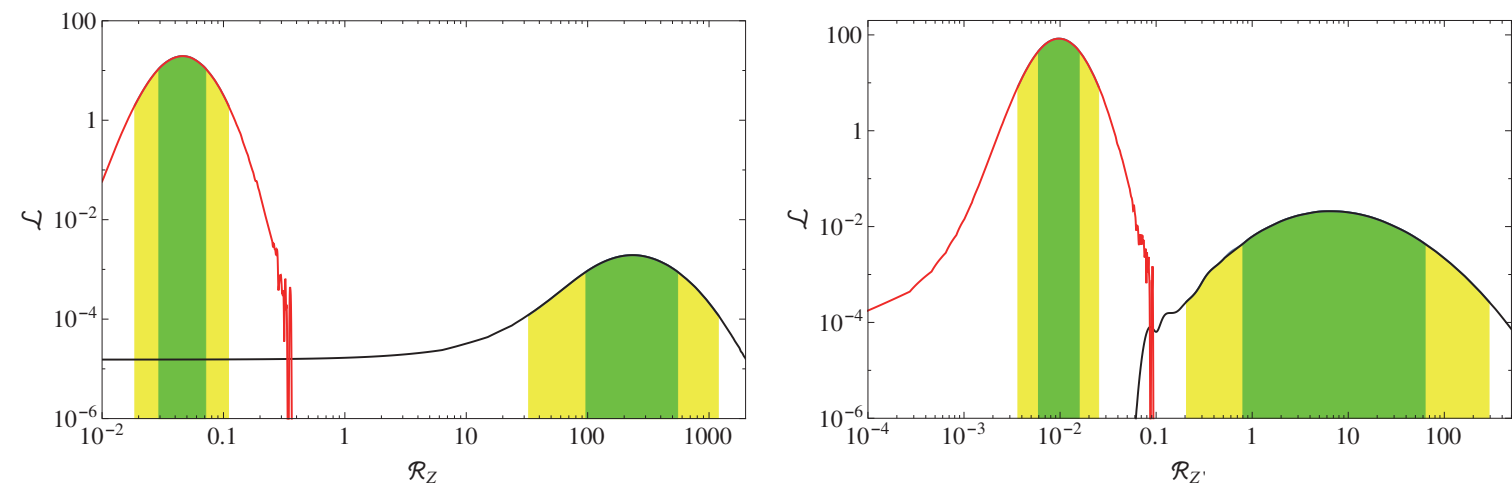

Figure 5: Likelihood distributions for the ratios in Eq. (3.35) as computed in [57]. The red line corresponds to the results obtained using the NREFT for the molecular model, while the black one corresponds to the diquarkonium picture. The yellow (green) band corresponds to the $68 \%$ (95\%) confidence region. The two models provide significantly different predictions.

the $D^{(*)} D^{*}, \eta_{c} \rho, h_{c} \pi J / \psi \pi$ and $\psi(2 S) \pi$ final states. Once the couplings are known one can compute the ratios for the decays of the two different $Z_{c}^{(\prime)}$ into the same final state, assuming molecular nature. One finds

$$
\frac{\mathcal{B}\left(Z_{c} \rightarrow h_{c} \pi\right)}{\mathcal{B}\left(Z_{c}^{\prime} \rightarrow h_{c} \pi\right)}=0.34_{-0.13}^{+0.21} \quad \text { and } \quad \frac{\mathcal{B}\left(Z_{c} \rightarrow J / \psi \pi\right)}{\mathcal{B}\left(Z_{c}^{\prime} \rightarrow J / \psi \pi\right)}=0.35_{-0.21}^{+0.49}
$$

The previous estimates essentially tell us that, within theoretical errors, the branching fractions for the $Z_{c}$ and $Z_{c}^{\prime}$ into the same final state should be of the same order of magnitude. Both should be observed in both channels. The $h_{c} \pi$ channel might indeed be compatible with experimental data, where a hint of $Z_{c}$ is seen (albeit not statistically significant). On the other hand the $Z_{c}^{\prime}$ is completely missing from the $J / \psi \pi$ final state, which seems to be in contrast with the molecular prediction — see Section 8.2.

A slightly different approach has been used in $[19,52,58]$ to describe the $X(3872)$. In this case, the interaction Lagrangian does not contain the $\boldsymbol{X}$ field explicitly but it only describes the interaction of the $D$ mesons with pions and charmonia. Instead of requiring the presence of intermediate open flavor meson loops as explained above, the authors automatically implement the molecular hypothesis by describing the $X(3872)$ with the interpolating field $\boldsymbol{X}=(D \overline{\boldsymbol{D}}-\bar{D} \boldsymbol{D}) / \sqrt{2}$. This method has been used to describe the $X \rightarrow \bar{D}^{0} D^{0} \pi^{0}[52]$ and $X \rightarrow \chi_{c J} \pi^{0}$ [58] decays, as well as to provide an explicit implementation of the fine tuning required to explain the unnaturally large scattering length of the $X(3872)$ [19] — see Section 3.2 above.

\subsection{Phenomenological realizations of the meson-meson potential}

Most of the considerations made so far about loosely bound molecules follow strictly from the application of the theory of shallow bound states, or of the HQSS. The results obtained in the two cases are universal in the $B \rightarrow 0$ and $m_{Q} \rightarrow \infty$ limits respectively, as they do not rely on the detailed knowledge of the binding potential, but rather on the mere existence of a (loosely) bound state. The actual interaction between the two mesons is the complicated result of residual strong forces between color singlet objects. It then follows that its description must rely on some phenomenological picture and approximations, which necessarily introduce a good degree of model dependence in the final results, e.g. the value of the binding energy or even the very existence of bound states. Here we introduce the most popular models for the explicit realization of the inter-hadron potential. 


\subsubsection{One-pion-exchange}

It is well known that the force that holds the deuteron together can be described by a potential whose main contribution comes from the exchange of a pion between the two nucleons. With this in mind, it is rather natural to try to extend the same approach to mesons and see whether or not this so-called one-pion-exchange potential allows for bound states. This program was first carried on in [61, 62], mainly for the study of light mesons and extended to the heavy sector by several different authors, e.g. [57, 63-66]. It was already pointed out in the original references that using only this kind of potential could only provide plausibility arguments since the full treatment would require the inclusion of coupled channel effects, two-pion-exchange, etc.

The leading order contribution to this potential comes from the exchange of a single pion between the two mesons. Although the result is true also for light mesons, as computed in [62], here we will derive the potential starting from the HQET formalism (see e.g. [59, 67]). It is well known that in the $m_{Q} \rightarrow \infty$ limit, the interaction between the light pseudoscalar mesons and the heavy mesons can be described by the following Lagrangian

$$
\mathcal{L}=-\frac{g}{f_{\pi}} \operatorname{tr}\left[\bar{H}_{a} H_{b} \gamma_{\mu} \gamma_{5}\right] \partial^{\mu} \mathcal{M}_{a b}
$$

where $f_{\pi} \simeq 132 \mathrm{MeV}$ is the pion decay constant and $g$ is some dimensionless axial coupling (typically around $0.5-$ 0.7 [59]). The expression is traced over Dirac indices. $H_{a}$ is a bi-spinor containing the operators $V_{a \mu}$ and $P_{a}$ describing respectively the vector and pseudoscalar heavy mesons as

$$
H_{a}=\frac{1+\psi}{2}\left[V_{a \mu} \gamma^{\mu}-P_{a} \gamma_{5}\right] \quad \text { and } \quad \bar{H}_{a}=\gamma_{0} H_{a}^{\dagger} \gamma_{0}
$$

where $a$ and $b$ are flavor indices. The light pseudo-Goldstone boson fields are instead contained in

$$
\mathcal{M}=\left(\begin{array}{ccc}
\frac{1}{\sqrt{2}} \pi^{0}+\frac{1}{\sqrt{6}} \eta_{8} & \pi^{+} & K^{+} \\
\pi^{-} & -\frac{1}{\sqrt{2}} \pi^{0}+\frac{1}{\sqrt{6}} \eta_{8} & K^{0} \\
K^{-} & \bar{K}^{0} & -\sqrt{\frac{2}{3}} \eta_{8}
\end{array}\right)
$$

The interaction in Eq. (3.37) can then be rewritten as

$$
\mathcal{L}=-\frac{2 g}{f_{\pi}}\left(V_{\mu} \partial^{\mu} \mathcal{M} P^{\dagger}+\text { h.c. }\right)+\frac{2 i g}{f_{\pi}} \epsilon_{\alpha \beta \mu \nu} V^{\beta} \partial^{\mu} \mathcal{M} V^{\dagger \alpha} v^{v}
$$

where we recall that $v^{v}$ is the HQET four-velocity appearing in Eq. (3.38). From the previous equations one can find the nonrelativistic momentum space potentials for the exchange of a pion between vector and pseudoscalar heavy mesons

$$
\begin{gathered}
V_{\pi}(V V)=-V_{\pi}(V \bar{V})=\frac{8 g^{2}}{f_{\pi}^{2}}\left(\boldsymbol{\tau}_{1} \cdot \boldsymbol{\tau}_{2}\right)\left(\boldsymbol{\Sigma}_{1} \cdot \boldsymbol{q}\right)\left(\boldsymbol{\Sigma}_{2} \cdot \boldsymbol{q}\right) \frac{1}{\boldsymbol{q}^{2}+m_{\pi}^{2}} \\
V_{\pi}(P V)=\frac{8 g^{2}}{f_{\pi}^{2}}\left(\boldsymbol{\tau}_{1} \cdot \boldsymbol{\tau}_{2}\right)\left(\mathbb{1}_{1} \cdot \boldsymbol{q}\right)\left(\mathbb{1}_{2} \cdot \boldsymbol{q}\right) \frac{1}{\boldsymbol{q}^{2}+\mu^{2}}
\end{gathered}
$$

to be sandwiched between appropriate polarization vectors. Here $\boldsymbol{\tau}_{i}$ are isospin Pauli matrices, $\boldsymbol{\Sigma}_{i}$ and $\mathbb{1}_{i}$ are spin-1 matrices, and $\mu^{2}=m_{\pi}^{2}-\left(m_{V}-m_{P}\right)^{2}$. The definition of $\mu$ is supposed to approximately take into account recoil effects due the different masses of the constituents. ${ }^{21}$ Note that $P P$ interactions in the one-pion-exchange approximation are forbidden by parity conservation.

\footnotetext{
${ }^{21}$ This prescription is meaningful as long as $\mu^{2}$ is not too far from $m_{\pi}^{2}$ as, for example, in the bottom case. Less obvious is its validity in the charm sector where $m_{D^{*}}-m_{D} \approx m_{\pi}$, or even for $\bar{K} K^{*}$ molecules, where $\mu^{2}<0$.
} 
The two potentials are both in the form

$$
V_{\pi}(\boldsymbol{q})=\frac{8 g^{2}}{f_{\pi}^{2}}\left(\boldsymbol{\tau}_{1} \cdot \boldsymbol{\tau}_{2}\right)\left(\mathcal{A}_{1} \cdot \boldsymbol{q}\right)\left(\mathcal{A}_{2} \cdot \boldsymbol{q}\right) \frac{1}{\boldsymbol{q}^{2}+m^{2}}
$$

with obvious definitions. The position space potential is therefore given by their Fourier transform

$$
\begin{aligned}
V_{\pi}(\boldsymbol{x}) & =\frac{8 g^{2}}{f_{\pi}^{2}}\left(\boldsymbol{\tau}_{1} \cdot \boldsymbol{\tau}_{2}\right) \int \frac{d^{3} q}{(2 \pi)^{3}}\left(\mathcal{A}_{1} \cdot \boldsymbol{q}\right)\left(\mathcal{A}_{2} \cdot \boldsymbol{q}\right) \frac{e^{i \boldsymbol{q} \cdot \boldsymbol{x}}}{\boldsymbol{q}^{2}+m^{2}}=-\frac{8 g^{2}}{f_{\pi}^{2}}\left(\boldsymbol{\tau}_{1} \cdot \boldsymbol{\tau}_{2}\right)\left(\mathcal{A}_{1 i} \cdot \partial_{i}\right)\left(\mathcal{A}_{2 j} \cdot \partial_{j}\right) \int \frac{d^{3} q}{(2 \pi)^{3}} \frac{e^{i \boldsymbol{q} \cdot \boldsymbol{x}}}{\boldsymbol{q}^{2}+m^{2}} \\
& =-\frac{8 g^{2}}{f_{\pi}^{2}}\left(\boldsymbol{\tau}_{1} \cdot \boldsymbol{\tau}_{2}\right)\left(\mathcal{A}_{1 i} \cdot \partial_{i}\right)\left(\mathcal{A}_{2 j} \cdot \partial_{j}\right) \frac{e^{-m r}}{4 \pi r}
\end{aligned}
$$

Now, recalling that $\partial^{2}(1 / r)=-4 \pi \delta^{(3)}(\boldsymbol{x})$, we obtain the one-pion-exchange potentials in configuration space

$$
V_{\pi}(\boldsymbol{x})=-\frac{8 g^{2}}{f_{\pi}^{2}}\left(\boldsymbol{\tau}_{1} \cdot \boldsymbol{\tau}_{2}\right) \mathcal{A}_{1 i} \mathcal{A}_{2 j}\left\{\left[\left(3 \frac{x_{i} x_{j}}{r^{2}}-\delta_{i j}\right)\left(1+\frac{3}{m r}+\frac{3}{m^{2} r^{2}}\right)+\delta_{i j}\right] \frac{m^{2}}{3} \frac{e^{-m r}}{r}-\frac{4 \pi}{3} \delta_{i j} \delta^{(3)}(\boldsymbol{x})\right\}
$$

Because of the singular behavior at $r=0$ the previous potential needs to be regularized. This is typically done introducing some form factor for the $\pi V$ and $\pi P$ vertices, which also allows to neglect the $\delta$-function contribution. At any rate, this short term contribution could be ignored since one-pion exchange is not a fully reliable model for meson-meson short range interactions. Once the potential is specified it is just a matter of numerically solving the Schrödinger equation and verify whether a bound state is present or not.

In the very first attempt [61], the author tried to identify the observed $f_{1}(1420), f_{0}(1710), f_{2}^{\prime}(1525)$ and $f_{0}(1500)$ mesons with respectively $K \bar{K}^{*}, K^{*} \bar{K}^{*}, \omega \omega$ and $\rho \rho$ bound states. However, the numerical analysis later performed [62] showed that, although attractive, one-pion-exchange potentials alone are not enough to bind light mesons molecules. On the other hand, they are allowed in the heavy sector. In particular, charm bound states are predicted to be very near threshold while their bottom counter part should have a binding energy around $50 \mathrm{MeV} .{ }^{22}$ The detailed analysis of the allowed bound states and their quantum numbers, in the heavy quark sector is carried out in [62, 65]. The results are reported in Table 2 . One immediately notices that there are good candidates for the $X$, the $Z_{c}^{\prime}$ and $Z_{b}^{\prime}$ (even though the mass of the last one does not comply with experimental data) while there are no bound states corresponding to the $Z_{c}$ and $Z_{b}$. The latter ones should, in fact, be $P \bar{V}$ molecules but with $J^{P C}=1^{+-}$.

The absence of observed bound states from the present model does not rule out the molecular interpretation of the exotic resonances. It rather means that the long range, pion mediated interaction alone, as captured by the present model, cannot describe the observed spectrum. A contribution from short range potential is therefore needed. Such interactions are, however, complicated and their phenomenological realization can hardly be deduced from first principles. In the following section we will report one of this attempts.

In [66] it was also observed that because of the $\tau_{1} \cdot \tau_{2}$ term in Eqs. (3.41), the potential for a given molecule made of isospin-1/2 mesons, and with total isospin $I$ and third component $I_{3}$, comes with an overall factor

$$
C\left\langle I, I_{3}\left|\tau_{1} \cdot \tau_{2}\right| I, I_{3}\right\rangle=2 C\left(I(I+1)-\frac{3}{2}\right)
$$

where $C$ is the eigenvalue of the charge conjugation. This means that in the one-pion-exchange approximation, if the $I=0$ channel is attractive the $I=1$ is repulsive and viceversa. Hence, for a given set of quantum numbers, isosinglets and isovectors are unlikely to coexist. Indeed, in the case of the $X(3872)$ assumed as an isosinglet, we have $C=+$, $I=0$, which is attractive. For the almost degenerate $Z_{c}(3900)$, we have $C=-, I=1$, which is still attractive, although

\footnotetext{
${ }^{22}$ In [68] it was also noticed that, even in presence of an attractive one-pion-potential, meson-meson bound states are only allowed for sufficiently large reduced mass.
} 


\begin{tabular}{ccc|ccc}
\hline \hline Constituents & $J^{P C}$ & Expected mass $(\mathrm{MeV})$ & Constituents & $J^{P C}$ & Expected mass $(\mathrm{MeV})$ \\
\hline$D \bar{D}^{*}$ & $0^{-+}$ & $\simeq 3870$ & $B \bar{B}^{*}$ & $0^{-+}$ & $\simeq 10545$ \\
$D \bar{D}^{*}$ & $1^{++}$ & $\simeq 3870$ & $B \bar{B}^{*}$ & $1^{++}$ & $\simeq 10562$ \\
\hline$D^{*} \bar{D}^{*}$ & $0^{++}$ & $\simeq 4015$ & $B^{*} \bar{B}^{*}$ & $0^{++}$ & $\simeq 10582$ \\
$D^{*} \bar{D}^{*}$ & $0^{-+}$ & $\simeq 4015$ & $B^{*} \bar{B}^{*}$ & $0^{-+}$ & $\simeq 10590$ \\
$D^{*} \bar{D}^{*}$ & $1^{+-}$ & $\simeq 4015$ & $B^{*} \bar{B}^{*}$ & $1^{+-}$ & $\simeq 10608$ \\
$D^{*} \bar{D}^{*}$ & $2^{++}$ & $\simeq 4015$ & $B^{*} \bar{B}^{*}$ & $2^{++}$ & $\simeq 10602$ \\
\hline \hline
\end{tabular}

Table 2: Bound states allowed by the one-pion-exchange potential as found in [65]. All state have $I=0$.

with a three times smaller potential. Indeed it was for a long time considered too weak to allow for a bound state. The argument was withdrawn as soon as the charged states were observed. This is in contrast with the predictions obtained in the diquarkonium picture for the tetraquark model, as found in Section 4. In fact, for a given $J^{P C}$, the model admits both $I=0$ and $I=1$ states. This is for example, the famous case of the charged partners of the $X(3872)$.

From the phenomenological description of the potential it is clear that meson molecules should mainly decay in their constituents. Channels with large phase space are, in fact, mediated by heavier particles, and are therefore suppressed by the large size of the bound state — see again Section 3.2.

As we mentioned at the beginning of the section, the results obtained from the previous potentials are only reliable as long as they are considered as plausibility arguments. There are several instances where the one-pion-exchange model fails. For example, it has been shown $[69,70]$ that the presence of a shallow bound state corresponding to the $X(3872)$ is not certain, as the results strongly depend on the value of the phenomenological cut-offs that one introduces in the regulator of the potential as well as in the vertex form factor. Furthermore [71], since the $X$ is very close to the $D D \pi$ threshold as well (only $\approx 7 \mathrm{MeV}$ ), all the long range terms of the $S$-wave potential are suppressed, leaving only the contact $\delta(r)$ term in the $m_{D^{*}}=m_{D}+m_{\pi}$ limit.

To overcome these problems one can try to go beyond the tree level approximation including, for example, additional terms to describe the short range part of the meson-meson potential or to use a three-body formalism for the $D \bar{D} \pi$ system $[72,73]$. However, these approaches add in general several complications and appear to be rather ad hoc.

\subsubsection{Interaction potential at the quark level}

Because of the small pion mass, the potential described in the previous section is a long range one. An interesting model to describe the short range meson-meson interaction has been introduced in [74], and then applied to the case of the $X(3872)$ in [75] (see also [1]). In this picture, the interaction between the two mesons is described as interaction between their four constituents via the following Hamiltonian

$$
H_{I}=\sum_{i<j} \frac{\lambda_{i}}{2} \cdot \frac{\lambda_{j}}{2}\left(\frac{\alpha_{s}}{r_{i j}}-\frac{3 b}{4} r_{i j}-\frac{8 \pi \alpha_{s}}{3 m_{i} m_{j}} \boldsymbol{S}_{i} \cdot \boldsymbol{S}_{j} \delta^{(3)}\left(\boldsymbol{r}_{i j}\right)\right)
$$

where $\lambda^{a}$ are the Gell-Mann matrices, $\boldsymbol{r}_{i j}$ is the separation between the $i$-th and $j$-th quark, $m_{i}$ their masses and $\boldsymbol{S}_{i}$ their spins. The first and second terms are the phenomenologically well-known Coulomb and confining terms, while the last one is a short range spin-spin hyperfine interaction motivated by one-gluon-exchange. The point-like $\delta$-function is regularized with a Gaussian

$$
\delta^{(3)}\left(\boldsymbol{r}_{i j}\right) \longrightarrow\left(\frac{\sigma^{3}}{\pi^{3 / 2}}\right) e^{-\sigma^{2} r_{i j}^{2}}
$$


The different parameters can be estimated from the observed meson spectrum. It should be mentioned that exponential form factors produce wrong analytic properties in $r^{2}$ and should therefore be avoided.

The interaction in Eq. (3.46) can be used to compute the scattering amplitude for four mesons in a $A B \rightarrow C D$ process [74]. The scattering matrix is parameterized as

$$
S_{A B \rightarrow C D} \equiv-2 \pi i \delta\left(E_{A B}-E_{C D}\right) \delta^{(3)}\left(\boldsymbol{P}_{A B}-\boldsymbol{P}_{C D}\right) h_{A B \rightarrow C D}
$$

For each Feynman diagram describing the four quarks in the $A B \rightarrow C D$ process, the amplitude can be written as

$$
h_{A B \rightarrow C D}^{\text {(diagr.) }}=S I_{\text {flavor }} I_{\text {color }} I_{\text {spin }} I_{\text {space }}
$$

Here $S= \pm 1$ is 'signature' factor that takes into account the permutation of fermion operators in the scattering matrix elements, $I_{\text {flavor }}$ is the overlap coefficient between the flavor states of the incoming and outgoing mesons, $I_{\text {color }}$ is the color factor associated with usual QCD diagrams, and $I_{\text {spin }}$ and $I_{\text {space }}$ are the overlap integrals of the meson spin and space wave functions respectively. See [74] for details.

Once the quark level amplitude $h_{A B \rightarrow C D}$ is known, one can compute the same thing using an effective meson-meson potential. This is usually parametrized as a sum of Gaussians

$$
V_{\mathrm{eff}}=\sum_{i} \alpha_{i} e^{-r^{2} / 2 \beta_{i}^{2}}
$$

Equating the results obtained with the two techniques, the $\alpha_{i}$ and $\beta_{i}$ parameters can be fixed. In Figure 6 we show an example of effective potential extracted from the $D \bar{D}^{*} \rightarrow J / \psi \omega$ process [74].

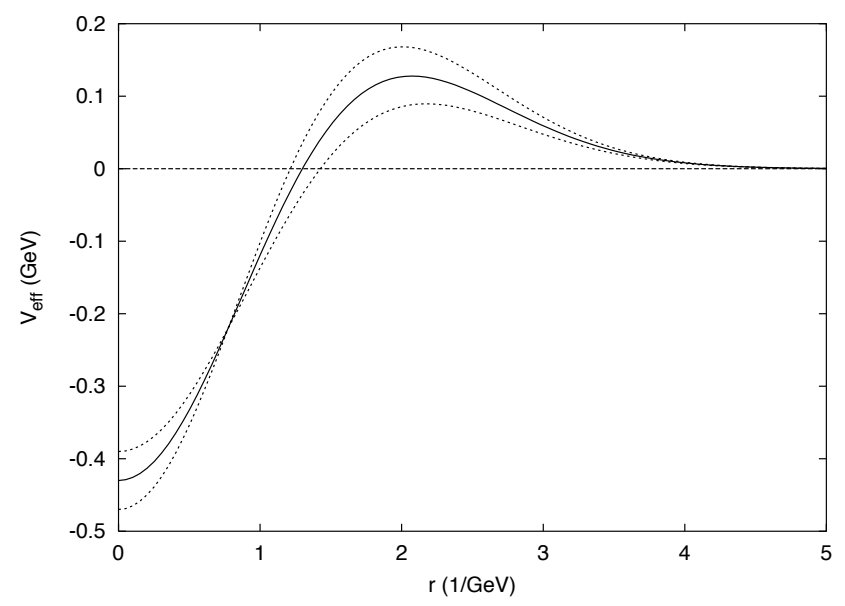

Figure 6: Effective potential for the meson-meson interaction as extracted from the $D \bar{D}^{*} \rightarrow J / \psi \omega$ scattering amplitude. The dashed lines describe the theoretical error due to the approximate knowledge of the initial and final mesons wave function. See [74] for more details.

The shape of the potential shows how this short range interaction could in principle admit bound states in the $D \bar{D}^{*}, J / \psi \omega$ or $J / \psi \rho$ systems. However, given the quark level Hamiltonian (3.46), it is not clear how to distinguish a 'molecular' bound state obtained with it from a compact tetraquark object — see Section 4. The typical distinction between the two cases is that a molecule is an extended object (several fm) while a tetraquark is an almost point-like object. A bound state of $H_{I}$ alone would have the typical hadronic size of $1 \mathrm{fm}$ and hence would be distinguishable from a diquarkonium only because of its color structure. From a quantum field theory point of view, the latter one is a meaningless distinction since different color arrangements mix with each other under renormalization.

In any case, more detailed calculations show that the depth of $V_{\text {eff }}$ is not sufficient, and therefore a contribution from the one-pion-exchange potential needs to be added anyway [75]. On the basis of the observations made in the 
previous section, one can conclude that neither the long range one-pion potential, nor the short range quark potential alone are able to reproduce the observed $X Y Z$ spectrum. If one wants to insist in a molecular description of these states, a combination of the two is needed. However, this inevitably introduces a good degree of model dependence on the physical results.

\subsection{Open problems with the molecular model}

The molecular description of the $X Y Z$ states has many appealing advantages, as we explained during the previous section. However, it is now hopefully clear that it is also plagued by some serious limitations that make its plain application questionable. In summary, the most relevant issues with this framework are

1. It is not able to convincingly solve the prompt production problem. The production cross section of a meson molecule with very small binding energy should be extremely suppressed in high energy collisions at high $p_{\perp}$. The high relative momentum between the two constituents should prevent their binding. This intuition is supported both by MC simulations (see Appendix A) and experimental data on deuteron, Helium-3 and hypertriton (see Section 3.3). This is in striking contrast with the prompt production of the $X(3872)$ with $p_{\perp} \gtrsim 12 \mathrm{GeV}$.

2. The theory of shallow bound states is universal in the limit $E, B \rightarrow 0$ (see Section 2). However, exception made for the $X$, the would-be binding energy of several $X Y Z$ resonances is of some $10 \mathrm{MeV}$. This value escapes the rigorous application of the shallow bound state formalism. If this is not possible, one has to rely on phenomenological realizations of the meson-meson potential (see Section 3.6). This introduces a large degree of model dependence on several quantities like the binding energy, but also on the very existence of bound states. This is clearly not a problem of the molecular interpretation per se, but rather of the available models, which have little predictive power.

3. As it can be seen from Table 2, and its obvious extensions, the molecular model predicts a large number of states which are still largely unobserved.

\section{Diquark building blocks}

Heavy-light diquarks were introduced by Maiani et al. in [7] to discuss the $X(3872)$ following the suggestion of Jaffe and Wilczek [76] to use light diquarks in exotic spectroscopy, with particular reference to some experimental hints of a light pentaquark.

Evidence that in a tetraquark system the two quarks arrange their color in a diquark before interacting with the antiquarks has also been found on the lattice in the static limit [77]. The same authors also show how the four constituents arrange themselves in a $\mathrm{H}$ shaped configuration, as already predicted in the literature [78-82] - see Figure 7.

The diquark-antidiquark model of $X Y Z$ resonances has inspired the search of charged resonances, like the $Z(4430)$ and $Z_{c, b}$ s since it straightforwardly predicts complete charge multiplets, contrarily to the molecular models applied to the description of the $X(3872)$ soon after its discovery.

We are convinced that diquarks are good degrees of freedom to understand the $X Y Z$ phenomenology, and we will illustrate the indisputable successes of the diquark-antidiquark model, together with its obvious limitations. The attempt to incorporate diquarks in an extended picture including the role of meson-meson threshold will be extensively discussed in Section 6. 


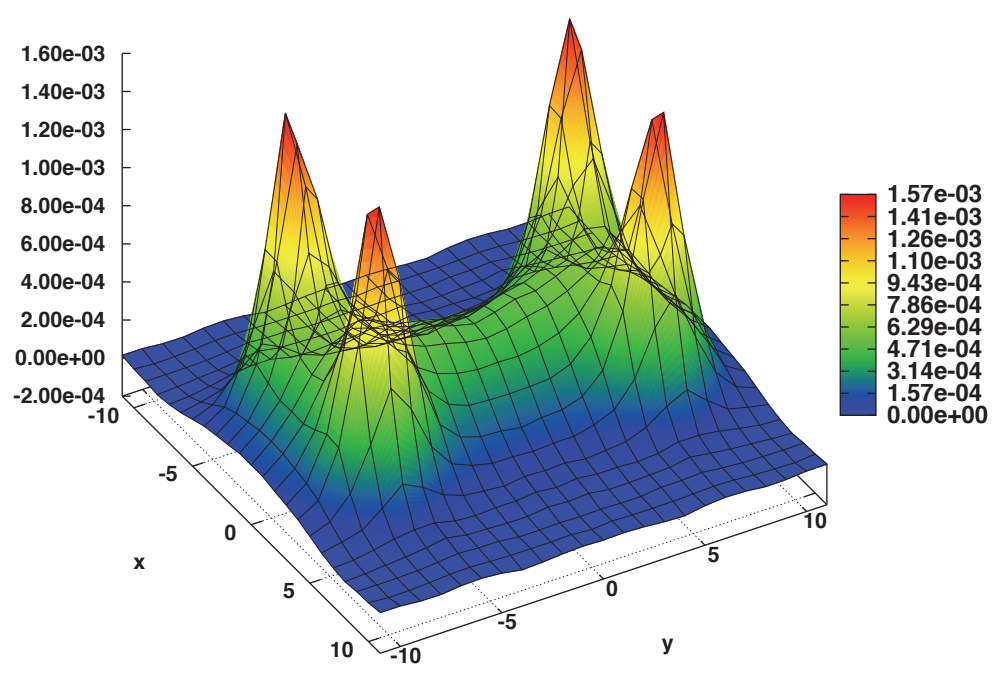

Figure 7: Lagrangian density 3D plot for a four quark system, from [77]. The meson meson mixing with the tetraquark is sufficiently small to produce such a clear tetraquark $\mathrm{H}$ flux tube.

\subsection{General features}

As discussed in Appendix B, in $\mathrm{SU}(3)$ there is attraction between $q q$ pairs in the color antitriplet channel and this is just twice weaker than in the color singlet $q \bar{q}$ in the one-gluon exchange approximation. Because of this we will usually refer to diquarks in the antisymmetric color configuration

$$
d_{\Gamma}^{A}=q^{\alpha} \Gamma q^{\prime \beta}-q^{\beta} \Gamma q^{\prime \alpha} \equiv\left[q q^{\prime}\right]_{S}
$$

where $A$ stands for 'antisymmetric', $\Gamma$ are matrices to characterize the diquark spin $S$ and $\alpha, \beta$ are color indices.

A kind of fermion-boson transformation can be defined

$$
\begin{aligned}
& q \rightarrow \bar{d}^{A} \\
& \bar{q} \rightarrow d^{A}
\end{aligned}
$$

as a generic rule to build new structures with diquarks starting from mesons and baryons.

In particular there is a special relation between baryons and tetraquarks. If we start from an antibaryon, the substitution in Eq. (4.2b) produces the tetraquark $d^{A} \bar{q} \bar{q}=d^{A} \bar{d}^{A}$. Applying (4.2b) once again, one obtains a pentaquark, $d^{A} d^{A} \bar{q}$, and finally, with a third substitution, a state with baryon number $B=2$, a dibaryon with the configuration $d^{A} d^{A} d^{A}$.

It is clear that iterating this procedure a whole bunch of new multiplets of particles might be expected with a large variety of flavors and charges. The first steps in this direction were done in [7, 83]. The search even extended to excited states as discussed [84] and considering also strange light quarks [85]. This program was reported in [2]. As of today the experimental situation in the field of $X Y Z$ resonances does not seem (yet?) to be compatible with such a multitude of particles.

The first and better known resonance, the $X(3872)$, appears as a neutral state alone: the charged counterparts $X^{+}$, obviously predicted by a $d^{A} \bar{d}^{A}$ assignment, have never been observed. Moreover the $X^{0}$ is observed to decay with strong isospin violations, having almost the same branching ratio in the $J / \psi \rho$ and $J / \psi \omega$ decays, as if there were two almost degenerate states $X_{u}=[c u][\bar{c} \bar{u}]$ and $X_{d}=[c d][\bar{c} \bar{d}]$, causing maximal isospin violation. These should be observed in the vicinity of $3872 \mathrm{MeV}$ [86] but, as of today, this has not been the case. 
On the other hand, the diquark approach, differently from all other models of $X Y Z$ states discussed in the literature, was the first pointing to the existence of charged resonances in decays such as $J / \psi \pi^{+}[7,8,87]$. We consider a remarkable success of this model the fact that it stimulated the experimental search, and discovery, of the charged states $Z$ (4430), $Z_{c}(3900), Z_{c}(4020), Z_{b}(10610)$ and $Z_{b}(10650)$. They came as a surprise for those working with molecular models tailored to describe the special case of the $X(3872)$.

The $X(3872)$, besides being the worst enemy against a straightforward construction of tetraquarks as diquarkantidiquark states for the reasons just reminded, has as an additional oddity which has inspired a lot of work: the double-fine-tuning of the $X$ mass with the $J / \psi \rho$ and, especially with $\bar{D}^{0} D^{* 0}$ threshold, which has been discussed at length in previous Sections. The latter case is really remarkable, leaving a difference of few keVs (compatible with zero within experimental errors). To our knowledge there is no simple way to explain this fact in the diquarkonium picture. As we saw in the previous Section, the tiny binding energy of the $X$ can be related to a low energy scattering enhancement in the $\bar{D}^{0} D^{* 0}$ system — with all the difficulties we discussed.

Another straightforward prediction of the diquark-antidiquark model would be the existence of a $[b q][\bar{b} \bar{q}]$ partner in the beauty sector of the $[c q][\bar{c} \bar{q}]$ in the charm one. Also this has not been found so far.

Therefore, despite the success with the qualitative prediction of charged resonances decaying into charmonium + charged meson, the diquark model has way more states than what observed and the research of selection rules within the diquarkonium picture has never been really attempted.

The large number of predicted states is a problem afflicting also models based on the idea of loosely bound molecules. The number of expected states, is basically the same, especially if a resonance is associated to each possible meson-meson threshold. The good point about insisting on the relevance of the vicinity of the $X(3872)$ to the $\bar{D}^{0} D^{* 0}$ threshold is that also the most recently discovered $Z(4430), Z_{c}(3900), Z_{c}(4020), Z_{b}(10610)$ and $Z_{b}(10650)$ have meson-meson thresholds rather close to their masses.

This could also seem to be a casual feature due to the fact that the meson-meson thresholds from the Particle Data Book form a quasi-continuum spectrum of states.

However, looking at things in greater detail, there are very suggestive facts. The $Z_{b}(10610)$ happens to be exactly at the $\bar{B}^{0} B^{* 0}$ threshold, as is the case for the $X(3872) . Z_{c}(3900), Z_{c}(4020)$ and $Z_{b}(10650)$ do not feature the same impressive tuning with thresholds but are still very close to some of them, being a bit heavier — so that no 'standard' molecule interpretation can be given, the binding energy having the wrong sign!

So, on one hand the diquarks seem to be the right tools to easily build a spectroscopy of exotic hadrons like tetraquarks, pentaquarks and dibaryons. On the other hand the feature of vicinity to thresholds seems to have a relevant phenomenological role. In Section 5.3, we will examine the role of diquarks in the $1 / N$ expansion of QCD showing that they are good degrees of freedom to describe tetraquarks in the $s$-channel cuts of meson-meson amplitudes.

In Section 6 we will present a theory which eventually might explain

1. The absence of $X^{+}(3872)$;

2. The absence of a degenerate doublet of $X_{1,2}^{0}$ neutral doublet, required from the naïve diquarkonium picture to explain the isospin violation pattern in the $X$ decay;

3. The extremely small width of the $X(3872)\left(\Gamma_{X} \lesssim 1 \mathrm{MeV}\right)$;

4. The appearance of $Z_{c}$ and of $Z_{b}$;

5. The difference in total widths of these states as related to their mass values.

In order to reach this description of $X Z$ phenomenology, we will begin by reviewing the diquarkonium picture, and then show how diquarkonium discrete levels with some $J^{P C}$ might induce an effective interaction between color neutral mesons with the same quantum numbers, leading to the formation of a resonance [11]. 
A dynamical picture for the diquarkonium has been proposed by Brodsky et al. in [33, 88, 89], which leads to interesting predictions for the decay into charmonia, and for the production cross section at lepton colliders. Different extensions of the constituent quark models for the tetraquark spectroscopy have been explored by Valcarce et al. [90-94], Buccella et al. [95, 96] and Santopinto et al. [97, 98]. The idea of probing the number of constituents by producing tetraquark pairs has been discussed in [33-35]. A different and very interesting viewpoint is given by Rossi and Veneziano [78, 79, 82]. The consequences of color dynamics and Rosner duality for exotic states are reviewed in [99]. Lastly, the problem of light tetraquarks have been approached from a field theoretical point of view using the Dyson-Schwinger formalism in [100]. Its application to the heavy sector as well might give an interesting contribution to the discussion.

In the next few sections, we will review the notion of diquark and especially the algebra needed to deal with them when discussing tetraquarks in the form of diquarkonia.

Diquarks appear in several branches of QCD, especially in the non-perturbative regime, see for example [101] and reference therein, concerning the behavior of hadronic matter at high density studied with techniques of condensed matter theory. New phases of matter and their properties are predicted with interesting consequences also for the astrophysics of neutron stars, for example. Diquarks can have an interesting role also for the discussion of proton and neutron structure functions [102], see also [103]. A tetraquark condensate, built with diquarks, could be the order parameter for an additional chiral phase transition in QCD as recently discussed in [104].

\subsection{Diquarks: color}

In the four spinor formalism the spin zero diquark $J^{P}=0^{+}$is

$$
[c q]_{i}=\epsilon_{i j k} \bar{c}_{c}^{j} \gamma_{5} q^{k} \equiv \epsilon_{i j k}\left(c^{j}\right)^{T} C \gamma_{5} q^{k}
$$


where $i, j$ and $k$ are color indices and $\boldsymbol{c}$ indicates the charge conjugated four-spinor ${ }^{23}$. The charge conjugation operator is

$$
C=i \gamma^{2} \gamma^{0}=\left(\sigma^{2} \otimes i \tau^{2}\right)\left(\mathbb{1} \otimes \tau^{3}\right)=\left(\sigma^{2} \otimes(-1) \tau^{1}\right)=-\left(\begin{array}{cc}
0 & \sigma^{2} \\
\sigma^{2} & 0
\end{array}\right)
$$

where both $\sigma$ and $\tau$ are Pauli matrices. It follows that

$$
C \gamma_{5}=\left(\sigma^{2} \otimes(-1) \tau^{1}\right)\left(\mathbb{1} \otimes \tau^{1}\right)=-\sigma^{2} \otimes \mathbb{1}
$$

Irrespective of the overall phase, we see that (4.3) has a non-relativistic limit, which in the Pauli bispinor notation writes

$$
[c q]_{i}=\epsilon_{i j k}\left(c^{j}\right)^{T} \sigma^{2} q^{k}
$$

In the four spinor formalism the spin one diquark $J^{P}=1^{+}$is

$$
[c q]_{i}=\epsilon_{i j k} \bar{c}_{c}^{j} \gamma q^{k} \equiv \epsilon_{i j k}\left(c^{j}\right)^{T} C \gamma q^{k}
$$

and

$$
C \gamma=-\left(\sigma^{2} \otimes \tau^{1}\right)\left(\sigma \otimes i \tau^{2}\right)=\left(\sigma^{2} \sigma \otimes \tau^{3}\right)
$$

Therefore a spin-1 heavy-light diquark in the non-relativistic limit is

$$
[c q]_{i}^{\lambda}=\epsilon_{i j k}\left(c^{j}\right)^{T} \sigma^{2} \sigma^{\lambda} q^{k}
$$

The formation of diquarks follows from the attractive nature of the color antitriplet $q q$ channel, when color force is mediated by the exchange of one gluon (see also Appendix B). Thus diquarks are antisymmetric in color indices, and they can be antisymmetric in spin, if $S=0$, or symmetric if $S=1$. The fact that we can have both spin 0 and 1 heavy-light diquarks derives from heavy quark spin symmetry.

${ }^{23}$ A light-light diquark with spin 0 has to be antisymmetric in flavor for Fermi statistics. If we consider two flavors only this would write

$$
[q q]_{i}=\epsilon_{i j k} \epsilon^{a b}\left(q^{a j}\right)^{T} C \gamma_{5} q^{b k}
$$

where the flavor structure is (the transposition in (4.4) is with respect to spinor indices)

$$
\epsilon^{a b} q^{a j} q^{b k}=q^{T}\left(i \sigma^{2}\right) q
$$

being $\epsilon^{a b}=\left(i \sigma^{2}\right)_{a b}$ and leaving aside non-flavors labels and matrices commuting with flavor indices.

Under a $\mathrm{SU}(2)$ flavor transformation we have

$$
\left(e^{i \boldsymbol{\sigma} \cdot \boldsymbol{\theta}} q\right)^{T}\left(i \sigma^{2}\right)\left(e^{i \boldsymbol{\sigma} \cdot \boldsymbol{\theta}} q\right)=\left(q^{T} e^{i \boldsymbol{\sigma}^{T} \cdot \boldsymbol{\theta}}\right)\left(i \sigma^{2}\right)\left(e^{i \boldsymbol{\sigma} \cdot \boldsymbol{\theta}} q\right)
$$

Since $\sigma^{2} \sigma=-\sigma^{T} \sigma^{2}$

$$
e^{i \sigma^{T} \cdot \boldsymbol{\theta}}\left(i \sigma^{2}\right) e^{i \boldsymbol{\sigma} \cdot \boldsymbol{\theta}} \equiv i \sigma^{2}
$$

we conclude that (4.4) is SU(2) invariant. With three flavors, the flavor antisymmetric diquark would be

$$
\zeta_{i}^{a}=[q q]_{i}^{a}=\epsilon_{i j k} \epsilon^{a b c}\left(q^{b j}\right)^{T} C \gamma_{5} q^{c k}
$$

which transforms non trivially under SU(3) as

$$
\zeta \rightarrow V \zeta
$$

where

$$
V^{a d}=\frac{1}{2} \epsilon^{a b c} \epsilon^{d b^{\prime} c^{\prime}} U^{b b^{\prime}} U^{c c^{\prime}}
$$

A tetraquark condensate can be constructed

$$
\begin{gathered}
\Phi^{a b}=\left(\zeta_{L i}^{a}\right)^{*} \zeta_{R}^{b j} \\
\Phi \rightarrow V_{R} \Phi V_{L}^{\dagger}
\end{gathered}
$$

i.e. like the standard quark condensate, with interesting consequences for chiral phase transitions [104]. 
The color-spin Hamiltonian describing the interaction between the constituents of a tetraquark is [103]

$$
H=-2 \sum_{i \neq j, a} \kappa_{i j} S_{i} \cdot S_{j} \frac{\lambda_{i}^{a}}{2} \cdot \frac{\lambda_{j}^{a}}{2} \equiv \sum_{i \neq j} H_{i j}
$$

The indices $i, j$ run over the four quarks, while $a$ is the index of the adjoint SU(3) representation. The $\lambda^{a}$ are Gell-Mann matrices, the $S_{i}$ are spin vectors and the $\kappa_{i j}$ are unknown effective couplings. Let us discuss the color interaction for the moment.

We introduce the (normalized) color singlet/octet states using the following notation which turns out to be rather practical for calculations

$$
\begin{aligned}
\left|\bar{c} c_{\mathbf{1}}, \bar{q} q_{\mathbf{1}}\right\rangle & :=\frac{1}{3} \mathbb{1}_{\bar{c} c} \otimes \mathbb{1}_{\bar{q} q} \\
\left|\bar{c} c_{\mathbf{8}}, \bar{q} q_{\mathbf{8}}\right\rangle & :=\frac{1}{4 \sqrt{2}} \lambda_{\bar{c} c}^{a} \otimes \lambda_{\bar{q} q}^{a}
\end{aligned}
$$

where by $\lambda_{\bar{c} c}^{a}$, for example, we mean $\bar{c}_{i}\left(\lambda^{a}\right)_{j}^{i} c^{j}$ using latin letters for color indices.

With the notation $\left|c q_{\overline{\mathbf{3}}}, \bar{c} \bar{q}_{\mathbf{3}}\right\rangle_{\mathbf{1}}$ we mean (aside from overall phases) an overall color singlet state of a diquarkantidiquark pair

$$
\left|c q_{\overline{\mathbf{3}}}, \bar{c} \bar{q}_{\mathbf{3}}\right\rangle_{\mathbf{1}} \sim[c q]_{i}[\bar{c} \bar{q}]^{i}=c_{j} \bar{c}^{j} q_{k} \bar{q}^{k}-c_{j} \bar{q}^{j} q_{k} \bar{c}^{k}
$$

which, using

$$
\left(\lambda^{a}\right)_{j}^{i}\left(\lambda^{a}\right)^{k}=2\left(\delta_{l}^{i} \delta_{j}^{k}-1 / 3 \delta_{j}^{i} \delta_{l}^{k}\right)
$$

can be written as

$$
\left|c q_{\overline{\mathbf{3}}}, \bar{c} \bar{q}_{\mathbf{3}}\right\rangle_{\mathbf{1}} \sim \frac{2}{3} \mathbb{1}_{\bar{c} c} \otimes \mathbb{1}_{\bar{q} q}-\frac{1}{2} \lambda_{\bar{c} c}^{a} \otimes \lambda_{\bar{q} q}^{a}=2\left|\bar{c} c_{\mathbf{1}}, \bar{q} q_{\mathbf{1}}\right\rangle-2 \sqrt{2}\left|\bar{c} c_{\mathbf{8}}, \bar{q} q_{\mathbf{8}}\right\rangle
$$

i.e. the octet-octet component has twice the probability of the singlet-singlet one. This fact may have interesting consequences. Strong interactions are known to preserve the spin of the heavy quark pair (see Section 3.4).

The previous state can itself be normalized in the following way (multiply by $1 / \sqrt{12}$ )

$$
\left|c q_{\overline{\mathbf{3}}}, \bar{c} \bar{q}_{\mathbf{3}}\right\rangle_{\mathbf{1}}=\frac{1}{\sqrt{3}}\left(\frac{1}{3} \mathbb{1}_{\bar{c} c} \otimes \mathbb{1}_{\bar{q} q}-T_{\bar{c} c}^{a} \otimes T_{\bar{q} q}^{a}\right)
$$

and use the $T^{a}=\lambda^{a} / 2$ matrices.

Let us represent states of the fundamental representation with the symbol $\left.\left.\right|_{i}\right\rangle$ whereas those of the anti-fundamental are $\left.\left.\right|^{j}\right\rangle$. Then we have

$$
\begin{aligned}
& \left\langle{ }_{j} \mid T^{a}{ }_{i}\right\rangle=\left(T^{a}\right)^{j}{ }_{i} \\
& \left\langle{ }^{j}\left|T^{a}\right|^{i}\right\rangle=-\left(T^{a}\right)^{i}{ }_{j}
\end{aligned}
$$

i.e. one is the opposite-transposed (complex-conjugate) of the other. From the latter equation we get for example

$$
\left.\left.\left.T^{a}\right|^{i}\right\rangle=-\left.\right|^{j}\right\rangle\left(T^{a}\right)_{j}^{i}
$$

Consider a generic state $|v\rangle$

$$
\left.|v\rangle=\left.\right|^{i}\right\rangle v_{i}
$$

then

$$
\left.\left.\left|T^{a} v\right\rangle=T^{a}|v\rangle=\left.T^{a}\right|^{i}\right\rangle v_{i}=-\left.\right|^{j}\right\rangle\left(T^{a}\right)^{i}{ }_{j} v_{i}
$$




\begin{tabular}{c}
\hline \hline Rules of color manipulation \\
\hline$O_{q_{1}} O_{\bar{q}_{2} q_{1}}^{\prime}=-\left(O O^{\prime}\right)_{\bar{q}_{2} q_{1}} \quad O_{\bar{q}_{2}} O_{\bar{q}_{2} q_{1}}^{\prime}=\left(O O^{\prime}\right)_{\bar{q}_{2} q_{1}}$ \\
$T_{\bar{q}_{1} q_{2}}^{a} \otimes T_{\bar{q}_{3} q_{4}}^{a}=\frac{1}{2} \mathbb{1}_{\bar{q}_{1} q_{4}} \otimes \mathbb{1}_{\bar{q}_{3} q_{2}}-\frac{1}{6} \mathbb{1}_{\bar{q}_{1} q_{2}} \otimes \mathbb{1}_{\bar{q}_{3} q_{4}}$ \\
$\mathbb{1}_{\bar{q}_{1} q_{2}} \otimes \mathbb{1}_{\bar{q}_{2} q_{1}}=\operatorname{Tr}(\mathbb{1})$ \\
$T_{\bar{q}_{1} q_{2}}^{a} \otimes T_{\bar{q}_{2} q_{1}}^{b}=\operatorname{Tr}\left(T^{a} T^{b}\right)$ \\
\hline \hline
\end{tabular}

Table 3: Useful rules for the manipulation of color indices and operators. The quantities with a single quark index are operators acting on the Hilbert space of that quark, e.g. $T_{c}^{a}$ acts on the charm quark, while the quantities with two quark indices are operators saturated with quark states, e.g. $O_{\bar{q}_{1} q_{2}}=\bar{q}_{1 i} O_{j}^{i} q_{2}^{j}$.

Thus we get that (multiply the latter by $\left\langle_{k}\right|$ and then rename $k \rightarrow i$ )

$$
T^{a} v_{i}=-\left(T^{a}\right)^{j}{ }_{i} v_{j}
$$

whereas

$$
T^{a} v^{i}=\left(T^{a}\right)^{i}{ }_{j} v^{j}
$$

It then follows that, aside from an overall minus sign, the different quark indices, e.g $q_{1} q_{2}$ in $T_{q_{1} q_{2}}^{a}$, can be treated as ordinary matrix indices. In Table 3 we report some useful rules for the manipulation of color states and operators. With this rules we can compute the action of of the color part of the $H_{c q}$ Hamiltonian on a diquark state defined in (4.23)

$$
H_{c q}\left|c q_{\overline{\mathbf{3}}}, \bar{c} \bar{q}_{\mathbf{3}}\right\rangle_{\mathbf{1}} \propto T_{c}^{b} T_{q}^{b} \frac{1}{\sqrt{3}}\left(\frac{1}{3} \mathbb{1}_{\bar{c} c} \otimes \mathbb{1}_{\bar{q} q}-T_{\bar{c} c}^{a} \otimes T_{\bar{q} q}^{a}\right)=-\frac{1}{\sqrt{3}}\left(\frac{1}{3} T_{\bar{c} c}^{b} \otimes T_{\bar{q} q}^{b}-\left(T^{a} T^{b}\right)_{\bar{c} c} \otimes\left(T^{a} T^{b}\right)_{\bar{q} q}\right)
$$

and thus

$$
\begin{aligned}
{ }_{\mathbf{1}}\left\langle c q_{\overline{\mathbf{3}}}, \bar{c} \bar{q}_{\mathbf{3}}\left|H_{c q}\right| c q_{\overline{\mathbf{3}}}, \bar{c} \bar{q}_{\mathbf{3}}\right\rangle_{\mathbf{1}} & \propto-\frac{1}{3}\left(\frac{1}{9} \operatorname{Tr}\left(T^{a}\right) \operatorname{Tr}\left(T^{a}\right)-\frac{2}{3} \operatorname{Tr}\left(T^{a} T^{b}\right) \operatorname{Tr}\left(T^{a} T^{b}\right)+\operatorname{Tr}\left(T^{a} T^{b} T^{c}\right) \operatorname{Tr}\left(T^{a} T^{b} T^{c}\right)\right) \\
& =-\frac{1}{3}\left(-2 \frac{2}{3}-\frac{2}{3}\right)=\frac{2}{3}
\end{aligned}
$$

where we have used

$$
\operatorname{Tr}\left(T^{a} T^{b}\right)=\frac{1}{2} \delta^{a b} \quad \text { and } \quad \operatorname{Tr}\left(T^{a} T^{b} T^{c}\right)=\frac{1}{4}\left(d^{a b c}+i f^{a b c}\right)
$$

and

$$
\begin{aligned}
f^{a b c} f^{a b d} & =3 \delta^{a b} \\
d^{a b c} d^{a b d} & =\frac{5}{3} \delta^{a b}
\end{aligned}
$$

This means that, as far as color is concerned, taking matrix elements on diquark-antidiquark color-neutral states, amounts to redefine the chromomagnetic couplings by some representation-dependent numerical factor like $2 / 3$, when the $H_{c q}$ and $H_{\bar{c} \bar{q}}$ terms are considered. Actually we will assume that the dominant couplings in the Hamiltonian are $\kappa_{c q}$ and $\kappa_{\bar{c} \bar{q}}$, i.e., intra-diquark interactions [8]. At a first sight, this assumption may look to violate the $1 / m_{Q}$ scaling of the couplings, which would suggest $\kappa_{q \bar{q}}>\kappa_{c q}>\kappa_{c \bar{c}}$. Instead, this is due to the very short range nature of spin-spin interactions (see also Eq. (3.46) and Sec. 4.4), which makes the interaction between two quarks of different diquarks vanish. This ansatz turns out to be compatible with experimental data. Charge conjugation symmetry also implies $\kappa=\kappa_{c q}=\kappa_{\bar{c} \bar{q}}{ }^{24}$.

\footnotetext{
${ }^{24}$ If extra diquark couplings were considered we could determine them, e.g. $\kappa_{c \bar{c}}$, from the masses of standard $L=0$ mesons observing that ${ }_{\mathbf{1}}\left\langle c q_{\overline{\mathbf{3}}}, \bar{c} \bar{q}_{\mathbf{3}}\left|H_{\bar{c} c}\right| c q_{\overline{\mathbf{3}}}, \bar{c} \bar{q}_{\mathbf{3}}\right\rangle_{\mathbf{1}}=\frac{1}{4}\left\langle\bar{c} c_{\mathbf{1}}, \bar{q} q_{\mathbf{1}}\left|H_{\bar{c} c}\right| \bar{c} c_{\mathbf{1}}, \bar{q} q_{\mathbf{1}}\right\rangle$
} 
In view of what found in this section we understand that mass splittings among tetraquark states conceived as diquarkonia, will be determined solely from the spin-spin part of the Hamiltonian in (4.18).

\subsection{Diquarks: spin}

Heavy-light diquarks are different from light-light ones in that they can have either spin 0 or spin 1, whereas lightlight are preferably spin 0, the so called 'good diquarks' [103]. Heavy quark symmetry is such that the light degrees of freedom are blind to the flavor and spin of the heavy quark source (see again Section 3.4) and this spin decoupling feature makes equally possible heavy $S=0,1$. The use of diquarks in exotic hadron spectroscopy was suggested in [76] and heavy-light diquarks, their properties, and masses were first discussed in [7]. Light-light diquarks and their possible role in light scalar meson spectroscopy is discussed in [105, 106].

We generally use the definitions (see also [4])

$$
\begin{aligned}
\left|1_{\boldsymbol{q}}, 0_{\overline{\boldsymbol{q}}}\right\rangle & =\frac{1}{2} \sigma^{2} \sigma^{i} \otimes \sigma^{2} \\
\left|0_{\boldsymbol{q}}, 1_{\overline{\boldsymbol{q}}}\right\rangle & =\frac{1}{2} \sigma^{2} \otimes \sigma^{2} \sigma^{i} \\
\left|1_{\boldsymbol{q}}, 1_{\overline{\boldsymbol{q}}}\right\rangle_{J=1} & =\frac{i}{2 \sqrt{2}} \epsilon^{i j k} \sigma^{2} \sigma^{j} \otimes \sigma^{2} \sigma^{k}
\end{aligned}
$$

With the symbol $\boldsymbol{q}$ we either mean a diquark in the order $c q$ or $\bar{c} \bar{q}$ or a quark-antiquark pair in the order $c \bar{c}$ or $q \bar{q}$. The ordering is relevant (see [4] for normalizations ${ }^{25}$ ).

In these notations a particle with the quantum numbers of $X(3872)$, would be described by

$$
X=\frac{\left|1_{c q}, 0_{\bar{c} \bar{q}}\right\rangle+\left|0_{c q}, 1_{\bar{c} \bar{q}}\right\rangle}{\sqrt{2}}=\left|1_{c \bar{c}}, 1_{q \bar{q}}\right\rangle_{J=1}=\frac{\left|1_{c \bar{q}}, 0_{q \bar{c}}\right\rangle-\left|0_{c \bar{q}}, 1_{q \bar{c}}\right\rangle}{\sqrt{2}}
$$

Since diquarks are defined to be positive parity states, overall we have $J^{P}=1^{+}$and $C=+$. This diquark-antidiquark arrangement is a natural candidate to describe the $X(3872)$, which is a $1^{++}$resonance decaying into $J / \psi+\rho / \omega$, compatibly with the $\left|1_{c \bar{c}}, 1_{q \bar{q}}\right\rangle_{J=1}$ assignment — especially for what concerns the heavy spin. Similarly the last term on the rhs is compatible with the $D D^{*}$ decay mode of the $X(3872)$. Anyway, light quark spins in $Q \bar{q}$ or $\bar{Q} q$ configurations might rearrange also to allow $D D$ or $D^{*} D^{*}$ decays but the latter is phase space forbidden and the former is simply forbidden by quantum numbers.

The orthogonal combination to the lhs of (4.37) can be formed by

$$
Z=\frac{\left|1_{c q}, 0_{\bar{c} \bar{q}}\right\rangle-\left|0_{c q}, 1_{\bar{c} \bar{q}}\right\rangle}{\sqrt{2}}=\frac{\left|1_{c \bar{c}}, 0_{q \bar{q}}\right\rangle-\left|0_{c \bar{c}}, 1_{q \bar{q}}\right\rangle}{\sqrt{2}}=\left|1_{c \bar{q}}, 1_{q \bar{c}}\right\rangle_{J=1}
$$

This has $J^{P}=1^{+}$and $C=-$ for the neutral component (if an isospin triplet is to be considered, the $G$-parity has to be $G=+)$. The state has $C=-$ since, in the charmonium basis

$$
C=(-1)^{L+S_{q \bar{q}}+S_{c \bar{c}}}
$$

\footnotetext{
${ }^{25}$ For example, the normalization in $(4.35 \mathrm{c})$ is obtained by

$$
\left\langle 1_{\boldsymbol{q}}, 1_{\overline{\boldsymbol{q}}} \mid 1_{\boldsymbol{q}}, 1_{\overline{\boldsymbol{q}}}\right\rangle_{J=1}=-\frac{1}{4 \times 2}\left(\operatorname{Tr}\left(\sigma^{j T} \sigma^{j}\right) \operatorname{Tr}\left(\sigma^{k T} \sigma^{k}\right)-\operatorname{Tr}\left(\sigma^{j T} \sigma^{k}\right) \operatorname{Tr}\left(\sigma^{k T} \sigma^{j}\right)\right)=-\frac{1}{4 \times 2}(2 \times 2-2 \times 2 \times 3)=1
$$
}


In the quark-antiquark basis there is another state with $C=-$, orthogonal to $Z$

$$
Z^{\prime}=\left|1_{c q}, 1_{\bar{c} \bar{q}}\right\rangle_{J=1}=\frac{\left|1_{c \bar{c}}, 0_{q \bar{q}}\right\rangle+\left|0_{c \bar{c}}, 1_{q \bar{q}}\right\rangle}{\sqrt{2}}=\frac{\left|1_{c \bar{q}}, 0_{q \bar{c}}\right\rangle+\left|0_{c \bar{q}}, 1_{q \bar{c}}\right\rangle}{\sqrt{2}}
$$

which is indeed a $1^{+-}$state. Exchanging the coordinates, spins and charges of two fermions/bosons having each spin $s$ and total spin $S$, the total wavefunction has to be completely antisymmetric/symmetric under this exchange

$$
(-1)^{L}(-1)^{2 s+S} C=\mp 1
$$

which in the case of (4.40) (first term on the rhs) is

$$
(-1)^{0}(-1)^{2+1} C=+1
$$

giving $C=-$. The case of $X=\left|1_{c \bar{c}}, 1_{q \bar{q}}\right\rangle_{J=1}$ is different as the charge conjugation operator concerns the distinct $c \bar{c}$ and $q \bar{q}$ pairs.

Linear combinations of $Z$ and $Z^{\prime}$ which diagonalize the spin-spin Hamiltonian can be identified with $Z_{c}(3900)$ and $Z_{c}(4020)$. We will not treat here scalar or tensor states whose discussion in terms of diquarkonia can $b$ found in [4].

\subsection{Spectrum for $L=0$ and the $X, Z, Z^{\prime}$ system}

We assume that the spin-spin interactions within the diquark shells are dominant with respect to quark-antiquark interactions. This is the main assumption in [8] and, as we will see, it gives the right mass pattern for the $X, Z_{c}, Z_{c}^{\prime}$ system.

This hypothesis leads to believe that diquarks are quite separated from each other in the tetraquark and this feature could be modeled with some potential keeping the diquarks relatively apart in space — recall that the spin-spin interaction is proportional to $\delta(r)$ as in Eq. (3.46). At the same time this potential might be responsible for the reluctance of diquarks at mixing or decaying into meson pairs. Widths of actual diquarkonia measure this behavior.

Then spin-spin Hamiltonian is (color factors have been reabsorbed in the definition of chromomagnetic couplings as discussed above)

$$
H \approx 2 \kappa\left(\boldsymbol{S}_{q} \cdot \boldsymbol{S}_{c}+\boldsymbol{S}_{\bar{q}} \cdot \boldsymbol{S}_{\bar{c}}\right)
$$

Consider for example

$$
4 \boldsymbol{S}_{q} \cdot \boldsymbol{S}_{c}\left|1_{c q}, 0_{\bar{c} \bar{q}}\right\rangle=\sigma_{(q)} \cdot \boldsymbol{\sigma}_{(c)}\left|1_{c q}, 0_{\bar{c} \bar{q}}\right\rangle:=\frac{1}{2}\left(\sigma^{j}\right)^{T} \sigma^{2} \sigma^{i} \sigma^{j} \otimes \sigma^{2}
$$

where summation over $j$ is understood. The matrix $\left(\sigma^{j}\right)^{T}$ works on $c$ whereas $\sigma^{j}$ on $q$. Considering that

$$
\frac{1}{2}\left(\sigma^{j}\right)^{T} \sigma^{2} \sigma^{i} \sigma^{j} \otimes \sigma^{2}=-\frac{1}{2}\left(\sigma^{2} \sigma^{j} \sigma^{i} \sigma^{j}\right) \otimes \sigma^{2}=\frac{1}{2} \sigma^{2} \sigma^{i} \otimes \sigma^{2}=\left|1_{c q}, 0_{\bar{c} \bar{q}}\right\rangle
$$

where we have used $i \epsilon^{i j k} \sigma^{j} \sigma^{k}=i \epsilon^{i j k} i \epsilon^{j k \ell} \sigma^{\ell}=-2 \sigma^{i}$. Thus $\left|1_{c q}, 0_{\bar{c} \bar{q}}\right\rangle$ is an eigenstate of $\boldsymbol{S}_{q} \cdot \boldsymbol{S}_{c}$ with eigenvalue $1 / 4$.

Considering also the antidiquark contribution one readily finds

$$
4 S_{\bar{q}} \cdot S_{\bar{c}}\left|1_{c q}, 0_{\bar{c} \bar{q}}\right\rangle=-3\left|1_{c q}, 0_{\bar{c} \bar{q}}\right\rangle
$$

thus

$$
4\left(\boldsymbol{S}_{q} \cdot \boldsymbol{S}_{c}+\boldsymbol{S}_{\bar{q}} \cdot \boldsymbol{S}_{\bar{c}}\right)\left|1_{c q}, 0_{\bar{c} \bar{q}}\right\rangle=-2\left|1_{c q}, 0_{\bar{c} \bar{q}}\right\rangle
$$

and

$$
H\left|1_{c q}, 0_{\bar{c} \bar{q}}\right\rangle=-\kappa\left|1_{c q}, 0_{\bar{c} \bar{q}}\right\rangle
$$


Similarly

$$
\begin{aligned}
H\left|0_{c q}, 1_{\bar{c} \bar{q}}\right\rangle & =-\kappa\left|0_{c q}, 1_{\bar{c} \bar{q}}\right\rangle \\
H\left|1_{c q}, 1_{\bar{c} \bar{q}}\right\rangle_{J=1} & =+\kappa\left|1_{c q}, 1_{\bar{c} \bar{q}}\right\rangle_{J=1}
\end{aligned}
$$

The Hamiltonian (4.43) is diagonal in the diquark-antidiquark basis formed by the $1^{+-}$states

$$
(H)^{1^{+-}}=\left(\begin{array}{cc}
-\kappa & 0 \\
0 & \kappa
\end{array}\right)
$$

with eigenvectors $|1\rangle=1 / \sqrt{2}(|1,0\rangle-|0,1\rangle) \equiv|Z\rangle$ and $|2\rangle=|1,1\rangle_{J=1} \equiv\left|Z^{\prime}\right\rangle$. This requires $|Z\rangle$ to be lighter than $\left|Z^{\prime}\right\rangle$. Similarly

$$
(H)^{1^{++}}=-\kappa
$$

Therefore we conclude that the $X(3872)$ and the $Z(3900)$ are degenerate in first approximation, their masses being twice the diquark mass plus the same spin-spin interaction correction

$$
M_{X}=M_{Z}=2 m_{[c q]}-\kappa
$$

The $Z^{\prime}$ is instead heavier by a gap of $2 \kappa$

$$
M\left(Z^{\prime}\right)=2 m_{[c q]}+\kappa
$$

This pattern of $X, Z, Z^{\prime}$ masses is observed in experimental data. In particular, the two relations in Eqs. (4.52) and (4.53) are both satisfied within $20 \mathrm{MeV}$ for

$$
\kappa=67 \mathrm{MeV}
$$

\subsection{Scalar and tensor states}

The diquark-antidiquark model also allows $J^{P}=0^{+}, 2^{+}$states with $C=+$. We have two $J^{P}=0^{+}$states and a tensor one

$$
\begin{aligned}
& X_{0}=\left|0_{c q}, 0_{\bar{c} \bar{q}}\right\rangle_{J=0}=\frac{1}{2}\left|0_{c \bar{c}}, 0_{q \bar{q}}\right\rangle-\frac{\sqrt{3}}{2}\left|1_{c \bar{c}}, 1_{q \bar{q}}\right\rangle_{J=0} \\
& \left.X_{0}^{\prime}=\left|1_{c q}, 1_{\bar{c} \bar{q}\rangle_{J=0}}=\frac{\sqrt{3}}{2}\right| 0_{c \bar{c}}, 0_{q \bar{q}}\right\rangle+\frac{1}{2}\left|1_{c \bar{c}}, 1_{q \bar{q}}\right\rangle_{J=0} \\
& X_{2}=\left|1_{c q}, 1_{\bar{c} \bar{q}}\right\rangle_{J=2}=\left|1_{c \bar{c}}, 1_{q \bar{q}}\right\rangle_{J=2}
\end{aligned}
$$

which are all charge-conjugation even, $C=+$. Following the same notations introduced above we have

$$
\begin{aligned}
\left|0_{\boldsymbol{q}}, 0_{\boldsymbol{q}}\right\rangle_{J \equiv 0} & =\frac{1}{2} \sigma^{2} \otimes \sigma^{2} \\
\left|1_{\boldsymbol{q}}, 1_{\boldsymbol{q}}\right\rangle_{J=0} & =\frac{1}{2 \sqrt{3}} \sigma^{2} \sigma^{i} \otimes \sigma^{2} \sigma^{i} \\
\left|1_{\boldsymbol{q}}, 1_{\boldsymbol{q}}\right\rangle_{J=2} & =\frac{1}{2}\left(\sigma^{2} \sigma^{(i} \otimes \sigma^{2} \sigma^{j)}-\frac{2}{3} \delta^{i j} \sigma^{2} \sigma^{\ell} \otimes \sigma^{2} \sigma^{\ell}\right)
\end{aligned}
$$

where $i, j$ indices are symmetrized in the last equation (a factor of $1 / 2$ has to be included in the symmetrization) and the trace is subtracted. For the determination of the normalizations coefficients see [4]. 
We can also determine

$$
\begin{aligned}
& (H)^{0^{++}}=-3 \kappa \\
& (H)^{0^{++}}=\kappa \\
& (H)^{2^{++}}=\kappa
\end{aligned}
$$

There are no official candidates for these scalar and tensor states. However, there is the tantalizing possibility for the $Z_{1}$ (4050) state decaying into $\chi_{c 1} \pi$ to be a scalar or a tensor (see for example [107]). In this case, it would be degenerate with the $Z_{c}^{\prime}(4020)$ within errors, and would be the natural candidate for the $0^{++\prime}\left(2^{++}\right)$tetraquark. We also remark that, if the $Z(4430)$ is considered as the radial excitation of the $Z_{c}(3900)$, it is natural to identify the $Z_{2}(4250)$ as the radial excitation of the lowest $0^{++}$tetraquark, given that their mass difference, $m_{Z(4430)}-m_{Z_{1}(4250)} \simeq(230 \pm 190) \mathrm{MeV}$ is compatible with $2 \kappa \simeq 130 \mathrm{MeV}$.

In Figure 8 we show a pictorial representation the spectrum obtained from the Hamiltonian (4.43). In Table 4 we instead list the predicted states and their quantum numbers.

$$
\begin{array}{ll}
\frac{0^{++^{\prime}}}{+\kappa} & \frac{1^{+-^{\prime}}}{}+\kappa \quad \frac{2^{++}}{}+\kappa \\
\frac{1^{++}}{-\kappa} \quad \frac{1^{+-}}{}-\kappa \\
\frac{0^{++}-3 \kappa}{}
\end{array}
$$

Figure 8: The spectrum of Hamiltonian in Eq. (4.43).

Table 4: Summary table of states. We refer here to the neutral components.

\begin{tabular}{lll}
\hline \hline$J^{P C}$ & $\left|S_{c q}, S_{\bar{c} \bar{q}}\right\rangle$ & $\left|S_{c \bar{c}}, S_{q \bar{q}}\right\rangle$ \\
\hline $0^{++}$ & $|0,0\rangle$ & $\frac{1}{2}|0,0\rangle+\frac{\sqrt{3}}{2}|1,1\rangle_{J=0}$ \\
$0^{++}$ & $|1,1\rangle_{J=0}$ & $\frac{\sqrt{3}}{2}|0,0\rangle-\frac{1}{2}|1,1\rangle_{J=0}$ \\
$1^{++}$ & $\frac{1}{\sqrt{2}}(|1,0\rangle+|0,1\rangle)$ & $|1,1\rangle_{J=1}$ \\
$1^{+-}$ & $\frac{1}{\sqrt{2}}(|1,0\rangle-|0,1\rangle)$ & $\frac{1}{\sqrt{2}}(|1,0\rangle-|0,1\rangle)$ \\
$1^{+-}$ & $|1,1\rangle_{J=1}$ & $\frac{1}{\sqrt{2}}(|1,0\rangle+|0,1\rangle)$ \\
$2^{++}$ & $|1,1\rangle_{J=2}$ & $|1,1\rangle_{J=2}$ \\
\hline \hline
\end{tabular}

\section{6. $Y$ resonances and $L=1$ diquarkonia}

Tetraquarks with $J^{P C}=1^{--}$can be obtained with odd values of the angular momentum. The lowest lying have $L=1$. We select states with odd charge conjugation. 
In the diquark-antidiquark basis of $[c q][\bar{c} \bar{q}]$ we have

$$
\begin{array}{ll}
Y_{1}=\left|0_{c q}, 0_{\bar{c} \bar{q}}\right\rangle & C=(-1)^{L=1} \\
Y_{2}=\frac{\left|1_{c q}, 0_{\bar{c} \bar{q}}\right\rangle+\left|0_{c q}, 1_{\bar{c} \bar{q}}\right\rangle}{\sqrt{2}} & C=(-1)^{L=1} \\
Y_{3}=\left|1_{c q}, 1_{\bar{q} \bar{q}}\right\rangle_{S=0} & (-1)^{L}(-1)^{2 s+S} C=+1 \Rightarrow C=(-1)^{1}(-1)^{2 \times 1+0} \\
Y_{4}=\left|1_{c q}, 1_{\bar{c} \bar{q}}\right\rangle_{S=2} & C=(-1)^{1}(-1)^{2 \times 1+2}
\end{array}
$$

The spin structure of $Y_{2}$ and $X$ in (4.37) is the same and therefore the mass difference between them might entirely be attributed to the orbital excitation of $Y_{2}$. The fact that $Y_{2}$ and $X$ have the same spin structure also suggests that radiative transitions with $\Delta L=1$ and $\Delta S_{c \bar{c}}=0$ might occur

$$
Y_{2} \rightarrow \gamma X
$$

As an important caveat, we have to add here that the experimental assessment of the $Y$ states, at the time of this writing, is still rather unclear, even more so with the new results by BES III [108, 109]. In particular, the very existence of a $Y(4008)$ is controversial. The identification of the structures seen in $J / \psi \pi \pi$ with the ones in $h_{c} \pi \pi$ is problematic too, and would undermine the assignment even of the well known $Y(4260)$ (see Section 8.4 for the experimental details). The assignments made below are based on the existence of these resonance, and are therefore subject to variation if these state turn out not to be confirmed. We preferred to stick to these hypotheses for the sake of illustration of the method of constructing the states in the diquarkonium picture. As we will comment in Section 6, orbitally excited and radially excited resonances present more difficulties than lowest lying states.

Anyway, if a distinct $Y(4260)$ does exist, its identification as the $Y_{2}$ excitation would be supported by the conspicuous radiative decay mode [110]

$$
Y(4260) \rightarrow X(3872)+\gamma
$$

The $Y(4360)$ and $Y(4660)$ are interpreted as radial excitations of $Y_{1}=Y(4008)$ (see Table 5) and $Y_{2}=Y(4260)$. As a check for this assumption we may note that they indeed present mass splitting similar to the one observed between ordinary ground state and excited charmonia, i.e. $M\left(\chi_{b J}(2 P)\right)-M\left(\chi_{b J}(1 P)\right) \simeq M(Y(4360))-M(Y(4008))$ and $M\left(\chi_{c J}(2 P)\right)-M\left(\chi_{c J}(1 P)\right) \simeq M(Y(4660))-M(Y(4260))$. For the identification of the $Y_{3}$ state as the structures seen in $e^{+} e^{-} \rightarrow h_{c} \pi \pi, \chi_{c 0} \omega$, see [111].

As for the $Y(4630)$, decaying predominantly into $\Lambda_{c}^{+} \Lambda_{c}^{-}$, we recall that there is also the possibility of its assignment to a tetraquark degenerate with $Y(4660)$ [81].

Table 5: Possible assignments for $Y_{1}, Y_{2}, Y_{3}, Y_{4}$. Observe that $Y_{3}$ is predicted to decay preferably in $h_{c}(1 P)$ where $S_{c \bar{c}}=0$. The state $Y(4220)$ correspond to the narrow structure described in $[109,112,113]$. Radiative decays are suggested by conservation of the heavy quark spin $\Delta S_{c \bar{c}}=0$. The relative probability of having spin 1 versus spin 0 in the $c \bar{c}$ pair as read by Table 4 .

\begin{tabular}{cccc}
\hline \hline State & $P\left(S_{c \bar{c}}=1\right): P\left(S_{c \bar{c}}=0\right)$ & Assignment & Radiative Decay \\
\hline$Y_{1}$ & $3: 1$ & $Y(4008)$ & $\gamma+X\left(0^{++}\right)$ \\
$Y_{2}$ & $1: 0$ & $Y(4260)$ & $\gamma+X\left(1^{++}\right)$ \\
$Y_{3}$ & $1: 3$ & $Y(4220)$ & $\gamma+X\left(0^{++}\right)$ \\
$Y_{4}$ & $1: 0$ & $Y(4630)$ & $\gamma+X\left(2^{++}\right)$ \\
\hline \hline
\end{tabular}

To find the spectrum of the $Y$ states we use the same Hamiltonian form (4.43) with the addition of a spin-orbit and a purely orbital term - here the chromomagnetic coupling $\kappa^{\prime}$ is taken to be a priori different from $\kappa$ used in (4.43). 
We already discussed that the spatial separation of the diquarks makes the other couplings vanish. We have then

$$
H \approx 2 \kappa^{\prime}\left(\boldsymbol{S}_{q} \cdot \boldsymbol{S}_{c}+\boldsymbol{S}_{\bar{q}} \cdot \boldsymbol{S}_{\bar{c}}\right)-2 A \boldsymbol{S} \cdot \boldsymbol{L}+\frac{1}{2} B \boldsymbol{L}^{2}
$$

in such a way that the energy increases for increasing $L^{2}$ and $S^{2}$, provided $\kappa^{\prime}, A, B$ are positive. Indeed the masses of $Y$ states will be given by

$$
M=M_{0}^{\prime}+\kappa^{\prime}(s(s+1)+\bar{s}(\bar{s}+1)-3)+A(L(L+1)+S(S+1)-2)+B \frac{L(L+1)}{2}
$$

where $s, \bar{s}$ are the total spins of diquark and antidiquark. The latter equation can be simplified to

$$
M=M_{0}+(A+B / 2) L(L+1)+A S(S+1)+\kappa^{\prime}(s(s+1)+\bar{s}(\bar{s}+1))
$$

with

$$
M_{0}=M_{0}^{\prime}-2 A-3 \kappa^{\prime}
$$

From Eq. (4.63), the mass of the state $Y_{1}$ in (4.58a) is given by

$$
M_{1}=M_{0}+2(A+B / 2)
$$

for $s=\bar{s}=0$, therefore implying $S=0$, and $L=1$. The $Y_{2}$ state in (4.58b) has $s=1$ or $\bar{s}=1$, thus $S=1-$ considering that $M_{0}$ contains $-3 \kappa^{\prime}$ we can determine the mass gap between $Y_{2}$ and $Y_{1}$

$$
M_{2}-M_{1}=2 \kappa^{\prime}+2 A
$$

hence implying $M_{2}>M_{1}$. The $Y_{3}$ state has both spins $s=\bar{s}=1$ but $S=0$ so that

$$
M_{3}-M_{2}=2 \kappa^{\prime}-2 A
$$

which can take either sign depending on the $\kappa^{\prime}-A$ difference; $\kappa^{\prime}$ and $A$ have in principle similar sizes. Finally $Y_{4}$ has both spins $s=\bar{s}=1$ and $S=2$ so that

$$
M_{4}-M_{3}=6 A
$$

implying $M_{4}>M_{3}$. So the mass ordering is $M_{1}, M_{2}, M_{3}, M_{4}$ or $M_{1}, M_{3}, M_{2}, M_{4}$ from lighter to heavier. Using the assignments in Table 5, from (4.66) and (4.67) we obtain ${ }^{26}$ :

$$
\kappa^{\prime}=53 \mathrm{MeV} \quad \text { and } \quad A=73 \mathrm{MeV}
$$

The values found for $\kappa^{\prime}$ have to be compared with the value of $\kappa=67 \mathrm{MeV}$ obtained studying the spectrum of $L=0$ states. They look reasonably consistent, also in consideration of the simplicity of the model described. In the original formulation for the diquarkonium model for the $X Y Z$ states, all the couplings $\kappa_{i j}$ were kept non-zero and their value was fitted from known meson and baryon masses. In that case is was found $\kappa \simeq 22 \mathrm{MeV}$. We conclude that diquarks inside a tetraquark behave differently than those inside a baryon.

\subsection{Pentaquarks from diquarks}

In the diquark composition model, pentaquarks are clusters of diquarks of the form

$$
P=d^{A} d^{A} \bar{q}
$$

\footnotetext{
${ }^{26}$ In the original paper [8] also the possible assignment $Y_{3}=Y(4290)$ was considered. However, the energy scan by BES [109] pushes the mass of the state to be $100 \mathrm{MeV}$ heavier, thus making this assignment unlikely.
} 
where $d^{A}$ is the antisymmetric diquark introduced in Eq. (4.1). There are several flavor compositions which may realize a pentaquark, and the diquark model would clearly predict a great variety of states.

Eventually there was a stunning observation by the LHCb collaboration [114] reporting the observation of two new resonances in the $\Lambda_{b}$ decay

$$
\Lambda_{b}(b u d) \rightarrow P^{+} K^{-}
$$

each decaying according to

$$
P^{+} \rightarrow J / \psi p
$$

Thus the new particles carry a unit of baryonic number and feature the valence quark composition

$$
P^{+}=\bar{c} \text { cuud }
$$

whence the name pentaquarks. The best fit quantum numbers and masses are

$$
\begin{array}{lll}
J^{P}=3 / 2^{-} & M \simeq 4380 \mathrm{GeV} & \text { fract. } \simeq 8.4 \% \\
J^{P}=5 / 2^{+} & M \simeq 4450 \mathrm{GeV} & \text { fract. } \simeq 4.1 \%
\end{array}
$$

The very fact that two pentaquarks have been observed with opposite parities strongly suggests that diquarks should have a role in their composition - bound states of color neutral hadrons cannot appear in orbitally excited configurations.

The quarks in (4.73) have to be arranged into diquarks of different spin and flavor. The standard lore that lightlight diquarks have to be spin zero, which has some support from lattice calculations [115], is dubious in the case of diquarks in baryons. Indeed spin one light-light diquarks (Jaffe's 'bad diquarks'), while conspicuously absent in light meson spectroscopy, are well established in baryons as indicated by the $\Sigma-\Lambda$ mass difference [116] and confirmed by $\Sigma_{c, b}-\Lambda_{c, b}$ mass differences [7].

At first sight, the near $70 \mathrm{MeV}$ difference between the masses in (4.74) does not go well with the energy associated to orbital excitation. One orbital excitation in mesons and baryons carries an energy difference which is typically of order $300 \mathrm{MeV}$, as exemplified by the mass difference $M(\Lambda(1405))-M(\Lambda(1116)) \simeq 290 \mathrm{MeV}$. Mass formulae for the orbital excitation in $X Y Z$ mesons are discussed in [8] and the associated energy difference is estimated to be $\Delta M(L=0 \rightarrow 1) \simeq 280 \mathrm{MeV}$.

However, the mass difference between light-light diquarks with spin $s=1,0$ [116], estimated from charm and beauty baryon spectra, is of order $200 \mathrm{MeV}$, e.g. $M\left(\Sigma_{c}(2455)\right)-M\left(\Lambda_{c}(2286)\right) \simeq 170 \mathrm{MeV}$ and $M\left(\Sigma_{b}(5811)\right)-$ $M\left(\Lambda_{b}(5620)\right) \simeq 190 \mathrm{MeV}$.

If we use spin one light-light diquarks in the compositions

$$
\begin{aligned}
& P\left(3 / 2^{-}\right)=\left(\bar{c}[c q]_{s=1}\left[q^{\prime} q^{\prime \prime}\right]_{s=1}, L=0\right) \\
& P\left(5 / 2^{+}\right)=\left(\bar{c}[c q]_{s=1}\left[q^{\prime} q^{\prime \prime}\right]_{s=0}, L=1\right)
\end{aligned}
$$

the orbital gap is reduced to about $100 \mathrm{MeV}$, which brings it back to the range of spin-spin and spin-orbit corrections indicated by (4.74). In other works the heavier diquark in the $L=0$ state shortens the mass distance from the $L=1$ state with a spin zero (i.e. lighter) diquark.

Notice that the intrinsic parity of the $L=0$ state is dictated by the presence of an antiquark: therefore, differently from standard baryons, the low lying state has negative parity. The opposite holds true for tetraquarks: the low lying ones have positive parities - whereas the low-lying mesons have negative parities.

Concerning the production of a spin-1, light-light $[u d]$ diquark in $\Lambda_{b}$ decay, we note that there are in fact two possible mechanisms leading to the pentaquark production — see Figure 9. In the first one (diagram $A$ in Figure 9) the 


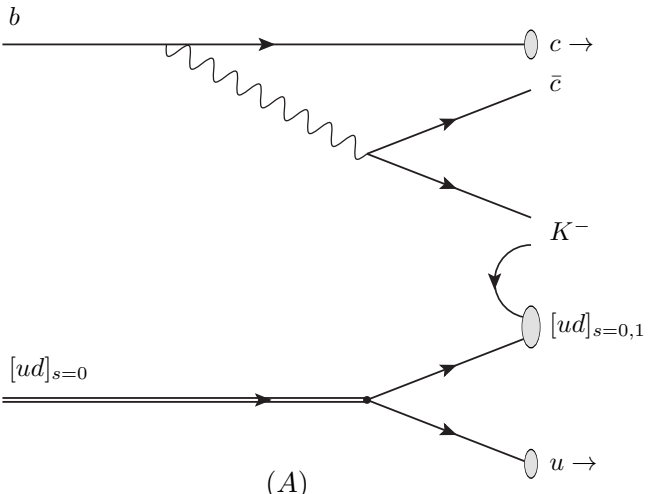

(A)

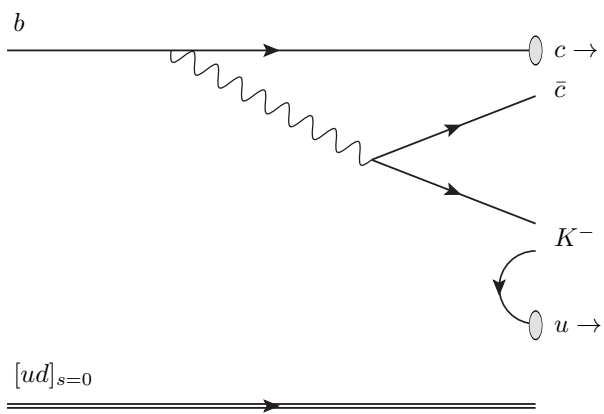

(B)

Figure 9: $(A)$ : The $[u d]$, spin zero diquark in the $\Lambda_{b}$ is transmitted to the $P_{u}$ type pentaquark as a spectator - it is expected that $\Lambda_{b}$ contains a spin zero light-light diquark of the $[u d]$ flavor. $(B)$ : The $u$ quark from the vacuum participates in the formation of the light-light diquark: spin zero and one are both permitted. Mechanism (B) may also produce a $[u u]_{s=1}$ diquark.

$b$-quark spin is shared between the Kaon and the $\bar{c}$ and $[c u]$ components. Barring angular momentum transfer due to gluon exchanges between the light diquark and light quarks from the vacuum, the final $[u d]$ diquarks has to have spin zero. In the second mechanism, however (diagram $B$ ), the $[u d]$ diquark is formed from the original $d$ quark and the $u$ quark from the vacuum. Angular momentum is shared among all final components and the $[u d]$ diquark may well have spin one.

Diquarks are antisymmetric in color indices and can be symmetric or antisymmetric in spin indices $(S=1,0$ respectively). When a diquark contains light quarks with flavors $u, d, s$, the overall state must be antisymmetric by Fermi statistics (strong interactions do not distinguish the flavors $u, d, s$ ). That means

$$
\begin{aligned}
& S=0 \Rightarrow \text { Flavor Antisymmetric } \\
& S=1 \Rightarrow \text { Flavor Symmetric }
\end{aligned}
$$

A diquark with the composition $[u u],[d d]$ or $[s s]$ must be spin 1 necessarily. Pentaquarks realizing the valence quark structure (4.73) are therefore of two types

$$
\begin{aligned}
P_{u} & =\epsilon^{i j k} \bar{c}_{i}[c u]_{j, S=0,1}[u d]_{k, S=0,1} \\
P_{d} & =\epsilon^{i j k} \bar{c}_{i}[c d]_{j, S=0,1}[u u]_{k, S=1}
\end{aligned}
$$

where greek indices are for color, diquarks are in the color antisymmetric, $\overline{\mathbf{3}}$, configuration and overall antisymmetry requires flavor symmetric light-light diquark with $s=1$. The $P_{d}$ component can appear only in (4.75).

This shows that a remarkable number of pentaquarks are immediately predicted once an SU(3) analysis is done. Depending on the flavor symmetry of the light-light quark, given that the heavy-light has flavor $\mathbf{3}$, we have that, depending on the spin of the light-light diquark

$$
\begin{aligned}
& S=0 \Rightarrow \mathbf{3} \otimes \overline{\mathbf{3}}=\mathbf{1} \oplus \mathbf{8} \\
& S=1 \Rightarrow \mathbf{3} \otimes \mathbf{6}=\mathbf{8} \oplus \mathbf{1 0}
\end{aligned}
$$

Moreover the $J$ quantum numbers have to be considered. If we stick to the case $L=0$, depending on the spin $S$ of the light-light diquark we have

$$
\begin{aligned}
& S=0 \Rightarrow J=1 / 2(2 \text { ways }), J=3 / 2(1 \text { way }) \\
& S=1 \Rightarrow J=1 / 2(3 \text { ways }), J=3 / 2 \text { ( } 3 \text { ways }), J=5 / 2(1 \text { way })
\end{aligned}
$$


which gives a further $J$ multiplicity of 10 states. An analysis of expected states and decays in the diquark model can be found in [117] together with an extension in [118].

From these considerations we understand that selection rules in the naïve diquark model should be found to limit the number of pentaquark states as well. On the other hand the experimental situation is too preliminary to allow these kind of reasonings and comparisons. For this reason, the following section, which is devoted to the presentation of a different approach to the understanding of $X Y Z$ phenomenology, does not contain any reference to pentaquarks.

What we can learn from the recent observation of pentaquark resonances is again the indication that diquarks must be good degrees of freedom to describe the nature of exotic hadronic resonances. A new study on the diquark structure of pentaquarks which revises some of the conclusions drawn here is found in [119].

\section{Tetraquarks and diquarks in the $1 / N$ expansion}

This Section is especially based on a work by Maiani et al. on tetraquarks in the $1 / N$ expansion [9], where $N$ is the number of colors, following a stream of papers on the same subject initiated by Weinberg [120]. The contributions to this discussion by Knecht and Peris [121] and Cohen and Lebed [122] where also particularly useful to us.

The reputation of tetraquarks was somehow obscured by a theorem by S. Coleman and E. Witten [123, 124] stating that: tetraquarks correlators for $N \rightarrow \infty$ reduce to disconnected meson-meson propagators. The theorem follows from the simple fact that a four quark operator can be reduced to products of color singlet bilinears. Connecting each bilinear with itself, one gets two disconnected one-loop diagrams, i.e. a result of order $N^{2}$, while connected tetraquark diagrams are one-loop, thus of order $N$.

The argument was reexamined in [120], where it is argued that if connected tetraquark correlators develop a pole, it will be irrelevant that its residue is subleading with respect to the disconnected parts. After all, meson-meson scattering amplitudes are of order $1 / N$, in the $N \rightarrow \infty$ limit, but we do not consider mesons to be free particles.

The real issue, according to Weinberg, is the width of the tetraquark pole: if it increases for large $N$, the state will be undetectable for $N \rightarrow \infty$. Weinberg finds that decay rates scale $\sim 1 / N$ in the large $N$ expansion, making tetraquarks a respectable possibility. We will show that widths are even narrower than this. The tetraquark-meson 'decoupling' we find here will be functional to the discussion in Section 6.

After a concise review of the $1 / N$ expansion in QCD we will show that tetraquark poles should appear at non-planar orders of the $1 / N$ expansion [9]. In particular we are interested in the role of diquarks as tetraquark constituents. Their special role is discussed in Section 5.3. The results obtained in Section 5.3 will be used extensively in Section 6.

For a comprehensive review on the papers which followed Weinberg's remark [120-122, 125-127] we also refer to [4].

\subsection{1/N expansion in QCD: a short reminder}

The behavior of QCD for $N \rightarrow \infty$ has been characterized by G. 't Hooft [128]. Consider the gluon self-energy diagram in Figure 10 with external gluon colors fixed to $\bar{a}, \bar{b}$.

This diagram involves the product

$$
\sum_{c, d} f^{\bar{a} c d} f^{c d \bar{b}}=\operatorname{Tr}\left(T_{\mathrm{adj}}^{\bar{a}} T_{\text {adj }}^{\bar{b}}\right)=N \delta^{\bar{a} \bar{b}}
$$




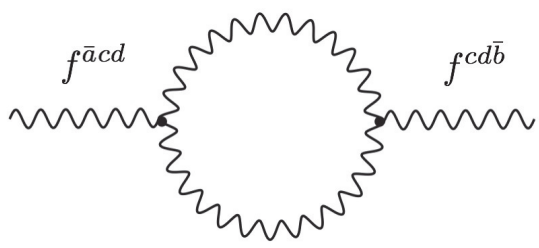

Figure 10: Gluon self-energy diagram with fixed colors $\bar{a}=\bar{b}$.

in the adjoint representation of $\mathrm{SU}(\mathrm{N})$. The gluon loop therefore contains a multiplicity factor of $N$ in $\mathrm{SU}(\mathrm{N})$. To make the large $N$ limit of this diagram finite, one requires that the couplings at vertices, $g_{\text {QCD }}$, scale with $N$ as $g_{\text {QCD }}=\sqrt{\lambda / N}$ so that

$$
\frac{\lambda}{N} \times N=\lambda \text { independent of } N
$$

where $\lambda$ is known as 't Hooft coupling. The large- $N$ limit is obtained keeping $\lambda$ fixed.

The gluon field is characterized by the color indices

$$
\left(A_{\mu}\right)_{j}^{i}=A_{\mu}^{a}\left(T^{a}\right)_{j}^{i}
$$

The number of independent components of this matrix in $\mathrm{SU}(\mathrm{N})$ are $N^{2}-1$. In the large $N$ limit however we can neglect the traceless condition, and treat it as a $N \times N$ matrix, with $N^{2}$ independent real components $A_{\mu}^{a}$, and represent the gluon line by a double color line — carrying a pair of color indices $i, j$. With this notation the diagram in Figure 10 can be represented as in Figure 11.

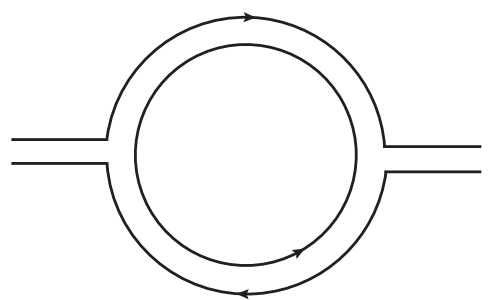

Figure 11: Gluon self-energy diagram in the large $N$. With this notation the multiplicity factor $N$ traced above in the $f$ structure constants, has a clear origin in the color loop at the center.

The origin of the multiplicity factor discussed above becomes apparent in the double-line notation: the factor of $N$ arises from the color loop in Fig. 11 and the factors of $1 / \sqrt{N}$ at the vertices make the large $N$ limit smooth. From the same figure we argue that the quark-gluon coupling will therefore also scale as $1 / \sqrt{N}$ and the quadrilinear gluon coupling as $1 / N$.

't Hooft shows that in the $N \rightarrow \infty$ limit, the quark lines have to be at the edge of the diagram (valence quarks), whereas diagrams with internal quark lines are suppressed (quenched theory). Internal gluons are instead allowed, but only planar diagrams, where gluon lines do not step over each other, survive at leading order.

Let us consider the correlation function of a color singlet quark bilinear. With no gluon lines, the result is obviously proportional to $N$, the number of colors running in the loop. A gluon line traversing the loop, see Figure 12, can be represented by two color lines running in opposite directions and joining the quark and antiquark lines that flow in the vertex. Thus we get two loops, i.e. a factor of $N^{2}$, times the factor $1 / N$, therefore a contribution of order $N$ again. The 


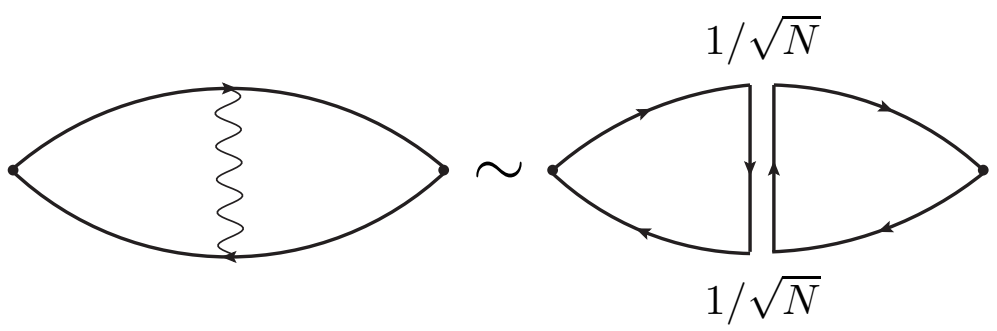

Figure 12: One gluon exchange correction to the correlation function of a color singlet quark bilinear, represented by the open circle. Representing the gluon line by two, oppositely running lines joining the quark lines on the edge, one sees that the diagram reduces, for color number counting, to a two loop diagram. Thus one recovers a result of order $N$, like the lowest order diagram, multiplied by the color reduced coupling $\lambda$.

same adding trilinear or quadrilinear gluon vertices. As long as gluons are inserted within the the surface delimited by the external quark lines and as long as they are planar, the $N$ power counting does not change. Non-planar gluons, as we will see, are instead subleading.

The sum of all planar diagrams of this kind will again be of order $N$, times a non-perturbative function of $\lambda$, which may well develop poles for certain values of the external momentum, $q^{2}$.

The sum of all planar diagrams like the one on the $l h s$ of Figure 12 is represented by

$$
\left\langle 0\left|J(p) J^{\dagger}(p)\right| 0\right\rangle \sim N
$$

where the operator $J^{\dagger}$ acts on the vacuum to create a meson state, and

$$
\left\langle 0\left|J(p) J^{\dagger}(p)\right| 0\right\rangle=\sum_{n} \frac{\langle 0|J(p)| n\rangle\left\langle n\left|J^{\dagger}(p)\right| 0\right\rangle}{p^{2}-m_{n}^{2}}=\sum_{n} \frac{f_{n}^{2}}{p^{2}-m_{n}^{2}}
$$

with the decay constant $f_{n}=\langle 0|J(p)| n\rangle$.

The behavior at large momenta of $\left\langle 0\left|J(p) J^{\dagger}(p)\right| 0\right\rangle$ is expected to be logarithmic ${ }^{27}$. However the sum over meson states in Eq. (5.5) can scale as $\sim \ln p^{2}$, at large $p^{2}$, only if it has an infinite number of terms, as can be seen by substituting $\sum_{n} \rightarrow \int d m_{n}^{2}$. Thus we have an infinite number of poles, corresponding to a tower of (stable) meson states in the correlation function $\left\langle 0\left|J(p) J^{\dagger}(p)\right| 0\right\rangle$. These have a given flavor content, e.g. quarkonium mesons with varying spin and parity. Meson masses are independent of $N$ and the entire $N$ dependency of the rhs of (5.5) is encoded in $f_{n}$. In the case at hand this means that each $f_{n} \sim \sqrt{N}$.

In the strict $N \rightarrow \infty$ limit, mesons are non-interacting particles and hence they are stable. This can be seen by considering a three-point function, a loop like the one in Fig. 12 with three meson insertions. The color loop of order $N$ has to be confronted with $f_{n}^{3} g$, so that $g \sim 1 / \sqrt{N}$.

\subsection{Tetraquarks in the $1 / N$ expansion}

\subsubsection{From planar to non-planar diagrams}

Following [121], diagrams with tetraquark operator insertions can be depicted as in Figure 13, where a two-point correlation function of a neutral tetraquark is represented. We do not include diagrams with heavy quark annihilations, which are expected to be suppressed (OZI rule).

\footnotetext{
${ }^{27}$ The asymptotic behavior of the correlators is indeed driven by asymptotic freedom, and apart of a factor $p^{n}$ given by dimensional analysis, it is expected to scale with fractional powers of logarithms, according to the anomalous dimension of $J$, see [129].
} 


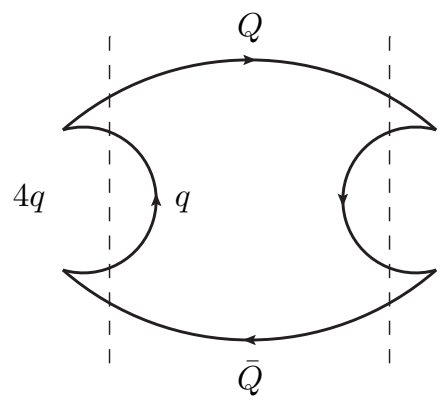

Figure 13: Free two-point correlation function of a tetraquark meson.

The dashed lines represent the cuts where tetraquarks could be found. Apparently tetraquark intermediate states are possible, along with tetraquark-meson mixings. This is basically the point of view developed in [121], following Weinberg in [120].

The simplest way to include color interactions in the tetraquark cut of Fig. 13 is that of adding planar gluons. Consider for example one planar gluon on the light quark line as in Fig. $14(A)$. Using the double line notation, Fig. $14(B)$, one sees that the tetraquark cut contains color disconnected components. This motivated the considerations in [9].
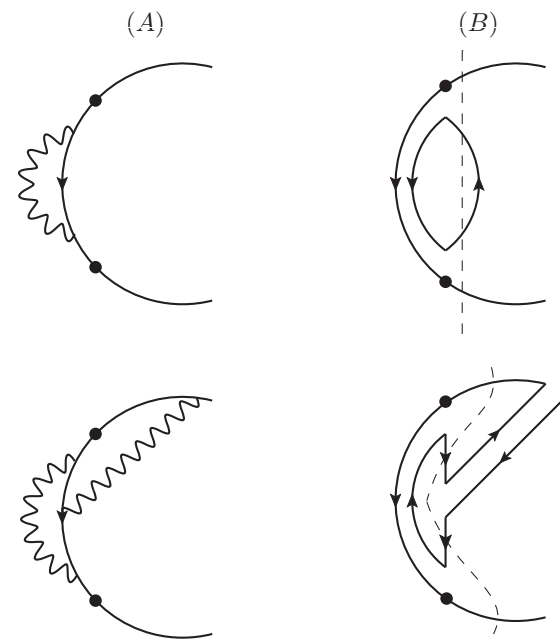

$(C)$

$(D)$

Figure 14: $(A)$ A piece of a correlation function with two quark bilinear insertions and a planar gluon. Except for the interaction gluon, like the left hand half of the diagram in Fig. 13 with the light quark line deformed to run on the perimeter of the diagram. Let the two bilinear insertions be $Q^{i} q^{k}$ and $\bar{Q}^{\ell} \bar{q}^{m}(i \neq k, \ell \neq m)$. (B) Double-line reperesentation of $(A)$. Cuts might only intercept color-disconnected four-quark configurations: the cut in figure is for example $Q^{i} q^{k} \bar{q}^{k} \bar{Q}^{r}$. (C) Non-planar diagram with a gluon running on the perimeter of the amplitude. (D) Double-line representation of $(C)$. Cuts might intercept connected four quark configurations like $Q^{i} q^{3} \bar{Q}^{r} \bar{q}^{3}$ in figure. Although $(D)$ can topologically be deformed into a simple loop diagram — which is what one usually do to count external color loops. Closing the right end half of the loop, the order in the $1 / N$ expansion is $N \times(1 / \sqrt{N})^{4} \sim 1 / N$ instead of $N$. Notice alternatively that diagram $(A)$ is of order $T^{a} T^{a} \times(1 / \sqrt{N})^{2} \sim N$ whereas $(C)$ is $T^{a} T^{b} T^{a} T^{b} \times(1 / \sqrt{N})^{4} \sim 1 / N \times N^{2} \times 1 / N^{2} \sim 1 / N$ i.e. $1 / N^{2}$ with respect to $(A)$.

The main content of [9] is that non-planar diagrams may provide cuts with color correlated tetraquarks. In essence this can be seen in Fig. $14(C)$, whose double line notation version, $(D)$, shows that this is a possibility. Diagrammatic considerations of this kind do not provide a proof that tetraquark cuts are indeed found in the non-planar approximation, but at least suggest that tetraquarks are more strongly decoupled from mesons than expected. 


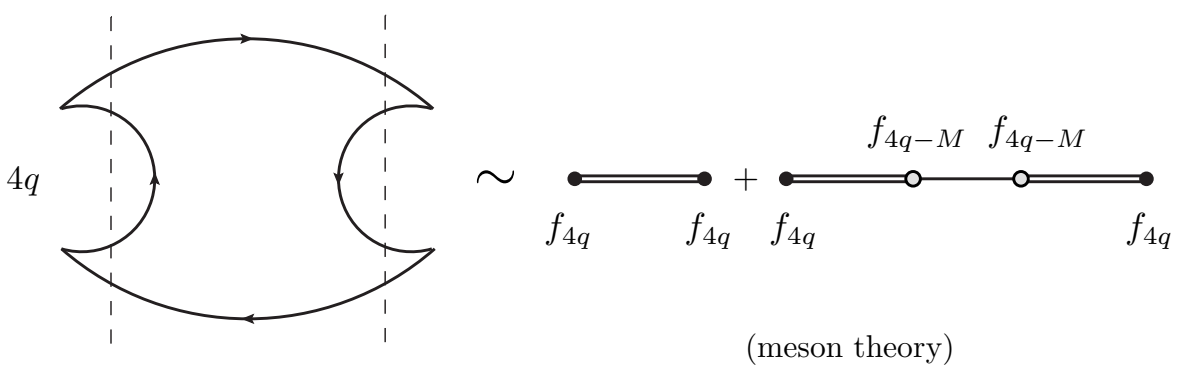

Figure 15: The same diagram as in Figure 13 with its counterpart in meson theory on the $r h s$. The diagram on the $l h s$ is of order $N$ therefore $f_{4 q}$ in the meson theory is of order $\sqrt{N}$ and the mixing $f_{4 q-M} \sim N^{0}$. This counting relies on the fact that we might have only the tetraquark propagator in the meson theory. If we assume instead that the loop diagram only involves $4 q-M$ mixings we would simply get $f_{4 q}^{2} f_{4 q-M}^{2} \sim N$. In other words, diagrams on the rhs are meant to be summed on meson/tetraquarks intermediate states. This eventually generates a contact term in the second diagram on the $r h s$, which is represented by the first diagram. This means that $f_{4 q}$ in the first diagram is equivalent to $f_{4 q} f_{4 q-M}$ in the second. In the following we will consider mostly diagrams of the type of the second one on the rhs.

The non planar gluon in Fig. 14, is like a handle applied to the diagram and the amplitude of Figure 14 goes like $1 / N$, as can be checked by direct calculation.

Even though we cannot provide a proof that diagrams with one handle have indeed a tetraquark pole, we find that different correlation functions are consistent with the factorization of residues at the pole, as we discuss below.

Let us consider again the diagram in Figure 13, and the corresponding effective meson theory diagrams, as done in Figure 15. Since the quark loop diagram is of order $N$ (whatever planar gluon interactions might be inserted), the

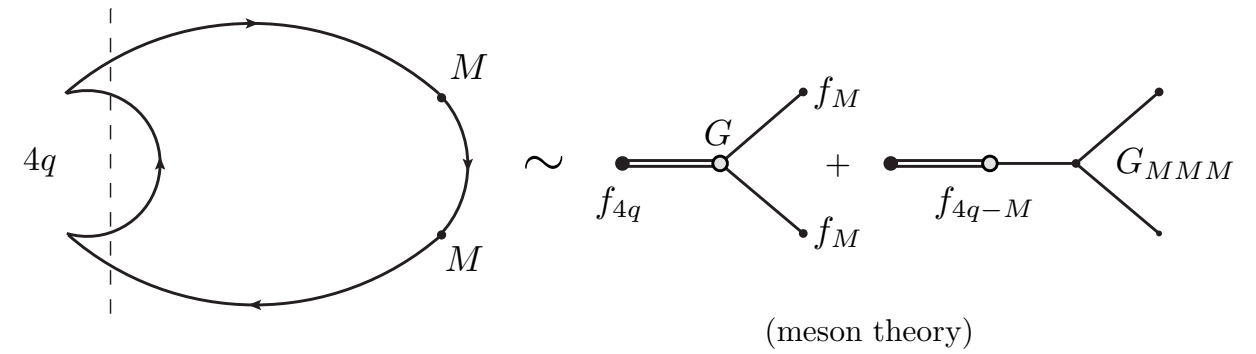

Figure 16: The coupling $G$ is found to be of order $G \sim 1 / \sqrt{N}$ to match the quark loop diagram, which is $\sim N$. Also $G \sim N^{0} \times 1 / \sqrt{N}$ using the mixing in Figure 15. Because of this either writing the diagram with no $4 q-M$ mixing and $G$ or writhing the diagram with mixing and $f_{4 q-M} / \sqrt{N}$ is the same thing. The $N$ order of the diagram will be $f_{4 q} f_{4 q-M} / \sqrt{N}(\sqrt{N})^{2} \sim N$ or $f_{4 q} f_{4 q-M} / \sqrt{N} \sim 1$. Also here, as commented in Figure 15 , the first diagram on the rhs corresponds to the contact term generated by the infinite sum over mesons on the second diagram. This in turn corresponds to the identification $G=f_{4 q-M} 1 / \sqrt{N}$.

$f_{4 q}$ decay constant $f_{4 q}=\langle 0|J| 4 q\rangle$ has to be such that $f_{4 q}^{2} \sim N$ or (see discussion around Eq. (5.5))

$$
f_{4 q} \sim \sqrt{N}
$$

Since the $r h s$ in Figure 15 also scales as $f_{4 q}^{2} f_{4 q-M}^{2}$, the latter requires the tetraquark-meson mixing to be

$$
f_{4 q-M} \sim N^{0}
$$

We might use the result found for $f_{4 q}$ in the three-point function responsible for the decay of a tetraquark into two mesons. The $4 q-M M$ vertex contains the coupling $G$ and $G f_{4 q} f_{M}^{2} \sim N$ to match the $N$ order in the loop diagram see Figure 16. Since $f_{M} \sim \sqrt{N}$, we have that $G \sim 1 / \sqrt{N}$; this can also be seen through the $4 q-M$ mixing of the $r h s$ 
of Figure 16. It goes like $G \sim f_{4 q-M} G_{M M M} \sim N^{0} \times 1 / \sqrt{N}$, from the fact that the trilinear meson vertex scales as $G_{M M M} \sim 1 / \sqrt{N}$.

The conclusion that

$$
G \sim 1 / \sqrt{N}
$$

reached in [120], implies that tetraquarks will be narrow resonances in the large $N$ limit. In other words they are states which in principle might be observed as narrow resonances, contrarily to the fall apart decay which is intrinsic in the Coleman-Witten argument mentioned above. In these respects if connected tetraquark correlators develop a pole, it will be irrelevant that its residue is of the subleading order $N$ (instead of $N^{2}$ ). What is really important is that the total width will scale as $\Gamma \sim 1 / N$.

If we assumed that only $4 q-M$ mixings are allowed in meson theory, and that the first term on the $r h s$ of Figure 15 is the contact term resulting from a (infinite) sum over the mesons on the second term, we would simply have $f_{4 q}^{2} f_{4 q-M}^{2} \sim$ $N$ which means

$$
f_{4 q} \sim \frac{\sqrt{N}}{f_{4 q-M}}
$$

leading to

$$
G \sim \frac{f_{4 q-M}}{\sqrt{N}}
$$

consistent with the second term on the rhs of Figure 16. To compute the order of $G$ in the large $N$ expansion, the mixing $f_{4 q-M}$ should be computed from another diagram. Consider the two-point $M M$ correlator in Figure 17 where

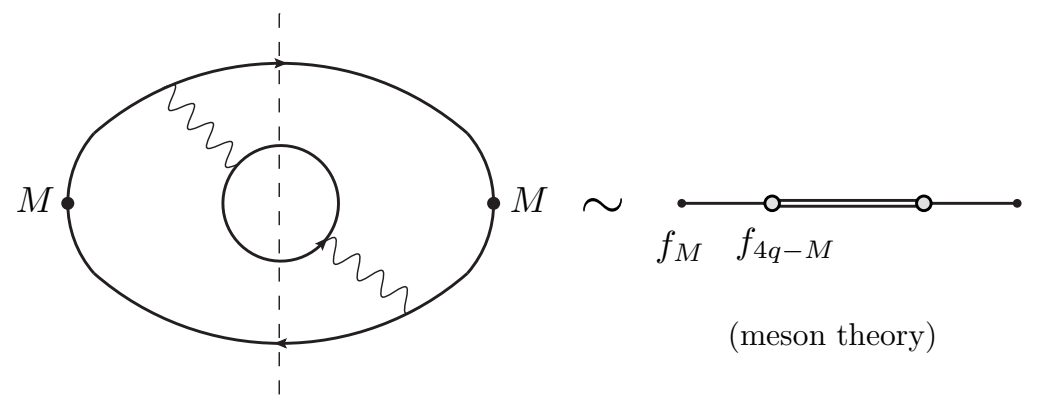

Figure 17: The diagram on the $l h s$ is of order $N^{0}$ therefore $f_{4 q-M} \sim 1 / \sqrt{N}$, which is not consistent with $f_{4 q-M} \sim N^{0}$ in Figure 15 .

it is found that

$$
f_{4 q-M} \sim 1 / \sqrt{N}
$$

where it is used the standard result $f_{M} \sim \sqrt{N}$. This leads to $G \sim 1 / N$.

From what just found, we observe an inconsistency. In fact, from Figure 15 one deduces $f_{4 q-M}^{2} \sim N^{0}$, while from Figure 17 is found that $f_{4 q-M}^{2} \sim 1 / N$.

This inconsistency is removed if tetraquark decay and mixing constants are extracted from non-planar diagrams as shown in the next Section.

\subsubsection{Non-planar diagrams}

We suggest that tetraquark poles, if present, should appear in non-planar diagrams. The correct way to extract the decay constant involving a four-quark state is therefore to repeat the previous analysis to the first non-planar order. We 
consider the following diagram in Figure 18, which contains one handle. The $1 / N$ order is determined by

$$
N^{2-L-2 H}
$$

where $L$ is the number of quark loops and $H$ is the number of handles. In the case in Figure 18 we have $L=2, H=1$ giving $N^{-2}$, as can be seen also using the standard perturbative counting rules introduced in Figure 12.

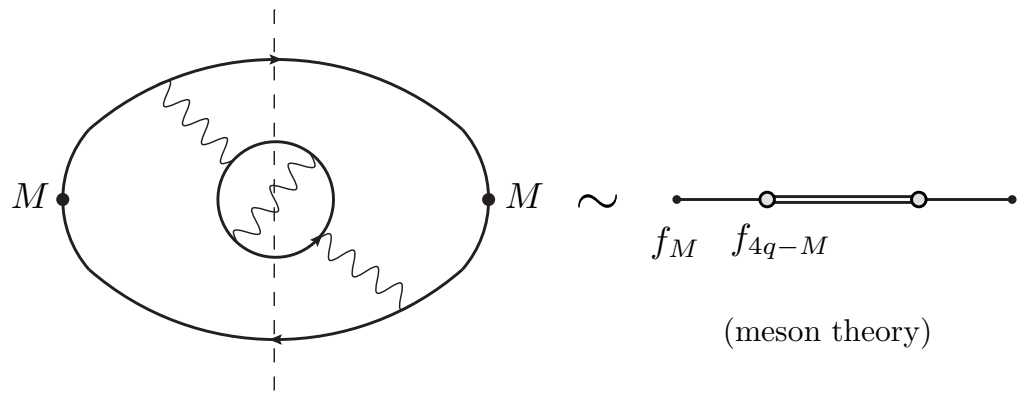

Figure 18: In this non-planar diagram the quark loop order is $1 / N^{2}$ and $f_{M}^{2} f_{4 q-M}^{2} \sim 1 / N^{2}$, giving $f_{4 q-M} \sim 1 /(N \sqrt{N})$ instead of $N^{0}$ as in Figure 17 .

From the counting of powers in Figure 18 it results that

$$
f_{4 q-M} \sim 1 /(N \sqrt{N})
$$

We can determine the $f_{4 q}$ decay constant from the non-planar two-point correlator of the tetraquark. In the meson theory we have the $4 q-M$ mixings - contact terms are inessntial to our discussion. The non-planar diagram in Figure 19 is of order $1 / N^{3}=N^{2-1-2 \times 2}$, which corresponds to $f_{4 q-M}^{2}$. Therefore we need

$$
f_{4 q} \sim N^{0}
$$

We must check if the power counting leading to $f_{4 q} \sim N^{0}$ does not produce conflicting results in other correlation

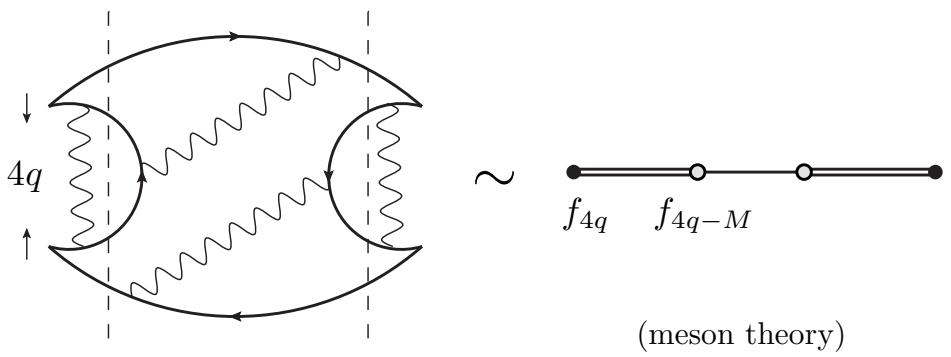

Figure 19: This diagram is of order $1 / N^{3}$ thus, in the meson theory we have $f_{4 q}^{2} f_{4 q-M}^{2} \sim 1 / N^{3}$. Using $f_{4 q-M}=1 /(N \sqrt{N})$ from Figure 18 , we get $f_{4 q} \sim N^{0}$ (in place of $f_{4 q} \sim \sqrt{N}$ found in Figure 15).

functions.

For this purpose consider the trilinear correlator giving rise to the tetraquark decay into two mesons. This is represented in Figure 20 to the non planar order with one handle. We find that

$$
G \sim 1 / N^{2}
$$




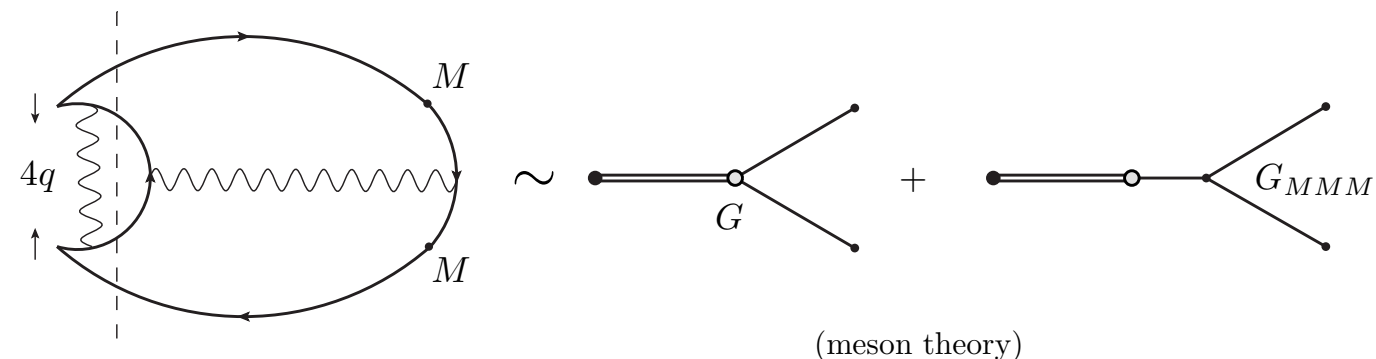

Figure 20: The quark loop diagram is of order $1 / N$. Therefore $f_{4 q} G(\sqrt{N})^{2}=f_{4 q} f_{4 q-M} 1 / \sqrt{N}(\sqrt{N})^{2} \sim 1 / N$ gives $G \sim 1 / N^{2}$, taking $f_{4 q} \sim N^{0}$. Also here, as commented in Figure 15, the first diagram on the rhs corresponds to the contact term generated by the infinite sum over mesons on the second diagram. This in turn corresponds to the identification $G=f_{4 q-M} 1 / \sqrt{N}$.
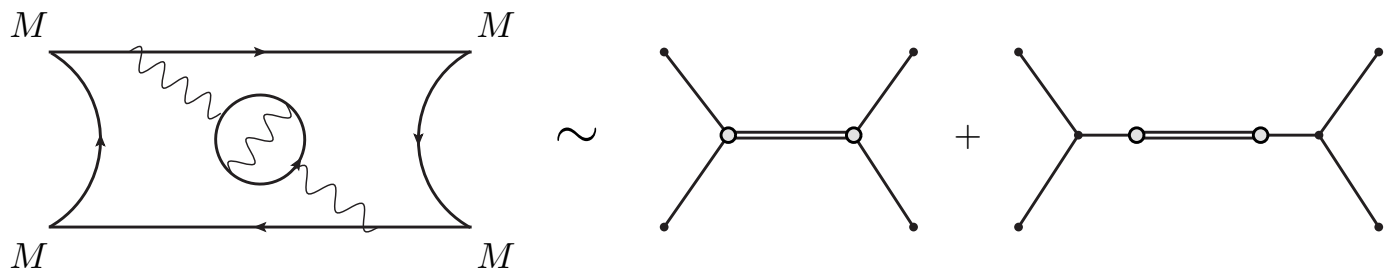

(meson theory)

Figure 21: A consistency check. Here we can use that $G \sim 1 / N^{2}$ and $f_{4 q-M}=f_{M-4 q}=1 /(N \sqrt{N})$ to verify that $G^{2}(\sqrt{N})^{4}=$ $f_{M-4 q}^{2}(1 / \sqrt{N})^{2}(\sqrt{N})^{4}=1 / N^{2}$ as in the quark-loop diagram — we used $G \sim 1 / N^{2}$ as found in Figure 20 . Also here, as commented in Figure 15, the first diagram on the rhs corresponds to the contact term generated by the infinite sum over mesons on the second diagram. This in turn corresponds to the identification $G=f_{4 q-M} 1 / \sqrt{N}$.

using $f_{4 q}$ and $f_{4 q-M}$ introduced above. As a consistency check we also analyze the $M M \rightarrow M M$ four-point function finding a match between the $N$ order in the loop diagram and in the meson theory, the decay and mixing constants being fixed. This is done in Figure 21.

Therefore we conclude that the tetraquark states can be even narrower than what expected at the order $N$, since we found $G \sim 1 / N^{2}$ in place of $G \sim 1 / \sqrt{N}$. With this approach we consistently find $f_{4 q-M}^{2} \sim 1 / N^{3}$.

\subsubsection{Charged tetraquarks}

The determination of the coupling $G$ is different if charged tetraquarks are taken into account. Consider for example the two-point function containing the charged tetraquark $c u \bar{c} \bar{d}$, in Figure 22 - i.e. assume that the amplitude in Fig. 22 connects a hidden-charm+light meson $c \bar{c}+u \bar{d}$ pair to two open-charm mesons $c \bar{d}+u \bar{c}$. Like in the cases examined previously, the four quarks in the cut are found in color disconnected configurations at the planar order, whereas color connections are forced in non-planar diagrams, see Fig. 23.

The same topology can be used to represent a two-point function of tetraquark operators, $4 q \rightarrow 4 q$, or a $4 q \rightarrow M M$ correlator. In the first case it is obtained that $f_{4 q} \sim 1 / \sqrt{N}$, since the graph with one handle is of order $1 / \sqrt{N}$. In the second case

$$
f_{4 q} G f_{M}^{2} \sim 1 / N \Rightarrow G \sim 1 /(N \sqrt{N})
$$

Neutral tetraquarks found in the cuts of Fig. 19 can mix with meson-meson states, see the rhs of Fig. 19. Such mixings are not present when we come to topologies as the one in Fig. 23. Therefore charged tetraquarks, which do not mix with mesons, tend to be broader than neutral ones, which do. Indeed we see that $G_{\text {charged }} \sim 1 /(N \sqrt{N})=1 / f_{4 q-M} G_{\text {neutral }}$. 


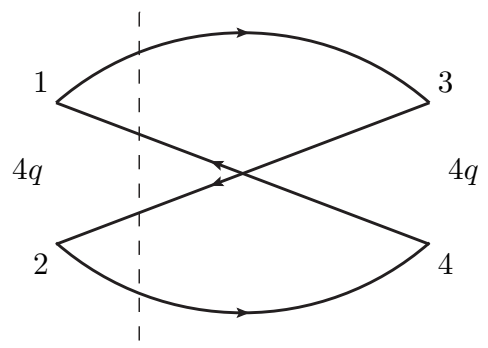

Figure 22: Charged tetraquark two-point correlation function. This might be untwisted in a $M M \rightarrow M M$ meson-meson amplitude, with no tetraquarks in the $s$-channel.

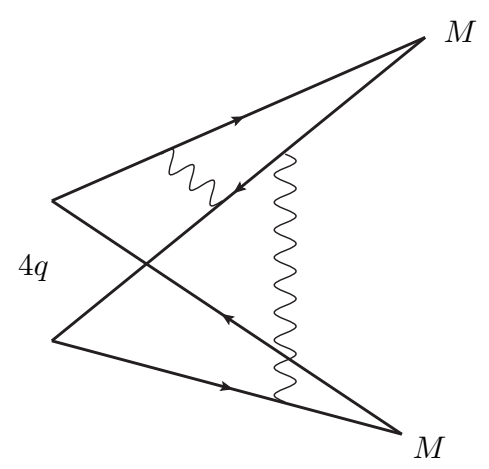

Figure 23: Decay of a charged tetraquark in two mesons in the lowest non-planar diagram. Following the same reasonings from this topology we see that i) $f_{4 q}^{2} \sim 1 / N$ and ii) $f_{4 q} G(\sqrt{N})^{2} \sim 1 / N$ thus $G \sim 1 /(N \sqrt{N}$ ) (see text for more details) differently from what found for neutral tetraquarks $\left(G \sim 1 / N^{2}\right.$ in Figure 20).

Without the color connections present at non-planar orders, these amplitudes would be saturated by meson-meson channels, as observed at the beginning of this Section. Diagrammatic arguments are not a proof of the existence of tetraquark poles, but an indication of the fact that they might occur at non-planar orders. However what found indicates that tetraquarks are reluctant to mix with meson states.

As we will see in the next subsection, any diquarkonium tetraquark can be written in terms of its meson-meson quantum numbers (Fierz rearrangement) but this does not contain any dynamical information about the probability amplitude of a compact tetraquark to rearrange its quark content and decay into a meson-meson state (or viceversa) or to mix with a meson state. $1 / N$ expansion is used to shed some light on these aspects.

Taking the large $N$ considerations at their extreme consequences $(N \rightarrow \infty)$, the fact that the $4 q-M$ and $4 q-M M$ mixings are found to be rather suppressed at large $N$ would make the observed prominent decay modes very unlikely. However $N=3$ and the numerators of the $1 / N$ expansion are unknown.

More interestingly for us, an enhancement mechanism of the mixing, as that proposed in Section 6, would explain why some of these very narrow states are being observed in experiments.

\subsection{Diquarkonia in the $1 / N$ expansion}

For $N$ strictly infinite, $q \bar{q}$ mesons generated by quark bilinear correlators are free particles. Interactions are generated by letting $N$ to be large but finite. Irreducible vertices with $k$ external mesons are of order $N^{1-k / 2}$. 
We refer to Figure 22 for the flavor composition of a charged tetraquark. Non-planar quark diagrams with the same flavor structure, as in Figure 23, would develop tetraquark poles in the $s$-channel contributing an amplitude of order $G \sim 1 /(N \sqrt{N})$.

We consider $S$-wave meson-meson scattering in the channel with $J^{P}=1^{+}$and the the three pairs of 'in' pseudoscalar and vector mesons $\left(M_{1} M_{2}\right)$ together with the three pairs of 'out' open charm mesons $\left(M_{3} M_{4}\right)$

$$
\begin{aligned}
& M_{1} M_{2}=\eta_{c} \rho^{+}, \psi \pi^{+}, \psi \rho^{+} \\
& M_{3} M_{4}=\bar{D}^{0} D^{*+}, D^{+} \bar{D}^{* 0}, \bar{D}^{* 0} D^{*+}
\end{aligned}
$$

As commented above, a diagram like the one in Figure 22 might be interpreted as a meson-meson $M M \rightarrow M M$ scattering diagram converting hidden charm+light mesons into open charm ones.

To appropriately consider the quantum numbers of the $M_{1,2,3,4}$ mesons, we have to insert the spin matrices of each of them (written as $\bar{q} \Gamma_{i} q^{\prime}$ ) in the vertices, and obtain the spin factor ${ }^{28}$

$$
S(12 ; 34)=\operatorname{Tr}\left(\Gamma_{1} \Gamma_{3}^{\dagger} \Gamma_{2} \Gamma_{4}^{\dagger}\right) \equiv\left\langle\Gamma_{1} \Gamma_{3}^{\dagger} \Gamma_{2} \Gamma_{4}^{\dagger}\right\rangle
$$

The order in the trace follows backward the arrows in the diagram of Figure 22.

The quark diagram is such that it transforms the hidden charm, into open charm channels. If we take for example $M_{1} M_{2}=\eta_{c} \rho^{+}$and $M_{3} M_{4}=\bar{D}^{0} D^{*+}$, we obtain

$$
S^{a b}\left(\eta_{c} \rho^{+} ; \bar{D}^{0} D^{*+}\right)=C\left\langle\sigma^{2} \cdot \sigma^{2} \cdot \sigma^{2} \sigma^{a} \cdot\left(\sigma^{2} \sigma^{b}\right)^{\dagger}\right\rangle=C\left\langle\sigma^{a} \sigma^{b}\right\rangle=2 C \delta^{a b}
$$

and the same for $\eta_{c} \rho^{+} \leftrightarrow D^{+} \bar{D}^{* 0}$

$$
S^{a b}\left(\eta_{c} \rho^{+} ; D^{+} \bar{D}^{* 0}\right)=2 C \delta^{a b}
$$

For $\eta_{c} \rho^{+} \leftrightarrow \bar{D}^{* 0} D^{*+}$, we obtain

$$
S^{a b}\left(\eta_{c} \rho^{+} ; \bar{D}^{* 0} D^{*+}\right)=C^{\prime}\left\langle\sigma^{2} \cdot \sigma^{d} \sigma^{2} \cdot \sigma^{2} \sigma^{a} \cdot \sigma^{c} \sigma^{2}\right\rangle\left(-i \epsilon^{b c d}\right)=4 C^{\prime} \delta^{a b}
$$

According to the definitions in Eqs. (4.35) $C=1 / 2$ and $C^{\prime}=1 / \sqrt{2}$. We can then complete the spin factor matrix of hidden $(H)$ to open charm $(O)$ transitions $H \leftrightarrow O$

$$
\begin{array}{llcc} 
& \bar{D}^{0}\left(D^{*+}\right)^{b} & D^{+}\left(\bar{D}^{* 0}\right)^{b} & \left(\bar{D}^{* 0} \times D^{*+}\right)^{b} \\
S^{a b}(H ; O)= & \eta_{c}\left(\rho^{+}\right)^{a} & \psi^{a} \pi^{+} \\
& \left(\psi \times \rho^{+}\right)^{a}
\end{array}\left(\begin{array}{ccc}
1 & \sqrt{2} \\
1 & 1 & -\sqrt{2} \\
\sqrt{2} & -\sqrt{2} & 0
\end{array}\right) \cdot \frac{1}{2} \delta^{a b}
$$

The first row is explicitly given by (5.20), (5.21), (5.22).

The state in the cut must be a superposition of the 'in' state and the 'out' one to project on both. If we fix the quantum numbers of the 'in' state to be $\left(I^{G}\right) J^{P C}=\left(1^{-}\right) 1^{++}$, we are choosing $M_{1} M_{2}=\psi \times \rho^{+}$. The out state can then be the superposition $\bar{D}^{0} D^{*+}-D^{+} \bar{D}^{* 0}$ (last row of the above matrix), which happens to have the right $J^{P C}$ quantum numbers. For the sake of brevity, from now one we will omit the normalization factors and assume that the hadronic states are everywhere normalized to one. We then find that the state in the cut of Figure 22 should be

$$
\psi \times \rho^{+} \pm\left(\bar{D}^{0} D^{*+}-D^{+} \bar{D}^{* 0}\right)
$$

\footnotetext{
${ }^{28} \gamma$-matrices associated to the quark gluon interaction $i$ ) do not count since they reduce to $\gamma^{0}= \pm 1$, in the non-relativistic limit.
} 
But this, apart from phases, corresponds to the $X^{+}$meson written as a compact diquarkonium (to make sure that attraction in diquarks makes sense in the $1 / N$ expansion we refer to Appendix B)

$$
\begin{aligned}
X^{+} & =[c u]_{S=0}[\bar{c} \bar{d}]_{S=1}+[c u]_{S=1}[\bar{c} \bar{d}]_{S=0}= \\
& =\left(\epsilon_{\gamma \alpha \beta} c^{\alpha} \sigma^{2} u^{\beta}\right)\left(\epsilon^{\gamma \alpha^{\prime} \beta^{\prime}} \bar{c}_{\alpha^{\prime}} \sigma^{2} \boldsymbol{\sigma} \bar{d}_{\beta^{\prime}}\right)+\left(\sigma^{2} \leftrightarrow \sigma^{2} \sigma\right)= \\
& =\left(c^{\alpha} \sigma^{2} u^{\beta}\right)\left[\left(\bar{c}_{\alpha} \sigma^{2} \boldsymbol{\sigma} \bar{d}_{\beta}\right)-\left(\bar{c}_{\beta} \sigma^{2} \sigma \bar{d}_{\alpha}\right)\right]+\left(\sigma^{2} \leftrightarrow \sigma^{2} \sigma\right)= \\
& =-i\left(c \sigma^{2} \boldsymbol{\sigma} \bar{c}\right) \times\left(u \sigma^{2} \boldsymbol{\sigma} \bar{d}\right)-\left[\left(c \sigma^{2} \bar{d}\right)\left(u \sigma^{2} \boldsymbol{\sigma} \bar{c}\right)-\left(c \sigma^{2} \boldsymbol{\sigma} \bar{d}\right)\left(u \sigma^{2} \bar{c}\right)\right]= \\
& \sim-i \psi \times \rho^{+}+\left(D^{+} \bar{D}^{* 0}-\bar{D}^{0} D^{*+}\right)
\end{aligned}
$$

In the first row we have used the definition of the $X^{+}\left(J^{P C}=1^{++}\right)$state in terms of diquarks, in the second we have assumed attraction in the antisymmetric color representation (see Appendix B), in the fourth we neutralize color composing color singlets and used the Fierz rearrangement results from the Table 6 and $\left(\sigma^{2} \sigma\right)^{T}=\sigma^{2} \sigma,\left(\sigma^{2}\right)^{T}=-\sigma^{2}$. In addition to the results in Table 6 , other useful relations which will be needed in the following, are listed below

\begin{tabular}{c|ccc}
\hline \hline- & $\left(\sigma^{2}\right)_{i k}\left(\sigma^{2} \boldsymbol{\sigma}\right)_{j l}$ & $\left(\sigma^{2} \boldsymbol{\sigma}\right)_{i k}\left(\sigma^{2}\right)_{j l}$ & $i\left(\sigma^{2} \boldsymbol{\sigma}\right)_{i k} \times\left(\sigma^{2} \boldsymbol{\sigma}\right)_{j l}$ \\
\hline$\left(\sigma^{2}\right)_{i j}\left(\sigma^{2} \boldsymbol{\sigma}\right)_{k l}$ & $+1 / 2$ & $-1 / 2$ & $+1 / 2$ \\
$\left(\sigma^{2} \boldsymbol{\sigma}\right)_{i j}\left(\sigma^{2}\right)_{k l}$ & $-1 / 2$ & $+1 / 2$ & $+1 / 2$ \\
$i\left(\sigma^{2} \boldsymbol{\sigma}\right)_{i j} \times\left(\sigma^{2} \boldsymbol{\sigma}\right)_{k l}$ & +1 & +1 & 0 \\
\hline \hline
\end{tabular}

Table 6: Coefficients of the Fierz rearrangement of $J^{P}=1^{+}$quadrilinears. Notice that the square of this matrix is equal to the identity.

$$
\left(\sigma^{2} \boldsymbol{\sigma}\right)_{i j} \cdot\left(\sigma^{2} \boldsymbol{\sigma}\right)_{k l}=\frac{3}{2}\left(\sigma^{2}\right)_{i l}\left(\sigma^{2}\right)_{k j}-\frac{1}{2}\left(\sigma^{2} \sigma\right)_{i l} \cdot\left(\sigma^{2} \boldsymbol{\sigma}\right)_{k j}
$$

or

$$
\left(\sigma^{2} \sigma\right)_{i j} \cdot\left(\sigma^{2} \sigma\right)_{k l}=-\frac{3}{2}\left(\sigma^{2}\right)_{i k}\left(\sigma^{2}\right)_{j l}-\frac{1}{2}\left(\sigma^{2} \sigma\right)_{i k} \cdot\left(\sigma^{2} \sigma\right)_{j l}
$$

together with

or

$$
\left(\sigma^{2}\right)_{i j}\left(\sigma^{2}\right)_{k l}=\frac{1}{2}\left(\sigma^{2}\right)_{i l}\left(\sigma^{2}\right)_{k j}+\frac{1}{2}\left(\sigma^{2} \sigma\right)_{i l} \cdot\left(\sigma^{2} \sigma\right)_{k j}
$$

$$
\left(\sigma^{2}\right)_{i j}\left(\sigma^{2}\right)_{k l}=\frac{1}{2}\left(\sigma^{2}\right)_{i k}\left(\sigma^{2}\right)_{j l}-\frac{1}{2}\left(\sigma^{2} \sigma\right)_{i k} \cdot\left(\sigma^{2} \sigma\right)_{j l}
$$

Similarly one can show that (see (4.56c))

$$
\left(\sigma^{2} \sigma^{(a}\right)_{i j}\left(\sigma^{2} \sigma^{b)}\right)_{k l}-\frac{2}{3}\left(\sigma^{2} \sigma^{c}\right)_{i j}\left(\sigma^{2} \sigma^{c}\right)_{k l}=\left(\sigma^{2} \sigma^{(a}\right)_{i k}\left(\sigma^{2} \sigma^{b)}\right)_{j l}-\frac{2}{3}\left(\sigma^{2} \sigma^{c}\right)_{i k}\left(\sigma^{2} \sigma^{c}\right)_{j l}
$$

A factor of two must be taken into account from each spinor exchange - notice the + sign of the spin exchanged term. Each time spinors are exchanged a factor of -1 is included: a term like $\left(u \sigma^{2} \bar{c}\right)=\left(\bar{c} \sigma^{2} u\right) \sim \bar{D}^{0}$. In the fifth row we assume a simplified hadronization of the quark bilinears (light spin might be not conserved), and, up to an overall normalization, restore the $1 / \sqrt{2}$ normalizations. We thus found that the 'eigenchannel' of matrix $S(H ; O)$ contains $X^{+}$ written as a diquarkonium state.

Let us consider the G-parity transformation properties of the second term on the fourth row of in (5.25). Recall that $G u=-\bar{d}, G d=\bar{u}$ and $G \bar{d}=u, G \bar{u}=-d$. Then we get that under G-parity

$$
\left[\left(c \sigma^{2} \bar{d}\right)\left(u \sigma^{2} \sigma \bar{c}\right)-\left(c \sigma^{2} \sigma \bar{d}\right)\left(u \sigma^{2} \bar{c}\right)\right] \rightarrow\left[\left(\bar{c} \sigma^{2}(-u)\right)\left(\bar{d} \sigma^{2} \sigma c\right)-\left(\bar{c} \sigma^{2} \sigma(-u)\right)\left(\bar{d} \sigma^{2} c\right)\right]
$$


but the latter term is equivalent to $(-1)$ times the term on the $l h s$ (because $\left(\sigma^{2}\right)^{T}=-\sigma^{2}$ and $\left(\sigma^{2} \sigma\right)^{T}=\sigma^{2} \sigma$ and a -1 is to be included each time spinors are exchanged). Therefore the $G$ parity is $G=-$, which means that the neutral state will have $C=+$. The same can be concluded just starting from $\left([c u]_{0}[\bar{c} \bar{d}]_{1}+[c u]_{1}[\bar{c} \bar{d}]_{0}\right)$. The $G$-parity analysis proceeds in the same way also for the two following terms.

Similarly we can write (notice the - sign in the spin exchanged term)

$$
\begin{aligned}
Z^{+} & =\left(c^{\alpha} \sigma^{2} u^{\beta}\right)\left[\left(\bar{c}_{\alpha} \sigma^{2} \boldsymbol{\sigma} \bar{d}_{\beta}\right)-\left(\bar{c}_{\beta} \sigma^{2} \boldsymbol{\sigma} \bar{d}_{\alpha}\right)\right]-\left(\sigma^{2} \leftrightarrow \sigma^{2} \boldsymbol{\sigma}\right)= \\
& =-\left[\left(c \sigma^{2} \bar{c}\right)\left(u \sigma^{2} \boldsymbol{\sigma} \bar{d}\right)-\left(c \sigma^{2} \boldsymbol{\sigma} \bar{c}\right)\left(u \sigma^{2} \bar{d}\right)\right]-i\left(c \sigma^{2} \boldsymbol{\sigma} \bar{d}\right) \times\left(u \sigma^{2} \boldsymbol{\sigma} \bar{c}\right) \\
& \sim \eta_{c} \rho^{+}-\psi \pi^{+}+i \bar{D}^{* 0} \times D^{*+}
\end{aligned}
$$

to be compared with the difference between the first two rows in (5.23), and

$$
\begin{aligned}
Z^{++} & =i\left(c^{\alpha} \sigma^{2} \boldsymbol{\sigma} u^{\beta}\right) \times\left[\left(\bar{c}_{\alpha} \sigma^{2} \boldsymbol{\sigma} \bar{d}_{\beta}\right)-\left(\bar{c}_{\beta} \sigma^{2} \boldsymbol{\sigma} \bar{d}_{\alpha}\right)\right]= \\
& =-\left[\left(c \sigma^{2} \bar{c}\right)\left(u \sigma^{2} \boldsymbol{\sigma} \bar{d}\right)+\left(c \sigma^{2} \boldsymbol{\sigma} \bar{c}\right)\left(u \sigma^{2} \bar{d}\right)\right]+\left[\left(c \sigma^{2} \bar{d}\right)\left(u \sigma^{2} \boldsymbol{\sigma} \bar{c}\right)+\left(c \sigma^{2} \boldsymbol{\sigma} \bar{d}\right)\left(u \sigma^{2} \bar{c}\right)\right] \\
& \sim \eta_{c} \rho^{+}+\psi \pi^{+}-\left(\bar{D}^{0} D^{*+}+D^{+} \bar{D}^{* 0}\right)
\end{aligned}
$$

from the sum of the first two rows in (5.23). Meson-meson amplitudes of the kind described in Figure 22 contain diquarkonia in both the $J^{P C}=1^{++}$and $J^{P C}=1^{+-}$eigenchannels.

As for the neutral component

$$
Z^{0} \sim \eta_{c} \rho^{0}-\psi \pi^{0}+i \bar{D}^{* 0} \times D^{* 0}-i \bar{D}^{*-} \times D^{*+}
$$

The neutral components for the $X$ are particularly interesting. We may have as well

$$
\begin{aligned}
X_{u} & =[c u]_{0}[\bar{c} \bar{u}]_{1}+[c u]_{1}[\bar{c} \bar{u}]_{0} \\
X_{d} & =[c d]_{0}[\bar{c} \bar{d}]_{1}+[c d]_{1}[\bar{c} \bar{d}]_{0}
\end{aligned}
$$

Here $C=+$. Take the $X_{d}$ for example. It may be rewritten as

$$
X_{d} \sim\left(D^{*-} D^{+}-D^{*+} D^{-}\right)+i\left(\psi \times \rho^{0}-\psi \times \omega^{0}\right)
$$

whose charge conjugation $C=+$ is obtained by by 29

$$
\begin{aligned}
& \left(c \sigma^{2} \bar{d}\right)\left(d \sigma^{2} \boldsymbol{\sigma} \bar{c}\right)-\left(c \sigma^{2} \boldsymbol{\sigma} \bar{d}\right)\left(d \sigma^{2} \bar{c}\right) \rightarrow\left(\bar{c} \sigma^{2} d\right)\left(\bar{d} \sigma^{2} \boldsymbol{\sigma} c\right)-\left(\bar{c} \sigma^{2} \boldsymbol{\sigma} d\right)\left(\bar{d} \sigma^{2} c\right) \\
& \quad=-\left(d \sigma^{2} \bar{c}\right)\left(c \sigma^{2} \boldsymbol{\sigma} \bar{d}\right)+\left(d \sigma^{2} \boldsymbol{\sigma} \bar{c}\right)\left(c \sigma^{2} \bar{d}\right)
\end{aligned}
$$

Given that $X_{d}$ contains both $I=0,1$, we include both $\omega$ and $\rho$ in Eq. 5.36. The same for the $X_{u}$ state. Exchanging $d \rightarrow u$

$$
X_{u} \sim\left(\bar{D}^{* 0} D^{0}-D^{* 0} \bar{D}^{0}\right)-i\left(\psi \times \rho^{0}+\psi \times \omega^{0}\right)
$$

In other words, should the quark loop diagram in Figure 22 develop a pole in one of the eigenchannels, the meson pairs coupled to the resonance would have precisely the right quantum numbers to arise from the color Fierz rearranged diquark-antidiquark state.

It is tempting to assume that all three channels corresponding to the antisymmetric diquark develop a pole, in which case molecules at meson-meson thresholds and tetraquarks would coincide.

\footnotetext{
${ }^{29}$ Actually, under charge conjugation $u \sigma^{2} \bar{c} \equiv u^{T} \sigma^{2} \bar{c}$ which is the non-relativistic limit of $\psi^{T} C \gamma_{5} \chi \rightarrow \psi_{c}^{T} C \gamma_{5} \chi_{c}=\psi_{c}^{T} C \gamma_{5} C^{-1} C \chi_{c}=$ $\psi_{c}^{T} \gamma_{5}^{T} C \chi_{c} \equiv\left(\psi_{c}^{T} \gamma_{5}^{T} C \chi_{c}\right)^{T}=\chi_{c}^{T} C^{T} \gamma_{5} \psi_{c}(-1)=\chi_{c}^{T} C \gamma_{5} \psi_{c}=c \sigma^{2} \bar{u}$. On the other hand proceeding like in Eq. (5.37) we would have $u \sigma^{2} \bar{c} \rightarrow$ $\bar{u} \sigma^{2} c \equiv\left(\bar{u}^{T} \sigma^{2} c\right)^{T}=-c^{T}\left(\sigma^{2}\right)^{T} \bar{u}=c^{T} \sigma^{2} \bar{u}$ corresponding to $c \sigma^{2} \bar{u}$. When considering charge conjugation in cases like $u \sigma^{2} \sigma \bar{u}$, the same techniques apply, but we also have to remember that $C \gamma_{\mu} C^{-1}=-\gamma_{\mu}^{T}$.
} 


\subsection{Further remarks}

The issue of tetraquarks in the $1 / N$ expansion has been commented thoroughly in a number of papers with different approaches, like putting all quarks in the antisymmetric representation, as done in [126] - the two representations coincide for $N=3$. What found there is that in such large- $N$ limit, a sort of extreme version of the Corrigan-Ramond scheme [130] (which would take only one of the quarks to transform according to the two-index antisymmetric representation and leaves the remaining ones in the fundamental representation), one can produce tetraquarks in a completely natural way, because new color-entangled operators exist.

In [120] it is shown that the Coleman-Witten lore that no tetraquarks occur at large- $N$, because their residue disappears at $N \rightarrow \infty$, is flawed. In particular, to evaluate the tetraquark $n$-point correlators one has to work large but finite $N$ and only at the end send $N \rightarrow \infty$. In this case, they appear as very narrow states. Therefore tetraquarks can be made the way Weinberg suggests, but with the concerns discussed in $[122,125]$. All this was of further stimulus to study if tetraquarks poles could instead be relevant at higher orders in the $1 / N$ expansion, as discussed in the previous Sections. The fact that subleading topologies may be important, as pointed here, seems to emerge also to explain the large- $N$ behavior of the lightest scalars [127].

Aside from the intrinsic interest of the topic, the present discussion should be considered as instrumental to the following section. The key points one should keep in mind are:

1. Neutral or charged tetraquarks could appear in non-planar diagrams only. For the latter ones the tetraquark contribution cannot disappear from the cut diagrams unless the order of the expansion is changed.

2. The mixing of tetraquarks with mesons and the decay constants are found to be suppressed by powers of $f \sim 1 /(N \sqrt{N})$ and $G \sim 1 / N^{2}(G \sim 1 /(N \sqrt{N}))$ for the neutral (charged) tetraquarks. This means that production, mixing with ordinary mesons, and decays of tetraquarks are quite suppressed in the large $N$ limit, and tetraquarks might appear more like glueballs, largely decoupled from the meson sector;

3. Color meson-meson amplitudes correlating hidden-charm+meson to open charm states, especially in the large $N$ limit, display diquarkonia in their eigenchannels.

In many respects what emerges from this discussion of tetraquarks in the $1 / N$ expansion reinforces the thesis that a dynamical interplay between diquarkonia and thresholds should be at work.

\section{An alternative picture of $X Z$ resonances}

In this Section we propose some arguments on an alternative explanation of the exotic hadron spectrum of $X Z$ resonances, following some early discussions appeared in $[10,11,30]$. Differently from other Sections, this one contains a higher fraction of material not discussed before and it relies on results discussed in Sections 2, 4, and 5. The mechanism we are going to describe, has been considered in different setups, for ordinary hadron spectroscopy [131], and more specifically for the $X Y Z$ states $[19,132]$.

In brief the conclusion of this Section is the following: effective interactions, rearranging the quarks in mesonmeson pairs into specific compact tetraquark states whose mass is higher than the meson-meson threshold, are enhanced when the energy of the free meson-meson pair in the continuum spectrum matches the mass of the compact tetraquark (a discrete level in a binding potential).

Not all compact tetraquarks are experimentally visible because their mixing to mesons is extremely small - as suggested by $1 / N$ expansion arguments. In our view, only those tetraquarks that receive an enhancement from the 
mechanism described below will manifest themselves in the experimental data. This also explains why not all mesonmeson thresholds have a resonance associated to it: simply there are no loosely bound meson-meson molecules in this picture.

The attempt is that of responding to the most evident problems plaguing the compact tetraquark and molecular models with a simple scheme able to describe most of the best assessed experimental states.

\subsection{The discrete spectrum of diquarkonia in the continuum of free meson-meson states}

Let us consider a system of four quarks produced in a small enough phase-space volume. The quantum state $\Psi$ describing this system can be represented as a superposition of alternative ways of neutralizing the color

$$
\Psi=\alpha \Psi_{d}+\beta \Psi_{m}
$$

where

$$
\Psi_{d}=\left(\epsilon_{i j k} Q^{j} q^{k}\right)\left(\epsilon^{i m n} \bar{Q}_{m} \bar{q}_{n}^{\prime}\right)
$$

is the diquark-antidiquark alternative and

$$
\Psi_{m}=\left(Q^{i} \bar{q}_{i}\right)\left(\bar{Q}_{k} q^{\prime k}\right) \text { or }\left(Q^{i} \bar{Q}_{i}\right)\left(\bar{q}_{k} q^{\prime k}\right)
$$

is the meson-meson alternative. There are no know superselection rules forbidding to consider such a coherent superposition.

In the Coleman-Witten picture, the compact tetraquark is equivalent to two free mesons ${ }^{30}$, therefore there should be no real distinction between $\Psi_{m}$ (two free mesons) and $\Psi_{d}$. But what we called a diquarkonium tetraquark in the previous Section, only appears at subleading non-planar orders and its mixing with mesons is rather suppressed at large- $N$. This allows to keep $\Psi_{m}$ and $\Psi_{d}$ distinguished.

Let us assume that $|\alpha| \ll|\beta|$, treating the diquarkonium state as a perturbation to the prominent free mesonmeson state. We consider the persistent non-observation of several predicted diquarkonia to be a consequence of this hypothesis.

The $\Psi_{m}$ state has energy $E_{m}$ in the continuous spectrum, with $E_{m} \geq M_{1}+M_{2}$, where $M_{1,2}$ are the masses of its free meson components. The $\Psi_{d}$ states, being bound states of colored objects, can have discrete values of the energy $E_{d}$, corresponding to the fundamental and excited diquarkonium states produced by strong interactions. Consider values of $E_{d}$ such that $E_{d} \geq M_{1}+M_{2}$.

If the $\Psi_{d}$ and $\Psi_{m}$ states are in orthogonal spaces (see Appendix C), $m \rightarrow m$ transitions with amplitudes

$$
T_{m m} \sim \frac{\left|\left\langle\Psi_{d} \mid H_{I} \Psi_{m}\right\rangle\right|^{2}}{E_{m}-E_{d}+i \epsilon}
$$

are allowed. Here we consider only that particular $E_{d}$, if any, which occurs close (from above) to the $M_{1}+M_{2}$ threshold. It corresponds to the mass of the would-be diquarkonium. The numerator, containing the effective interaction Hamiltonian $H_{I}$ in the continuous spectrum, is difficult to estimate, but expected to be small, on the basis of large- $N$ arguments. Therefore the term $T_{m m}$ is not negligible only when $E_{m} \sim E_{d}$.

Eq. (6.4) should be confronted to Eq. (2.18) noting that in the latter case the discrete level is found in the negative energy spectrum of some potential $V$ whereas in the case of (6.4) the discrete level is on the same side of the continuous

\footnotetext{
${ }^{30}$ The two-point function of a tetraquark is equivalent to the product of two free meson two-point functions.
} 
spectrum (therefore the $i \epsilon$ prescription is needed, as in the third term of Eq. (2.17)), but pertains to a different potential with respect to that giving the onset of continuum levels ${ }^{31}$.

The total rate for all reactions initiated by $\Psi_{m}$ in a volume $V$ (i.e. with a density of initial meson-meson pairs produced in hadronization $=\rho=1 / V$ ) is given by unitarity

$$
\begin{aligned}
\Gamma & =-16 \pi^{3} \rho \operatorname{Im}\left(T_{m m}\right) \simeq 16 \pi^{3} \rho \frac{\epsilon}{\left(E_{m}-M_{4 q}\right)^{2}+\epsilon^{2}}\left|\left\langle\Psi_{d} \mid H_{I} \Psi_{m}\right\rangle\right|^{2} \\
& =16 \pi^{4} \rho \delta\left(\frac{p^{2}}{2 M_{1}}+\frac{p^{2}}{2 M_{2}}-\delta\right)\left|\left\langle\Psi_{d} \mid H_{I} \Psi_{m}\right\rangle\right|^{2}
\end{aligned}
$$

where the detuning parameter $\delta=M_{4 q}-M_{1}-M_{2}$ is represented in Fig. 24 and it is assumed $|p| \ll M_{1,2}$. Hadronization randomly generates relative momenta $p$ between the components of the meson-meson system $\Psi_{m}$. The expected width $\bar{\Gamma}$ is the average of $\Gamma$ over all $\boldsymbol{p}$ 's within the ball $|\boldsymbol{p}| \lesssim \bar{p}-$ where $\bar{p}$ is the support of the diquarkonium tetraquark wavefunction in momentum space ${ }^{32}$. We consider $\bar{p}$ to be roughly less than the average radial excitation gap between diquarkonia levels.

Assuming $M_{1} \simeq M_{2} \simeq M$ we have therefore ${ }^{33}$

$$
\bar{\Gamma} \simeq A \sqrt{\delta}
$$

provided that $\sqrt{M \delta}<\bar{p}$. Here

$$
A=\frac{48 \pi^{4}}{\bar{p}^{3}} \rho\left|\left\langle\Psi_{d} \mid H_{I} \Psi_{m}\right\rangle\right|^{2} M^{3 / 2} \sim 48 \pi^{4}\left|\left\langle\Psi_{d} \mid H_{I} \Psi_{m}\right\rangle\right|^{2} M^{3 / 2}
$$

because the density of states scales like $\bar{p}^{3}$. Therefore the expected width scales with the square root of the detuning $\delta$ between the mass of the diquarkonium state (which can be estimated theoretically) and the nearest meson-meson threshold having the same quantum numbers. If $T_{i}^{j}$ is a diquarkonium tetraquark with an antidiquark of color $i$ and a diquark of color $j$, a simple ansatz for $H_{I}$ is

$$
H_{I}=B\left(T_{i}^{j} \epsilon_{j \ell m} \epsilon^{i k n} M_{k}^{\ell} M_{n}^{m}\right)
$$

i.e. an operator crossing the quarks from $T$ to the meson-meson $M M$ pair. The coupling $B$ has dimensions of $\mathrm{MeV}$ and assume that it scales as $B \sim \epsilon M, \epsilon$ being a small numerical coefficient. Meson fields and tetraquark fields might have spin. To avoid the momentum dependent transformation matrices associated with each particle spin, which arise under Lorentz transformations, one generally sums over spins to form the Lorentz invariant amplitude $E_{M_{1}} E_{M_{2}} E_{T} \sum_{\text {spins }}\left|\left\langle\Psi_{d} \mid H_{I} \Psi_{m}\right\rangle\right|^{2} \equiv R$. Therefore we have $A \sim 48 \pi^{4} \epsilon^{2}\left(M^{2} / M^{3}\right) R M^{3 / 2}$, i.e. the mass dependence in $A$ gets soften to $A \sim M^{1 / 2}$. This is computed in the meson-meson frame for sufficiently small recoils allowing to exchange energies with masses (see discussion in Section 3).

A fit to data can be done to estimate $A$ and to verify if and to which extent it is universal among the various $X, Z$ resonances - see Fig. 25.

The derivation of (6.6) is based on the assumption that

1. $\delta>0$ : the mass of the diquarkonium must be larger than the relative meson-meson threshold $-\delta<0$ corresponds to a zero rate

\footnotetext{
${ }^{31}$ This strongly differentiates our metastable state from a true resonance. The latter is above threshold but belongs to the same potential as for the two-meson state

${ }^{32}$ In the type II model the diquarks are considered to be quite distant in space (see Section 4.4). A large separation in position corresponds to a small support in momentum space.

${ }^{33}$ Similarly one finds that $\bar{\sigma}$ does not depend on $\delta$. This has the interesting consequence that we expect the $Z$ 's to be produced in prompt hadron collisions.
} 


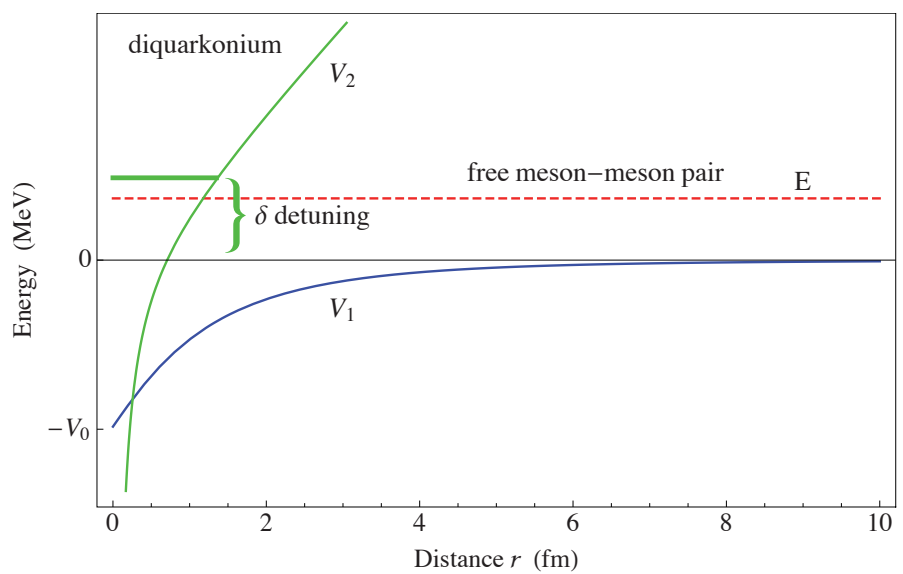

Figure 24: The short range color potential has discrete levels: the lowest lying one is shown with a thicker line. Just for the sake of illustration we qualitatively sketch a quarkonium-like potential $V_{2}$ for the radial excitations, but do not assume any explicit functional form for it. The detuning $\delta$ is the energy-gap measured from the onset of the continuous spectrum of the long-range potential to the mass of the diquarkonium state in $V_{2}$.
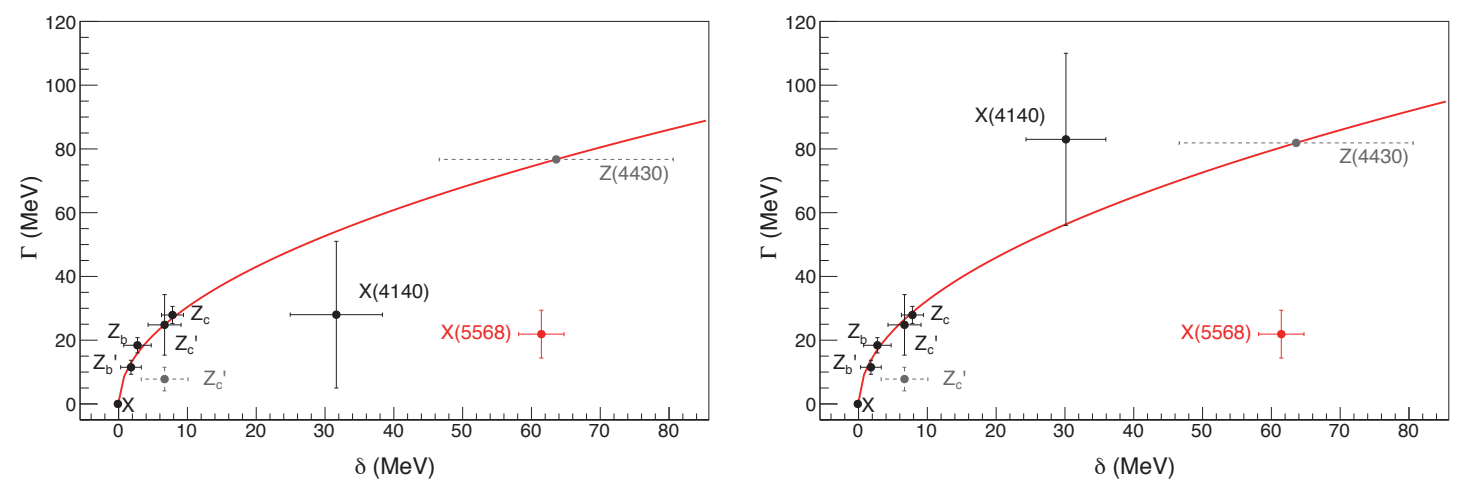

Figure 25: Width of the observed exotic mesons as a function of their detuning $\delta$ to the closest, from below, two-meson thresholds. The solid curve is the fitted function $A \sqrt{\delta}$, with $A=(10.3 \pm 1.3) \mathrm{MeV}^{1 / 2}$ with $\chi^{2} / \mathrm{DOF}=1.2 / 5$ (without the $X(4140)$, the quality of the fit would be $\left.\chi^{2} / \mathrm{DOF}=0.2 / 4\right)$. The two points associated with the $Z_{c}^{\prime}(4020)$ correspond to the two measurements of its width obtained from $\bar{D}^{* 0} D^{*+}$ (solid black) and the $h_{c} \pi$ (dashed gray) channels and which differ at $2 \sigma$ level from each other. In the fit we considered the $\Gamma\left(Z_{c}^{\prime}\right) \simeq 25 \mathrm{MeV}$ measured in the $\bar{D}^{* 0} D^{*+}$ channel, which is consistent with the width of the neutral $Z_{c}^{\prime 0}$ partner. We also show the prediction for the $Z(4430)$ width, which underestimates the total width as expected. The red point corresponds to the $X(5568)$ state whose observation has been claimed by D $\varnothing$ [133]. On the left panel we use the $X(4140)$ width as measured by CMS [134]. On the right we use the most recent LHCb value [135, 136] — see Sections 6.4 and 8.6.

2. $\sqrt{M \delta}<\bar{p}$ : the contribution of (6.4) to $\bar{\Gamma}$ comes only from small enough detunings

3. The diquarkonium state, when Fierz-transformed in terms of meson singlets, does not contain the meson components in the meson-meson pair (6.4))

With the discovery of charged $Z_{c}$ s and $Z_{b}$ s resonances, the connection to open charm/beauty meson-meson thresholds has become manifest. The $X(3872)$ mass is fine tuned with the $\bar{D}^{0} D^{* 0}$ threshold. Also the $Z_{b}(10610), Z_{b}(10650)$, $Z_{c}(3900)$ and $Z_{c}^{\prime}(4025)$ are close to the $B B^{*}, B^{*} B^{*}, D D^{*}$ and $D^{*} D^{*}$ thresholds respectively, but at energy distances of $+2.7,+1.8,+7.8,+6.7 \mathrm{MeV}$; this positive sign trend does not appear to be an accidental feature. It means that, lying above threshold values, $Z$ resonances cannot be deuteron-like states!

As illustrated in Fig. 25, the $\sqrt{\delta}$ fit works rather well for $X, Z_{b}, Z_{b}^{\prime}, Z_{c}, Z_{c}^{\prime}$, and $X(4140)$. For all of them the $\delta>0$ condition is met (in the case of $Z_{b}, Z_{b}^{\prime}, Z_{c}$, and $Z_{c}^{\prime}$, the detunings are therefore $\delta=2.7,1.8,7.8,6.7 \mathrm{MeV}$ respectively). 
The estimate of $A=(10.3 \pm 1.3) \mathrm{MeV}^{1 / 2}$ is compatible, within $2 \sigma$, with the $A \sim M^{1 / 2}$ behavior ${ }^{34}$. More will be said on condition (3) in the following.

This approach badly fails to accommodate the $X(5568)$ which was considered to falsify this approach, until its experimental disproval.

What is callled an $X, Z$ resonance could therefore result from the non-vanishing amplitude $\left\langle\Psi_{d} \mid H_{I} \Psi_{m}\right\rangle$ inducing an effective interaction in the continuous spectrum of meson-meson states due to a diquarkonium $\Psi_{d}$ located just above threshold. The free meson-meson state gets temporarily locked in the discrete level within $V_{2}$ thanks to the interaction $H_{I}$ - the diquarkonium state is produced. If $H_{I}$ were zero, the only effect in the continuous spectrum of $V_{1}$ could come from a shallow bound state, if any, at $-B$. However anything appreciable in the cross section would need a gap $E+B \approx 0$, as reported in Section 2, whereas, as we see from phenomenology, $E$ can go rather far from threshold in the continuous spectrum (the fact that $E+B$ can be as large as $E+B \approx 10 \mathrm{MeV}$, would contradict most of the hypotheses we have worked with in Section 2). In absence of a level at $-B$, and assuming $H_{I}=0$, we recall that $S$-wave (re)scattering of meson-meson pairs alone could not generate narrow width resonances.

The only fact that diquarkonium levels exist, provides an effective interaction in the meson-meson channel which allows the temporary 'hybridization' of the meson-meson system into a diquarkonium. In other words, all diquarkonium tetraquarks predicted should in principle be observed, but only few of them get produced: the conditions described above should be met. Under these conditions an enhancement of the tetraquark-meson mixing (otherwise extremely small) becomes possible.

\section{2. $\Psi_{m}$ and $\Psi_{d}$ states}

We used $\Psi_{d}$ for diquarkonium states and $\Psi_{m}$ for free meson-meson states, with negligible residual strong interactions.

Let us consider the particular case of the $Z_{c}(3900)$ resonance. From $\left(I^{G}\right) J^{P C}=\left(1^{+}\right) 1^{+-}$quantum numbers the $\Psi_{d}$ state is

$$
\Psi_{d}=[c u]_{S=0}[\bar{c} \bar{d}]_{S=1}-[c u]_{S=1}[\bar{c} \bar{d}]_{S=0}
$$

and, from (6.2), this notation is a shorthand for

$$
\Psi_{d}=\epsilon_{\alpha \beta \gamma}\left(c^{\alpha} \sigma^{2} u^{\beta}\right) \epsilon^{\alpha^{\prime} \beta^{\prime} \gamma}\left(\bar{c}_{\alpha^{\prime}} \sigma^{2} \sigma \bar{d}_{\beta^{\prime}}\right)-\left(\sigma^{2} \leftrightarrow \sigma^{2} \sigma\right)
$$

Here $\epsilon_{\alpha \beta \gamma}\left(c^{\alpha} \sigma^{2} u^{\beta}\right)$ describes a spin zero diquark whereas $\epsilon^{\alpha^{\prime} \beta^{\prime} \gamma}\left(\bar{c}_{\alpha^{\prime}} \sigma^{2} \sigma \bar{d}_{\beta^{\prime}}\right)$ has spin 1. Upon Fierz rearrangement we found

$$
\Psi_{d} \sim \eta_{c} \rho^{+}-\psi \pi^{+}+i \bar{D}^{* 0} \times D^{*+}
$$

The closer (i.e. fulfilling $\sqrt{M \delta}<\bar{p}$ ) meson-meson threshold below the measured $Z_{c}(3900)$ mass is $\bar{D}^{0} D^{*+}$, therefore the relevant threshold is

$$
\Psi_{m} \sim \bar{D}^{0} D^{*+}
$$

This is orthogonal to $\Psi_{d}$ : there is no $\bar{D}^{0} D^{*+}$ component in the $\Psi_{d}$ Fierz-transformed state. The $\Psi_{m}$ might be taken as a superposition of different meson-meson states and constructed in such a way to be orthogonal to $\Psi_{d}$. However we computed the total rate for all reactions initiated by a selected meson-meson state with fixed quantum numbers and flavor, and this is why we stick to $\Psi_{m}$ as in (6.12).

In the $Z_{c}^{\prime}$ case we found in (5.33)

$$
\Psi_{d} \sim \eta_{c} \rho^{+}+\psi \pi^{+}-\left(\bar{D}^{0} D^{*+}+D^{+} \bar{D}^{* 0}\right)
$$

\footnotetext{
${ }^{34}$ Meaning that $(10.3+2.6) /(10.3-2.6) \simeq \sqrt{M\left(Z_{b}\right) / M\left(Z_{c}\right)}$.
} 
whereas the relevant threshold must be

$$
\Psi_{m} \sim \bar{D}^{0 *} D^{*+}
$$

The case of the $Z_{b}$ s resonances is completely analogous.

In the case of the $X(3872)$ we have

$$
X_{d}=[c d]_{0}[\bar{c} \bar{d}]_{1}+[c d]_{1}[\bar{c} \bar{d}]_{0}
$$

where the subscript $d$ stands here for $d$-quark. As it was shown in Section 5.3, this latter state can be rewritten as

$$
\Psi_{d} \sim\left(D^{*-} D^{+}-D^{*+} D^{-}\right)+i\left(\psi \times \rho^{0}-\psi \times \omega^{0}\right)
$$

whereas

$$
\Psi_{m} \sim \bar{D}^{0} D^{* 0}
$$

so orthogonality between $\Psi_{m}$ and $\Psi_{d}$ is at work. Any $\Psi_{m} \sim D^{+} D^{*-}$ would be slightly heavier than the $M\left(\Psi_{d}\right)$ mass and is not to be considered here.

On the other hand there is also a $X_{u}$ component which would Fierz to neutral open charm states. We might observe that since the mechanism we consider for the formation of the observed resonances relies on the orthogonality between $\Psi_{d}$ and the open charm meson pair $D^{0} \bar{D}^{0 *}$, only the $X_{d}$ component gets involved. This would rule out the $X_{u}$ component therefore inducing isospin violations in $X(3872)$ decays, with no need of any hyperfine neutral doublet close to $3872 \mathrm{MeV}$. This point will be discussed again in Section 6.3.

From this discussion we see that

1. We do not expect a narrow resonance in proximity of every single meson-meson threshold but only free meson pairs unless a $\Psi_{d}$, with the right quantum numbers, occurs right above threshold

2. We do not expect a resonance in correspondence of every diquarkonium state: pure diquarkonia might have small probabilities to be formed on their own given $|\alpha| \ll|\beta|$ in (6.1)

\subsection{The $X_{b}$ in the beauty sector, isospin violations and the non-observation of $X^{+}$}

A pressing question is about whether we should expect a twin particle of the $X(3872)$ in the beauty sector. Also in this case, the determination of the discrete diquarkonium level should be precise enough to allow a prediction. According to (4.50) and (4.51), the twin of the $X$ in the beauty sector should be almost degenerate with the lighter of the two $Z_{b}$ s and from (4.52)

$$
M[X([b q][\bar{b} \bar{q}])] \simeq M\left[Z_{b}(10610)\right]=(10607 \pm 2) \mathrm{MeV}
$$

If the relevant threshold is $\bar{B}^{0} B^{* 0}$, in perfect analogy with the charm sector, we have

$$
M\left(B^{0}\right)+M\left(B^{* 0}\right) \simeq(10604.4 \pm 0.3) \mathrm{MeV}
$$

This alternatively tells that

1. There is a very narrow partner of the $X(3872)$ in the beauty sector if indeed the diquarkonium level is higher by few $\mathrm{MeV}$ s as from the above rough estimate

2. There is not such a resonance because the diquarkonium mass would be below the value of $10604 \mathrm{MeV}$ in (6.19)

We might prefer the second option because it is observed in the charm sector that the experimental mass difference is

$$
M\left(Z_{c}\right)-M(X) \simeq(32 \pm 3) \mathrm{MeV}
$$


whereas the diquarkonium model would predict an approximate degeneracy. This leads to infer that the twin diquarkonium level of the $X_{b}$ falls below the relevant threshold, and no such resonance will be discovered. On the other hand, in going from the mass value of the charm to that of the beauty, we could observe a sensibly smaller lift of the $Z_{b}-X_{b}$ degeneracy and consequently a narrow $X_{b}$ particle could be eventually found in $p p$ collisions.

As for the time of this writing, there are no experimental clues of an $X_{b}$ in the beauty sector located close to $10604 \mathrm{MeV}$ (see Section 8.3).

Besides the absence of a relative of the $X(3872)$ in the beauty sector, another serious problem with a naïve diquarkonium interpretation of this resonance is the absence of a almost degenerate doublet of neutral states $X_{u}=[c u][\bar{c} \bar{u}]$ and $X_{d}=[c d][\bar{c} \bar{d}]$. The need of it comes from the observation of strong isospin violation in the $X$ decays [137]

$$
\frac{\mathcal{B}(X \rightarrow J / \psi \omega)}{\mathcal{B}(X \rightarrow J / \psi \rho)} \simeq 0.8 \pm 0.3
$$

If the mass matrix were of the kind

$$
M=\left(\begin{array}{cc}
2 m_{[c q]} & 0 \\
0 & 2 m_{[c q]}
\end{array}\right)+\epsilon\left(\begin{array}{ll}
1 & 1 \\
1 & 1
\end{array}\right)
$$

it would have two eigenstates

$$
\begin{array}{lll}
\frac{|1\rangle-|2\rangle}{\sqrt{2}} & \text { with } & \lambda=2 m_{[c q]} \\
\frac{|1\rangle+|2\rangle}{\sqrt{2}} & \text { with } & \lambda=2 m_{[c q]}+2 \epsilon
\end{array}
$$

corresponding to two isospin eigenstates $I=0,1$ with approximately the same mass, $\epsilon$ being a small annihilation term $u \bar{u} \leftrightarrow d \bar{d}$. Here $|1\rangle$ and $|2\rangle$ are the basis vectors

$$
|1\rangle=\left(\begin{array}{l}
1 \\
0
\end{array}\right) \sim X_{u} \quad|2\rangle=\left(\begin{array}{l}
0 \\
1
\end{array}\right) \sim X_{d}
$$

If $\epsilon$ is negligible with respect to $m_{[c q]}$, i.e. annihilations are negligible, we can set it to zero to first approximation and this breaks isospin giving $X_{u}$ and an $X_{d}$, degenerate in mass, each maximally isospin mixed. Each of them, $X_{u}$ and $X_{d}$, can therefore decay into $J / \psi \omega$ and $J / \psi \rho$ with almost the same rate. However an hyperfine splitting between them is expected and it even seemed to be in data for some time [86] - those results were never confirmed in successive data analyses.

On the other hand, as discussed in Eqs. $(6.15,6.16)$, only the $X_{d}$ is subject to the hybridization phenomenon described. The state $X_{u}$ would instead have a projection on the $\bar{D}^{0} D^{* 0}$. Ruling out $X_{u}$ in favor of $X_{d}$ at the same time breaks isospin, as required by data, and solves the problem of the absence of an hyperfine neutral doublet at about $3872 \mathrm{MeV}$.

Finally, also the problem of the non-observation of $X^{+}$might be addressed within the same scheme. The $V_{2}$ potential gives almost degenerate $X^{+}, X^{0}$ would-be compact tetraquark levels. On the other hand the $D^{+} \bar{D}^{* 0}, \bar{D}^{0} D^{*+}$ thresholds happen to be slightly heavier than those. This might be the reason why we do not observe $X^{+}$resonances. Most remarkably the $\Psi_{d}$ state for the $X^{+}$is not orthogonal to the relevant (neutral) threshold. This is summarized in Table 7.

The $J / \psi \rho$ and $J / \psi \omega$ thresholds are sensibly above $M\left(\Psi_{d}\right)$, the mass of the diquarkonium state. This might be a reason not to include them in this discussion. However, given the width of the $\rho$ meson especially, they could have. 
Yet $J / \psi \rho$ and $J / \psi \omega$ are not included in the total width calculation in (6.5), because of (6.16): the enhancement in (6.5) is obtained for $\Psi_{d}$ and $\Psi_{m}$ orthogonal states only.

Even though $X$, in this picture, is prominently produced by $D D^{*}$, it can also decay in $J / \psi \rho(\omega)$, with a phase factor penalty, as it is clear from observations on $X^{0}$.

The case of $X(3872)$, among the one examined here, is particularly extreme because of the very narrow splittings involved.

The picture described here of a resonant hybridization with a tuned diquarkonium level in the meson-meson channel has been first discussed in [11]. Some earlier considerations on the same topic can be found in [138] and in [10, 30], although formulated differently.

\subsection{The special case of the $X(4140)$}

As discussed in Section 4.4, diquarkonium spectra can be computed assuming an interaction Hamiltonian depending on the spin degrees of freedom of quarks within diquarks [8]. From Eqs. $(4.52,4.53)$

$$
M_{Z^{\prime}}-M_{Z}=2 \kappa
$$

and

$$
M_{Z^{\prime}}+M_{Z}=4 m_{[Q q]}
$$

so that

$$
\begin{aligned}
\kappa_{c q} & \simeq 67 \mathrm{MeV} \\
m_{[c q]} & \simeq 1980 \mathrm{MeV}
\end{aligned}
$$

We argue that

$$
m_{[c s]} \simeq m_{[c q]}+\left(m_{s}-m_{q}\right) \simeq 2100 \mathrm{MeV}
$$

with $\left(m_{s}-m_{q}\right) \simeq 120 \mathrm{MeV}$ from the $\mathrm{SU}(3)$ decuplet (this mass difference, when taken from $D_{s}$ mesons, would be lighter by $\sim 20 \mathrm{MeV}$ ). It is also worth noticing that the difference in mass between $X(4140)$ and $X(3872)$ is consistent with the $\psi-\rho$ mass difference. We use the naïve scaling law to determine

$$
\kappa_{c s}=\kappa_{c q} \frac{m_{q}}{m_{s}} \simeq(45 \pm 3) \mathrm{MeV}
$$

where the quark mass are the constituent ones as taken from Table I in [7]. Therefore, from the spectrum in Figure 8 we have (see also [139])

$$
M\left(X\left(4140 ; 1^{++}\right)\right)=2 \times 2100-45=4155 \mathrm{MeV}
$$

(or as light as $4410 \mathrm{MeV}$ with a smaller mass difference) which, considering the approximations made, results in reasonable agreement $(4130 \pm 20 \mathrm{MeV})$ with the experimentally observed mass value. This estimate improves on what was found before the discovery of the $Z_{c}$ s system [85].

The $X(4140)$, also reported in Figure 25, is a $1^{++}$state, like the $X(3872)$, but in the diquarkonium picture it has quark content $[139,140]$

$$
X(4140)=[c s]_{0}[\bar{c} \bar{s}]_{1}+[c s]_{1}[\bar{c} \bar{s}]_{0}
$$

which is indicated by the experimental observation of the decay mode

$$
X(4140) \rightarrow J / \psi \phi
$$


fixing $C=+$. As in Eq. (5.36) we have

$$
\Psi_{d} \sim\left(D_{s}^{*-} D_{s}^{+}-D_{s}^{*+} D_{s}^{-}\right)-i(\psi \times \phi)
$$

On the other hand, the closest threshold from below with quark content $c s \bar{c} \bar{s}$ and $J^{P C}=1^{++}$would be $J / \psi \phi$, which is located just $20 \mathrm{MeV}$ below $4140 \mathrm{MeV}$ (about $40 \mathrm{MeV}$ higher than $D_{s}^{*-} D_{s}^{+}$). In both cases, $\Psi_{m}=D_{s}^{*-} D_{s}^{+}, \psi \times \phi$, would have a non-zero overlap with $\Psi_{d}$. This forbids the mechanism we presented here and it could be that this resonance needs some other explanation. Indeed there are other aspects making of the X(4140) a special case.

Recent results by the $\mathrm{LHCb}$ collaboration confirm it, but with a total width of about $(83 \pm 21) \mathrm{MeV}$, much larger than what reported before. Quantum numbers are confirmed to be $1^{++}$. Most importantly, three more states are observed (with slightly less significance)

$$
X\left(4274 ; 1^{++}\right), \quad X\left(4500 ; 0^{++}\right), \quad X\left(4700 ; 0^{++}\right)
$$

and similar decay modes. A second $1^{++}$state (heavier by $130 \mathrm{MeV}$ ) is not expected in the diquark-antidiquark picture as can be seen from Fig. 8 .

Heavier states might be easily considered to be radial excitations ${ }^{35}: X\left(4500 ; 0^{++}\right), X\left(4700 ; 0^{++}\right)$, could be the radial excitations of the two scalar states $0^{++}$and $0^{++\prime}$ expected by the diquarkonium model, see Eqs. (4.57) - the lowest of them cannot kinematically decay into $J / \psi \phi[141]$.

But the second $1^{++}$state remains a problem. We might suspect that the second $1^{++}$state, $X\left(4274 ; 1^{++}\right)$, has not been identified correctly: its quantum numbers are not $1^{++}$but either $0^{++}$or $2^{++}-$see Fig. 8 . Another possibility is that two almost unresolved peaks, one with $0^{++}$and the other with $2^{++}$quantum numbers, are observed at $\sim 4270 \mathrm{MeV}$. Angular information would be washed out for the sum of the two states to make it appear as one with averaged spinparity, see [140].

Another, even stronger hypothesis, is that (anti)quark-(anti)quark systems might, less likely, be produced also in the color symmetric configuration $\mathbf{6}(\overline{\mathbf{6}})$. We would not talk of diquarks in that case. Overall $\mathbf{6} \times \overline{\mathbf{6}}$ contains the color singlet and we would have $\Psi_{s}$ stationary states, in some other potential $V_{3}$ in Fig. 24. The spectrum of $\Psi_{s}$ states should be different from that of $\Psi_{d}$ ones, as computed in (6.30) - with an unknown mass gap. Yet we could assume

$$
X(4140)=\{c s\}_{0}\{\bar{c} \bar{s}\}_{1}+\{c s\}_{1}\{\bar{c} \bar{s}\}_{0}
$$

where curly brackets denote color 6 quark-quark pairs. The $\Psi_{s}$ state associated would be

$$
\Psi_{s} \sim \psi \times \phi+D_{s}^{*+} \times D_{s}^{*-}
$$

to be compared with the meson-meson threshold

$$
\Psi_{m}=D_{s}^{*-} D_{s}^{+}
$$

This would have the interesting consequence to make the mechanism here described possible, and force to use the detuning $\delta$ to the $D_{s}^{*-} D_{s}^{+}$threshold. This predicts $\bar{\Gamma} \sim 10 \times \sqrt{60} \sim 77 \mathrm{MeV}$, to be compared with the LHCb value $\Gamma_{\exp }=(83 \pm 21) \mathrm{MeV}$. On the other hand this would not solve the problem of having two observed $1^{++}$states - a spin misedintification is still the favored option. In the diquarkonium tetraquark picture [142], this gets solved doubling the spectrum with $\overline{\mathbf{3}}$ diquarks and $\mathbf{6}$ quark-quark pairs.

\subsection{Summary}

In Table 7, for each of the known multiquark resonances, we report whether or not they fulfill the criteria to be produced with the mechanism described in this Section.

\footnotetext{
${ }^{35}$ Although all these states appear to decay into $J / \psi$ rather that $\psi(2 S)$ (in contrast to the $Z(4430)$, which decays prominently into $\left.\psi(2 S) \pi\right)$, and the mass gap looks too small with respect to the $M(Z(4430))-M\left(Z_{c}(3900)\right)$ one.
} 


\begin{tabular}{|c|c|c|c|c|c|}
\hline Resonance & $\Psi_{m}$ & $\Psi_{d}$ & $M\left(\Psi_{d}\right) \geq M\left(\Psi_{m}\right)$ & $\Psi_{d} \perp \Psi_{m}$ & Exp \\
\hline$X_{u}^{0}$ & $\bar{D}^{0} D^{* 0}$ & $\begin{array}{c}\left(\bar{D}^{* 0} D^{0}-D^{* 0} \bar{D}^{0}\right) \\
-i \psi \times\left(\rho^{0}+\omega^{0}\right)\end{array}$ & yes & no & $x$ \\
\hline$X_{d}^{0}$ & $\bar{D}^{0} D^{* 0}$ & $\begin{array}{l}\left(D^{*-} D^{+}-D^{*+} D^{-}\right) \\
+i \psi \times\left(\rho^{0}-\omega^{0}\right)\end{array}$ & yes & yes & $\sqrt{ }$ \\
\hline$X^{+}$ & $D^{+} \bar{D}^{* 0} \vee \bar{D}^{0} D^{*+}$ & $\left(D^{+} \bar{D}^{* 0}-\bar{D}^{0} D^{*+}\right)-i \psi \times \rho^{+}$ & no & no & $x$ \\
\hline$Z_{c}^{0}$ & $\bar{D}^{0} D^{* 0}$ & $\begin{array}{c}\eta_{c} \rho^{0}-\psi \pi^{0} \\
+i \bar{D}^{* 0} \times D^{* 0}-i \bar{D}^{*-} \times D^{*+}\end{array}$ & yes & yes & $\sqrt{ }$ \\
\hline$Z_{c}^{+}$ & $\bar{D}^{0} D^{*+}$ & $\eta_{c} \rho^{+}-\psi \pi^{+}+i \bar{D}^{* 0} \times D^{*+}$ & yes & yes & $\sqrt{ }$ \\
\hline$Z_{c}^{\prime 0}$ & $\bar{D}^{* 0} D^{* 0}$ & $\begin{array}{c}\eta_{c} \rho^{0}+\psi \pi^{0}+\left(\bar{D}^{0} D^{* 0}+D^{0} \bar{D}^{* 0}\right) \\
-\left(D^{-} D^{*+}+D^{+} D^{*-}\right)\end{array}$ & yes & yes & $\checkmark$ \\
\hline$Z_{c}^{\prime+}$ & $\bar{D}^{* 0} D^{*+}$ & $\left(\eta_{c} \rho^{+}+\psi \pi^{+}\right)-\left(\bar{D}^{0} D^{*+}+D^{+} \bar{D}^{* 0}\right)$ & yes & yes & $\sqrt{ }$ \\
\hline$\left(X_{b}^{0}\right)_{u}$ & $\bar{B}^{0} B^{* 0}$ & $\begin{array}{l}\left(\bar{B}^{* 0} B^{0}-B^{* 0} \bar{B}^{0}\right) \\
-i \Upsilon \times\left(\rho^{0}+\omega^{0}\right)\end{array}$ & no & no & $x$ \\
\hline$\left(X_{b}^{0}\right)_{d}$ & $\bar{B}^{0} B^{* 0}$ & $\begin{array}{l}\left(B^{*-} B^{+}-B^{*+} B^{-}\right) \\
+i \Upsilon \times\left(\rho^{0}-\omega^{0}\right)\end{array}$ & no & yes & $x$ \\
\hline$X_{b}^{+}$ & $B^{+} \bar{B}^{* 0} \vee \bar{B}^{0} B^{*+}$ & $\left(B^{+} \bar{B}^{* 0}-\bar{B}^{0} B^{*+}\right)-i \Upsilon \times \rho^{+}$ & no & no & $x$ \\
\hline$Z_{b}^{(\prime) 0}, Z_{b}^{(\prime)+}$ & like $Z_{c} \mathrm{~s}$ & like $Z_{c} \mathrm{~s}$ & yes & yes & $\sqrt{ }$ \\
\hline
\end{tabular}

Table 7: We resume here the mechanism described. In the last column to the right the final evaluation: is the resonance expected in data (checkmark) or not (cross)? Some relevant features: $i$ ) there is only one neutral $X$ which decays violating isospin - this is not the case for neutral $Z_{c} \mathrm{~s}$ or $Z_{b} \mathrm{~s} i$ i) there are no neutral $X \mathrm{~s}$ in the beauty sector — one of the two states is subject to the approximate evaluation of the diquarkonium mass though $\mathrm{iii}$ ) there are no charged $X$ in the charm nor in the beauty sector.

The starting point of our discussion is that the all the observed XZ states appear to be above threshold. We are not considering this as an accidental pattern. Being the detunings fairly small, different parametrizations of the lineshapes might move the states below threshold $[143,144]$. Forthcoming data will be able to resolve the proper lineshapes and give a definitive answer to this question. For the time being, we decide to trust the results currently provided by the experiments, and take $\delta>0$.

If on the other hand the $X^{+}(\sim 3872)$ will eventually be discovered, suppose with a larger width than $X^{0}$ for example, or $X_{b}$ in the beauty, or any other among the states predicted by the diquarkonium model, that would challenge or simply invalidate the given description in favor of the diquarkonium tetraquark model, or, depending on the results, in terms of other approaches.

Secondly, it is extremely plausible that compact tetraquarks are indeed part of the QCD spectrum, as understood also on the basis of large- $N$ QCD considerations. Therefore, one might expect to observe them even in absence of close by meson-meson thresholds. Our assumption is that, unless enhanced by the mechanism described, the probability of producing a diquarkonium tetraquark on his own is very small. Exception made for large- $N$ QCD, we do not have a more rigorous explanation, from first principles, of this point, but we rather consider it mostly motivated, a posteriori, 
by the persisting experimental lack of several diquarkonium states ${ }^{36}$.

It is not clear yet how to treat those resonances that could be formed in correspondence of radial excitations of diquarkonia because we are not sure about how the mechanism works, and if it does, in the case in which transitions are possible within the closed channel (in the potential $V_{2}$ of Figure 24). Also neutral $J^{P C}=1^{--} Y$ resonances, identified as orbital excitations of diquarkonia (Section 4.6), have not been included in this discussion for the same reason.

\section{Other models and approaches}

In this Section we illustrate other options for the description of $X Y Z$ phenomenology. These were proposed since the early days after the discovery of the $X(3872)$ and the $Y(4260)$ and might have a somewhat more restricted application since they do not apply 'systematically' to all of the observed states. The fact that $X Y Z$ observed resonances should all be of the same nature contains admittedly some prejudice, which motivated many of the arguments made in this review. Until a widely accepted picture is not reached, we have to consider the possibility that not all of them can be understood with the same scheme.

\subsection{Hadroquarkonium}

The peculiar property of the vector $Y$ states and of the $Z(4430)$ motivated their interpretation in terms of another possible hadron configuration, the so-called hadroquarkonium [145-148]. These states decay predominantly into a particular charmonium + light mesons, $J / \psi$ for the $Y(4260)$ and the $Z_{c}(3900)$ and $\psi(2 S)$ for the others. In particular, they are not observed decaying into open charm final states, despite these modes are expected to dominate the width in all ordinary charmonium models. Therefore, one of their possible interpretations is that of a 'core' composed by a compact $Q \bar{Q}$ state, surrounded by a "cloud" of light hadronic matter. While meson molecules (Section 3) and diquarkonia (Section 4) are discriminated by the overlapping of their constituents - largely separated mesons for the former and compact diquarkonia for the latter - hadroquarkonia would be characterized by the relative sizes of the core and the cloud. The light matter cloud is thought to have a typical radius much larger than that of the central quarkonium.

The hadroquarkonium model shares several qualitative features with ordinary atomic physics. The potential between its two components would be a QCD analogous of the van der Waals force. The key assumption is that such interaction is strong enough to allow for a bound state but not enough to substantially modify the nature of the compact $Q \bar{Q}$ core. If this is verified, then the decay pattern of the above mentioned resonances would be simply explained. However, as we will see shortly, it is difficult to reach a full assessment of these hypotheses.

An analysis to look for hadroquarkonia on the Lattice has been presented in [149].

\subsubsection{The Hamiltonian}

Since the $Q \bar{Q}$ core is a color neutral object, its interaction with the gluonic field generated by the light degrees of freedom can be treated using a QCD multipole expansion [150]. The effective Hamiltonian for such an interaction can be written as [150-153]

$$
H_{\mathrm{eff}}=-\frac{1}{2} \alpha^{\left(\psi_{1} \psi_{2}\right)} E_{i}^{a} E_{i}^{a}
$$

\footnotetext{
${ }^{36}$ On the other hand, the absence of meson molecules from the ensemble of observed states is clearly explained by the production issues reported in Sections 3.3 and Appendix A.
} 
We adopt the Wilsonian normalization of the gluonic fields ${ }^{37}$. The previous equation is simple to understand in analogy with the electromagnetic case. The leading interaction between an overall neutral composite object and an external electric field is proportional to $-\boldsymbol{d} \cdot \boldsymbol{E}$, with $\boldsymbol{d}$ the dipole moment of the object. At the same time, the induced dipole must be proportional to the external field itself, $\boldsymbol{d} \propto \alpha \boldsymbol{E}$, with $\alpha$ being the polarizability. Therefore, the chromopolarizability $\alpha^{\left(\psi_{1} \psi_{2}\right)}$ is essentially a measure of the deformability of the central $Q \bar{Q}$. A less heuristic derivation can be found in Appendix D.

Very little is know ab initio about the chromo-polarizability ${ }^{38}$. The only two quantities that have been estimated are the off-diagonal elements $\alpha^{(J / \psi \psi(2 S))}$ and $\alpha^{(\Upsilon \Upsilon(2 S))}$, which have been measured from the $\psi(2 S) \rightarrow J / \psi \pi^{+} \pi^{-}$and $\Upsilon(2 S) \rightarrow \Upsilon \pi^{+} \pi^{-}$decays obtaining [154]

$$
\left|\alpha^{(J / \psi \psi(2 S))}\right| \simeq 2.00 \mathrm{GeV}^{-3} \quad \text { and } \quad\left|\alpha^{(\Upsilon \Upsilon(2 S))}\right| \simeq 0.66 \mathrm{GeV}^{-3}
$$

However, in light of the definition (D.9), the chromo-polarizability should also satisfy the Schwartz inequality

$$
\alpha^{(\psi \psi)} \alpha^{\left(\psi^{\prime} \psi^{\prime}\right)} \geq\left|\alpha^{\left(\psi \psi^{\prime}\right)}\right|^{2}
$$

with $\psi=(J / \psi, \Upsilon)$ and $\psi^{\prime}=(\psi(2 S), \Upsilon(2 S))$. It is also generally expected for each diagonal term to be larger than the off-diagonal one [145]. It is natural to expect for the QCD Van der Waals force to deform the core rather than change its nature.

A first challenge to this picture comes from the $Y(4260) \rightarrow Z_{c}(3900) \pi \rightarrow(J / \psi \pi) \pi$ decay observed both at Belle [155] and BES III [156]. According to the description above, the $Y(4260)$ would be a hadrocharmonium with a $J / \psi$ core surrounded by an isoscalar pion cloud. In this context, it is hard to understand why a decay into its constituents should happen via an intermediate isovector state as the $Z_{c}$ [148]. This issue could be overcome if the $Z_{c}(3900)$ itself has a hadrocharmium structure, with the same heavy quark core. This interpretation might be motivated by the suppression of the $Z_{c} \rightarrow h_{c} \pi$ decay, which was unexpected both in the molecular and in the tetraquark models. Nevertheless, as we will see shortly, it is seriously put in jeopardy by the observation of the $Z_{c} \rightarrow D D^{*}$ decay.

Even though suppressed, the chromoelectric field generated by the hadronic cloud might also induce a transition from the core quarkonium to another, $\psi_{1} \rightarrow \psi_{2}$, with rate mostly determined by the off-diagonal chromo-polarizability $\alpha^{\left(\psi_{1} \psi_{2}\right)}$. Consequently, small but detectable decay rates into quarkonia different from the original core are expected.

Decays into open flavor mesons should be suppressed as well, since they require the splitting of the $Q \bar{Q}$ by means of the soft gluons generated by the surrounding cloud. Indeed, using the holographic soft wall model for QCD [157], it has been argued that the decay of the hadroquarkonium into open flavor mesons is exponentially suppressed by the mass of the heavy quark, $\exp \left(-b \sqrt{M_{Q} / \Lambda_{Q C D}}\right)$, with $b$ being a constant.

Although in good agreement with the $Y$ states and with the $Z(4430)$, this result challenges the interpretation of the $Z_{c}(3900)$ in terms of hadrocharmonium [158], since a prominent decay into $D D^{*}$ has been observed [159].

\subsubsection{To bind, or not to bind...}

Let us now determine under which conditions the interaction (7.1) can allow bound states. It is easy to show that the potential is indeed attractive. Neglecting light quark masses, the trace of the Yang-Mills stress-energy tensor can be written as

$$
T_{\mu}^{\mu}=\frac{\beta(g)}{2 g^{3}} F_{\mu \nu}^{a} F^{a \mu \nu}=-\frac{9}{32 \pi^{2}} F_{\mu \nu}^{a} F^{a \mu \nu}=\frac{9}{16 \pi^{2}}\left(E_{i}^{a} E_{i}^{a}-B_{i}^{a} B_{i}^{a}\right)
$$

\footnotetext{
${ }^{37}$ This means that the lagrangian is normalized as $\mathcal{L}=-1 / 4 g^{2} F_{\mu \nu}^{a} F^{a \mu \nu}$.

${ }^{38}$ It has been shown in [154] that the diagonal element $\alpha^{(\psi \psi)}$ for $\psi=J / \psi, \Upsilon$ can be measured directly through the $\psi \rightarrow \pi \pi \ell^{+} \ell^{-}$process.
} 
where we used the one-loop expression for the beta function $\beta=\left(-\frac{11}{3} N+\frac{2}{3} n_{f}\right) g^{3} /(4 \pi)^{2}$ for 3 light flavors. and $\boldsymbol{B}^{a}$ is the chromo-magnetic field. At $q=0$, its average over a state $|\mathcal{X}\rangle$ of light hadronic matter is

$$
\left\langle X\left|T_{\mu}^{\mu}(\boldsymbol{q}=0)\right| X\right\rangle=M_{X}
$$

assuming a normalization $\langle\mathcal{X} \mid \mathcal{X}\rangle=1$. The potential acting on the light matter by the static pair is obtained considering the diagonal interaction $H_{\mathrm{eff}}^{\psi \psi}$ :

$$
\mathcal{V}\left\langle X\left|H_{\mathrm{eff}}\right| X\right\rangle=-\frac{\mathcal{V}}{2} \alpha^{(\psi \psi)}\left\langle X\left|E_{i}^{a} E_{i}^{a}\right| \mathcal{X}\right\rangle=-\frac{8 \pi^{2}}{9} \alpha^{(\psi \psi)} M_{X}-\frac{\mathcal{V}}{2} \alpha^{(\psi \psi)}\left\langle X\left|B_{i}^{a} B_{i}^{a}\right| \mathcal{X}\right\rangle-\frac{8 \pi^{2}}{9} \alpha^{(\psi \psi)} M_{X}
$$

where $\mathcal{V}$ is a volume factor. Now, just like we did in Section 3.6.1, the matrix element of $H_{\text {eff }}$ provides the potential in momentum space, here also in the static limit. Therefore

$$
\mathcal{V}\left\langle\mathcal{X}\left|H_{\mathrm{eff}}\right| X\right\rangle=V(\boldsymbol{q}=0)=\int d^{3} x V(\boldsymbol{x}) \leq-\frac{8 \pi^{2}}{9} \alpha^{(\psi \psi)} M_{X}
$$

This shows that the interaction is of attractive nature. It is now a matter of understanding under which conditions it also allows for bound states. This will turn out to be a much more subtle issue.

The kinetic energy of the system can be written in a non-relativistic fashion as $T \sim p^{2} / 2 \mu \sim 1 /\left(R_{X}^{2} \mu\right)$, where $R_{X}$ is the characteristic size of the light cloud and $\mu=M_{\mathcal{X}} M_{\psi} /\left(M_{X}+M_{\psi}\right)$ is the reduced mass of the quarkonium + light matter system. Its integral will therefore be $\sim R_{X} / \mu$. We also used the fact that the momentum will typically be $p \sim 1 / R_{\mathcal{X}}$. It then follows that the total energy will be

$$
E=T+V \sim \frac{R_{X}}{\mu}+\int d^{3} x V(x) \leq \frac{R_{X}}{\mu}-\frac{8 \pi^{2}}{9} \alpha^{(\psi \psi)} M_{\mathcal{X}}
$$

Requiring this to be negative gives the following constraint for the presence of bound states

$$
\alpha^{(\psi \psi)} \frac{M_{\mathcal{X}} \mu}{R_{\mathcal{X}}} \geq C
$$

where $C$ is some constant of order one, which will eventually depend on the details of the system and on the actual definition of the characteristic size.

The previous inequality is more easily satisfied for larger values of the chromo-polarizability. Being excited quarkonia more extended than the ground state, it is extremely reasonable to expect their diagonal chromo-polarizability to be larger than that of the ground state, $\alpha^{(\psi(n S) \psi(n S))}>\alpha^{(\psi \psi)}$. This could explain why most of the resonances mentioned at the beginning of the section decay into $\psi(2 S)$ rather than $J / \psi$.

One might also argue [145] that if $R_{X}$ grows slower than $M_{X}$ then a bound state will necessarily appear for massive enough light excitations. However, the models which accurately reproduce the linear Regge trajectories - see e.g. [157] - usually find $R_{X} \propto M_{X}$. The conclusions about the possible existence of bound states are therefore substantially weakened since they crucially depend on the precise knowledge of $C$ and $\alpha^{(\psi \psi)}$, which are hardly accessible quantities.

Nevertheless, if one assumes the existence of such state, then a full zoology of them is expected. Not only they could contain different central $Q \bar{Q}$ cores, e.g. $\chi_{c J}, \eta_{c}, \eta_{c}(2 S)$ and $h_{c}$ in the charm sector ${ }^{39}$, but the light excitation $|X\rangle$ could even be baryonic, giving rise to a so-called baryo-quarkonium. The $P_{c}$ pentaquarks might be good candidates for these [160].

\footnotetext{
${ }^{39}$ HQSS also implies that hadroquarkonia having cores belonging to the same spin multiplet should be almost degenerate [66].
} 


\subsubsection{A case for heavy HQSS violation}

So far there is no evidence for possible states containing $\eta_{c}$ and $\eta_{c}(2 S)^{40}$. However, BES III has observed a large cross section for $e^{+} e^{-} \rightarrow h_{c} \pi \pi$, comparable with the $\rightarrow Y(4260) \rightarrow J / \psi \pi \pi$ one $[112,113,161]$. Although the resonant content is not clear (see Section 8.4), a relevant component for $Y(4260)$ and $Y(4260) \rightarrow h_{c} \pi \pi$ is allowed. If these decays were confirmed, they would imply a strong violation of the HQSS (the $h_{c}$ is a heavy spin singlet) and challenge the interpretation of the $Y(4260)$ and $Y(4360)$ as hadrocharmonia made of a $J / \psi$ and $\psi(2 S)$ core, respectively.

A possible solution has been proposed in [147]. The two states are considered as a mixture of both $S_{c \bar{c}}=0$ and $S_{c \bar{c}}=1$ hadrocharmonia

$$
|Y(4260)\rangle=\cos \theta\left|\Psi_{1}\right\rangle-\sin \theta\left|\Psi_{0}\right\rangle \quad \text { and } \quad|Y(4360)\rangle=\sin \theta\left|\Psi_{1}\right\rangle+\cos \theta\left|\Psi_{0}\right\rangle
$$

where $\left|\Psi_{S_{c \bar{c}}}\right\rangle$ is the hadrocharmonium state ${ }^{41}$ and $\theta$ is the mixing angle.

This hypothesis allows for interesting predictions for the pattern of $e^{+} e^{-}$annihilation into $J / \psi \pi \pi, \psi(2 S) \pi \pi$ and $h_{c} \pi \pi$ final states [147]. The electromagnetic current can only create the $\left|\Psi_{1}\right\rangle$ state and hence while the $J / \psi \pi \pi$ and $\psi(2 S) \pi \pi$ channels can be produced directly, the $h_{c} \pi \pi$ one needs to happen through the $\Psi_{1}-\Psi_{0}$ mixing. The authors also estimate the mixing angle to be large, $\theta \simeq 40^{\circ}$.

Note, however, that such a large mixing implies that the two states $\left|\Psi_{1}\right\rangle$ and $\left|\Psi_{0}\right\rangle$ are almost degenerate. Their masses are in fact given by

$$
\begin{aligned}
& M_{1}=\left\langle\Psi_{1}|H| \Psi_{1}\right\rangle=\cos ^{2} \theta M(Y(4260))+\sin ^{2} \theta M(Y(4360)) \simeq 4293 \mathrm{MeV} \\
& M_{2}=\left\langle\Psi_{0}|H| \Psi_{0}\right\rangle=\cos ^{2} \theta M(Y(4360))+\sin ^{2} \theta M(Y(4260)) \simeq 4311 \mathrm{MeV}
\end{aligned}
$$

where $H$ is the unknown Hamiltonian determining the energy of the system. The closeness of these values prevents from interpreting $\Psi_{1}$ and $\Psi_{0}$ as hadrocharmonia having a $J / \psi$ and $h_{c}$ core respectively, since their masses are more than $400 \mathrm{MeV}$ apart. Their $Q \bar{Q}$ centers might instead correspond to $\psi(2 S)$ and $h_{c}$ respectively. The decay into $J / \psi$ would happen via the $\psi(2 S) \rightarrow J / \psi$ transition [66]. This is in contrast with the first picture proposed in [145], where the $Y(4260)$ was thought of as having a $J / \psi$ core. As explained at the beginning of the section, the QCD van der Waals force should induce the decay into a charmonium different from the core with a suppressed rate. Indeed, for the $Y(4360)$ we have $\mathcal{B}\left(Y(4360) \rightarrow J / \psi \pi^{+} \pi^{-}\right) / \mathcal{B}\left(Y(4360) \rightarrow \psi(2 S) \pi^{+} \pi^{-}\right)<3.4 \times 10^{-3}-$ see Sec. 8.4. Since in Eq. (7.10) the weights for the $\Psi_{1}$ component are comparable in the two states, one would expect a similar ratio for the $Y(4260)$ as well. This is not supported by data since its decay into $\psi(2 S)$ has never been observed. One might argue that this is because the process has a fairly low $Q$-value. Nevertheless, this should imply the $Y(4260)$ to be narrower than the $Y(4360)$.

\subsection{Hybrids}

The constituent quark model allows to consider static gluons as additional degrees of freedom belonging to the adjoint representation $\mathbf{8}_{c}$ of the color group. The first consequence is the presence in the QCD spectrum of the socalled glueballs, i.e. bound states of gluons only. Indeed a product of an arbitrary number of adjoint fields always contains a color singlet, $\mathbf{8}_{c} \otimes \cdots \otimes \mathbf{8}_{c}=\mathbf{1}_{c} \oplus \ldots{ }^{42}$. The presence of glueballs has been confirmed by several Lattice QCD analyses - see .g. [163-166].

\footnotetext{
${ }^{40}$ But the $Z_{1}(4050)$ and $Z_{2}(4250)$ seen by Belle in $\chi_{c 1} \pi$ might be good candidate for hadrocharmonia.

${ }^{41}$ The $J^{P C}=1^{++}$quantum numbers can arise from an $S_{c \bar{c}}=0$ hadrocharmonium only thanks to the light degrees of freedom.

${ }^{42}$ One expects massive glueballs also in pure Yang-Mills theory without matter fields. This make them particularly interesting from a more fundamental point of view. For a review, see [162].
} 
Another intriguing possibility is that of joining together a $q \bar{q}$ in the octet representation and a constituent gluon

$$
\mathbf{3}_{c} \otimes \overline{\mathbf{3}}_{c} \otimes \mathbf{8}_{c}=\left(\mathbf{8}_{c} \oplus \mathbf{1}_{c}\right) \otimes \mathbf{8}_{c}=\mathbf{2 7}_{c} \oplus \overline{\mathbf{1 0}}_{c} \oplus \mathbf{1 0}_{c} \oplus \mathbf{8}_{c} \oplus \mathbf{8}_{c} \oplus \mathbf{8}_{c} \oplus \mathbf{1}_{c}
$$

These particles are generally referred to as hybrid mesons. They were studied in the context of a constituent quark model [167-172], of the MIT bag model, both for the light [173] and heavy sector [174], or within an effective field theory approach [175]. In the constituent models, if the angular momentum of the gluon around the $q \bar{q}$ pair is $L_{g}$, the orbital angular momentum of the $q \bar{q}$ pair $L_{q \bar{q}}$ and its spin is $S_{q \bar{q}}$, then the parity and charge conjugation of the hybrid are

$$
P=(-1)^{L_{g}+L_{q \bar{q}}} \quad \text { and } \quad C=(-1)^{L_{q \bar{q}}+S_{q \bar{q}}+1}
$$

The lightest of these particles has $L_{g}=0$. For example, we can achieve exotic quantum numbers such as $J^{P C}=1^{-+}$ through a $q \bar{q}$ pair with $L_{q \bar{q}}=1$ and $S_{q \bar{q}}=0$.

\subsubsection{Modeling the hybrids: flux tube}

The constituent quark model is just one of several ways of describing hybrid mesons. The interaction between the quark, the antiquark and the gluon can be most easily understood via the phenomenological flux tube picture. It is well-known that at large separations, the $q \bar{q}$ pair is confined in an approximately thin cylindrical region of color fields. One can attempt to describe this configuration of the QCD gauge fields using an effective string theory approach. In particular, assuming a Nambu-Goto action, ${ }^{43}$ the potential at large relative distances, $r$, is found to be $[176,177]$ (see also [178] for a textbook treatment).

$$
V_{n}(r)=\sqrt{\sigma^{2} r^{2}+\frac{\pi \sigma}{6}(12 n-1)}
$$

Here $\sigma$ is the string tension and $n$ is the radial quantum number of the string modes.

For the $n=0$ string ground state and for large $r$ one obtains the well known $V_{0}(r)=\sigma r^{44}$, typical of a confining theory, and used in different phenomenological parametrizations for describing the quarkonia spectrum (see e.g. the Cornell potential [181]). Higher values of $n$ correspond to quantized excitations of the string between the quarks and hence to hybrid mesons.

It should be stressed that, despite being easily visualized, the description of the color flux tube in terms of effective string theory is problematic. Not only its quantization in four dimensions presents issues, but the energies of the gluonic excitations are not in agreement with the non-perturbative results obtained from Lattice QCD.

An approximation often used to determine the $Q \bar{Q}$ potential for the case of heavy quarks is that of the timehonored Born-Oppenheimer (BO) approximation. It is allowed since, in the large $m_{Q}$ limit, the typical time scale of the dynamics of the light degrees of freedom is much smaller than that of the heavy ones. The $Q \bar{Q}$ pair is treated as static at some fixed distance $r$ (now considered as a parameter) and the Schrödinger equation is solved for the gluonic

\footnotetext{
${ }^{43}$ The Nambu-Goto (NG) action for a relativistic string is proportional to the area of the string itself. If $\tau$ and $\lambda$ are the proper time and the intrinsic coordinate along the string, and $X_{\mu}(\tau, \lambda)$ the spacetime position of its line element, then the NG action is given by

$$
S_{N G} \propto \int d \lambda d \tau \sqrt{-\operatorname{det}\left(\partial_{\alpha} X_{\mu} \partial_{\beta} X^{\mu}\right)}
$$

${ }^{44}$ Still for $n=0$, the first $1 / r$ correction is $\pi / 12 r$, the so-called Lüscher term [179]. This behavior has been confirmed by lattice calculations with remarkable precision [180].
} 
degrees of freedom with energy $E_{\alpha}(r), \alpha$ being the set of quantum numbers describing the gluon state. Alternatively, the nonperturbative static potential can be directly measured on the lattice. $E_{\alpha}(r)=V_{\alpha}(r)$ is then used as the effective potential for the heavy quark pair. Once the potential has been determined it is possible to look for bound states. If the static quark pair is in a color singlet configuration, one gets the usual quarkonium spectrum, while if the pair is in a color octet, one gets the hybrid spectrum.

This approach has been applied together with the previous flux tube model for the gluonic degrees of freedom in several papers, see e.g. [182-184]. Some preliminary studies performed in order to relax the BO approximation were done in [185], while the analysis of energy levels fully beyond it has been done in [186]. The authors found that the spectrum of the lowest hybrid mesons is robust. Some searches in this direction has been performed in Lattice QCD - see Section 7.5. Note that in this model the first excitation of the flux tube has quantum numbers $1^{+-}$or $1^{-+}$, in contrast with the usual $1^{--}$of the gluons. Therefore, a meson with exotic signature $1^{-+}$is obtained when the quark pair has $S_{q \bar{q}}=1$. This suggest that photoproduction can be the best production modes for these states.

\subsubsection{Modeling the hybrids: constituent gluons in Coulomb gauge}

Another possibility is to describe the hybrid mesons using the Hamiltonian QCD approach in Coulomb gauge, and treating the gluonic modes as quasi-particles moving in a non-perturbative QCD vacuum [187-190]. The main idea is to work in Coulomb gauge, $\boldsymbol{\nabla} \cdot \boldsymbol{A}^{a}=0$, so that the dynamical gluons can be separated from the instantaneous Coulomb-type forces that act between color charges. A rigorous derivation of the Coulomb gauge QCD Hamiltonian can be found in [191]. The QCD spectrum is formally found by solving the Schrödinger equation

$$
H_{\mathrm{QCD}}\left[\boldsymbol{A}^{a}, \boldsymbol{\Pi}^{a}\right] \psi_{n}\left[\boldsymbol{A}^{a}\right]=E_{n} \psi_{n}\left[\boldsymbol{A}^{a}\right]
$$

with $\Pi^{a}(x)=-i \partial / \partial \boldsymbol{A}^{a}(x)$.

The peculiar feature of this model is that the constituent gluon mass is generated dynamically. To solve Eq. (7.16) one typically makes an ansatz for the BCS vacuum wave functional as [189]

$$
\psi_{0}\left[\boldsymbol{A}^{a}\right]=\left\langle\boldsymbol{A}^{a} \mid \Omega\right\rangle=\exp \left[-\int \frac{d^{3} q}{(2 \pi)^{3}} \boldsymbol{A}^{a}(\boldsymbol{q}) \omega(q) \boldsymbol{A}^{a}(-\boldsymbol{q})\right]
$$

This represents the non-trivial gluon field distribution in the vacuum. The parameter $\omega(q)$ can be determined variationally by minimizing the vacuum expectation value (VEV) of the Hamiltonian, $\partial\left\langle\psi_{0}\left|H_{\mathrm{QCD}}\right| \psi_{0}\right\rangle / \partial \omega(q)=0$. The solution is typically well approximated by $\omega(q)=m_{g}$ for $q \leq m_{g}$ and by $\omega(q)=q$ for $q>m_{g}$, with the constituent gluon mass given by $m_{g} \simeq 600 \mathrm{MeV}$ [192].

As usual, the gauge field is expressed as a superposition of plane wave modes with creation and annihilation operators $\alpha_{\lambda}^{a \dagger}(\boldsymbol{q})$ and $\alpha_{\lambda}^{a}(\boldsymbol{q})$, with $\lambda= \pm 1$ the polarization of the gluon. A hybrid $Q \bar{Q} g$ state is then obtained from the $\mathrm{BCS}$ vacuum as

$$
|r, \boldsymbol{q}, \lambda\rangle \propto Q_{\frac{r}{2} \boldsymbol{e}_{z}}^{\dagger} \alpha_{\lambda}^{a \dagger}(\boldsymbol{q}) T^{a} \bar{Q}_{-\frac{r}{2} \boldsymbol{e}_{z}}^{\dagger}|\Omega\rangle
$$

where $r$ is the separation between the heavy quark and the heavy antiquark (lying along the $z$-axis), and we introduced the corresponding creation operators. The energies $E_{n}$ are then obtained from Eq. (7.16).

This framework has been employed in [189] to compute the spectrum for the lowest lying hybrids as a function of the $Q \bar{Q}$ separation, and compare it with Lattice QCD data. It nicely fits the data for the ground state but it consistently overestimates them for excited gluonic fields. However, it correctly reproduces the ordering of the levels with quantum numbers. It was shown in [187] that this is a non-trivial result since many phenomenological models based on the flux tube description or on the Nambu-Goto action fail at this task. 
The analysis has been improved in [188]. In particular, it is argued that the solution of the $\partial\left\langle\psi_{0}\left|H_{\mathrm{QCD}}\right| \psi_{0}\right\rangle / \partial \omega(q)=0$ equation presents some ambiguities and it is therefore more sound to compute $\omega(q)$ phenomenologically from the spectrum of single gluon excitations, the so-called gluelumps [193]. Despite the improvement, the predictions are still systematically higher than the Lattice results. This might be due to intrinsic limitations of the gaussian, mean field, vacuum functional in Eq. (7.17). These techniques have also been used in [190] to study the radiative decays of hybrids to charmonia.

\subsubsection{The hybrid meson description of the $X Y Z$ states}

The hybrid meson model described so far has been mostly employed to explain the nature of the $Y(4260)$ [194196], but some proposal has also been made for the $Y(4360)$ [197], the $X(3872)$ [198] and the $X(4140)$ [199].

In [194] arguments in favor of a hybrid interpretation of the $Y(4260)$ are made. In particular, it would be constituted of a $J^{P C}=0^{-+} c \bar{c}$ pair together with a $J^{P C}=1^{+-}$gluonic excitation. Estimates obtained from the previously explained flux tube model [186] as well as lattice QCD simulations [200] give the mass of the lowest hybrid to be around $M \simeq 4.0-4.2 \mathrm{GeV}$, in agreement with that of the $Y(4260)$. The fact that the decay width $\Gamma\left(Y(4260) \rightarrow e^{+} e^{-}\right)$is a factor of 4 smaller than that for the $\psi(3770)$ is explained with a general suppression of the lepton-lepton coupling for hybrid mesons [201]. This is mostly due to the smallness of the $c \bar{c}$ wave function at $r=0$. Also, the decay of hybrids into pairs of $1 S$ ordinary mesons (e.g. $D \bar{D}, D_{s}^{+} D_{s}^{-}$, etc.) is expected to be largely suppressed [202, 203], hence explaining the missing observation of such channels. Lastly, it is noted that a mixing of the $c \bar{c} g$ component of the $Y(4260)$ with the ordinary $c \bar{c}$ would not be allowed. In the BO approximation, in fact, the $Q \bar{Q}$ state and the gluon one decouple and states with different gluon occupation numbers are orthogonal.

One issue with the $c \bar{c} g$ interpretation of the $Y(4260)$ is that the decay into charmonium + light hadrons was also expected to be fairly small. This is in contrast with the observation of the $J / \psi \pi \pi$ final state with large branching ratio.

\subsubsection{An alternative kind of hybrid?}

A possible solution to this problem has been proposed in [196]. The $Z_{c}(3900)$ is tentatively interpreted as a different kind of hybrid, where the excited gluon is replaced by a light quark pair in the $\mathbf{8}_{c}$ representation. We will refer to this kind of particle as a $B O$ tetraquarks. ${ }^{45}$ In this framework the large branching fraction for the decay of the $Y(4260)$ into $J / \psi \pi \pi$ would simply be explained by the $Y(4260) \rightarrow Z_{c} \pi$ process, followed by the copious $Z_{c} \rightarrow J / \psi \pi$ decay. The first reaction simply happens via a conversion of the gluon into a pion and an adjoint $[q \bar{q}]_{\mathbf{8}_{c}}$ pair.

It is important to note that the BO tetraquarks presented in [196] are nothing but an alternative model for the description of the compact tetraquarks. For small objects of typical size around $1 \mathrm{fm}$, the distinction between different color arrangements of the internal quarks is artificial, since they all mix with each other. The separation between the diquarkonium presented in Section 4 and the present BO tetraquarks is made just for the sake of model building.

In any case, assuming the previously explained structures for the $Y(4260)$ and $Z_{c}(3900)$, the BO approximation has been used in $[196,204]$ to compute the spectrum of hybrids and BO tetraquarks.

The first thing to do is to find a set of quantum numbers of the gluonic excitation which are conserved in presence of the static $Q \bar{Q}$ pair. The first one is the eigenvalue, $\Lambda$, of $\left|\boldsymbol{e}_{r} \cdot \boldsymbol{J}_{\ell}\right|$, where $\boldsymbol{e}_{r}$ is the unit vector pointing along the separation between the $Q$ and the $\bar{Q}$ and $\boldsymbol{J}_{\ell}$ is the angular momentum of the light degree of freedom, i.e. either $g$ or $[q \bar{q}]_{\mathbf{8}_{c}}$. We then consider the product of the parity and charge conjugation of the light excitation, $\eta=(C P)_{\text {light }}$, and,

\footnotetext{
${ }^{45}$ Note that the ordinary $c \bar{c} g$ hybrid cannot accommodate charged states.
} 

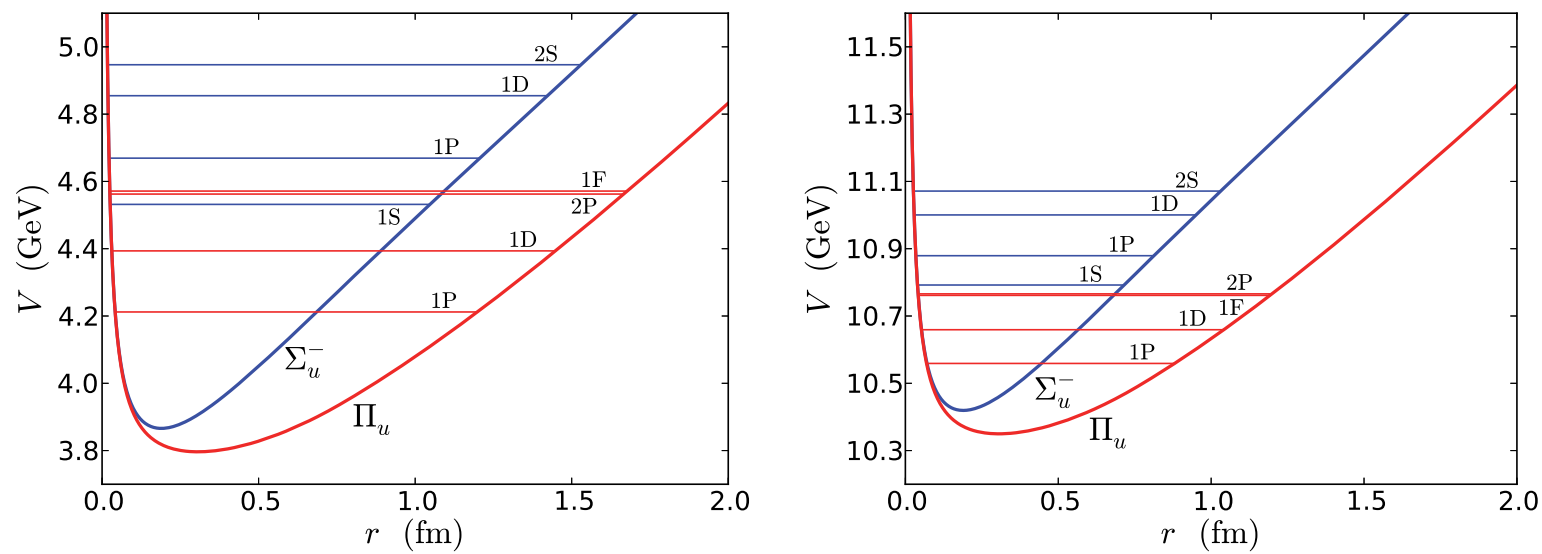

Figure 26: Lowest energy levels obtained from the solutions to Eq. (7.20) for the charm (left panel) and bottom (right panel) sectors. The notation is explained in the text. The analogous levels for the ground state of the gluon field, $\Sigma_{g}^{+}$, are just the ordinary quarkonia. Taken from [204].

when $\Lambda=0$, the eigenvalue, $\varepsilon= \pm$, of the reflection of the light fields with respect to a plane containing the $Q \bar{Q}$ system.

The notation adopted in [196] is that of molecular physics. In particular, the $\Lambda=0,1,2, \ldots$ states are represented as $\Sigma, \Pi, \Delta, \ldots$, and $\eta=+1$ and -1 are called respectively gerade $(g)$ and ungerade $(u)$. The quantum numbers of the light degrees of freedom are therefore indicated as $\alpha=\Sigma_{\eta}^{+}, \Sigma_{\eta}^{-}, \Pi_{\eta}, \Delta_{\eta}, \ldots$.

The strategy to compute the spectrum is the one already anticipated. In particular, the hybrid potential at some fixed separation $r$ of the $Q \bar{Q}$ pair is given by $V_{\alpha} \equiv V_{n_{\alpha}}$ as in Eq. (7.15) for large $r$, and by the following color-Coulomb potential for small $r$

$$
V_{\alpha}(r) \rightarrow \frac{\alpha_{s}(\mu)}{6 r}+E_{\alpha}
$$

Here the typical scale is $\mu=1 / r$ and $E_{\alpha}$ is an additive term depending on the quantum numbers $\alpha$ and usually called gluelump (see e.g. [205]). It can be thought as a massive quasi-particle excitation of the gauge field, a sort of constituent gluon. The different parameters of the potential can be obtained from Lattice QCD (see again Section 7.5).

Given the potential, the energy levels of the ordinary hybrid mesons are found by solving the following Schrödinger equations for the heavy pair

$$
\left[-\frac{1}{m_{Q}}\left(\frac{d}{d r}\right)^{2}+\frac{\left\langle\boldsymbol{L}_{Q \bar{Q}}\right\rangle_{\alpha, r}}{m_{Q} r^{2}}+V_{\alpha}(r)\right] \psi_{n}(r)=E_{n} \psi_{n}(r)
$$

where $\left\langle\boldsymbol{L}_{Q \bar{Q}}\right\rangle_{\alpha, r}$ is the angular momentum of the heavy quark pair computed at a certain separation $r$ and for a given set of quantum numbers $\alpha$ of the gluonic degrees of freedom, and $m_{Q}$ is the reduced mass of the $Q \bar{Q}$ system.

The $Q \bar{Q} g$ energy levels resulting from this calculation are reported in Figure 26. For the $c \bar{c} g$ case the lowest state is expected at $M \simeq 4246 \mathrm{MeV}$, while for the $b \bar{b} g$ one at $M \simeq 10559 \mathrm{MeV}$. While the first one is in agreement with the $Y(4260)$, no known resonance compatible with the hybrid interpretation has been found around the second mass.

To extract the spectrum of BO tetraquarks it is assumed in [196, 204] that their potential has the same qualitative behavior as that of $Q \bar{Q} g$ hybrids. This is a strong assumption since, except for the asymptotic behavior at small and 
larger $r$, very little is know about such potential. This makes the predictions substantially model-dependent. Moreover, it is also assumed that the BO tetraquarks potential for the $[q \bar{q}]_{\mathbf{8}_{c}}$ pair in the ground state, $\Sigma_{g}^{+}$, is completely unstable with respect to the transition into ordinary hybrid plus a light meson. It is therefore ignored. Again, this statement finds no rigorous ground but the practicality of the computation.

In Table 8 we reported the spectrum predicted in [196] for the BO tetraquarks in the charm sector. In particular, for each ordinary hybrid with quantum number $J^{P C}$ there will be a BO tetraquarks with the same $J^{P}$, with $I=1$ and hence $G=C(-1)^{I}=-C$. Because of HQSS - see Section $3.4-$ they will also organize themselves in quasi-degenerate spin multiples $T_{n}$. While the $Z_{c}$ is used as an input, the $Z_{c}^{\prime}$ would correspond to the $J^{P}=1^{+}$member of either $T_{3}$ or $T_{4}$.

\begin{tabular}{cc|cc|cc|cc}
\hline \hline \multicolumn{2}{c|}{$T_{1}$} & \multicolumn{2}{|c|}{$T_{2}$} & \multicolumn{2}{c|}{$T_{3}$} & \multicolumn{2}{c}{$T_{4}$} \\
\hline$I^{G}\left(J^{P}\right)$ & Mass $(\mathrm{MeV})$ & $I^{G}\left(J^{P}\right)$ & Mass $(\mathrm{MeV})$ & $I^{G}\left(J^{P}\right)$ & Mass $(\mathrm{MeV})$ & $I^{G}\left(J^{P}\right)$ & Mass $(\mathrm{MeV})$ \\
\hline $1^{+}\left(1^{-}\right)$ & 3839 & $1^{-}\left(1^{+}\right)$ & 3952 & $1^{-}\left(0^{+}\right)$ & 4025 & $1^{-}\left(2^{+}\right)$ & 4045 \\
$1^{-}\left(0^{-}\right)$ & 3748 & $1^{+}\left(0^{+}\right)$ & 3939 & $1^{+}\left(1^{+}\right)$ & 4030 & $1^{+}\left(1^{+}\right)$ & 4050 \\
$1^{-}\left(1^{-}\right)$ & 3770 & $1^{+}\left(1^{+}\right)$ & $\mathbf{3 8 9 7}$ & & & $1^{+}\left(2^{+}\right)$ & 4065 \\
$1^{-}\left(2^{-}\right)$ & 3887 & $1^{+}\left(2^{+}\right)$ & 3948 & & & $1^{+}\left(3^{+}\right)$ & 4101 \\
\hline \hline
\end{tabular}

Table 8: Predicted masses for the BO tetraquarks in the charm sector as reported in [196]. The bold mass corresponds to the $Z_{c}(3900)$ and is used as input.

As one can see, the BO tetraquarks model also suffers from the same drawbacks as for the diquarkonium. The zoology of predicted states is copious and largely unobserved.

\subsection{5. $X(3872)$ and $X(4140)$ are not hybrids}

Lastly, it is worth mentioning that the interpretation of the $X(3872)$ in terms of a $c \bar{c} g$ proposed soon after its discovery [198] has been ruled out by the confirmation of the $X \rightarrow J / \psi \rho$ decay. The hybrid model cannot account for isospin violation since $Q \bar{Q} g$ is always an $I=0$ state.

The interpretation of the $X(4140)$ as an hybrid [199] relies, instead, on its quantum numbers being $J^{P C}=1^{-+}$. This possibility has been ruled out by the recent $\mathrm{LHCb}$ analysis, which assign $1^{++}$to the state, see Section 8.6. Moreover, the decay of the gluon should be dominated by the creation of $u$ and $d$ quarks rather than $s$, as for the $X(4140) \rightarrow J / \psi \phi$ decay.
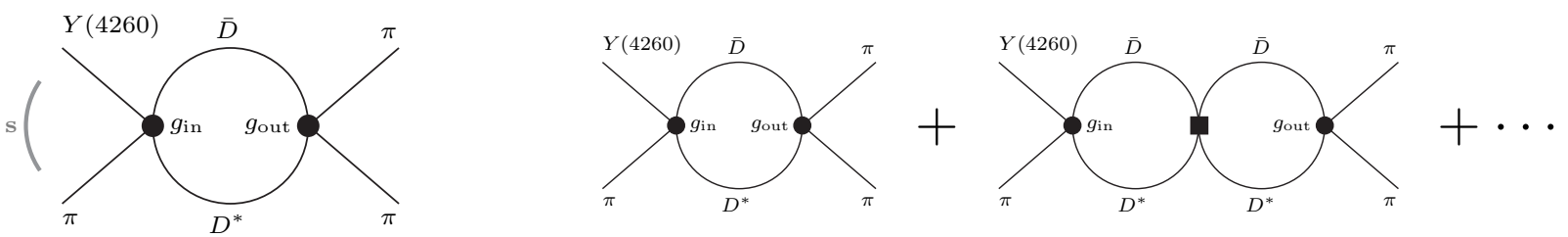

Figure 27: Left panel: One-loop diagram for the scattering $Y(4260) \pi \rightarrow J / \psi \pi$. Right panel: Unitarization of the one-loop diagram. 


\subsection{Nothing: kinematical effects}

Another option that has been widely discussed in the literature arises from the properties of the scattering amplitudes. Imposing unitarity and analyticity can indeed give rise to singularities in the complex plane, that are not related to resonances, but might mimic their behavior creating peaks and rapid motion of the phase shift. The idea and the formalism is known since long time [206]. Work has been done in the context of XYZ physics in [207-210]. To simplify the discussion, in the following we will neglect all the spins, and we will take the $D$ and $D^{*}$ to be degenerate in mass.

Consider, for example, the process $Y(4260) \rightarrow J / \psi \pi \pi$. We discuss the kinematic effects that can give origin to a peak in the $J / \psi \pi$ invariant mass compatible with the $Z_{c}$ (3900) (see Section 8.2) but without the presence of any real resonance. More precisely, consider the scattering process $Y \pi \rightarrow J / \psi \pi$, which is described by the same amplitude as the decay process (as a consequence of crossing symmetry). One can assume for the process to be dominated by the diagram in the left panel of Figure 27. The amplitude can thus be calculated with the usual Feynman rules, but the analytical structure emerges more transparently by using the Cutkosky rules, which give the imaginary part of the diagram, and the dispersion relations to calculate the real part. The former is

$$
\operatorname{Im} \Pi(s)=\frac{k_{\mathrm{i}}(s)}{4 \pi \sqrt{s}} g_{\text {in }}(s) g_{\text {out }}(s) \theta\left(s-4 m_{D}^{2}\right)
$$

where $k_{\mathrm{i}}=\frac{1}{2} \sqrt{s-4 m_{D}^{2}}$ is the 3 -momentum of the $\bar{D}$ and $D^{*}$ in the center-of-mass frame, and $g_{\text {in,out }}$ are form factors. The dispersive integral relating the full amplitude to its imaginary part is

$$
\Pi(s)=\frac{1}{\pi} \int_{4 m_{D}^{2}}^{\infty} d s^{\prime} \frac{\operatorname{Im} \Pi\left(s^{\prime}\right)}{s^{\prime}-s-i \epsilon}
$$

The integral can be solved, even explicitly for the case of constant $g$ 's, in which case the solution is given by $\sqrt{s-4 m_{D}^{2}}$ times a logarithm. Because of the square root, this function has a cusp exactly at threshold, as shown in the left panel of Figure 28. Form factors suggested by the quark model, like $g \propto \exp \left(-s / \beta^{2}\right)$ have often been used, even though they are not consistent with the analytical behavior of the amplitude (which cannot grow faster than a polynomial at infinity in the complex plane).

However, this simple model does not take into account the unitarization. The simplest way to achieve a unitary amplitude is to resum the 1-loop diagrams (Figure 27, right panel), the one-loop expression goes to the denominator of the amplitude, $A(s)=\sum_{n}[\Pi(s)]^{n}=1 /(1-\Pi(s))$, which develops a pole when $\Pi(s)=1$, i.e. a proper QCD state. The right panel of Figure 28 shows that, if one still wants to stick to the one-loop diagram only, the restriction to a coupling small enough to give meaning to the perturbative expansion does not allow to fit data correctly [211]. If the coupling is instead nonpertubative, each order of the expansion gives a drastically different result (dotted and dashed curves in Figure 28) and one cannot avoid unitarization.

In the previous discussion, the $Y \pi \rightarrow \bar{D} D^{*}$ vertex was parametrized with a smooth function $g_{\text {in }}(s)$. However, this is just an approximation for an actual exchange of particles, if their mass are heavy enough to contract their propagator. One can investigate instead if an appropriate model for the particle exchange produces any singularity close to the physical region. Triangle diagrams develop a logarithmic branch point ${ }^{46}$. Consider again the $Y \rightarrow J / \psi \pi \pi$ process, but now described by the diagram in the left panel of Figure 29. We consider the mesons in the loop to be the $D$, the $D^{*}$, and a generic charmed meson of mass $\lambda$. We want to study the behavior of the amplitude as a function of the mass $\lambda$,

\footnotetext{
${ }^{46}$ Landau classified the leading singularities that a $n$-point amplitude can have in perturbation theory. The diagrams in Figure 27 are basically two-points function (the four momenta enter only through the $s$ Mandelstam variable), and they exhibit a square root singularities. The triangle diagrams exhibit a logarithmic singularities, and so on.
} 

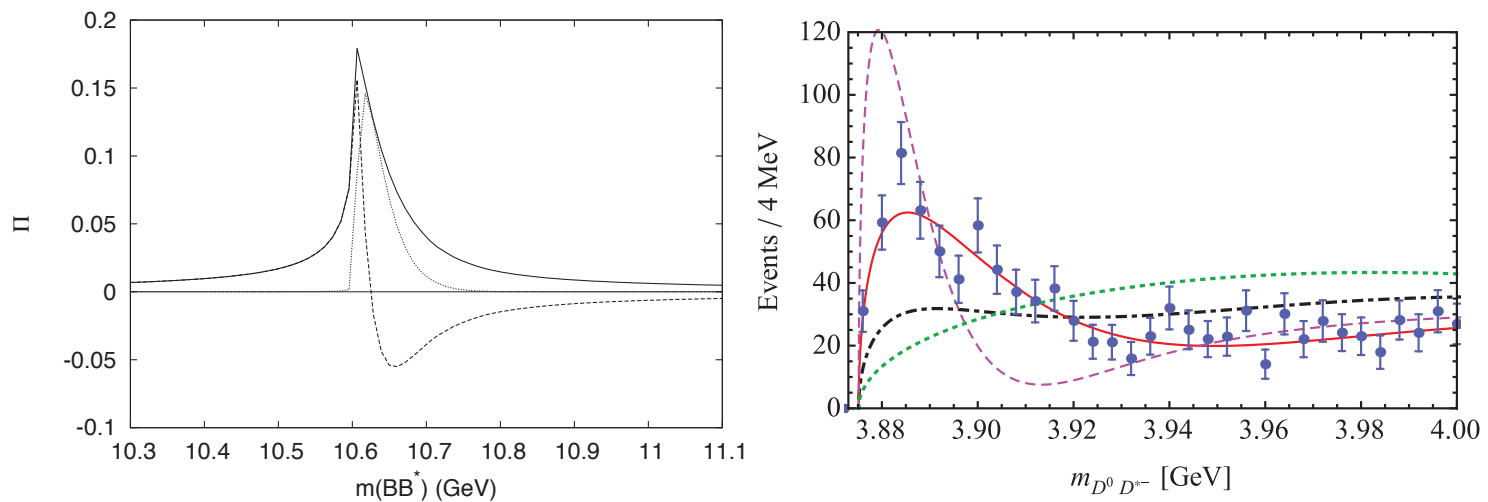

Figure 28: Left panel: Plot of the $\Pi$ amplitude, showing the square root behavior of the imaginary part (dotted), the real part obtained via dispersion relation (dashed), and the modulus (solid), from [210]. Right Panel: The invariant $\bar{D} D^{*}$ mass distribution in $Y(4260) \rightarrow \pi \bar{D} D^{*}$ fitted in [211]. The results from the tree level, one-loop and two-loop calculations are shown by the dotted, solid and dashed curves, respectively. The dot-dashed line shows the one-loop result with the strength of the rescattering requested to be small to justify a perturbative treatment.
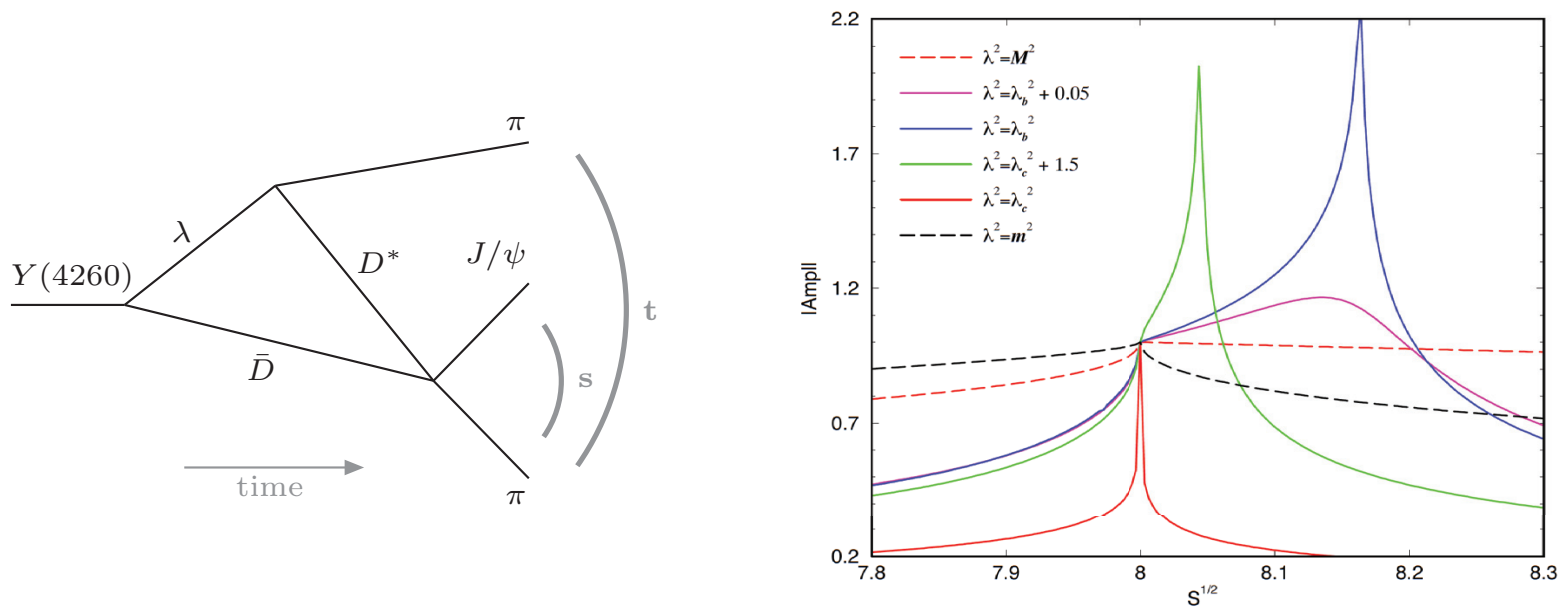

Figure 29: Left panel: Triangle diagram occurring in $Y(4260) \rightarrow J / \psi \pi \pi$ process. Right panel: values of the amplitude for different values of $\lambda$. If an actual state happen to fall in the small window which fulfills the Coleman-Norton conditions, a cusp appear. From [212].

and see if any peak in the $J / \psi \pi$ invariant mass can occur. Again, we focus on the crossed process $Y \pi \rightarrow J / \psi \pi$. Since the centrifugal barriers will suppress any amplitude close to threshold, we consider only the $S$-wave projection. The $\lambda$ meson is exchanged in the $t$ channel, so the projection of the propagator gives

$$
C(s)=\frac{1}{2} \int_{-1}^{1} d \cos \theta \frac{C}{t(s, \cos \theta)-\lambda^{2}+i \epsilon} P_{0}(\cos \theta)
$$

where $C$ is a coupling constant, $P_{0}(\cos \theta)=1$ is the $L=0$ Legendre polynomial. In the center of mass frame, $t=2 m_{\pi}^{2}-2 E_{\pi}^{\text {in }} E_{\pi}^{\text {out }}+2 p^{\text {in }} p^{\text {out }} \cos \theta$, which is linear in $\cos \theta$. The integral thus gives a logarithm,

$$
C(s)=-\frac{C}{2 p^{\text {in }} p^{\text {out }}} Q_{0}\left(\frac{E_{\pi}^{\text {in }} E_{\pi}^{\text {out }}-m_{\pi}^{2}-\frac{1}{2} \lambda^{2}-i \epsilon}{p^{\text {in }} p^{\text {fin }}}\right)
$$

where $Q_{0}(x)=\frac{1}{2} \log \frac{x+1}{x-1}$ is the Legendre function of the second kind. The unitarity of the $S$-matrix can be rewritten as an equation for the discontinuity of the analytic continuation of the amplitude across the physical axis

$$
\operatorname{disc} A(s)=\frac{1}{2 i}[A(s+i \epsilon)-A(s-i \epsilon)]=C(s) \rho(s) B^{*}(s) \theta\left(s-4 m_{D}^{2}\right)
$$


where $\rho(s)=\sqrt{1-4 m_{D}^{2} / s}$ is the 2-body phase space, and $B$ the scattering amplitude $\bar{D} D^{*} \rightarrow J / \psi \pi$, which we approximate as a constant. The dispersion relation on $A$ reads

$$
A(s)=\frac{1}{\pi} \int_{4 M_{D}^{2}}^{\infty} d s^{\prime} \frac{C\left(s^{\prime}\right) \rho\left(s^{\prime}\right) B}{s^{\prime}-s}
$$

The analytical structure of $C\left(s^{\prime}\right)$ is rather complicated, and can contain up to four different branch points. In general they do not produce sizable effect in $A(s)$ : even though one of the $s^{\prime}$ branch points is close to the real axis, one can deform the integration contour to distance it. However, Coleman and Norton show that when all the particles in the loop are simultaneously on-shell, and their velocities are such that the the rescattering can occur in real time, two branch points in $s^{\prime}$ pinch the $s$ contour of integration, preventing the deformation of the contour. This produces cusps in $A(s)$ close to the real axis [213]. This happens for a very small window of $\lambda$, see for example the effects in the right panel of Figure 29. In particular, the $D_{1}(2420)$ mass happens to be in this interval, and might produce an enhancement in correspondence of the $Z_{c}$.

Now, let us consider the $Y \rightarrow \bar{D} D^{*} \pi$ process. If no actual $Z_{c}(3900)$ exists, the Dalitz plot will exhibit a band in the $D^{*} \pi$ channel corresponding to the $D_{1}(2420)$, and a band in the $\bar{D} D^{*}$ channel due to the triangle singularity. Longtime ago, Schmid proved that the interference between the two bands cancels the contribution of the $\bar{D} D^{*}$ band: the projection on the $\bar{D} D^{*}$ invariant mass does not produce any peaks [214]. The situation is more complicated when multiple channel are involved. In this case, the compensation happens among the various channel, and if one focuses only on a given channel, the peak can happen to be still be evident [215]. If this is the mechanism behind the peak, the complete coupled channel analysis will show a rather peculiar behavior: one expects a depletion of events in the channel where the triangle singularity occurs, and an enhancement in the other channels. Present data in the $Y \rightarrow \bar{D} D^{*} \pi$ channel do not seem to favor this hypothesis, but his can be verified in future high statistics experiments. The role of triangle singularities for $X Y Z P$ states has been discussed in [212, 215-220].

\subsection{QCD sum rules}

A technique often used to compute the mass, width and coupling constants of the exotic states is the QCD Sum Rules (QCDSR) - see [221] for a review. The method was first appeared in [222], and was later developed in [223, 224]. It was used to study the properties of mesons. The focus is on the evaluation of a two-point correlation function as

$$
\Pi(q) \equiv i \int d^{4} x e^{i q x}\left\langle 0\left|T\left(j(x) j^{\dagger}(0)\right)\right| 0\right\rangle
$$

where $j(x)$ is a current with the quantum numbers of the hadron we want to study. The choice of the current $j(x)$ is only dictated by the $\left(I^{G}\right) J^{P C}$ quantum numbers of the hadron and by assumptions on its nature. In general, it can be a linear superposition of currents corresponding to different structures (quarkonium, diquarkonium, meson molecule, etc.).

The important assumption is that this correlator can be evaluated both from the fundamental degrees of freedom of QCD (the so-called OPE side) and from an effective meson theory (the so-called phenomenological side). This means that, one starts from the asymptotically free theory of quarks and gluons towards non-perturbative scales. The assumption is that, in a certain range of scales, the two theories will give the same result for the correlator (7.27).

On the OPE side, one expands the function as a series of local operators

$$
\Pi_{\mathrm{OPE}}\left(q^{2}\right)=\sum_{n} C_{n}\left(Q^{2}\right) O_{n}
$$


with $Q^{2}=-q^{2}$ and where the set $\left\{O_{n}\right\}$ includes all the local, gauge-invariant operators that can be written in terms of the gluon and quark fields. They are also ordered by mass dimension. The information about the short-range, perturbative part of the correlator is (by construction) contained in the Wilson coefficients $C_{n}\left(Q^{2}\right)$. The matrix elements for the operators $O_{n}$ are nonpertubative and must be evaluated through Lattice QCD or using some phenomenological model. They are universal, i.e. once they are fixed using a certain observable like the mass of $a$ particle they cannot be changed. The same thing is true for the quark masses.

On the phenomenological side, instead, one writes the two-point function in terms of a spectral density $\rho(s)$

$$
\Pi_{\text {phen }}\left(q^{2}\right)=-\int d s \frac{\rho(s)}{\left(q^{2}-s+i \epsilon\right)}+\cdots
$$

with the dots representing subtraction terms. In order for the previous equation to be of practical use, the spectral density has to be parametrized in terms of a small number of parameters. One usually assumes that it has a pole corresponding to the mass of the ground-state hadron, while higher mass states are contained in a smooth, continuous part

$$
\rho(s)=\lambda^{2} \delta\left(s-m^{2}\right)+\rho_{\text {cont }}(s)
$$

$\lambda$ being the coupling of the current to the lowest mass hadron, $H,\langle 0|j| H\rangle=\lambda$. Moreover, the continuum contribution is taken to be zero below a certain threshold $s_{0}$, and to it coincide with the result obtained from the OPE side above it, i.e. one makes the ansatz

$$
\rho_{\text {cont }}(s)=\rho_{\mathrm{OPE}}(s) \theta\left(s-s_{0}\right)
$$

where $\pi \rho_{\mathrm{OPE}}(s)=\operatorname{Im} \Pi_{\mathrm{OPE}}(s)$. The threshold parameter $s_{0}$ is again universal. Combining Eqs. (7.29), (7.30) and (7.31) allows to extract the mass of the resonance.

The decay widths, i.e. the coupling constants, can be estimated with similar procedures but starting from the three-point correlator.

The previous formalism has been applied to study the properties of the $X(3872)$ [225], of the $Z_{c}^{(\prime)}$ [226-228], of the $Z_{b}^{(\prime)}[229,230]$ and of the $Y$ states [231]. The literature on the topic is impressively vast. We will report here just a limited number of results. The interested reader should refer to other reviews [221].

It should be stressed that QCD sum rules are supposed to be the result of first principle, field theoretic QCD, and as such they should provide reliable, univocal nonpertubative results. Unfortunately, there is some freedom in choosing the rules to compute the different observables. Moreover, some quantities can often be reproduced by suitably tuning the different compositions of the state under consideration. In this respects, the technique is often able to reproduce existing data, but its predictive power is somehow limited. In general, QCD sum rules are applicable to the lowest states in a channel with fixed quantum numbers. This is not the case for the exotic states discussed in this work. The truncated OPE expansion should lack of the theoretical precision necessary to extract informations relative to excited states. The following results should therefore always be taken with a grain of salt, as it will soon be clear from some of the following conclusions.

In [225] the mass of the $X(3872)$ and its $\Gamma\left(X \rightarrow J / \psi \pi^{+} \pi^{-}\right)$decay width are analyzed. They assumed the state to be a mixture of ordinary charmonium and meson molecule. The current in Eq. (7.27) is taken to be

$$
J_{\mu}^{u}(x)=\sin \theta j_{\mu}^{(4 u)}(x)+\cos \theta j_{\mu}^{(2 u)}(x)
$$

where $\theta$ is a mixing angle and $j_{\mu}^{(2 u)}$ and $j_{\mu}^{(4 u)}$ are current with the quantum numbers of charmonium and meson 
molecules respectively. They are given by

$$
\begin{aligned}
j_{\mu}^{(2 u)}(x) & =\frac{1}{6 \sqrt{2}}\langle\bar{u} u\rangle \bar{c}_{\alpha}(x) \gamma_{\mu} \gamma_{5} c^{\alpha}(x) \\
j^{(4 u)}=\mu(x) & =\frac{1}{\sqrt{2}}\left[\bar{u}_{\alpha}(x) \gamma_{5} c^{\alpha}(x) \bar{c}_{\beta}(x) \gamma_{\mu} u^{\beta}(x)-\bar{u}_{\alpha}(x) \gamma_{\mu} c^{\alpha}(x) \bar{c}_{\beta}(x) \gamma_{5} u^{\beta}(x)\right]
\end{aligned}
$$

Using the previous currents and the techniques explained above they estimated the mass and decay width of the $X$ to be $M_{X}=(3.77 \pm 0.18) \mathrm{GeV}$ and $\Gamma\left(X \rightarrow J / \psi \pi^{+} \pi^{-}\right)=(9.3 \pm 6.9) \mathrm{MeV}$, with a small mixing angle, $\theta \in\left[5^{\circ}, 13^{\circ}\right]$. They also managed to estimate the weights of the different components of the $X$ wave function. They found that it is composed for the $\sim 97 \%$ by ordinary charmonium and for the remaining $\sim 3 \%$ by a molecule. Of the latter one $\sim 88 \%$ is $D^{0} \bar{D}^{0 *}$ and $\sim 12 \%$ is $D^{+} D^{*-}$. Of course, today we know that these results are not compatible with experimental data. The $X(3872)$ is not a charmonium. The same authors studied the $X$ under the assumption of a diquarkonium internal structure [232] and found a mass in agreement with the observation.

In [226] the decays of $Z_{c}(3900)$ where studied assuming a diquarkonium structure. The authors found a total width $\Gamma=(63.0 \pm 18.1) \mathrm{MeV}$, and the following partial widths: $\Gamma\left(Z_{c} \rightarrow J / \psi \pi\right)=(29.1 \pm 8.2) \mathrm{MeV}, \Gamma\left(Z_{c} \rightarrow \eta_{c} \rho\right)=$ $(27.5 \pm 8.5 \mathrm{MeV})$ and $\Gamma\left(Z_{c} \rightarrow D D^{*}\right)=(3.2 \pm 0.7 \mathrm{MeV}$. The width is indeed compatible with the experimental one within $2 \sigma$, but the ration of the decay rates into $D D^{*}$ and into $J / \psi \pi$ is much smaller than the one reported in Eq. (8.12). A similar analysis was performed in [228] with analogous results.

The $Z_{b}$ is instead studied in [229] using a variety of molecular interpolating currents. The extracted masses are nicely compatible with experiment. On the other hand, the properties of the $Z_{b}$ and $Z_{b}^{\prime}$ are analyzed in [230] assuming a diquarkonium nature. The masses are very much in agreement with the observation and the predictions for the decay widths of the $Z_{b}$ give $\Gamma\left(Z_{b} \rightarrow \Upsilon \pi\right)=\left(4.77_{-2.46}^{+3.27}\right) \mathrm{MeV}$ and $\Gamma\left(Z_{b} \rightarrow \eta_{c} \rho^{+}\right)=\left(13.52_{-6.93}^{+8.89}\right) \mathrm{MeV}$. While the first one agrees with the the experiment, no data on the second decay channel are available yet.

Lastly, the application of QCD sum rules to the $Y$ states has been throughly done in [231]. The authors find support for the diquarkonium nature of the $Y(4660)$ and for the mixed charmonium-diquarkonium for the $Y(4260)$ and $Y(4360)$.

\subsection{Lattice $Q C D$}

Some efforts in trying to confirm the existence and understand the nature of the exotic states have been made using Lattice QCD techiniques — see $[233,234]$ for an extensive treatment. Let us briefly recall the underlying strategy.

For a given set of quantum numbers one usually considers an ensemble of interpolating operators, $O_{i}(t)$, compatible with those quantum numbers. Once they are identified, it is possible to study their two-point correlators, which can be written as

$$
\left\langle 0\left|O_{i}^{\dagger}(t) O_{j}(0)\right| 0\right\rangle=\bigvee_{n}\left\langle 0\left|e^{i H t} O_{i}^{\dagger}(0) e^{-i H t}\right| n\right\rangle\left\langle n\left|O_{j}(0)\right| 0\right\rangle=\bigvee_{n} Z_{i}^{n *} Z_{j}^{n} e^{-i E_{n} t}
$$

where we defined the overlaps $Z_{i}^{n} \equiv\left\langle n\left|O_{i}(0)\right| 0\right\rangle$. The $|n\rangle$ are the multiparticle eigenstates with given quantum numbers. We remark that in the infinite volume their spectrum is obviously continuous.

To make the previous oscillating sum suitable to numerical studies, one performs an analytic continuation to Euclidean time $(t \rightarrow-i \tau)$. Spacetime is then discretized, with a lattice spacing $a$, and the analysis is restricted to a finite box with size $L$, in which case the correlator becomes

$$
C_{i j}\left(N_{\tau}\right)=\left\langle O_{i}^{\dagger}(\tau) O_{j}(0)\right\rangle=\sum_{n=1}^{\infty} Z_{i}^{n *} Z_{j}^{n} e^{-E_{n} a \tau},
$$


with $\tau$ integer, $0 \leq \tau \leq L / a$. Since the allowed momenta are now discretized, the physical states are now a discrete set. To extract the mass of the lowest lying level, the so-called effective mass method can be used. One defines

$$
M_{(i j)}^{\mathrm{eff}}(\tau)=\log \frac{C_{i j}(\tau-1)}{C_{i j}(\tau)}
$$

For large $\tau$, the previous expression converges to the mass of the lowest energy state. For a realistic simulation with a finite range in the Euclidean time $\tau$, it will exhibit a plateau, from which the spectrum can be fitted. To improve the reliability of the results one usually combines data obtained from different interpolating operators.

Other ways to determine the presence of a resonance and the value of its mass are to solve the generalized eigenvalue problem (GEP) [235], or to employ the so-called Lüscher's method [236, 237], which uses the dependence of the correlators from the size of the box to extract information about the scattering phase shift in the infinite volume limit.

It should be stressed that the impact that Lattice QCD analyses can have on the study of the $X Y Z$ states is limited by some technical issues, which can hardly be overcome with the present resources. Some of these problems are

- Any operator $O_{i}$ able to resolve an exotic state is also able to resolve other states with the same quantum numbers. For example, if $O_{i}$ interpolates the charged $Z_{c}(3900)^{+}$with $J^{P C}=1^{+-}$then it will also give access to the $b_{1}^{+}$and all its excitations. In principle, one should extract the full spectrum of the $b_{1}$ up to the mass of the $Z_{c}$, which is not feasible. In practice, one neglects the charm quark annihilation diagrams, which are expected to give a small contribution because of the OZI rule. This also prevents the mixing with hadrons made of light quarks only (like the $b_{1}$ ). However, the relevance of these diagrams for such fine-tuned systems like the $X Y Z$ states is presently unknown.

- It should be clear by now that the problem at hand is made even more non-trivial by the presence of several close by meson-meson threshold. To be able to distinguish between one of the $X Y Z$ resonances and a simple threshold requires a resolution that is right now far from achievable. For example, to have a precision of, say, $\Delta E \sim 10 \mathrm{MeV}$ one would need a lattice spacing of at least $a \sim 1 / \Delta E \sim 20 \mathrm{fm}$. Realistic lattices have typically $a$ of the order of a few tens of a fm, with a total box size of a few fm itself.

- If these exotic resonances are indeed molecular states, as proposed in part of the literature, the smallness of their binding energy would also imply a large size in real space — see Sections 3.2 and 3.3. In this case, the total size of the box would be even smaller than the total extension of the state.

Despite the previous limitations, some attempts to look for four-quark resonances have been made.

The simplest lattice calculations involve infinitely heavy quarks (static limit). Although in this limit it is not possible to have reliable predictions on the spectrum and decay properties of the states, it is a clean environment to study the interquark potential (which in this limit is a well defined quantity), and to provide a ground basis for the different models and phenomenology. We already cited the work by Lucini et al. [115], which observed some evidence for the formation of a scalar diquark, and the work by Cardoso et al. [77], where there is evidence that the $q q \bar{q} \bar{q}$ system indeed arranges itself in the expected $\mathrm{H}$ shaped configuration. A new method to study this setup has been proposed in [238], and might give new information about the diquark formations. Also system with two static quarks and two light dynamical antiquarks have been explored [239-242].

The only positive result for the existence of tetraquark resonances has been obtained in [243] for the $X(3872)$. The authors performed an analysis with valence and dynamical $u$ and $d$ quarks, with $m_{u}=m_{d}$ and a pion mass $m_{\pi}=266 \mathrm{MeV}$. Charm annihilation diagrams were also neglected. The authors did not consider the mixing of different partial waves due to the nonzero spin, nor the proper identification of the spin in terms of representations of 
the cubic group. They studied both the isospin $I=0$ and $I=1$ cases. Among the obtained levels they observe one very close to the $D D^{*}$ threshold, and which they interpret as the $X(3872)$. It should be stressed that, for the reasons explained above, there is also the possibility for such level to correspond to the ordinary $\chi_{c 1}(2 P)$ charmonium, which carries that same quantum numbers as the $X$.

All the other searches for $X Y Z$ states on the lattice (including manifestly exotic states with non-zero flavor charge [244-246]) have returned inconclusive results.

Some analyses have also been done to study hybrid mesons. In this case, the results are more reliable since one can employ the Born-Oppenheimer approximation to describe a system with a heavy $Q \bar{Q}$ pair and a light degree of freedom (see Section 7.2). In particular, although the phenomenological application of these states to the study of $X Y Z$ resonances is problematic, their existence in the QCD spectrum has now been well assessed. In the bottom sector, the hybrid potential and the corresponding energy levels have been found in [247, 248]. The state of the art for the $c \bar{c}$ spectroscopy, including hybrid states, has instead been set by [249], albeit in the one-particle (zero width) approximation.

\section{A travel guide to experimental results}

We present here a short summary of the most important experimental results in the exotic XYZP sector. This chapter does not aim to cover entirely the large number of papers published on this topic, but rather to give a synthetic review of the aspects which appear more relevant from the theoretical point of view. In doing so, we will not follow the chronological order. Whenever available, we will calculate averages using the new published measurement, to update the results shown in the PDG 2014. For the $X(3872)$, we show the result of a global fit to all available data, to provide an estimate of the absolute branching fractions.

The landscape of exotic resonances is summarized in Figure 30, and in Tables 9 and 10. Some states, like the $X(3872)$ or the $X(3915)$, have more or less the correct mass and quantum numbers of some missing ordinary charmonia; on the other hand, in the vector sector we have much more levels than expected. In any case, the decay pattern of all these states is not compatible with charmonia predictions, and so it calls for exotic interpretations. No ordinary assignment is possible for the charged charmoniumlike and bottomoniumlike states, nor for the new hidden-charm pentaquarks.

Depending on the available energies and colliding particles, the various experiments can exploit different production modes of the exotic states. Some of these can constrain the quantum numbers assignments. A generic state $X$ can be produced

- Directly with $e^{+} e^{-} \rightarrow X$, or in association with Initial State Radiation (ISR) which lowers the center of mass energy, $e^{+} e^{-} \rightarrow e^{+} e^{-} \gamma_{\mathrm{ISR}} \rightarrow X \gamma_{\mathrm{ISR}}$. The quantum numbers must be the same as the photon, $J^{P C}=1^{--}$. The $Y$ states discovered with this technique are discussed in Section 8.4. The $\tau-c$ and the $B$-factories can directly explore the charmonium and bottomonium vectors, respectively. This is what BES III did, collecting a large data sample at the $Y(4260)$ peak. The $B$-factories (BABAR and Belle) can also perform ISR analyses on the charmonium sector.

- In the fusion of two quasi-real photons, $e^{+} e^{-} \rightarrow e^{+} e^{-} \gamma \gamma \rightarrow e^{+} e^{-} X$, where $e^{+}$and $e^{-}$are scattered at a small angle and are lost in the beampipe; the signal events have no tracks and neutral particles but the daughters of $X$. If the photons are quasi-real, Landau-Yang theorem holds [287], and $J \neq 1$; moreover $C=+$ is constrained. The $B$-factories discovered the $\chi_{c 0}(3915)$ and the $X(4350)$ with this technique (Section 8.8). 

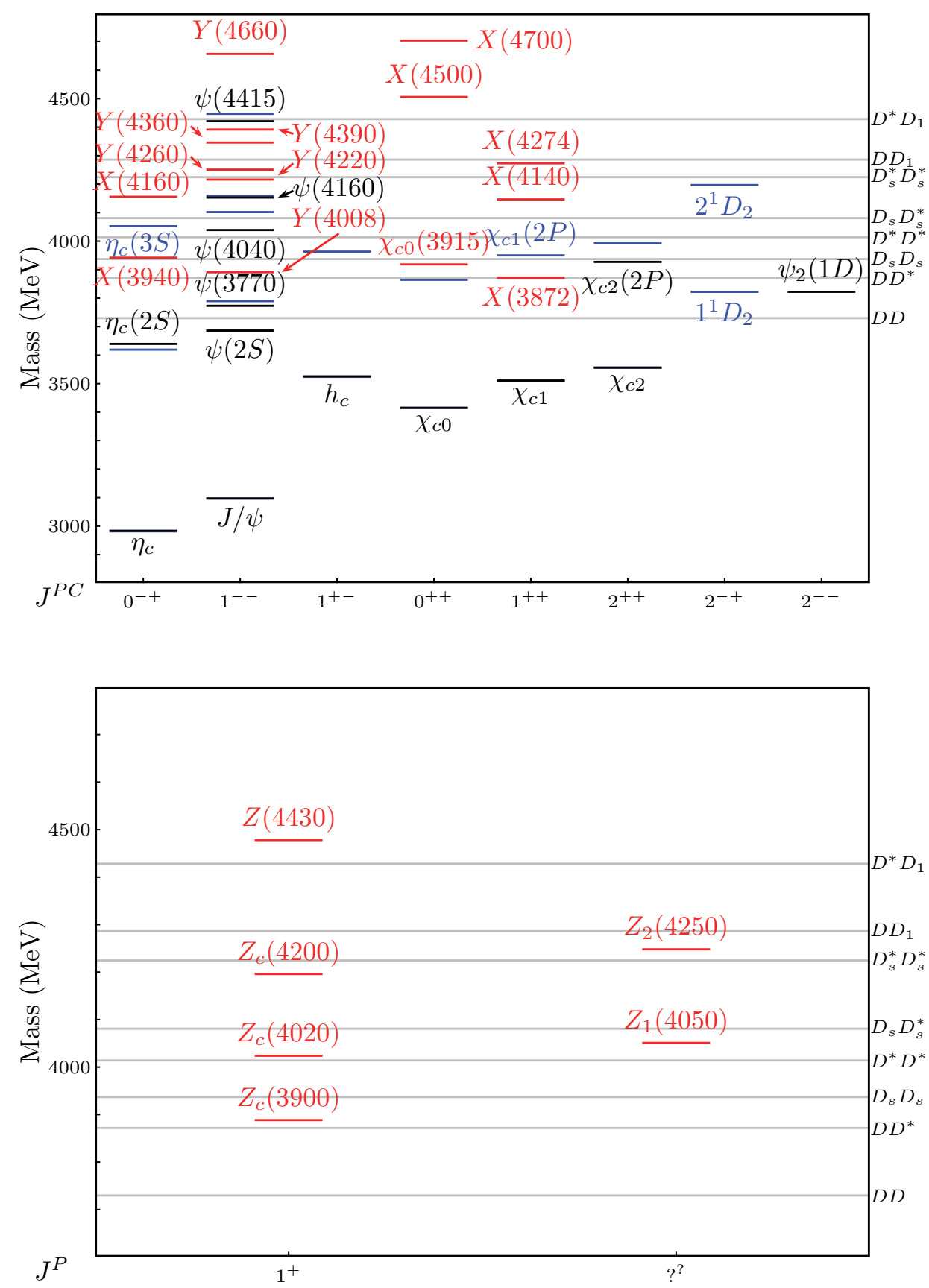

Figure 30: Charmonium sector. In the upper panel, we show ordinary charmonia and neutral exotic states, in the lower panel charged exotic states. Black lines represent observed charmonium levels, blue lines represent predicted levels according to Radford and Repko [250], red line are exotic states. The open charm thresholds are reported on the right. 


\begin{tabular}{|c|c|c|c|c|c|}
\hline State & $M(\mathrm{MeV})$ & $\Gamma(\mathrm{MeV})$ & $J^{P C}$ & Process (mode) & Experiment $(\# \sigma)$ \\
\hline$X(3872)$ & $3871.69 \pm 0.17$ & $<1.2$ & $1^{++}$ & See Table 11,12 & See Table 11,12 \\
\hline \multirow[t]{3}{*}{$Z_{c}(3900)^{+}$} & $3888.4 \pm 1.6$ & $27.9 \pm 2.7$ & $1^{+-}$ & $Y(4260) \rightarrow \pi^{-}\left(D \bar{D}^{*}\right)^{+}$ & BES III $[159,251](>10)$ \\
\hline & & & & $Y(4260) \rightarrow \pi^{-}\left(\pi^{+} J / \psi\right)$ & BES III [156] (8), Belle [155] (5.2) \\
\hline & & & & & NU group [252] (3.5) \\
\hline \multirow[t]{3}{*}{$Z_{c}(3900)^{0}$} & $3893.6 \pm 3.7$ & $31 \pm 10$ & $1^{+-}$ & $Y(4260) \rightarrow \pi^{0}\left(D \bar{D}^{*}\right)^{0}$ & BES III [253] (10) \\
\hline & & & & $Y(4260) \rightarrow \pi^{0}\left(\pi^{0} J / \psi\right)$ & BES III [156] (10.4) \\
\hline & & & & & NU group [252] (5.7) \\
\hline \multirow[t]{2}{*}{$Z_{c}^{\prime}(4020)^{+}$} & $4023.9 \pm 2.4$ & $10 \pm 6$ & $1^{+-}$ & $e^{+} e^{-} \rightarrow \pi^{-}\left(\pi^{+} h_{c}\right)$ & BES III [161] (8.9) \\
\hline & & & & $e^{+} e^{-} \rightarrow \pi^{-}\left(D^{*} \bar{D}^{*}\right)^{+}$ & BES III [254] (10) \\
\hline \multirow[t]{2}{*}{$Z_{c}^{\prime}(4020)^{0}$} & $4024.5 \pm 3.1$ & $23 \pm 6 \pm 1$ & $1^{+-}$ & $e^{+} e^{-} \rightarrow \pi^{0}\left(\pi^{0} h_{c}\right)$ & BES III [255] (5) \\
\hline & & & & $e^{+} e^{-} \rightarrow \pi^{0}\left(D^{*} \bar{D}^{*}\right)^{0}$ & BES III [256] (5.9) \\
\hline \multirow[t]{2}{*}{$\chi_{c 0}(3915)$} & $3918.4 \pm 1.9$ & $20 \pm 5$ & $0^{++}$ & $B \rightarrow K(\omega J / \psi)$ & Belle [257] (8), BABAR [258, 259] (19) \\
\hline & & & & $e^{+} e^{-} \rightarrow e^{+} e^{-}(\omega \mathrm{J} / \psi)$ & Belle [260] (7.7), BABAR [261] (7.6) \\
\hline$X(3940)$ & $3942_{-8}^{+9}$ & $37_{-17}^{+27}$ & $\left(0^{-+}\right)$ & $e^{+} e^{-} \rightarrow J / \psi\left(D \bar{D}^{*}\right)$ & Belle $[262,263](6)$ \\
\hline \multirow{2}{*}{$(Y(4008) ?)$} & $3891 \pm 42$ & $255 \pm 42$ & \multirow{2}{*}{$1^{--}$} & \multirow{2}{*}{$e^{+} e^{-} \rightarrow\left(\pi^{+} \pi^{-} J / \psi\right)$} & Belle $[155,264](7.4)$ \\
\hline & $3813_{-97}^{+62}$ & $477_{-65}^{+78}$ & & & BES III [108] (np) \\
\hline$Z(4050)^{+}$ & $4051_{-43}^{+24}$ & $82_{-55}^{+51}$ & $?^{?+}$ & $\bar{B}^{0} \rightarrow K^{-}\left(\pi^{+} \chi_{c 1}\right)$ & Belle [265] (5.0), BABAR [266] (1.1) \\
\hline$\left(Z(4050)^{+} ?\right)$ & $4054 \pm 3$ & $45 \pm 13$ & $?^{?-}$ & $e^{+} e^{-} \rightarrow\left(\psi(2 S) \pi^{+}\right) \pi^{-}$ & Belle [267] (3.5) \\
\hline$X(4140)$ & $4146.5_{-5.3}^{+6.4}$ & $83_{-25}^{+30}$ & $1^{++}$ & $B^{+} \rightarrow(J / \psi \phi) K^{+}$ & LHCb $[135,136](8.4)$, see Table 16 \\
\hline$X(4160)$ & $4156_{-25}^{+29}$ & $139_{-65}^{+113}$ & $\left(0^{-+}\right)$ & $e^{+} e^{-} \rightarrow J / \psi\left(D^{*} \bar{D}^{*}\right)$ & Belle [263] (5.5) \\
\hline$Z(4200)^{+}$ & $4196_{-30}^{+35}$ & $370_{-110}^{+99}$ & $1^{+-}$ & $\bar{B}^{0} \rightarrow K^{-}\left(\pi^{+} J / \psi\right)$ & Belle [268] (7.2) \\
\hline$Y(4220)$ & $4218.4 \pm 4.1$ & $66.0 \pm 9.0$ & $1^{--}$ & $e^{+} e^{-} \rightarrow\left(\pi^{+} \pi^{-} h_{c}\right)$ & BES III [108] (np) \\
\hline$Y(4230)$ & $4230 \pm 8$ & $38 \pm 12$ & $1^{--}$ & $e^{+} e^{-} \rightarrow\left(\chi_{c 0} \omega\right)$ & BES III [269] (> 9) \\
\hline$Z(4250)^{+}$ & $4248_{-45}^{+185}$ & $177_{-72}^{+321}$ & $?^{?+}$ & $\bar{B}^{0} \rightarrow K^{-}\left(\pi^{+} \chi_{c 1}\right)$ & Belle [265] (5.0), BABAR [266] (2.0) \\
\hline \multirow[t]{5}{*}{$Y(4260)$} & $4251 \pm 9$ & $120 \pm 12$ & $1^{--}$ & $e^{+} e^{-} \rightarrow(\pi \pi J / \psi)$ & BABAR $[270,271](8)$, CLEO $[272,273](11)$ \\
\hline & & & & & Belle [155, 264] (15), BES III [156] (np) \\
\hline & & & & $e^{+} e^{-} \rightarrow\left(f_{0}(980) J / \psi\right)$ & $B A B A R$ [271] (np), Belle [155] (np) \\
\hline & & & & $e^{+} e^{-} \rightarrow\left(\pi^{-} Z_{c}(3900)^{+}\right)$ & BES III [156] (8), Belle [155] (5.2) \\
\hline & & & & $e^{+} e^{-} \rightarrow(\gamma X(3872))$ & BES III [110] (5.3) \\
\hline$X(4274)$ & $4273_{-9}^{+19}$ & $56_{-16}^{+13}$ & $1^{++}$ & $B^{+} \rightarrow(J / \psi \phi) K^{+}$ & LHCb $[135,136](6.0)$, see Table 16 \\
\hline$(X(4350) ?)$ & $4350.6_{-5.1}^{+4.6}$ & $13_{-10}^{+18}$ & $0 / 2^{?+}$ & $e^{+} e^{-} \rightarrow e^{+} e^{-}(\phi \mathrm{J} / \psi)$ & Belle [274] (3.2) \\
\hline$Y(4360)$ & $4346.2 \pm 6.3$ & $102.3 \pm 9.9$ & $1^{--}$ & $e^{+} e^{-} \rightarrow\left(\pi^{+} \pi^{-} \psi(2 S)\right)$ & Belle $[267,275]$ (8), BABAR [276] (np) \\
\hline$P_{c}(4380)^{+}$ & $4380 \pm 30$ & $205 \pm 88$ & $3 / 2^{-}$ & $\Lambda_{b}^{0} \rightarrow K^{-}(J / \psi p)$ & LHCb [114] (9) \\
\hline$Y(4390)$ & $4391.6 \pm 6.4$ & $139.5 \pm 16.1$ & $1^{--}$ & $e^{+} e^{-} \rightarrow\left(\pi^{+} \pi^{-} h_{c}\right)$ & BES III [108] (10) \\
\hline
\end{tabular}

Table 9: Summary of quarkoniumlike mesons and pentaquarks. For charged states, the $C$-parity is given for the neutral members of the corresponding isotriplets. The states in parentheses are mere evidence of states, with low significance. The controversy on the $Y(4008)$ will be discussed in Section 8.4. Also, new data about the $Y(4260)$ by BES III [108] are not included in the average. The signature assignments in parentheses are just tentative, or deduced by the measurement of the charged partners. 


\begin{tabular}{|c|c|c|c|c|c|}
\hline State & $M(\mathrm{MeV})$ & $\Gamma(\mathrm{MeV})$ & $J^{P C}$ & Process (mode) & Experiment $(\# \sigma)$ \\
\hline \multirow[t]{3}{*}{$Z(4430)^{+}$} & $4478 \pm 17$ & $180 \pm 31$ & $1^{+-}$ & $\bar{B}^{0} \rightarrow K^{-}\left(\pi^{+} \psi(2 S)\right)$ & Belle $[277,278](6.4), B A B A R[279](2.4)$ \\
\hline & & & & & $\mathrm{LHCb}[280](13.9)$ \\
\hline & & & & $\bar{B}^{0} \rightarrow K^{-}\left(\pi^{+} J / \psi\right)$ & Belle [268] (4.0) \\
\hline$P_{c}(4450)^{+}$ & $4449.8 \pm 3.0$ & $39 \pm 20$ & $5 / 2^{+}$ & $\Lambda_{b}^{0} \rightarrow K^{-}(J / \psi p)$ & $\mathrm{LHCb}[114](12)$ \\
\hline$X(4500)$ & $4506_{-19}^{+16}$ & $92_{-29}^{+30}$ & $0^{++}$ & $B^{+} \rightarrow(J / \psi \phi) K^{+}$ & LHCb $[135,136](6.1)$, see Table 16 \\
\hline$Y(4630)$ & $4634_{-11}^{+9}$ & $92_{-32}^{+41}$ & $1^{--}$ & $e^{+} e^{-} \rightarrow\left(\Lambda_{c}^{+} \bar{\Lambda}_{c}^{-}\right)$ & Belle [281] (8.2) \\
\hline$Y(4660)$ & $4657 \pm 11$ & $70 \pm 11$ & $1^{--}$ & $e^{+} e^{-} \rightarrow\left(\pi^{+} \pi^{-} \psi(2 S)\right)$ & Belle $[267,275](5.8), B A B A R[276]$ (5) \\
\hline$X(4700)$ & $4704_{-26}^{+17}$ & $120_{-45}^{+52}$ & $0^{++}$ & $B^{+} \rightarrow(J / \psi \phi) K^{+}$ & LHCb $[135,136](5.6)$, see Table 16 \\
\hline$(X(5568) ?)$ & $5567.8_{-3.4}^{+3.0}$ & $21.9_{-6.9}^{+8.1}$ & $?$ & $p \bar{p} \rightarrow\left(B_{s}^{0} \pi^{ \pm}\right)+$All & $\mathrm{D} \varnothing[133]$ (3.9), LHCb [282] (not seen) \\
\hline \multirow[t]{3}{*}{$Z_{b}(10610)^{+}$} & $10607.2 \pm 2.0$ & $18.4 \pm 2.4$ & $1^{+-}$ & $\Upsilon(5 S) \rightarrow \pi(\pi \Upsilon(n S))$ & Belle [283] $(>10)$ \\
\hline & & & & $\Upsilon(5 S) \rightarrow \pi^{-}\left(\pi^{+} h_{b}(n P)\right)$ & Belle [283] (16) \\
\hline & & & & $\Upsilon(5 S) \rightarrow \pi^{-}\left(B \bar{B}^{*}\right)^{+}$ & Belle $[284,285](9.3)$ \\
\hline$Z_{b}(10610)^{0}$ & $10609 \pm 4 \pm 4$ & 18.4 (fixed) & $\left(1^{+-}\right)$ & $\Upsilon(5 S) \rightarrow \pi(\pi \Upsilon(n S))$ & Belle [286] (6.1) \\
\hline \multirow[t]{3}{*}{$Z_{b}(10650)^{+}$} & $10652.2 \pm 1.5$ & $11.5 \pm 2.2$ & $1^{+-}$ & $\Upsilon(5 S) \rightarrow \pi^{-}\left(\pi^{+} \Upsilon(n S)\right)$ & Belle $[283](>10)$ \\
\hline & & & & $\Upsilon(5 S) \rightarrow \pi^{-}\left(\pi^{+} h_{b}(n P)\right)$ & Belle [283] (16) \\
\hline & & & & $\Upsilon(5 S) \rightarrow \pi^{-}\left(B^{*} \bar{B}^{*}\right)^{+}$ & Belle $[284,285](8.1)$ \\
\hline
\end{tabular}

Table 10: (continued) Summary of quarkoniumlike mesons and pentaquarks. For charged states, the $C$-parity is given for the neutral members of the corresponding isotriplets. The states in parentheses are mere evidence of states, with low significance. The signature assignments in parentheses are just tentative, or deduced by the measurement of the charged partners.

- In double charmonium production, usually $e^{+} e^{-} \rightarrow J / \psi X$, which constrains $X$ to have $C=+$. This allows for a partial reconstruction of the $X$ state. The $B$-factories discovered the $X(3940)$ and the $X(4160)$ with this technique (Section 8.8).

- In $B$ decays, which allow $X$ to have any $J^{P C}$, albeit low values of the spin are preferred. Both exclusive and inclusive analyses can be performed. The $B$-factories provide a clean environment, but the huge amount of data collected at $\mathrm{LHCb}$ allow for very-high statistics, multidimensional analyses, in particular if no neutral particles are involved. The $X(3872)$ (Section 8.1), the $Z(4430)$ (Section 8.5) and the $[c s][\bar{c} \bar{s}]$ (Section 8.6) states have been discovered in $B$ decays.

- In $B_{c}$ and bottom baryon decays. LHCb is the main performer in these channels. The general-purpose detectors CMS and ATLAS can in principle look for these channels, but they suffer from the lack of particle identification, and a much larger background. These channel has lead to the discovery of the pentaquarks, see Section 8.7.

- In $\psi(n S), Y(4260)$ and $\Upsilon(n S)$ decays. The $Z_{c}$ s and $Z_{b}$ s states have been discovered in this way by BES III and Belle, respectively (see Section 8.2 and 8.3 ).

- In inclusive analyses at hadron colliders. Until now, only the $X(3872)$ and the $X(4140)$ has been observed inclusively. It is usually possible to separate the non-prompt (due to the decay of some long-lived meson or baryon) and prompt fractions. These analyses have been performed by CDF, D $\varnothing$, LHCb, CMS and ATLAS, which testifies for the growing interest the exotic charmonium physics has raised in the high energy physics community.

Although the CLEO collaboration officially no longer exists, a group based in Northwestern University still publishes analyses based on CLEO- $c$ data. We will quote their results as the NU group. 


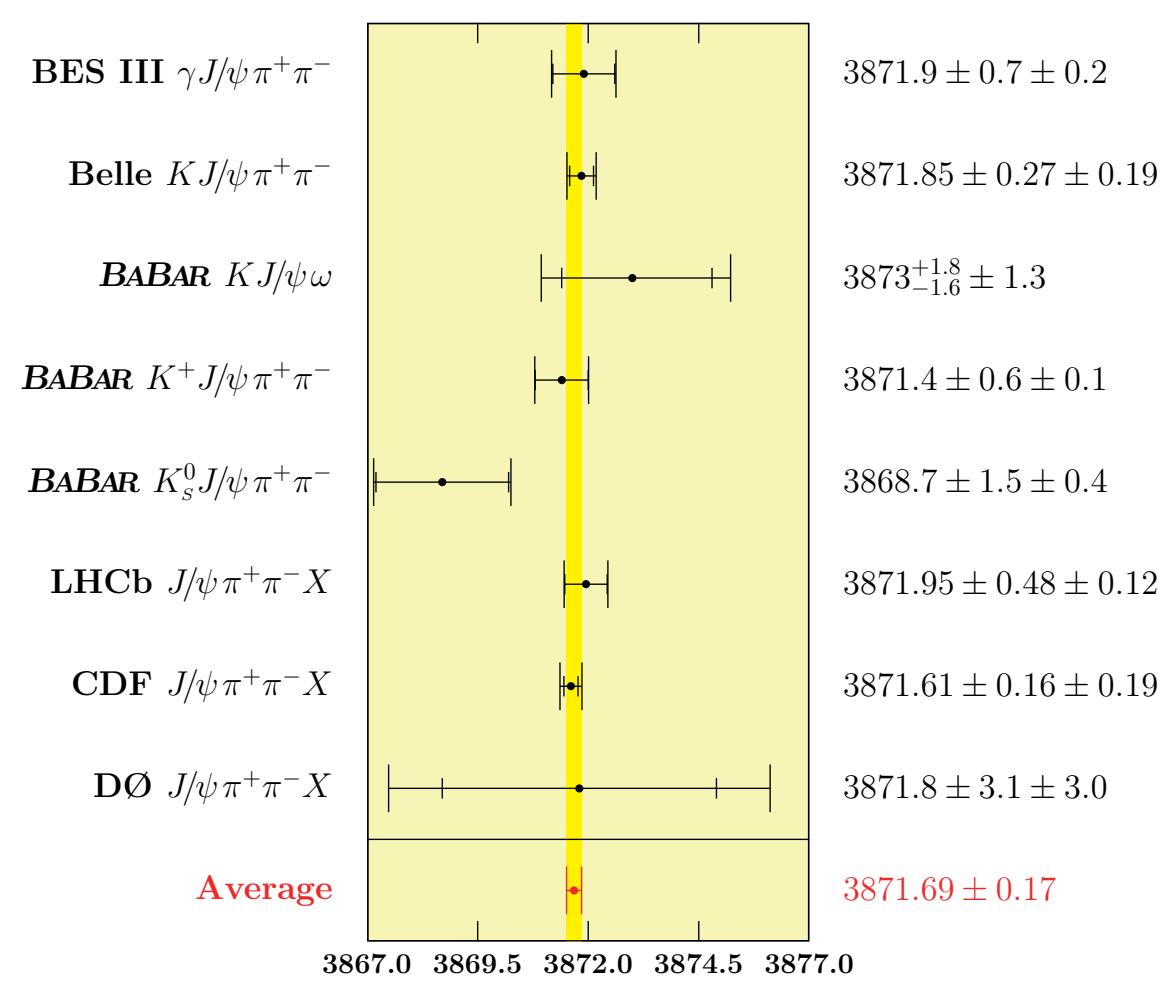

Figure 31: Averaged mass of the $X(3872)$, according to the measurements taken into account in the PDG [137].

The readers more interested in experimental details are invited to look at [2, 4, 6, 288, 289]. Prospects for future searches have been recently exposed in [290, 291].

\subsection{The $X(3872)$}

The discovery of an unexpected charmonium state in 2003 gave birth to the long saga of exotic quarkonia. The Belle collaboration announced the observation of a narrow resonance in the $B \rightarrow K\left(J / \psi \pi^{+} \pi^{-}\right)$channel, dubbed $X(3872)$ to stress its mysterious nature. ${ }^{47}$ In this decade, this state has been confirmed in many different production channels: in $B$ decays [293], in inclusive $p \bar{p}[46,294]$ and $p p$ collisions [45, 47], and more recently in the radiative decay of the $Y(4260)$ [110]. The measured mass is $M=3871.69 \pm 0.17 \mathrm{MeV}$ [137], while the best available upper bound on the width is given by $\Gamma<1.2 \mathrm{MeV}$ at $90 \%$ C.L. [295]. The mass of the state is some $100 \mathrm{MeV}$ lighter than expected for a $\chi_{c 1}(2 P)$ states, although this discrepancy is reduced in some calculations with unquenched light quarks [97]. The $\pi^{+} \pi^{-}$invariant mass distribution [27, 296] and angular analyses [297] showed that the $\pi^{+} \pi^{-}$amplitude is dominated by the $\rho$ meson. If this state were an ordinary charmonium, the transition via an isovector light meson should be highly suppressed. The size of the isospin breaking is indeed signaled by the ratio between the radiative decay width [137, 298-301], with respect to the isospin violating mode,

$$
\frac{\Gamma(X(3872) \rightarrow J / \psi \gamma)}{\Gamma\left(X(3872) \rightarrow J / \psi \rho\left(\rightarrow \pi^{+} \pi^{-}\right)\right)}=0.24 \pm 0.05
$$

\footnotetext{
${ }^{47}$ It is worth noticing that the Belle paper on the $X(3872)$ discovery [27] has $50 \%$ more citations than the one on the CP violation in the $B^{0}$ system [292], for which the $B$-factories were designed and financed. This should give an idea of the broad interest this new field piqued in the high energy physics community.
} 

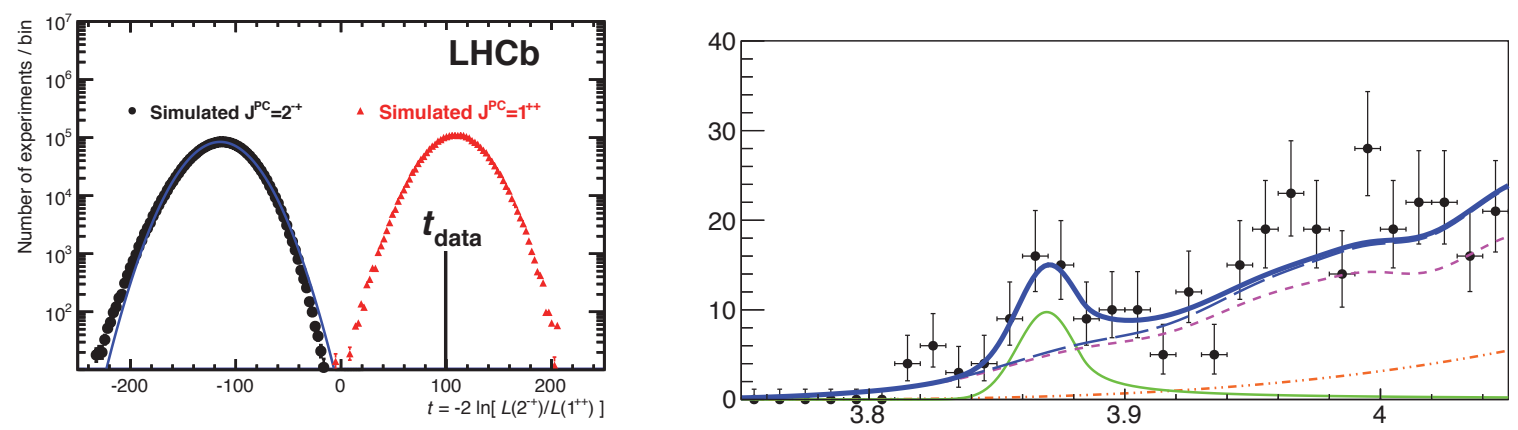

Figure 32: Left panel: Likelihood ratio test for the spin of the $X(3872)$, favoring the $1^{++}$assignment, by LHCb [308]. Right panel: mass projection of the $\psi(2 S) \gamma$ invariant mass, where the $X(3872)$ is evident, by LHCb [316].

to compare, for example, to an expected ratio of $O(10)$ if the $X(3872)$ were the $\chi_{c 1}(2 P)$ [302]. Finally, the ratio [259]

$$
\frac{\Gamma\left(X(3872) \rightarrow J / \psi \omega\left(\rightarrow \pi^{+} \pi^{-} \pi^{0}\right)\right)}{\Gamma\left(X(3872) \rightarrow J / \psi \rho\left(\rightarrow \pi^{+} \pi^{-}\right)\right)}=0.8 \pm 0.3,
$$

manifestly shows an isospin violation of $O(1)$.

The observation of the radiative decay, and the non-observation of $X(3872) \rightarrow \chi_{c 1} \gamma$ [27], established the $C=+$ assignment. For $J^{P}$, the first angular analyses of the $J / \psi \pi^{+} \pi^{-}$decay by Belle [303] and CDF [297] were able to rule out all but the $1^{++}$and $2^{-+}$assignments. The latter could not be excluded because of the two independent helicity amplitudes for $X\left(2^{-+}\right) \rightarrow J / \psi \rho$, which turn out in an additional complex parameter, hard to constrain in inclusive $X(3872)$ production. On the other hand, the axial assignment was preferred by theoretical models. The analysis of the $J / \psi \omega$ invariant mass distribution by BABAR [259] favored the $2^{-+}$hypothesis, and stimulated a discussion on its theoretical feasibility [289, 304-307]. The negative search of $X(3872)$ in $\gamma \gamma$ fusion at CLEO made the pseudotensor hypothesis unlikely. Later, Belle updated the angular analysis with the final dataset [295], still not being able to rule out $2^{-+}$. Finally, LHCb published two analyses of a large $B^{+} \rightarrow K^{+} X(3872)$ sample [308, 309], respectively restricting or not to the lowest available partial wave. These studies are based on an event-by-event likelihood ratio test of the two hypotheses on the full 5D angular distribution, and favor the $1^{++}$over $2^{-+}$at $8 \sigma$ level (Figure 32, left panel).

In Figure 31 we report the list of the mass measurements used for the PDG average. The current world average takes into account the $X(3872) \rightarrow J / \psi \rho, \omega$ decays. The mass coincides with the $D^{0} D^{* 0}$ threshold within errors, which gave birth to all the speculations about the molecular nature of this state - see Section 3. The most accurate estimate of the 'binding energy' is [29]

$$
B=M_{D}+M_{D^{*}}-M_{X}=(3 \pm 192) \mathrm{keV} .
$$

A precise determination of the mass, together with the precise determination of the width, will be able to constrain the loosely bound molecule hypothesis [17]. The mass observed in the $X(3872) \rightarrow D^{* 0} \bar{D}^{0}$ decay [310-312] is significantly higher, $M=(3873.8 \pm 0.5) \mathrm{MeV}$. The possibility that $X(3875) \rightarrow D^{* 0} \bar{D}^{0}$ and $X(3872) \rightarrow J / \psi \pi^{+} \pi^{-}$are distinct particles, for example the two almost-degenerate $[c u][\bar{c} \bar{u}]$ and $[c d][\bar{c} \bar{d}]$ tetraquarks, was discussed in [86]. Some papers [313-315] argued instead that, since the $D^{* 0}$ is in general off-shell, only the detailed study of the $\pi^{0} D^{0} \bar{D}^{0}$ and $\gamma D^{0} \bar{D}^{0}$ lineshapes can distinguish between a below- and above-threshold $X(3872)$. Moreover, in order to improve the resolution, the experimental analyses constrain the $D^{*}$ mass, and this yields to a reconstructed $X(3872)$ mass which is above threshold by construction. Because of these biases, this channel has been dropped from the mass averages in the PDG.

The measurement of radiative decays can be useful to constrain some of the available models. BABAR [300] and LHCb [316] observed a $X(3872)$ signal in the $\psi(2 S) \gamma$ final state (Figure 32, right panel), giving a relative branching 
fraction of

$$
\frac{\Gamma(X(3872) \rightarrow \psi(2 S) \gamma)}{\Gamma(X(3872) \rightarrow J / \psi \gamma)}=2.6 \pm 0.6
$$

On the other hand, Belle saw no significant signal in this channel, and put a $<2.190 \%$ C.L. upper limit on the ratio. In the original molecular model proposed by Swanson, the radiative transition to $\psi(2 S)$ was predicted to be suppressed [1, 317]. In an analysis based on NREFT (see Section 3.5), Hanhart et al. claim that the radiative decays depend on the short-range structure of the molecule, and give up on predicting the ratio, thus 'reconciling' the molecular hypothesis with experimental data. A calculation by Braaten [318] based on universal low energy scattering theory (see Section $3.2)$, predicted

$$
\Gamma(X(3872) \rightarrow p \bar{p})=\left(\frac{\Lambda}{m_{\pi}}\right)^{2} \sqrt{\frac{B}{600 \mathrm{keV}}} \times 28 \mathrm{eV}
$$

where $\Lambda$ is the scale up to which one can neglect the effective range of the interaction (say $\sim 3 m_{\pi}$ ), and $B$ the molecule binding energy. Using $B=190 \mathrm{keV}$ (the available upper limit) and $\Gamma \gtrsim \Gamma\left(D^{* 0}\right) \sim 70 \mathrm{keV}$, one gets $\mathcal{B}(X \rightarrow p \bar{p}) \lesssim$ $2 \times 10^{-3}$, which coincides with the experimental bound by LHCb $[319,320]$. Further improvement of the precision on this branching ratio, together with a better determination of the binding energy and width of the $X(3872)$ are needed to constrain the molecular hypothesis. The decay $Y(4260) \rightarrow \gamma X(3872)$ has been observed by BES III [110]. We will discuss this channel in the Section 8.4.

Other production mechanisms like $B^{0} \rightarrow K^{+} \pi^{-} X(3872)$ have also been studied. Such decays are seen [321, 322], but the non-resonant $K \pi$ dominates. This is in contrast to ordinary charmonium states, where the $B \rightarrow K^{*} c \bar{c}$ and $B \rightarrow K c \bar{c}$ branching fractions are comparable, and $K^{*}$ dominates over non-resonant $K \pi$. Also, a preliminary analysis by COMPASS shows the production of $\psi(2 S)$ and $X(3872)$ in the $\mu^{+} N \rightarrow \mu^{+}\left(J / \psi \pi^{+} \pi^{-}\right) \pi^{ \pm} N^{\prime}$ process [323]. The ratio of events for the two states is $N(X(3872)) / N(\psi(2 S))=0.9 \pm 0.4$. If the production mechanism is assumed to be the same (a vector meson dominated virtual photon), this ratio equals $\Gamma\left(X(3872) \rightarrow J / \psi \pi^{+} \pi^{-}\right) \times \mathcal{B}(X(3872) \rightarrow$ $\left.J / \psi \pi^{+} \pi^{-}\right) / \Gamma\left(\psi(2 S) \rightarrow J / \psi \pi^{+} \pi^{-}\right) \times \mathcal{B}\left(\psi(2 S) \rightarrow J / \psi \pi^{+} \pi^{-}\right)$, and one might use this to constrain the total width of the $X(3872)$.

In a decade of experimental activity, the $X(3872)$ has been searched in a plethora of different final states, some of which allowed us to establish the correct quantum numbers. In most of these analyses, the $X(3872)$ is produced in $B$ decays, so that it is not possible to disentangle the production branching fraction $\mathcal{B}(B \rightarrow K X)$ from the final $\mathcal{B}(X \rightarrow f)$. These factors cancels in the ratio, but some model have predictions for the absolute branching fraction. An inclusive $B A B A R$ analysis of $B^{+} \rightarrow K^{+}(c \bar{c})$ states provides an upper limit for the production branching fraction, $\mathcal{B}(B \rightarrow K X(3872))<3.2 \times 10^{-4}$ at $90 \%$ C.L. [324]. Combining the likelihood from the measurements of the product branching fractions in the observed channels, the $B \rightarrow X(3872) K$ upper limit, the $X(3872)$ width distribution [155], we extract the likelihood for the absolute $X(3872)$ branching fractions and the widths in each of the decay modes with a bayesian procedure. We then use these distributions to set limits on the not observed channels. The full shape of the experimental likelihoods was used whenever available, while gaussian errors and poissonian counting distributions have been assumed elsewhere. We summarize the results in Table 11 and 12. The plot of the likelihoods and the code are available at [325].

The searches for neutral and charged partner states of the $X(3872)$ have been motivated by the predictions of the tetraquark model. For example, Ref. [7] proposed that the $X$ states produced in $B^{+}$and $B^{0}$ decays were different. If so, the masses of the two $X$ should differ by a few MeVs. The analyses by BABAR [327, 328] and Belle [295, 321] distinguish the two samples, and give for the mass difference $M\left(X \mid B^{+} \rightarrow K^{+} X\right)-M\left(X \mid B^{0} \rightarrow K^{0} X\right)=(0.2 \pm 0.8) \mathrm{MeV}$, and for the ratio of product branching fractions

$$
\frac{\mathcal{B}\left(B^{0} \rightarrow K^{0} X\right) \times \mathcal{B}\left(X \rightarrow \pi^{+} \pi^{-} J / \psi\right)}{\mathcal{B}\left(B^{+} \rightarrow K^{+} X\right) \times \mathcal{B}\left(X \rightarrow \pi^{+} \pi^{-} J / \psi\right)}=0.47 \pm 0.13
$$

Similarly, the inclusive analysis by CDF [329], of the $J / \psi \pi^{+} \pi^{-}$spectrum, gave no evidence for a second neutral state, setting an upper limit on the mass difference of $3.6 \mathrm{MeV}$ at the $95 \%$ C.L.. The charged partners have been searched at 

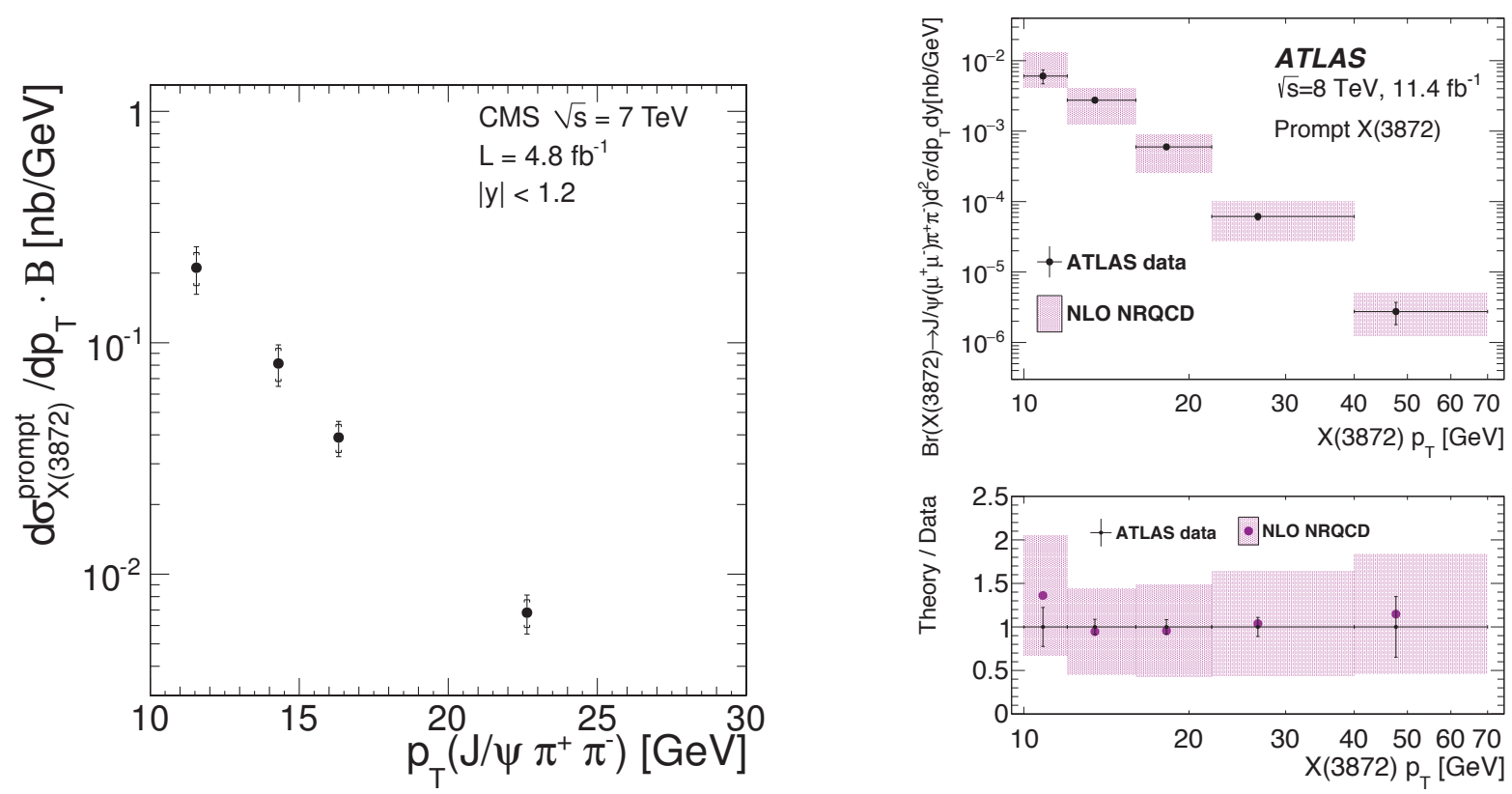

Figure 33: Measured differential cross section for prompt $X(3872)$ production times branching fraction $\mathcal{B}\left(X(3872) \rightarrow J / \psi \pi^{+} \pi^{-}\right)$as a function of $p_{T}$, from CMS [45] (left panel), and ATLAS [326] (right panel).

the $B$-factories [295, 330], but no evidence is seen. The strictest limits on the product branching fractions are

$$
\begin{aligned}
& \mathcal{B}\left(\bar{B}^{0} \rightarrow K^{-} X^{+}\right) \times \mathcal{B}\left(X^{+} \rightarrow \rho^{+} J / \psi\right)<4.2 \times 10^{-6}, \\
& \mathcal{B}\left(B^{+} \rightarrow K^{0} X^{+}\right) \times \mathcal{B}\left(X^{+} \rightarrow \rho^{+} J / \psi\right)<6.1 \times 10^{-6},
\end{aligned}
$$

but they are not so severe with respect to the neutral channel [295, 328], $\mathcal{B}\left(B^{+} \rightarrow K^{+} X\right) \times \mathcal{B}\left(X \rightarrow \rho^{0} J / \psi\right)=(8.6 \pm$ $0.81) \times 10^{-6}$. Recently, Voloshin proposed that these charged states could mix with the $Z_{c}(3900)$ because of isospin breaking, suggesting to resolve two close peaks in the $Y(4260) \rightarrow \pi^{+}\left(D D^{*}\right)^{-}$process [331].

We conclude this section on the $X(3872)$ with the inclusive production at hadron colliders, which has been extensively studied in the literature (see Appendix A). The prompt production has been studied at CDF [332] and CMS [45], giving

$$
\begin{aligned}
& \frac{\sigma^{\mathrm{prompt}}(p \bar{p} \rightarrow X(3872)+\text { all })}{\sigma(p \bar{p} \rightarrow X(3872)+\text { all })}=(83.9 \pm 4.9 \pm 2.0) \% \text { at } \sqrt{s}=1.96 \mathrm{TeV}, \\
& \frac{\sigma^{\text {prompt }}(p p \rightarrow X(3872)+\text { all })}{\sigma(p p \rightarrow X(3872)+\text { all })}=(73.7 \pm 2.3 \pm 1.6) \% \text { at } \sqrt{s}=7 \mathrm{TeV}
\end{aligned}
$$

The same measurement is not explicitly presented in the CDF note, but Bignamini et al. estimated [31]:

$$
\sigma^{\text {prompt }}(p p \rightarrow X(3872)+\text { all }) \times \mathcal{B}\left(X(3872) \rightarrow J / \psi \pi^{+} \pi^{-}\right)=(3.1 \pm 0.7) \mathrm{nb} \text { at } \sqrt{s}=1.96 \mathrm{TeV}
$$

CMS published the value for the integrated prompt production cross section, $\sigma^{\text {prompt }}(p p \rightarrow X(3872)+$ all $) \times$ $\mathcal{B}\left(X(3872) \rightarrow J / \psi \pi^{+} \pi^{-}\right)=(1.06 \pm 0.11 \pm 0.15) \mathrm{nb}$ in the region $10<p_{\perp}<30 \mathrm{GeV}$ and $|y|<1.2$, at $\sqrt{s}=7 \mathrm{TeV}$. The differential cross section as a function of $p_{\perp}$ is shown in Figure 33, left panel. LHCb measured the inclusive 


\begin{tabular}{|c|c|c|c|c|c|}
\hline \multirow{2}{*}{$\frac{B \text { decay mode }}{K^{+} X}$} & \multirow{2}{*}{$\begin{array}{l}X \text { decay mode } \\
X \rightarrow \pi \pi J / \psi\end{array}$} & \multicolumn{2}{|c|}{ product branching fraction $\left(\times 10^{5}\right)$} & \multirow{2}{*}{$\frac{B_{\text {fit }}(\%)}{7.3_{-2.3}^{+2.8}}$} & \multirow{2}{*}{$\begin{array}{c}R_{\text {fit }} \\
1\end{array}$} \\
\hline & & $0.86 \pm 0.08$ & average & & \\
\hline & & $0.84 \pm 0.15 \pm 0.07$ & BABAR [328] & & \\
\hline & & $0.86 \pm 0.08 \pm 0.05$ & Belle [295] & & \\
\hline \multirow[t]{3}{*}{$K^{0} X$} & $X \rightarrow \pi \pi J / \psi$ & $0.41 \pm 0.11$ & average & & \\
\hline & & $0.35 \pm 0.19 \pm 0.04$ & BABAR [328] & & \\
\hline & & $0.43 \pm 0.12 \pm 0.04$ & Belle [295] & & \\
\hline$\left(K^{+} \pi^{-}\right)_{N R} X$ & $X \rightarrow \pi \pi J / \psi$ & $0.79 \pm 0.13 \pm 0.04$ & Belle [322] & & \\
\hline$K^{* 0} X$ & $X \rightarrow \pi \pi J / \psi$ & $R^{\prime}=(34 \pm 9 \pm 2) \%$ & Belle [322] & & \\
\hline$\left(K^{0} \pi^{+}\right)_{N R} X$ & $X \rightarrow \pi \pi J / \psi$ & $1.06 \pm 0.30 \pm 0.09$ & Belle [322] & & \\
\hline$K X$ & $X \rightarrow \omega J / \psi$ & $R=0.8 \pm 0.3$ & BABAR [259] & $5.3 \pm 2.8$ & $0.65_{-0.20}^{+0.41}$ \\
\hline$K^{+} X$ & & $0.6 \pm 0.2 \pm 0.1$ & $B A B A R$ [259] & & \\
\hline$K^{0} X$ & & $0.6 \pm 0.3 \pm 0.1$ & $B A B A R$ [259] & & \\
\hline$K X$ & $X \rightarrow \pi \pi \pi^{0} J / \psi$ & $R=1.0 \pm 0.4 \pm 0.3$ & Belle [298] & & \\
\hline \multirow[t]{3}{*}{$K^{+} X$} & $X \rightarrow D^{* 0} \bar{D}^{0}$ & $8.5 \pm 2.6$ & average & $66_{-12}^{+13}$ & $8.2_{-1.8}^{+1.9}$ \\
\hline & & $16.7 \pm 3.6 \pm 4.7$ & $B A B A R[311]$ & & \\
\hline & & $7.7 \pm 1.6 \pm 1.0$ & Belle [312] & & \\
\hline \multirow[t]{3}{*}{$K^{0} X$} & $X \rightarrow D^{* 0} \bar{D}^{0}$ & $12 \pm 4$ & average & & \\
\hline & & $22 \pm 10 \pm 4$ & BABAR [311] & & \\
\hline & & $9.7 \pm 4.6 \pm 1.3$ & Belle [312] & & \\
\hline$K^{+} X$ & $X \rightarrow \gamma J / \psi$ & $0.202 \pm 0.038$ & average & $1.73 \pm 0.68$ & $0.24 \pm 0.06$ \\
\hline \multirow[t]{2}{*}{$K^{+} X$} & & $0.28 \pm 0.08 \pm 0.01$ & BABAR [300] & & \\
\hline & & $0.178_{-0.044}^{+0.048} \pm 0.012$ & Belle [301] & & \\
\hline \multirow[t]{2}{*}{$K^{0} X$} & & $0.26 \pm 0.18 \pm 0.02$ & BABAR [300] & & \\
\hline & & $0.124_{-0.061}^{+0.076} \pm 0.011$ & Belle [301] & & \\
\hline$K^{+} X$ & $X \rightarrow \gamma \psi(2 S)$ & $0.44 \pm 0.12$ & BABAR [300] & $3.8 \pm 1.8$ & $0.51 \pm 0.15$ \\
\hline \multirow[t]{3}{*}{$K^{+} X$} & & $0.95 \pm 0.27 \pm 0.06$ & BABAR [300] & \multirow{3}{*}{\multicolumn{2}{|c|}{$R_{\text {fit }}^{\prime \prime}=2.25_{-0.65}^{+0.55}$}} \\
\hline & & $0.083_{-0.183}^{+0.198} \pm 0.044$ & Belle [301] & & \\
\hline & & $R^{\prime \prime}=2.46 \pm 0.64 \pm 0.29$ & LHCb [316] & & \\
\hline \multirow[t]{2}{*}{$K^{0} X$} & & $1.14 \pm 0.55 \pm 0.10$ & $B A B A R$ [300] & & \\
\hline & & $0.112_{-0.290}^{+0.357} \pm 0.057$ & Belle [301] & & \\
\hline
\end{tabular}

Table 11: Measured $X(3872)$ product branching fractions, separated by production and decay channel. The last two columns report the results in terms of absolute $X(3872)$ branching fraction $\left(B_{\mathrm{fit}}\right)$ and in terms of the branching fraction normalized to $J / \psi \pi \pi\left(R_{\mathrm{fit}}\right)$ as obtained from the global likelihood fit described in the text. For non-zero measurements we report the mean value, and the $68 \%$ C.L. range in form of asymmetric errors. The limits are provided at $90 \%$ C.L.. The details of the fit are discussed in the text, the code is available on [325]. The $X(3872) \rightarrow$ $\pi \pi \pi^{0} J / \psi$ is dominated by $\omega J / \psi$, but no limits on the non-resonant $\pi \pi \pi^{0} J / \psi$ component have been set. The ratio $R^{\prime}$ given by Belle [322] is $\left.\left.\mathcal{B}\left(B^{0} \rightarrow X K^{* 0}(892)\right) \times \mathcal{B}\left(K^{* 0}(892) \rightarrow K^{+} \pi^{-}\right)\right) / \mathcal{B}\left(B^{0} \rightarrow X K^{+} \pi^{-}\right)\right)$. The ratio $R^{\prime \prime}$ is $\mathcal{B}(X(3872) \rightarrow \psi(2 S) \gamma) / \mathcal{B}(X(3872) \rightarrow J / \psi \gamma)$. 


\begin{tabular}{llllcc}
\hline \hline$B$ decay mode & $X$ decay mode & \multicolumn{2}{c}{ product branching fraction $\left(\times 10^{5}\right)$} & $B_{\text {fit }}$ & $R_{\text {fit }}$ \\
\hline$K^{+} X$ & $X \rightarrow p \bar{p}$ & $<9.6 \times 10^{-4}$ & LHCb [319,320] & $<1.7 \times 10^{-4}$ & $<2.4 \times 10^{-3}$ \\
\hline$K^{+} X$ & $X \rightarrow \gamma \chi_{c 1} \S$ & $<9.6 \times 10^{-3}$ & Belle [333] & $<1.0 \times 10^{-3}$ & $<0.014$ \\
$K^{+} X$ & $X \rightarrow \gamma \chi_{c 2^{\S}}$ & $<0.016$ & Belle [333] & $<1.7 \times 10^{-3}$ & $<0.024$ \\
$K X$ & $X \rightarrow \gamma \gamma^{\S}$ & $<4.5 \times 10^{-3}$ & Belle [334] & $<4.5 \times 10^{-4}$ & $<6.6 \times 10^{-3}$ \\
$K X$ & $X \rightarrow \eta J / \psi^{\S}$ & $<1.05$ & BABAR [335] & $<0.11$ & $<1.6$ \\
$K^{+} X$ & $X \rightarrow D^{0} \bar{D}^{0 \S}$ & $<6 \times 10^{-5}$ & Belle [336] & $<0.29$ & $<4$ \\
$K^{+} X$ & $X \rightarrow D^{+} D^{-\S}$ & $<4 \times 10^{-5}$ & Belle [336] & $<0.25$ & $<3.6$ \\
\hline \hline Other modes & & Fraction/Width & & & - \\
\hline$\gamma \gamma \rightarrow X^{\S}$ & $X \rightarrow J / \psi \pi \pi$ & $\Gamma_{\gamma \gamma} \times \mathcal{B}_{\psi \pi \pi}<12.9 \mathrm{eV}$ & CLEO [337] & & $\Gamma_{e e}<2.34 \mathrm{eV}$ \\
$e^{+} e^{-} \rightarrow X$ & $X \rightarrow J / \psi \pi \pi$ & $\Gamma_{e e} \times \mathcal{B}_{\psi \pi \pi}<0.13 \mathrm{eV}$ & BES III [338] & & - \\
$\mu N \rightarrow \mu N^{\prime} \pi X$ & $X \rightarrow J / \psi \pi \pi$ & $R^{\prime \prime \prime}=0.9 \pm 0.4$ & COMPASS [323] & & $<$ \\
\hline \hline
\end{tabular}

Table 12: Continuation of Table 11. Measured $X(3872)$ product branching fractions, separated by production and decay channel. The last two columns report the results in terms of absolute $X(3872)$ branching fraction $\left(B_{f i t}\right)$ and in terms of the branching fraction normalized to $J / \psi \pi \pi\left(R_{f i t}\right)$ as obtained from the global likelihood fit described in the text. For non-zero measurements we report the mean value, and the $68 \%$ C.L. range in form of asymmetric errors. The limits are provided at 90\% C.L.. The details of the fit are discussed in the text, the code is available on [325]. Given the preliminary nature of the result by COMPASS [323], the ratio $R^{\prime \prime \prime}=\frac{\mathcal{B}(X \rightarrow \psi \pi \pi)^{2} \Gamma_{X}}{\mathcal{B}(\psi(2 S) \rightarrow \psi \pi \pi)^{2} \Gamma_{\psi}(2 S)}$ is not used to constrain the fit. The channels with $\S$ are forbidden by the signature $J^{P C}=1^{++}$.

cross section (without separating the prompt contribution) to be $\sigma^{\text {incl }}\left(p p \rightarrow B_{c}+\right.$ all $) \times \mathcal{B}\left(B_{c} \rightarrow X(3872)+\right.$ all $)=$ $(5.4 \pm 1.3 \pm 0.8) \mathrm{nb}$ in the region $5<p_{\perp}<20 \mathrm{GeV}$ and $2.5<y<4.5$, at $\sqrt{s}=7 \mathrm{TeV}$ [47]

Very recently, ATLAS published a preliminary study on the prompt production of the $X(3872)$. In the $|y|<0.75$, $10<p_{\perp}<70 \mathrm{GeV}$ region, they measured the prompt and non-prompt fractions with respect to the $\psi(2 S)$, and distinguished between long-lived (i.e. from $B, B_{s}$ and $\Lambda_{b}$ decays), and short-lived (i.e. from $B_{c}$ decay) non-prompt production

$$
\begin{aligned}
\frac{\mathcal{B}\left(B, B_{s}, \Lambda_{b} \rightarrow X(3872)+\mathrm{All}\right) \times \mathcal{B}\left(X(3872) \rightarrow J / \psi \pi^{+} \pi^{-}\right)}{\mathcal{B}\left(B, B_{s}, \Lambda_{b} \rightarrow \psi(2 S)+\mathrm{All}\right) \times \mathcal{B}\left(\psi(2 S) \rightarrow J / \psi \pi^{+} \pi^{-}\right)} & =(3.57 \pm 0.33 \pm 0.11) \% \\
\frac{\sigma\left(p p \rightarrow B_{c}+\text { all }\right) \times \mathcal{B}\left(B_{c} \rightarrow X(3872)+\text { all }\right)}{\sigma(p p \rightarrow X(3872) \text { non-prompt }+ \text { all })} & =(25 \pm 13 \pm 2 \pm 5) \%
\end{aligned}
$$

We report the differential cross section as a function of $p_{\perp}$, in Figure 33, right panel.

\subsection{The $Z_{c}(3900)$ and $Z_{c}^{\prime}(4020)$}

The discovery of two charged axial states in the $X(3872)$ region renewed the interest into the exotic hadron spectroscopy. We have discussed that the tetraquark model predicts two $J^{P C}=1^{+-}$states. Similarly, molecule with the same signature are expected to form at the $D D^{*}$ and $D^{*} D^{*}$ threshold. BES III [156] and Belle [155] observed an intense peak in the $J / \psi \pi^{+}$invariant mass, while studying the $Y(4260) \rightarrow J / \psi \pi^{+} \pi^{-}$channel $^{48}$ (Figure 34); BES III

\footnotetext{
${ }^{48}$ Some contamination from continuum events $e^{+} e^{-} \rightarrow \gamma^{*} \rightarrow Z_{c}(3900)^{+} \pi^{-}$can be present, and an energy dependent analysis is needed to enlighten on this.
} 

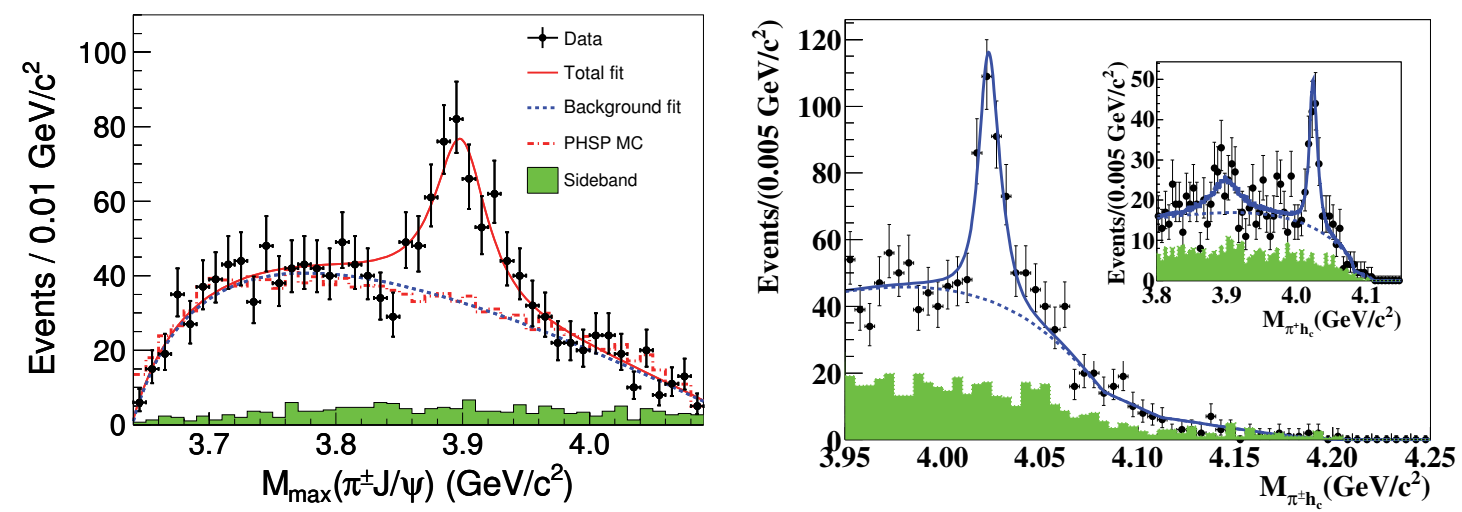

Figure 34: Left panel: Distributions of $M_{\max }\left(J / \psi \pi^{ \pm}\right)$, i.e. the larger one of the two $M\left(J / \psi \pi^{ \pm}\right)$in each event, according to BES III [156] (left) in the $Y(4260) \rightarrow J / \psi \pi^{+} \pi^{-}$decay. The red solid curve is the result of the fit, the blue dotted curve is the background component, the green histogram shows the normalized $J / \psi$ sideband events. Right panel: fits to the $M\left(h_{c} \pi\right)$ distributions by BES III [161]; the inset shows the sum fits if allowing for an additional $Z_{c}(3900)$ resonance.
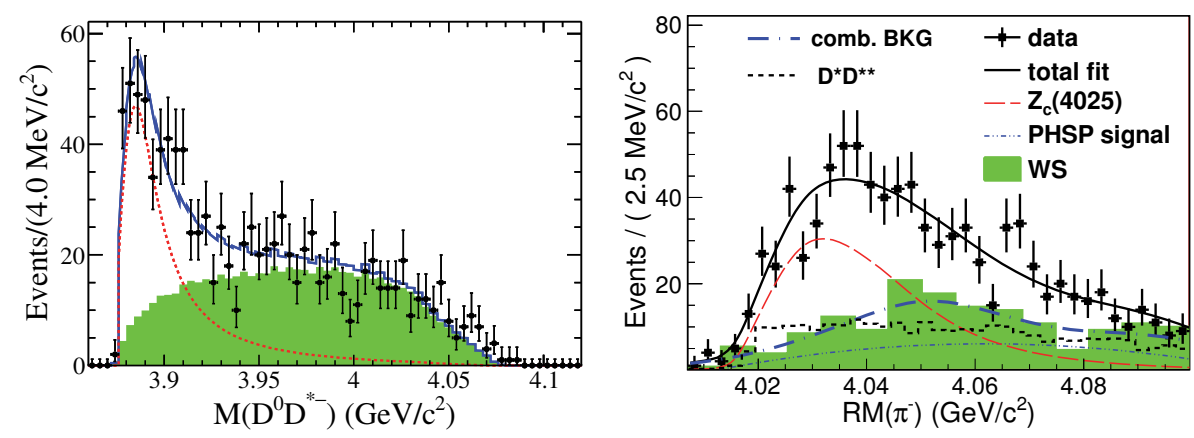

Figure 35: Left panel: Invariant mass distributions of $D^{0} D^{*-}$, in the BES III double tag analysis [251]. The solid curve is the result of the fit, the blue dotted curve is the background component. Right panel: Unbinned maximum likelihood fit to the $\pi$ recoil mass spectrum, in the $e^{+} e^{-} \rightarrow\left(D^{*} \bar{D}^{*}\right)^{+} \pi^{-}$ analysis by BES III [254].

by analyzing data at the $Y(4260)$ peak, and Belle studying events associated with an undetected ISR photon (for more details on the ISR physics, see Section 8.4).

The minimal quark content for such a state is $c \bar{c} u \bar{d}$, thus it is manifestly exotic. The averaged measured mass is $M=(3884.4 \pm 1.6) \mathrm{MeV}$, roughly $10 \mathrm{MeV}$ above the $D D^{*}$ threshold, and the width is $\Gamma=(27.9 \pm 2.7) \mathrm{MeV}$. This state was dubbed $Z_{c}(3900)$. The average production branching fractions is

$$
\frac{\mathcal{B}\left(Y(4260) \rightarrow Z_{c}(3900)^{+} \pi^{-}\right) \times \mathcal{B}\left(Z_{c}(3900)^{+} \rightarrow J / \psi \pi^{+}\right)}{\mathcal{B}\left(Y(4260) \rightarrow J / \psi \pi^{+} \pi^{-}\right)}=(22.4 \pm 3.1) \% .
$$

Soon after, BES III observed a similar signal in $Y(4260) \rightarrow\left(D \bar{D}^{*}\right)^{+} \pi^{-}$, both with single [159] and double tag [251] techniques (Figure 35). The signature $J^{P}=1^{+}$is favored by angular distributions, and the relative branching ratio is

$$
\frac{\mathcal{B}\left(Z_{c}(3900) \rightarrow D \bar{D}^{*}\right)}{\mathcal{B}\left(Z_{c}(3900) \rightarrow J / \psi \pi\right)}=7.7 \pm 1.3 \pm 2.8
$$

An analysis of CLEO- $c$ data by the NU group [252] confirmed the $Z_{c}(3900)$ in the decay of the $\psi(4160)$, and 
found a hint of a neutral $Z_{c}(3900)^{0}$ decaying to $J / \psi \pi^{0}$. BES III confirmed this neutral state, in both $J / \psi \pi^{0}$ and $\left(\bar{D} D^{*}\right)^{0}$ channels $[253,339]$, thus establishing the complete isospin triplet. This channel fixes the $C=-, G=+$ assignment.

The $Z_{c}$ (3900) has been searched with no luck in the OZI-suppressed $e^{+} e^{-} \rightarrow\left(\omega \pi^{+} \pi^{-}\right)$channel by BES III [340], in the $B \rightarrow\left(J / \psi \pi^{+}\right) K$ channel by Belle [268], and in photoproduction $\mu^{+} N \rightarrow \mu^{+}\left(J / \psi \pi^{+}\right) N^{\prime}$ at COMPASS [341].

A close sibling, dubbed $Z_{c}^{\prime}(4020)$, has been found by BES III in $e^{+} e^{-} \rightarrow\left(D^{*+} \bar{D}^{* 0}\right) \pi^{-}[254]$ and $e^{+} e^{-} \rightarrow\left(h_{c} \pi^{+}\right) \pi^{-}$ [161], with mass $M=(4023.9 \pm 2.4) \mathrm{MeV}$, slightly above the $D^{*} D^{*}$ threshold, and width $\Gamma=(10 \pm 6) \mathrm{MeV}^{49}$. The quantum numbers are $J^{P C}=1^{+-}$as well. There is also evidence for the neutral partner $Z_{c}^{\prime}(4020)^{0}$, in both closed and open charm channels $[255,256]$.

The $Z_{c}(3900)$ is also searched in the $h_{c} \pi$ final state [161], and a bump occurs at $2.1 \sigma$ level, thus not statistically significant. Similarly, no $Z_{c}^{\prime}(4020)$ peak is visible in the $J / \psi \pi$ spectrum [87]. This has to be compared with the $Z_{b}^{(\prime)}$ system, where both states appear in the same final states (see Section 8.3). This fact is of particular interest to constrain the NREFT molecular models (Section 3.5), which in general favor the decay into $h_{c}$ with respect to $J / \psi[54,55,57]$ : more statistics is needed to enlighten on this.

\subsection{The bottomonium system: $Z_{b}(10610)$ and $Z_{b}^{\prime}(10650)$, and search for $X_{b}$ and $Y_{b}$}

Heavy quark flavor symmetry suggests that a heavy replica of the $Z_{c}(3900)$ and $Z_{c}^{\prime}(4020)$ system has to occur in the bottomonium sector. Indeed, Belle observed two narrow resonances in the $\Upsilon(5 S) \rightarrow\left(\Upsilon(n S) \pi^{+}\right) \pi^{-}$and $\Upsilon(5 S) \rightarrow$ $\left(h_{b}(n P) \pi^{+}\right) \pi^{-}$channels [283]. A resonant intermediate structure explains both the anomalously high rate for $\Upsilon(5 S) \rightarrow$ $\Upsilon(n S) \pi^{+} \pi^{-}$(the partial widths are about two orders of magnitude larger than typical width for dipion transitions among the lower $(n S)$ states) [342], and the heavy quark spin symmetry violation in the decay into a $h_{b}$ [343].

We report the result of the Dalitz plot fits in Table 13. All the studied channels show the presence of two charged resonances, dubbed $Z_{b}(10610)$ and $Z_{b}^{\prime}(10650)$, with compatible masses and widths. The one-dimensional invariant mass projections for events in each $\Upsilon(n S)$ and $h_{b}(n P)$ signal region are shown in Figure 36. The averaged masses and widths are $M=(10607.2 \pm 2.0) \mathrm{MeV}, \Gamma=(18.4 \pm 2.4) \mathrm{MeV}$, and $M^{\prime}=(10652.2 \pm 1.5) \mathrm{MeV}, \Gamma^{\prime}=(11.5 \pm 2.2) \mathrm{MeV}$, respectively. The $Z_{b}(10610)$ production rate is similar to that of the $Z_{b}^{\prime}(10650)$ for each of the five decay channels. The fit shows that the relative phase between the two resonances is zero for the final states with the $\Upsilon(n S)$, and $180^{\circ}$ for the final states with $h_{b}(n P)$, as expected according to both the tetraquark [344], and the molecular model [48].

\begin{tabular}{|c|c|c|c|c|c|}
\hline Final state & $\Upsilon(1 S) \pi^{+} \pi^{-}$ & $\Upsilon(2 S) \pi^{+} \pi^{-}$ & $\Upsilon(3 S) \pi^{+} \pi^{-}$ & $h_{b}(1 P) \pi^{+} \pi^{-}$ & $h_{b}(2 P) \pi^{+} \pi^{-}$ \\
\hline$M\left[Z_{b}(10610)\right], \mathrm{MeV}$ & $10611 \pm 4 \pm 3$ & $10609 \pm 2 \pm 3$ & $10608 \pm 2 \pm 3$ & $10605 \pm 2_{-1}^{+3}$ & $10599_{-3-4}^{+6+5}$ \\
\hline$\Gamma\left[Z_{b}(10610)\right], \mathrm{MeV}$ & $22.3 \pm 7.7_{-4.0}^{+3.0}$ & $24.2 \pm 3.1_{-3.0}^{+2.0}$ & $17.6 \pm 3.0 \pm 3.0$ & $11.4_{-3.9-1.2}^{+4.5+2.1}$ & $13_{-8-7}^{+10+9}$ \\
\hline$M\left[Z_{b}(10650)\right], \mathrm{MeV}$ & $10657 \pm 6 \pm 3$ & $10651 \pm 2 \pm 3$ & $10652 \pm 1 \pm 2$ & $10654 \pm 3_{-2}^{+1}$ & $10651_{-3-2}^{+2+3}$ \\
\hline$\Gamma\left[Z_{b}(10650)\right], \mathrm{MeV}$ & $16.3 \pm 9.8_{-2.0}^{+6.0}$ & $13.3 \pm 3.3_{-3.0}^{+4.0}$ & $8.4 \pm 2.0 \pm 2.0$ & $20.9_{-4.7-5.7}^{+5.4+2.1}$ & $19 \pm 7_{-7}^{+11}$ \\
\hline Rel. normalization & $0.57 \pm 0.21_{-0.04}^{+0.19}$ & $0.86 \pm 0.11_{-0.10}^{+0.04}$ & $0.96 \pm 0.14_{-0.05}^{+0.08}$ & $1.39 \pm 0.37_{-0.15}^{+0.05}$ & $1.6_{-0.4-0.6}^{+0.6+0.4}$ \\
\hline Rel. phase, degrees & $58 \pm 43_{-9}^{+4}$ & $-13 \pm 13_{-8}^{+17}$ & $-9 \pm 19_{-26}^{+11}$ & $187_{-57-12}^{+44+3}$ & $181_{-105-109}^{+65+74}$ \\
\hline
\end{tabular}

Table 13: Comparison of results on $Z_{b}(10610)$ and $Z_{b}^{\prime}(10650)$ parameters obtained from $\Upsilon(5 S) \rightarrow \Upsilon(n S) \pi^{+} \pi^{-}$and $\Upsilon(5 S) \rightarrow h_{b}(n P) \pi^{+} \pi^{-}$ analyses [283]. 

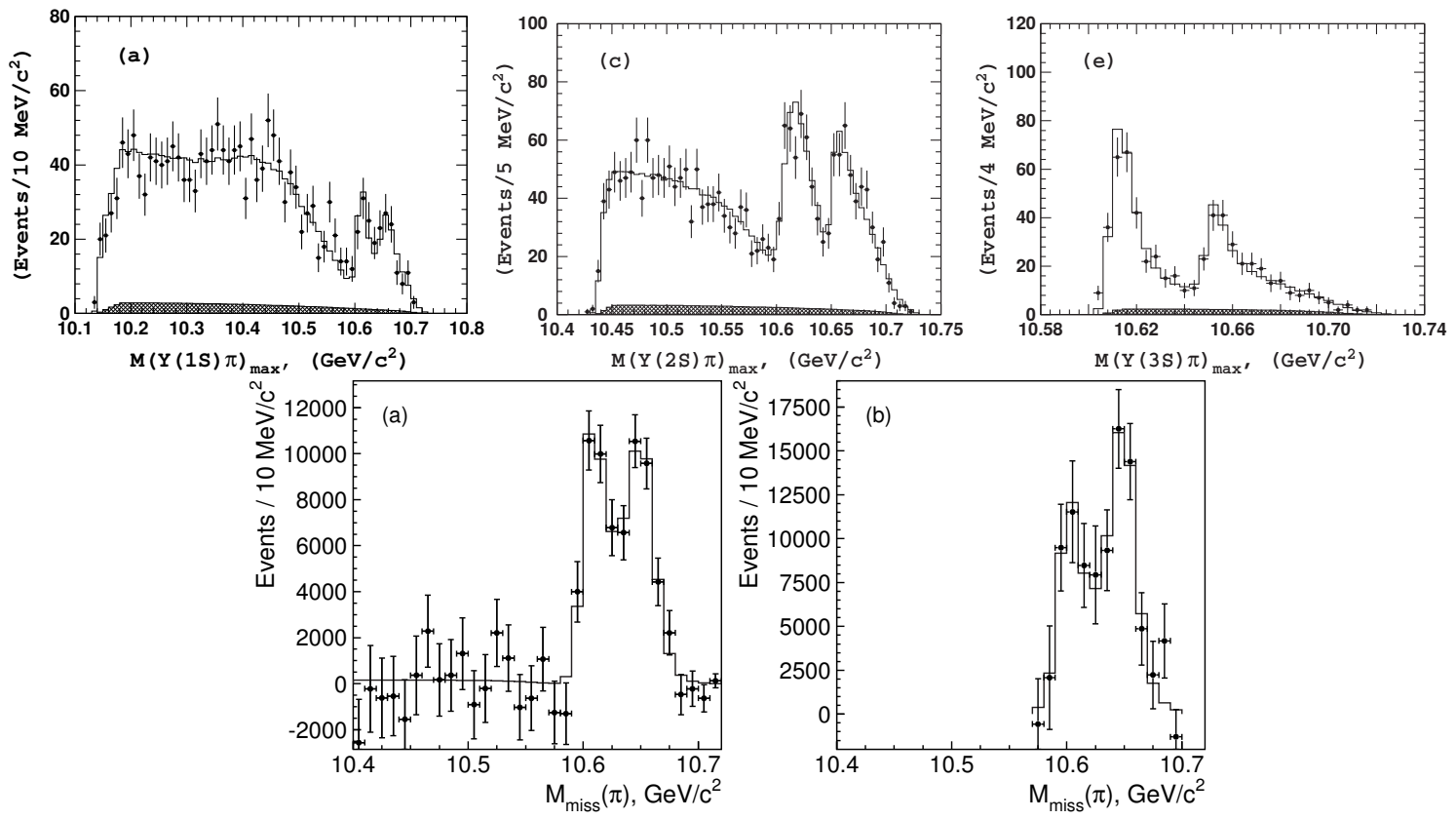

Figure 36: Comparison of fit results (open histogram) with experimental data (points with error bars) for events in the $\Upsilon(n S)$ (first 3 plots) and $h_{b}(n P)$ (last 2 plots) regions. From Belle [283].

The production of the $Z_{b}$ s saturates the transitions to $h_{b}(n P) \pi^{+} \pi^{-}$, which indicates for the $Z_{b}$ s to be superpositions of different heavy quark spin eigenstates. The analyses of the dipion angular distributions favor the $J^{P}=1^{+}$spin-parity assignment for both the states $[283,345]$. The decay modes constrain $C=-, G=+$.

Recently, Belle has been able to find the neutral isospin partner $Z_{b}(10610)^{0}$ [286] in $\Upsilon(5 S) \rightarrow \Upsilon(n S) \pi^{0} \pi^{0}$ decays. No significant signal of $Z_{b}^{\prime}(10650)^{0}$ is seen.

The proximity of these two peaks to the $B B^{*}$ and $B^{*} B^{*}$ thresholds stimulated the search of the $Z_{b}$ s in pairs of open bottom mesons [284, 285]. The Dalitz plots of $\Upsilon(5 S) \rightarrow\left(B B^{*}\right)^{-} \pi^{+}$and $\Upsilon(5 S) \rightarrow\left(B^{*} B^{*}\right)^{-} \pi^{+}$report a highly significant signal of $Z_{b}^{-}(10610) \rightarrow\left(B B^{*}\right)^{-}$and $Z_{b}^{-}(10650) \rightarrow\left(B^{*} B^{*}\right)^{-}$, respectively, with negligible continuum contribution. The $Z_{b}^{\prime-}(10650) \rightarrow\left(B B^{*}\right)^{-}$component, favored by phase space, is instead compatible with zero ${ }^{50}$. The plot of the invariant masses recoiling off the pion is shown in Figure 37. The best estimate for the branching ratios are reported in Table 14 , under the assumption the observed channels to saturate the resonance widths. This decay pattern is natural in the molecular picture, whereas Ali et al. had predicted the decay $Z_{b}^{\prime}(10650) \rightarrow \bar{B}^{*} B^{*}$ to be forbidden in the tetraquark model [346]. However, this conclusion was later overturned, considering that light spin flip can naturally occur in the decay [344]. A more detailed discussion on this subject is in Section 4.

Before the discovery of the $Z_{b} \mathrm{~s}$, the anomalously high rate of $\Upsilon(5 S)$ dipion transition lead to the speculation that two vector states were actually contributing: the actual $\Upsilon(5 S)$, and an exotic vector tetraquark state, the socalled $Y_{b}(10890)$ [347, 348]. A refit to the BABAR $R_{b}=\sigma\left(e^{+} e^{-} \rightarrow b \bar{b}\right) / \sigma\left(e^{+} e^{-} \rightarrow \mu^{+} \mu^{-}\right)$data [349] allowed for the presence of a narrow peak slightly heavier than the $\Upsilon(5 S)$. However the most recent analysis by Belle on the full dataset excluded the existence of such a narrow peak [350]. The same analysis shows a $2 \sigma$ discrepancy between the resonance parameters of the $\Upsilon(5 S)$ measured in $R_{b}$, and in $R_{\Upsilon \pi \pi}$. This also has suggested for the states seen in the two channels to be different [351]. Still, the Belle analysis comments that, while the $\Upsilon \pi \pi$ channel has basically no continuum contribution, the presence of many different open bottom threshold is expected to affect the lineshapes

\footnotetext{
${ }^{50} Z_{b}^{-}(10610) \rightarrow\left(B^{*} B^{*}\right)^{-}$is phase-space forbidden.
} 


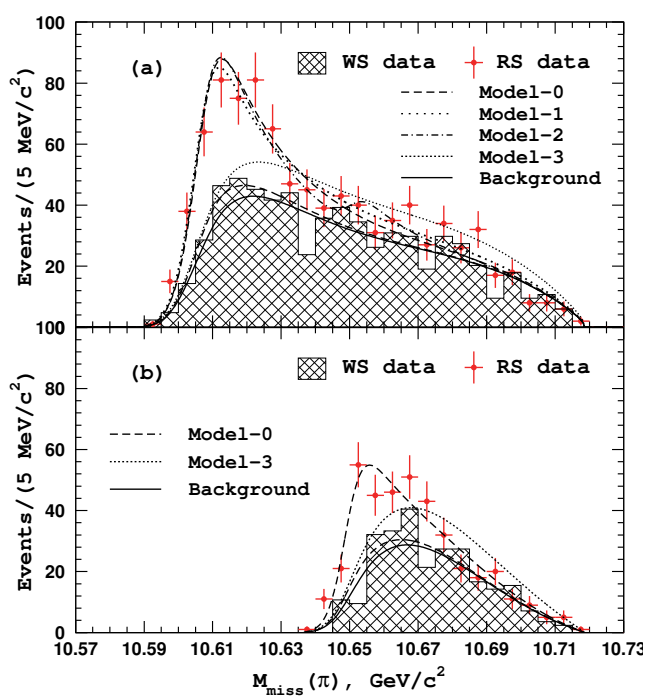

Figure 37: The $\Upsilon(5 S) \rightarrow \bar{B}^{(*)} B^{*} \pi$ channel measured by Belle [285]. The $Z_{b}(10610)$ and $Z_{b}^{\prime}(10650)$ dominate the $\bar{B} B^{*}$ and the $\bar{B}^{*} B^{*}$, respectively.

\begin{tabular}{lcc}
\hline \hline Channel & \multicolumn{2}{c}{ Fraction, \% } \\
& $Z_{b}(10610)$ & $Z_{b}^{\prime}(10650)$ \\
\hline$\Upsilon(1 \mathrm{~S}) \pi^{+}$ & $0.60 \pm 0.17 \pm 0.07$ & $0.17 \pm 0.06 \pm 0.02$ \\
$\Upsilon(2 \mathrm{~S}) \pi^{+}$ & $4.05 \pm 0.81 \pm 0.58$ & $1.38 \pm 0.45 \pm 0.21$ \\
$\Upsilon(3 \mathrm{~S}) \pi^{+}$ & $2.40 \pm 0.58 \pm 0.36$ & $1.62 \pm 0.50 \pm 0.24$ \\
$h_{b}(1 \mathrm{P}) \pi^{+}$ & $4.26 \pm 1.28 \pm 1.10$ & $9.23 \pm 2.88 \pm 2.28$ \\
$h_{b}(2 \mathrm{P}) \pi^{+}$ & $6.08 \pm 2.15 \pm 1.63$ & $17.0 \pm 3.74 \pm 4.1$ \\
$B^{+} \bar{B}^{* 0}+\bar{B}^{0} B^{*+}$ & $82.6 \pm 2.9 \pm 2.3$ & - \\
$B^{*+} \bar{B}^{* 0}$ & - & $70.6 \pm 4.9 \pm 4.4$ \\
\hline \hline
\end{tabular}

Table 14: List of branching fractions for the $Z_{b}^{+}(10610)$ and $Z_{b}^{+}(10650)$ decays. From Belle [285].

in $R_{b}$, leading to slightly different resonance parameters (see for example the model in [352]). Also, if no peaking background is included, the decay of the $\Upsilon(5 S)$ is saturated by the existing $Z_{b}^{(\prime)} \pi$ and $\Upsilon \pi \pi$ final states, and no room for $B_{s}^{(*)} \bar{B}_{s}^{(*)}$ is left, despite they represent the $20 \%$ of on-peak events. This inconsistency led Belle not to use $R_{b}$ data for the precise extraction of resonance parameters, thus resolving the discrepancy with $R_{\Upsilon \pi \pi}$. We conclude that, in the present condition, data do not support the existence of two different states close to the $\Upsilon(5 S)$. Instead, the possibility for the $\Upsilon(5 S)$ to contain a sizable tetraquark component is still viable.

The existence of the $Z_{b}$ s states immediately calls for a $X_{b}$, a hidden-bottom partner of the $X(3872)$, with $J^{P C}=1^{++}$. This is predicted by most of the models which fulfill heavy quark spin and flavor symmetry [344, 353], and it is generally expected to lay close to the $B B^{*}$ threshold. This state has been searched at Belle in the isospin-conserving $e^{+} e^{-} \rightarrow \gamma X_{b}(\rightarrow \Upsilon(1 S) \omega)$ channel at the $\Upsilon(5 S)$ peak [354], and at ATLAS in the isospin-violating inclusive $X_{b} \rightarrow$ $\Upsilon(1 S) \pi^{+} \pi^{-}$channel [355] ${ }^{51}$.

\footnotetext{
${ }^{51}$ This very channel has been searched in analogy to the $X(3872) \rightarrow J / \psi \pi^{+} \pi^{-}$decay. It is worth noticing that, if the isospin violation happens
} 


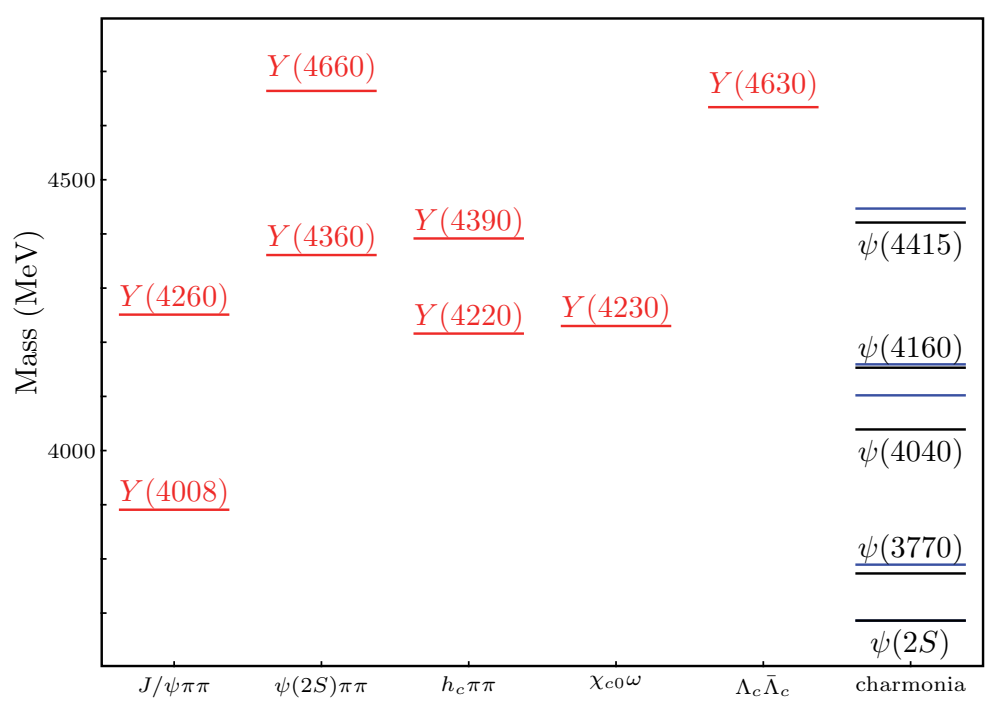

Figure 38: Exotic vector states divided by decay channel. In the right column, we report observed (black) and predicted (blue) charmonium levels Red line are exotic states.

\subsection{Vector $Y$ states}

The $e^{+} e^{-}$collider can directly produce states with unambiguous signature $J^{P C}=1^{--}$. This happens if the centerof-mass energy coincides with the position of a resonance, which requires the knowledge of the mass of the resonance itself ${ }^{52}$. Alternatively, a fraction of the events contains an additional energetic photon $\gamma_{\text {ISR }}$ emitted by the initial state, that effectively decreases the center-of-mass energy. One can thus scan a large range of $\sqrt{s}$ (which is function of the energy of the $\gamma_{\mathrm{ISR}}$ ) without varying the energy of the beams. The $\gamma_{\mathrm{ISR}}$ can be either detected (large angle ISR, good for exploring low $\sqrt{s}$ ), or lost in the beampipe (small angle ISR, good for exploring higher $\sqrt{s}$ ). In the latter case, the event is identified by requiring that the missing invariant mass is compatible with zero. This technique allowed the $B$-factories to search and discover many unexpected vectors, usually called $Y$ states. Their exotic assignment is mainly due to two reasons: $i$ ) the ordinary $\psi$ charmonia in that mass region are already well established, and there are no free slots for these new $Y \mathrm{~s}$, and $i$ ) they do not decay into open charm final states, which instead dominate the decay width of the ordinary $\psi$ above-threshold.

In Figure 38 we show the summary of the detected vectors, together with their main decay channel. The tetraquark picture is able to frame most of these states in a comprehensive picture - see Section 4.6 - identifying all the $Y$ states either as the lightest $L=1$ states, or as their radial excitations [8]. Other models provide different descriptions, on a case-by-case basis. For example, the $Y(4260)$ has been described in terms of a $\bar{D} D_{1}(2420)$ molecule, which would appear in the slightly displaced $Y(4220)$ and $Y(4230)$ peaks. However, it is important to stress that the resulting large binding energy of $\sim 70 \mathrm{MeV}$ pushes the state out of the range of applicability of the shallow states theory (Section 3). The $Y(4360)$ would be the $Y(4260)$ heavy quark spin partner, $\bar{D}^{*} D_{1}(2420)[55,357]$. The $Y(4660) / Y(4630)$ would be instead a $\psi(2 S) f_{0}(980)$ molecule [358]. Voloshin [147] proposed the hadrocharmonium model to describe the decay pattern of the $Y(4260)$ and $Y(4360)$ (see Section 7.1). Lattice QCD and constituent models usually identify a $c \bar{c} g$ hybrid vector state in the region of the $Y(4260)$ - see Section 7.2.

The first in the list is the $Y(4260)$, found at BABAR in 2005 decaying into $J / \psi \pi^{+} \pi^{-}$, and later confirmed by CLEO

at the hadron level, it will be sizable in the charm sector (since the difference in mass being $\left.M\left(D^{+} D^{*-}\right)-M\left(D^{0} \bar{D}^{* 0}\right) \sim 8 \mathrm{MeV}\right)$, but negligible in the bottom sector $\left(M\left(B^{+} B^{*-}\right) \simeq M\left(B^{0} \bar{B}^{* 0}\right)\right)$. However, if the isospin violation is driven by the heavy scale at a more fundamental level [80], it can occur in the $X_{b}$ as well.

${ }^{52}$ But fortune favors the bold [356]... 

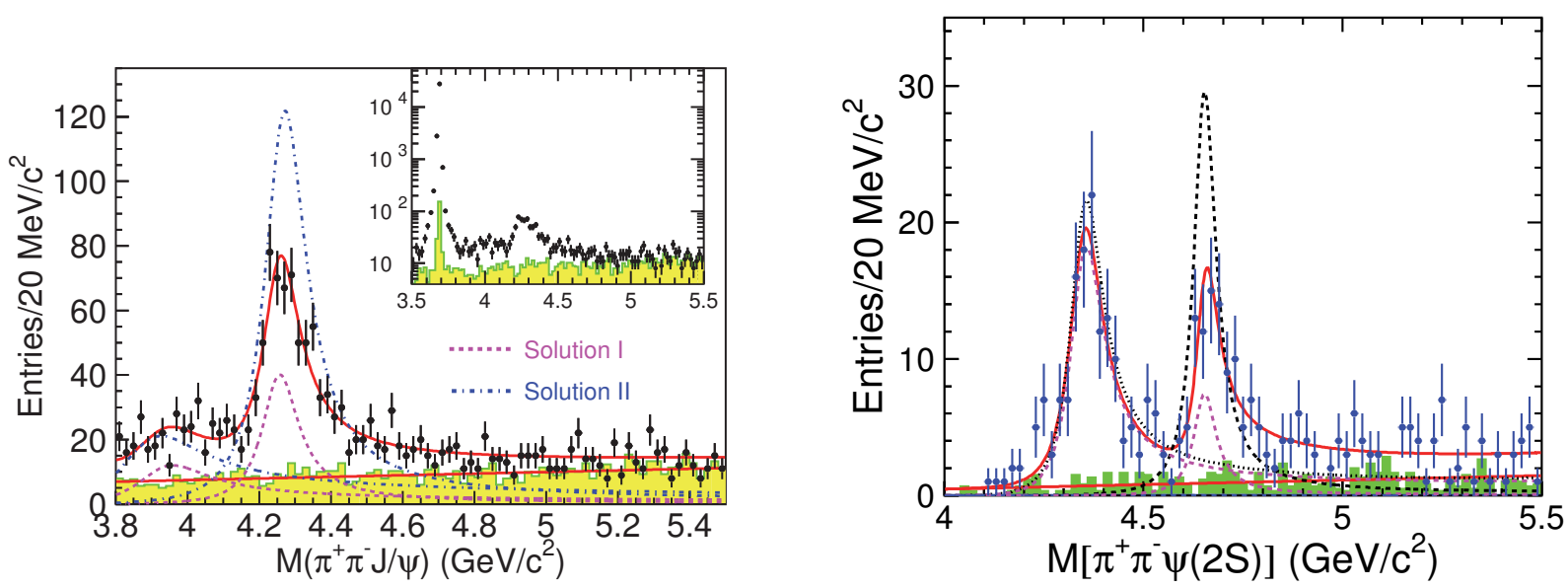

Figure 39: Belle analyses of $e^{+} e^{-} \rightarrow J / \psi \pi^{+} \pi^{-}$(left) [155] and $\rightarrow \psi(2 S) \pi^{+} \pi^{-}$(right) [267].

and Belle [155, 264, 270, 273, 276]. Evidence for $Y(4260) \rightarrow J / \psi \pi^{0} \pi^{0}$ was also reported by CLEO [272], whereas no clear sign of $Y(4260) \rightarrow J / \psi K^{+} K^{-}$or $\rightarrow K_{s}^{0} K_{S}^{0}$ has been seen so far [272, 359, 360]. The averaged values for mass and width are $M=(4251 \pm 9) \mathrm{MeV}$ and $\Gamma=(120 \pm 12) \mathrm{MeV}^{53}$. A thorough search of $Y(4260)$ in open charm final states has been performed by BABAR, Belle and CLEO- $c$, with no success. We report the list of most of the channels where the $Y(4260)$ has been searched, and the $90 \%$ upper limits, in Table 15. Instead, for the discovery mode, we have $\Gamma\left(Y(4260) \rightarrow e^{+} e^{-}\right) \times \mathcal{B}\left(Y(4260) \rightarrow J / \psi \pi^{+} \pi^{-}\right)=(9.2 \pm 1.0) \mathrm{eV}$. The electronic width can be constrained by looking at the inclusive $\sigma\left(e^{+} e^{-} \rightarrow\right.$ hadrons $)$, giving $\Gamma\left(Y(4260) \rightarrow e^{+} e^{-}\right)<580 \mathrm{eV}$ at $90 \%$ C.L. [361]. This estimate will be improved with the forthcoming data on the inclusive hadron cross section by BES III. This turns out to a dipion transition of $\Gamma\left(Y(4260) \rightarrow J / \psi \pi^{+} \pi^{-}\right) \sim 1 \mathrm{MeV}$, one order of magnitude larger than similar transitions of ordinary charmonia (and somehow similar to the dipion abundance in the $\Upsilon(5 S)$ transitions, see Section 8.3).

To investigate the nature of this resonance, it is important to understand whether the pion pair is dominated by any resonance. The updated $B A B A R$ analysis finds some evidence of $Y(4260) \rightarrow J / \psi f_{0}(980)$, although a relevant nonresonant component is still needed [271]. This might explain why the state does not decay into $\psi(2 S)^{54}$, since the decay $Y(4260) \rightarrow \psi(2 S) f_{0}(980)$ is phase-space forbidden (see also Section 3.4). In this case, a sizable $Y(4260) \rightarrow$ $J / \psi K \bar{K}$ is expected, which calls for a new analysis in this channel. For the transition to the exotic $Z_{c}$ s states, see Section 8.2 , but a proper energy scan is still due to understand whether the production of $Z_{c}$ s is dominated by the $Y(4260)$ or by continuum events.

The identification of the $Y(4260)$ as $\bar{D} D_{1}(2420)$ molecule calls for the search of the decay into these constituents. The analysis of the Dalitz plot $Y(4260) \rightarrow \bar{D} D^{*} \pi$ allows to search the intermediate narrow $D_{1}(2420)$ as a $D$-wave $D^{*} \pi$ resonance. BES III performed the analysis on both the single and double tagged samples, finding no $D_{1}$ component, but limited statistics prevented to draw strong conclusions [159, 251].

The radiative decays of the $Y(4260)$ can shed some light on the nature of this state. The production cross section $\left.\sigma\left(e^{+} e^{-} \rightarrow \gamma X(3872)\right) \times \mathcal{B}\left(X(3872) \rightarrow J / \psi \pi^{+} \pi^{-}\right)\right)$measured by BES III scales as a function of the center-of-mass energy consistently with a Breit-Wigner with $Y(4260)$ mass and width as parameters, consequently the observed events come from the intermediate resonant state and not from the continuum [110]. The estimate for the relative branching ratio is $\mathcal{B}(Y(4260) \rightarrow \gamma X(3872)) / \mathcal{B}\left(Y(4260) \rightarrow J / \psi \pi^{+} \pi^{-}\right) \sim 0.1^{55}$, which is relatively large. This radiative decay

\footnotetext{
${ }^{53}$ This average does not include the new results by BES III [108], which assume a different lineshape for the cross section, due to the interference of two different states. This is commented later on.

${ }^{54}$ The significance of a $Y(4260) \rightarrow \psi(2 S) \pi^{+} \pi^{-}$is $2.4 \sigma$ only [267].

55 assuming for the branching ratio $\mathcal{B}\left(X(3872) \rightarrow J / \psi \pi^{+} \pi^{-}\right)=5 \%$.
} 


\begin{tabular}{lll}
\hline \hline Final state & \multicolumn{1}{l}{ Upper limit (90\% C.L.) } & Experiment \\
\hline$\Gamma_{e e} \times \mathcal{B}(Y(4260) \rightarrow f)(\mathrm{eV})$ & \\
\hline$J / \psi K^{+} K^{-}$ & 1.2 & Belle [359] \\
$J / \psi \eta$ & 14.2 & Belle [362] \\
$\psi(2 S) \pi^{+} \pi^{-}$ & $\sim 12$ & Reanalysis [363] \\
$\phi \pi^{+} \pi^{-}$ & 0.4 & BABAR [364] \\
$K_{s}^{0} K^{+} \pi^{-}$ & 0.5 & BABAR [365] \\
$K^{+} K^{-} \pi^{0}$ & 0.6 & BABAR [365] \\
\hline $\mathcal{B}(Y(4260) \rightarrow f) / \mathcal{B}\left(Y(4260) \rightarrow J / \psi \pi^{+} \pi^{-}\right)$ \\
\hline$D \bar{D}$ & 1.0 & BABAR [366], \\
$D^{*} \bar{D}$ & 34 & BABAR [367] \\
$D^{*} \bar{D}^{*}$ & 11 & CLEO-c [368] \\
$D_{s}^{+} D_{s}^{-}$ & 0.7 & BABAR [369] \\
$D_{s}^{*+} D_{s}^{-}$ & 0.8 & CLEO-c [368] \\
$D_{s}^{*+} D_{s}^{*-}$ & 9.5 & CLEO-c [368] \\
$D^{0} D^{*-} \pi^{+}$ & 9 & Belle [370] \\
$D^{*} \bar{D} \pi$ & 15 & CLEO-c [368] \\
$D^{*} \bar{D}^{*} \pi$ & 8.2 & CLEO-c [368] \\
$h_{c} \pi^{+} \pi^{-}$ & $1.0^{\dagger}$ & CLEO [371] \\
$p \bar{p}$ & 0.13 & BABAR [372] \\
\hline$\sigma\left(e^{+} e^{-} \rightarrow f\right)(\mathrm{pb})$ & \\
\hline$\chi_{c 1} \omega$ & $18(\sqrt{s}=4.31 \mathrm{GeV})$ & BES III [269] \\
$\chi_{c 2} \omega$ & $11(\sqrt{s}=4.36 \mathrm{GeV})$ & BES III [269] \\
\hline \hline
\end{tabular}

Table 15: Upper limits for $Y(4260)$ into different final states. ${ }^{\dagger}$ This has to be updated with new BES III results [108, 109].

is naturally expected in the tetraquark model as an E1 transition of the orbitally excited tetraquark to its ground state, with no spin change [8]. These predictions, as well as the ones under the molecular [56] and hybrid hypotheses [190], can be tested by more precise measurements of these branching ratios.

In the same $J / \psi \pi^{+} \pi^{-}$final state, Belle reported also a broad structure named $Y(4008)$ [155, 264], with $M=$ $(3890.8 \pm 40.5 \pm 11.5) \mathrm{MeV}$ and $\Gamma=(254.5 \pm 39.5 \pm 13.6) \mathrm{MeV}$. This is at odds with $B A B A R$ [271], which does not observe any structure in that region. Very recently, BES III published the results of a precise scan analysis [108] (see Figure 40), which finds a much broader state, $M=\left(3812.6_{-96.6}^{+61.9}\right) \mathrm{MeV}$ and $\Gamma=\left(476.9_{-64.8}^{+78.4}\right) \mathrm{MeV}$. Alternatively, it is substituted by an exponential background, obtaining an equally good fit. In the same analysis, the asymmetric shape of the $Y(4260)$ turns out to be due to the interference of a narrower $Y(4260)$, with mass and width $M=(4222.0 \pm 3.1 \pm 1.4) \mathrm{MeV}$ and $\Gamma=$ $(44.1 \pm 4.3 \pm 2.0) \mathrm{MeV}$, with a slightly heavier one, with $M=(4320.0 \pm 10.4 \pm 7.0) \mathrm{MeV}$ and $\Gamma=\left(101.4_{-19.7}^{+25.3} \pm 10.2\right) \mathrm{MeV}$. The latter is compatible with the state observed in $e^{+} e^{-} \rightarrow \psi(2 S) \pi \pi$ discussed below. More statistics will help in solving the controversy about the $Y(4008)$.

The analysis of the $\psi(2 S) \pi^{+} \pi^{-}$channel gave some surprises: instead of finding the $Y(4260), B A B A R$ and Belle 

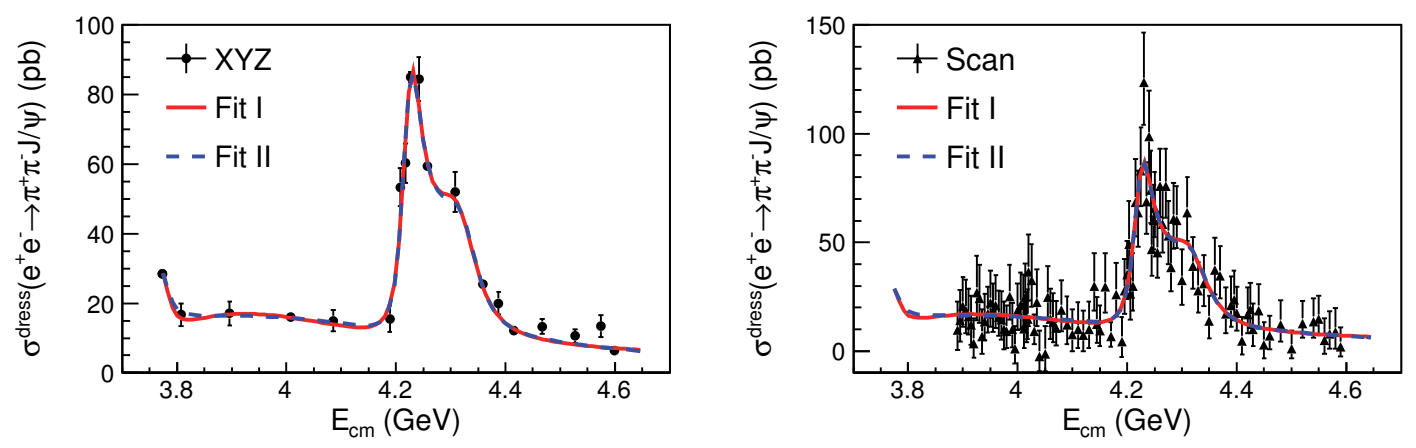

Figure 40: Measured dressed cross section $\sigma^{\mathrm{dress}}\left(e^{+} e^{-} \rightarrow \pi^{+} \pi^{-} J / \psi\right.$ at BES III [108], and simultaneous fit to the high-statistics datasets (left) and to the energy scan data (right) with the coherent sum of the Breit-Wigner functions for the $Y(4008)$, the $Y(4260)$, and the $Y(4360)$ (red solid curves), or substituting the $Y(4008)$ with a coherent exponential continuum.

observed other two resonances: the $Y(4360)$ and the $Y(4660)[267,275,276,373]$, with masses and widths $M=$ $(4346.2 \pm 6.3) \mathrm{MeV}, \Gamma=(102 \pm 10) \mathrm{MeV}$, and $M=(4657 \pm 11) \mathrm{MeV}, \Gamma=(70 \pm 11 \pm 3) \mathrm{MeV}$, respectively. The latter has not been seen in $J / \psi \pi \pi$, despite the larger phase space. The decay $Y(4360) \rightarrow J / \psi \pi^{+} \pi^{-}$has been recently observed by BES III [108], which is at odds with the combined analysis of BABAR and Belle data [81]. In Figure 39 we report some distributions of $J / \psi \pi^{+} \pi^{-}$and $\psi(2 S) \pi^{+} \pi^{-}$by Belle.

The preference of tetraquark states to decay into baryons was understood since the origins of the model [7, 78, 79, 82]. Motivated by this, Belle searched for vector resonances decaying into $\Lambda_{c}^{+} \Lambda_{c}^{-}$[281]. A structure (the $Y(4630)$ ) has actually been found near the baryon threshold, with Breit-Wigner parameters $M=\left(4634_{-7-8}^{+8+5}\right) \mathrm{MeV}$ and $\Gamma=$ $\left(92_{-24-21}^{+40+10}\right) \mathrm{MeV}$. A combined fit of the $\psi(2 S) \pi^{+} \pi^{-}$and $\Lambda_{c}^{+} \Lambda_{c}^{-}$spectra concluded that the two structures $Y(4630)$ and $Y(4660)$ can indeed be the same state, with a strong preference for the baryonic decay mode: $\mathcal{B}(Y(4660) \rightarrow$ $\left.\Lambda_{c}^{+} \Lambda_{c}^{-}\right) / \mathcal{B}\left(Y(4660) \rightarrow \psi(2 S) \pi^{+} \pi^{-}\right)=25 \pm 7$ [81]. The possibility these two structures to be actually distinct has been explored in [8].

BES III also measured the $e^{+} e^{-} \rightarrow h_{c} \pi^{+} \pi^{-}$cross sections at center-of-mass energies varying from 3.896 to $4.600 \mathrm{GeV}[109,161]$. The values of the cross sections are similar to the $e^{+} e^{-} \rightarrow J / \psi \pi^{+} \pi^{-}$one, but the lineshape looks different (see Figure 41). The $h_{c} \pi^{+} \pi^{-}$spectrum has been fitted first by Yuan [112, 113], which found a significant signal for a $Y(4220)$, while the higher mass region could be described either by a $Y(4290)$ state, or by a phase-space background. The very recent energy scan by BES III [109] has confirmed the former hypothesis, giving for the two resonances masses and widths $M=(4218.4 \pm 4.0 \pm 0.9) \mathrm{MeV}$ and $\Gamma=(66.0 \pm 9.0 \pm 0.4) \mathrm{MeV}$, $M=(4391.6 \pm 6.3 \pm 1.0) \mathrm{MeV}$ and $\Gamma=(139.5 \pm 16.1 \pm 0.6) \mathrm{MeV}$. A somewhat similar signal has been seen by BES III in $e^{+} e^{-} \rightarrow \chi_{c 0} \omega[269,374]$ at a mass of $M=(4230 \pm 8) \mathrm{MeV}$ and a width of $\Gamma=(38 \pm 12) \mathrm{MeV}$, again not compatible with $Y(4260)$ parameters. The hypothesis that the $Y(4220)$ and $Y(4230)$ are indeed the same tetraquark state has been studied in [111]. The possibility that, instead, the state in $\chi_{c 0} \omega$ is the ordinary $\psi(4160)$ has been proposed in [375]. New BES III data suggest for this $Y(4220)$ to coincide with the narrow one observed in $J / \psi \pi^{+} \pi^{-}$. It is clear that a combined refit of all available data in this sector is needed to establish the existence of many of the unsettled $Y$ states.

\subsection{The $Z(4430)$}

The childbirth of the Z(4430) was long and troubled. The state was claimed in 2007 by Belle, as a peak in the $B \rightarrow K\left(\psi(2 S) \pi^{+}\right)$channel [376], and it was the first observation of a charged charmoniumlike state. Such analyses can suffer from the rich structure of $K \pi$ resonances, which reflect into the $\psi(2 S) \pi$ channel and might create fake peaks. However, Belle considered that the events with $M\left(\psi(2 S) \pi^{-}\right) \sim 4430 \mathrm{MeV}$ correspond to events with $\cos \theta_{K \pi} \simeq 0.25$, i.e. an angular region where interfering $L=0,1,2$ partial waves cannot produce a single peak without creating other 

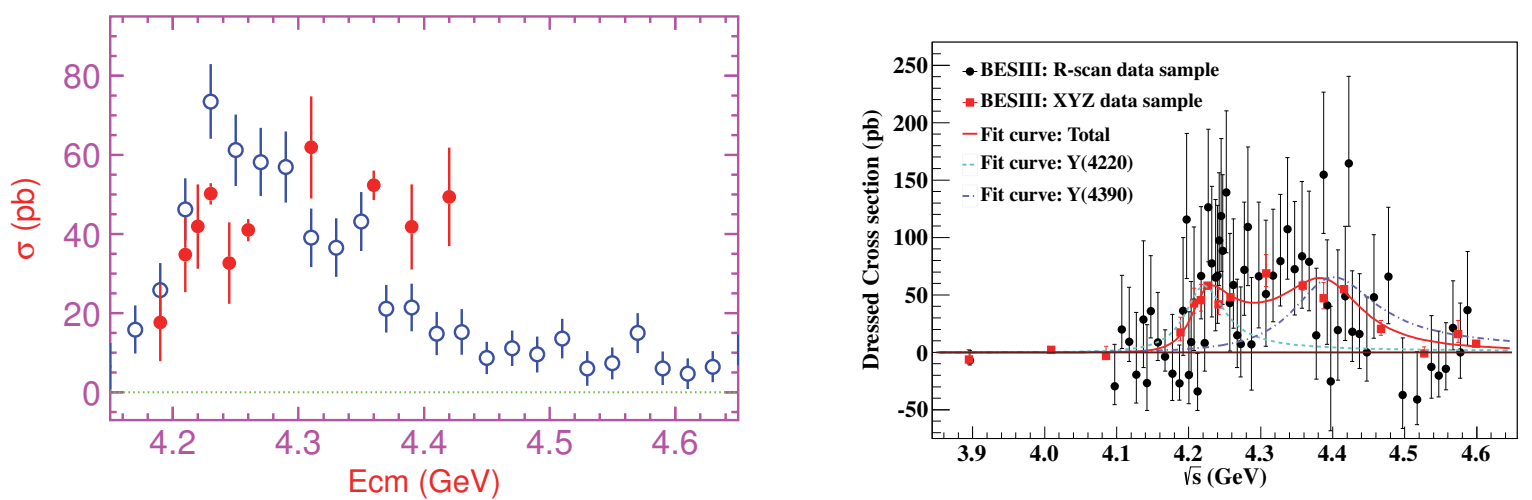

Figure 41: Left panel: BES III data of $e^{+} e^{-} \rightarrow h_{c} \pi^{+} \pi^{-}$(red dots) [161] compared to Belle data of $e^{+} e^{-} \rightarrow J / \psi \pi^{+} \pi^{-}$(blue circles) [155]. From Yuan [112, 113]. Right panel: new BES III data of $e^{+} e^{-} \rightarrow h_{c} \pi^{+} \pi^{-}$[109]. The dash (dash-dot) curve shows the contribution from the two structures $Y(4220)(Y(4390))$

larger structures elsewhere. The subsequent $B A B A R$ analysis parametrized the $K \pi$ spectrum with a model-independent technique, i.e. just projecting the $K \pi$ spectrum over partial waves without assuming any specific $K^{*}$ content. The reflection of this system could fit the $\psi(2 S) \pi$ spectrum without needing any additional $Z(4430)$ resonance. The final dataset Belle's Dalitz analysis confirmed the presence of a resonance [277, 278]. Finally, LHCb put beyond doubt the existence of this state, first with a high-statistics 4D fit to the usual isobar model, and later applying the BABAR's model-independent analysis [280, 377]. This state is extremely interesting, because is far from any reasonable opencharm threshold with the correct quantum numbers ${ }^{56}$. The averaged mass and width are $M=(4478 \pm 17) \mathrm{MeV}$ and $\Gamma=(180 \pm 31) \mathrm{MeV}$, whereas the favored signature is $J^{P C}=1^{+-}$. The LHCb analysis also stresses the resonant nature of the $Z(4430)$, by fitting six independent complex numbers in bins of $M^{2}(\psi(2 S) \pi)$, instead of forcing the Breit-Wigner lineshape. The result is consistent with the Breit-Wigner expectation (see Figure 42), although other mechanisms can mimic a similar resonant behavior — see Section 7.3.
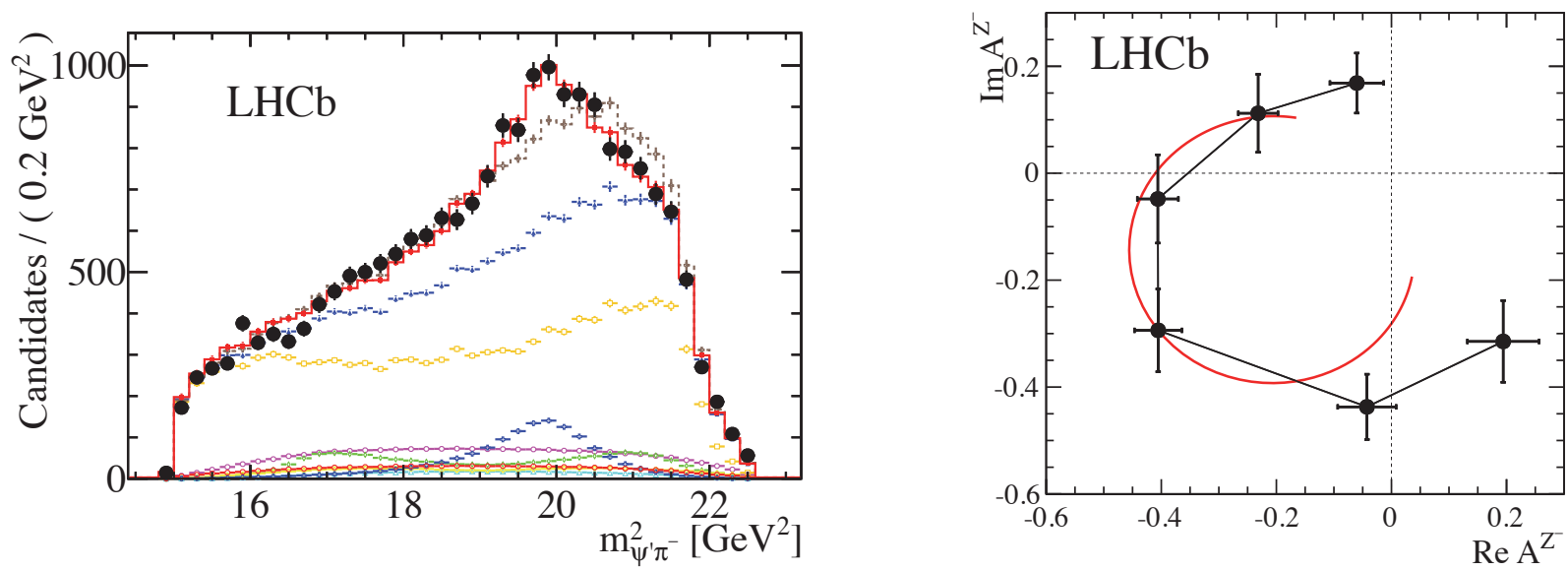

Figure 42: Invariant mass distributions in $\psi(2 S) \pi^{-}$channel (left) and resonant behavior (right) according to LHCb [280]. In the left panel, the red solid (brown dashed) curve shows the fit with (without) the additional Z(4430) resonance. In the right panel, the complex value of the $Z$ (4430) fitted amplitude for six bins of $M(\psi(2 S) \pi)$ is shown. The red curve is the prediction from the Breit-Wigner formula with a resonance mass (width) of 4475 (172) MeV.

\footnotetext{
${ }^{56}$ unless one resorts to the radial excitations $D(2 S)$ and $D^{*}(2 S)$, but their broad width of $\sim 100 \mathrm{MeV}$ challenges any proper definition of threshold.
} 

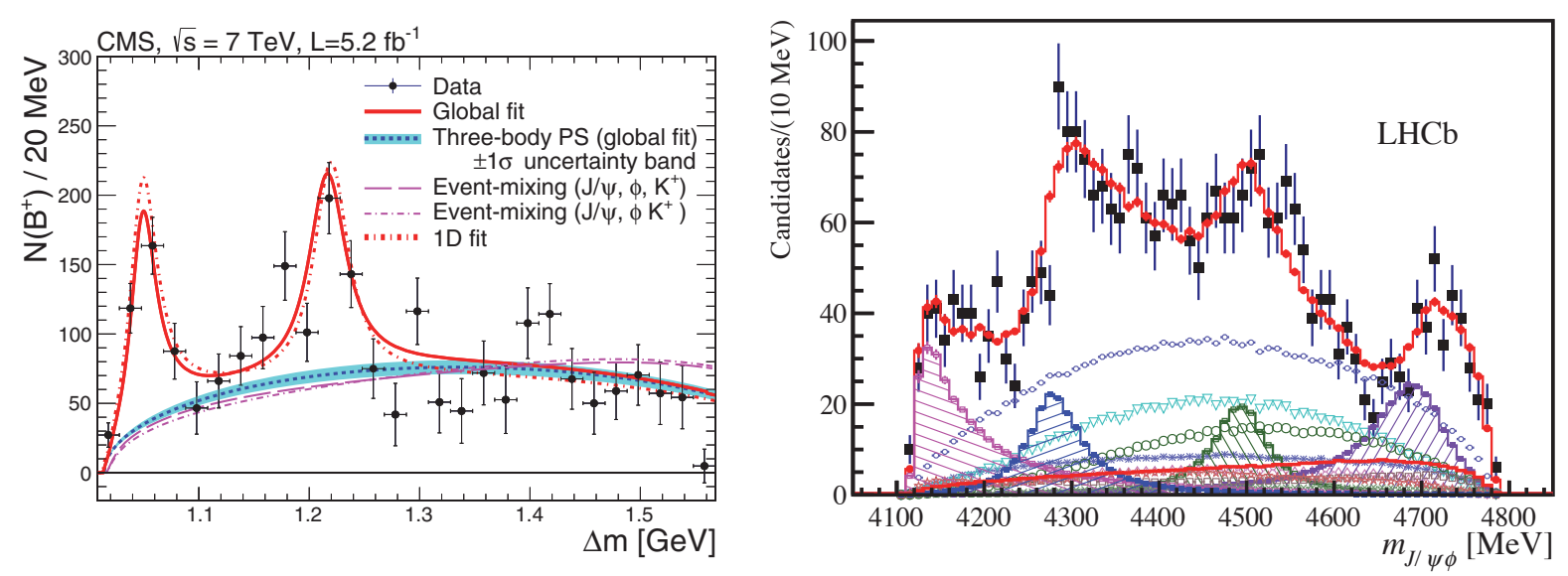

Figure 43: Invariant mass distributions of $J / \psi \phi$, from the $B^{+} \rightarrow K^{+} J / \psi \phi$ data by CMS ([134], left panel) and LHCb ([135, 136], right panel).

Belle also searched for the same state in the $B \rightarrow K\left(J / \psi \pi^{+}\right)$channel [268]. A $4 \sigma$ signal consistent with the $Z(4430)$ is found. We can thus compare

$$
\mathcal{B}\left(\bar{B}^{0} \rightarrow K^{-} Z(4430)^{+}\right) \times \mathcal{B}\left(Z(4430)^{+} \rightarrow \psi(2 S) \pi^{+}\right)=\left(6.0_{-2.0-1.4}^{+1.7+2.5}\right) \times 10^{-5} .
$$

with

$$
\mathcal{B}\left(\bar{B}^{0} \rightarrow K^{-} Z(4430)^{+}\right) \times \mathcal{B}\left(Z(4430)^{+} \rightarrow J / \psi \pi^{+}\right)=\left(5.4_{-1.0-0.6}^{+4.0+1.1}\right) \times 10^{-6},
$$

i.e. the decay into $\psi(2 S)$ is larger by one order of magnitude, despite the smaller phase space.

\subsection{The $[c s][\bar{c} \bar{s}]$ sector}

The CDF experiment announced a resonance close to threshold in $J / \psi \phi$ invariant mass, in the channel $B^{+} \rightarrow$ $J / \psi \phi K^{+}[378,379]$. Since the creation of a $s \bar{s}$ pair is OZI suppressed, the very existence of such states likely requires exotic interpretations. This state was called $X(4140)$, or sometimes $Y(4140)$.

The first low-statistics analysis by LHCb [381], and the analyses by Belle and BABAR [380, 383] were not able to see any significant signal in this channel. Belle searched this state in $\gamma \gamma$ fusion, driven by a molecular prediction [385], but found no $X(4140)$ [274]. Similarly, BES III saw no $X(4140)$ signal in the $e^{+} e^{-} \rightarrow \gamma(J / \psi \phi)$ process [386]. Instead, $\mathrm{D} \varnothing$ [382] and CMS [134] have confirmed the observation, with significances of $\sim 3 \sigma$ and $>5 \sigma$, respectively, although with slightly inconsistent values for the mass and width. $\mathrm{D} \varnothing$ found the state also in prompt production [384]. CDF, CMS and $\mathrm{D} \varnothing$ also reported evidence for a second peak at a mass of $M \sim 4300 \mathrm{MeV}$ and $\Gamma \sim 35 \mathrm{MeV}$.

Very recently, LHCb published the preliminary amplitude analysis of the full 6D Dalitz distributions [135, 136]. The results are impressive: they not only confirm both the $X(4140)$ and the second peak, although with much larger widths, but they also find other two heavier states. The situation is resumed in Table 16. The two lighter states, $X(4140)$ and $X(4274)$, have $J^{P C}=1^{++}$, and masses and widths $M=\left(4146.5 \pm 4.5_{-2.8}^{+4.6}\right) \mathrm{MeV}, \Gamma=\left(83 \pm 21_{-14}^{+21}\right) \mathrm{MeV}$, and $M=\left(4273.3 \pm 8.3_{-3.6}^{+17.2}\right) \mathrm{MeV}, \Gamma=\left(56 \pm 11_{-11}^{+8}\right) \mathrm{MeV}$, respectively. The two heavier ones, $X(4500)$ and $X(4700)$, are scalars $J^{P C}=0^{++}$, and have resonance parameters $M=\left(4506 \pm 11_{-15}^{+12}\right) \mathrm{MeV}, \Gamma=\left(92 \pm 21_{-20}^{+21}\right) \mathrm{MeV}$, and $M=$ $\left(4704 \pm 10_{-24}^{+14}\right) \mathrm{MeV}, \Gamma=\left(120 \pm 31_{-33}^{+42}\right)$, respectively.

In Figure 43 we show the $M(J / \psi \phi)$ distribution measured at CMS and LHCb. The discrepancy between the new $\mathrm{LHCb}$ results and all the previous ones deserves some comments. The excellent particle ID, and the large statistics 


\begin{tabular}{|c|c|c|c|c|c|}
\hline Experiment & Events & Mass (MeV) & Width $(\mathrm{MeV})$ & Sign. & Fraction $\%$ \\
\hline \multicolumn{6}{|c|}{$X(4140)$ peak } \\
\hline $\mathrm{CDF}(2008), 2.7 \mathrm{fb}^{-1}[378]$ & $58 \pm 10$ & $4143.0 \pm 2.9 \pm 1.2$ & $11.7_{-5.0}^{+8.3} \pm 3.7$ & $3.8 \sigma$ & \\
\hline Belle (2009) [380] & $325 \pm 21$ & 4143.0 fixed & 11 fixed & $1.9 \sigma$ & \\
\hline $\operatorname{CDF}(2011) 6.0 \mathrm{fb}^{-1}[379]$ & $115 \pm 12$ & $4143.4_{-3.0}^{+2.9} \pm 0.6$ & $15.3_{-6.1}^{+10.4} \pm 2.5$ & $5.0 \sigma$ & $14.9 \pm 3.9 \pm 2.4$ \\
\hline $\operatorname{LHCb}(2011) 0.37 \mathrm{fb}^{-1}[381]$ & $346 \pm 20$ & 4143.4 fixed & 15.3 fixed & $1.4 \sigma$ & $<7 @ 90 \%$ C.L. \\
\hline CMS (2013) $5.2 \mathrm{fb}^{-1}[134]$ & $2480 \pm 160$ & $4148.0 \pm 2.4 \pm 6.3$ & $28_{-11}^{+15} \pm 19$ & $5.0 \sigma$ & $10 \pm 3$ (stat.) \\
\hline $\mathrm{D} \varnothing(2013) 10.4 \mathrm{fb}^{-1}[382]$ & $215 \pm 37$ & $4159.0 \pm 4.3 \pm 6.6$ & $19.9 \pm 12.6_{-8.0}^{+1.0}$ & $3.1 \sigma$ & $21 \pm 8 \pm 4$ \\
\hline BABAR (2014) [383] & $189 \pm 14$ & 4143.4 fixed & 15.3 fixed & $1.6 \sigma$ & $<13.3 @ 90 \%$ C.L. \\
\hline \multicolumn{6}{|l|}{$\mathrm{D} \varnothing(2015) 10.4 \mathrm{fb}^{-1}[384]$} \\
\hline \multicolumn{2}{|l|}{$p \bar{p} \rightarrow J / \psi \phi+$ All (prompt) } & \multirow{2}{*}{$4152.5 \pm 1.7_{-5.4}^{+6.2}$} & \multirow{2}{*}{$16.3 \pm 5.6 \pm 11.4$} & $4.7 \sigma$ & \\
\hline \multicolumn{2}{|l|}{$p \bar{p} \rightarrow J / \psi \phi+$ All (non-prompt) } & & & $5.7 \sigma$ & \\
\hline Average & & $4145.5 \pm 2.3$ & $14.9 \pm 4.4$ & & \\
\hline \multicolumn{6}{|c|}{ Higher peaks } \\
\hline CDF $6.0 \mathrm{fb}^{-1}[379]$ & $115 \pm 12$ & $4274.4_{-6.7}^{+8.4} \pm 1.9$ & $32.3_{-15.3}^{+21.9} \pm 7.6$ & $3.1 \sigma$ & \\
\hline LHCb $0.37 \mathrm{fb}^{-1}[381]$ & $346 \pm 20$ & 4274.4 fixed & 32.3 fixed & & $<8$ @ 90\%C.L. \\
\hline CMS $5.2 \mathrm{fb}^{-1}[134]$ & $2480 \pm 160$ & $4313.8 \pm 5.3 \pm 7.3$ & $38_{-15}^{+30} \pm 16$ & & \\
\hline $\mathrm{D} \varnothing 10.4 \mathrm{fb}^{-1}[382]$ & $215 \pm 37$ & $4328.5 \pm 12.0$ & 30 fixed & & \\
\hline BABAR [383] & $189 \pm 14$ & 4274.4 fixed & 32.3 fixed & $1.2 \sigma$ & $<18.1$ @ 90\% C.L. \\
\hline Belle [274] & $\gamma \gamma \rightarrow J / \psi \phi$ & $4350.6_{-5.1}^{+4.6} \pm 0.7$ & $13_{-9}^{+18} \pm 4$ & $3.2 \sigma$ & \\
\hline \multicolumn{6}{|c|}{ New LHCb results $[135,136]$} \\
\hline$X(4140), J^{P C}=1^{++}$ & $4289 \pm 151$ & $4146.5 \pm 4.5_{-2.8}^{+4.6}$ & $83 \pm 21_{-14}^{+21}$ & $8.4 \sigma$ & $13 \pm 3.2_{-2.0}^{+4.8}$ \\
\hline$X(4274), J^{P C}=1^{++}$ & $4289 \pm 151$ & $4273.3 \pm 8.3_{-3.6}^{+17.2}$ & $56 \pm 11_{-11}^{+8}$ & $6.0 \sigma$ & $7.1 \pm 2.5_{-2.4}^{+3.5}$ \\
\hline$X(4500), J^{P C}=0^{++}$ & $4289 \pm 151$ & $4506 \pm 11_{-15}^{+12}$ & $92 \pm 21_{-20}^{+21}$ & $6.1 \sigma$ & $6.6 \pm 2.4_{-2.3}^{+3.5}$ \\
\hline$X(4700), J^{P C}=0^{++}$ & $4289 \pm 151$ & $4704 \pm 10_{-24}^{+14}$ & $120 \pm 31_{-33}^{+42}$ & $5.6 \sigma$ & $12 \pm 5_{-5}^{+9}$ \\
\hline
\end{tabular}

Table 16: Results related to the $J / \psi \phi$ resonances. We show the results of the previous experiments, with measured masses, widths and fit fractions, and compare with latest $\mathrm{LHCb}$ results. In the second column, we report the total number of $B^{+} \rightarrow J / \psi \phi K^{+}$events. 
allowed LHCb to have a very precise sample with low background, and to perform the full 6D fit. The fit included up to $5 \phi K^{+}$Breit-Wigners, consistent with known or predicted $K^{*}$ resonances. Still, a sizable nonresonant component is needed for a good fit. All these are summed coherently to the four $J / \psi \phi$ Breit-Wigners. On the other hand, the other analyses just fitted the 1D $J / \psi \phi$ projection because of limited statistics, adding incoherently one or two Breit-Wigners to a phase-space background. The incoherent background in general tends to produce more narrow widths than the (the same happened for the $Z(4430)$, Section 8.5), and could justify the mismatch. However, the $X(4140)$ is observed by $\mathrm{D} \varnothing$ in inclusive production [384]. In this case, the coherent sum of signal and background is meaningless, and the incoherent sum gives a width of $\Gamma=16.3 \pm 5.6 \pm 11.4$, much narrower than the one observed at $\mathrm{LHCb}$, and compatible with the previous observation. An independent observation of this state in inclusive production might help resolving this controversy.

\subsection{Pentaquark states}

In July 2015, LHCb reported the observation of two peaks, denoted $P_{c}(4380)^{+}$and $P_{c}(4450)^{+}$, in the invariant mass of $J / \psi p$ in the $\Lambda_{b}^{0} \rightarrow J / \psi p K^{-}$decay [114]. In the Dalitz plot in Figure 44 a band in the $J / \psi p$ is indeed visible, and clearly extends beyond the $\Lambda^{*}$ region $\left(m_{K p}^{2}<4 \mathrm{GeV}^{2}\right)$. The full amplitude fit cannot satisfactorily describe the data without including these two Breit-Wigner shaped resonances (Figure 45). The lighter state has a mass $M=$ $4380 \pm 8 \pm 29 \mathrm{MeV}$ and a width $\Gamma=205 \pm 18 \pm 86 \mathrm{MeV}$, while the heavier one has a mass $M=4449.8 \pm 1.7 \pm 2.5 \mathrm{MeV}$ and a width $\Gamma=39 \pm 5 \pm 19 \mathrm{MeV}$. The interference pattern of the two resonances in different bins of the helicity angle require them to have opposite parities, and the preferred $J^{P}$ are $\left(\frac{3}{2}^{-}, \frac{5}{2}^{+}\right)$, although $\left(\frac{3}{2}^{+}, \frac{5}{2}^{-}\right)$and $\left(\frac{5}{2}^{+}, \frac{3}{2}^{-}\right)$are not excluded. The higher mass state has a fit fraction of $(4.1 \pm 0.5 \pm 1.1) \%$, and the lower mass state of $(8.4 \pm 0.7 \pm 4.2) \%$, of the total sample. To study the resonant behavior of the two states, the amplitudes are represented as the combination of independent complex numbers at six equidistant points in the range $\pm \Gamma_{0}=39 \mathrm{MeV}$ around the as determined in the default fit. Real and imaginary parts of the amplitude are interpolated in mass between the fitted points. The resulting Argand diagram, shown in Figure 46, is consistent with a rapid motion of the $P_{c}(4450)^{+}$phase when its magnitude reaches the maximum, whereas no strong conclusion can be drawn for the wider $P_{c}(4380)^{+}$.

A new analysis by $\mathrm{LHCb}$ has recently confirmed the existence of pentaquark-like structure with the same modelindependent used in the analysis of the $Z(4430)$ (see Section 8.5), i.e. without assuming any Breit-Wigner lineshape for the $\Lambda^{*}$, but just projecting the $M(K p)$ invariant mass in partial waves, and studying the reflection in $M(J / \psi p)$. If no pentaquark is included, the $M(J / \psi p)$ distribution cannot reproduce data [387]. Finally, LHCb studied also the Cabibbo-suppressed $\Lambda_{b}^{0} \rightarrow J / \psi p \pi^{+}$channel. Also in this case, the $N^{*}$ reflections are not able to reproduce the $J / \psi \pi^{+}$spectrum, but lack of statistics prevents the resolving of different structures (possibly $P_{c}(4380), P_{c}(4450)$, or $Z(4200))$ [388].

A diquark-diquark-quark description has been proposed in [117, 389, 390] (see also Section 4.7). The molecular models can describe the narrow state, either as a $\bar{D}^{(*)} \Sigma_{c}$ molecule [391], or as a $\chi_{c 1} p$ molecule [218], but cannot at the same time describe the broader state, nor with opposite parity to the narrow one. To confirm the LHCb discovery, a search in another channel is needed. For example, Refs. [160, 392, 393] have proposed to search the pentaquark in photoproduction, estimating sizable cross sections. 


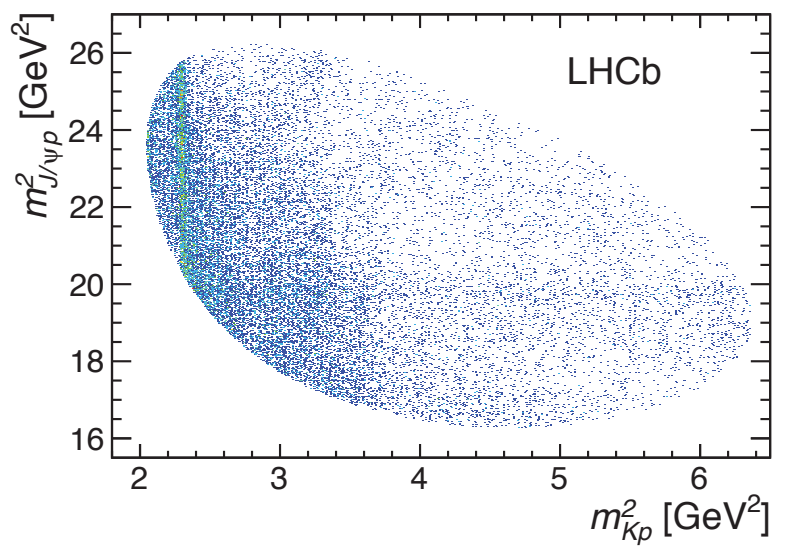

Figure 44: Invariant mass squared of $K p$ versus $J / \psi p$ for candidates within $\pm 15 \mathrm{MeV}$ of the $\Lambda_{b}^{0}$ mass [114].
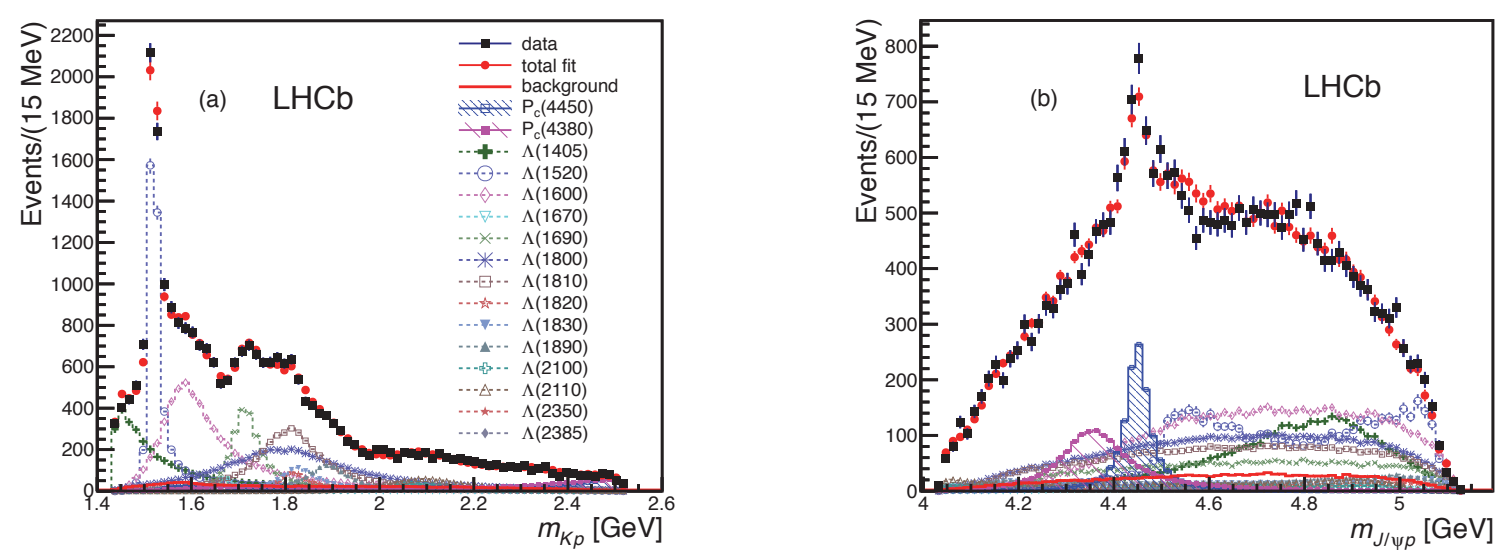

Figure 45: Results for the full amplitude fit in the $m_{K p}$ (left) and $m_{J / \psi} p$ variables. The data are shown as (black) squares with error bars, while the (red) circles show the results of the fit. The error bars on the points showing the fit results are due to simulation statistics [114]. 


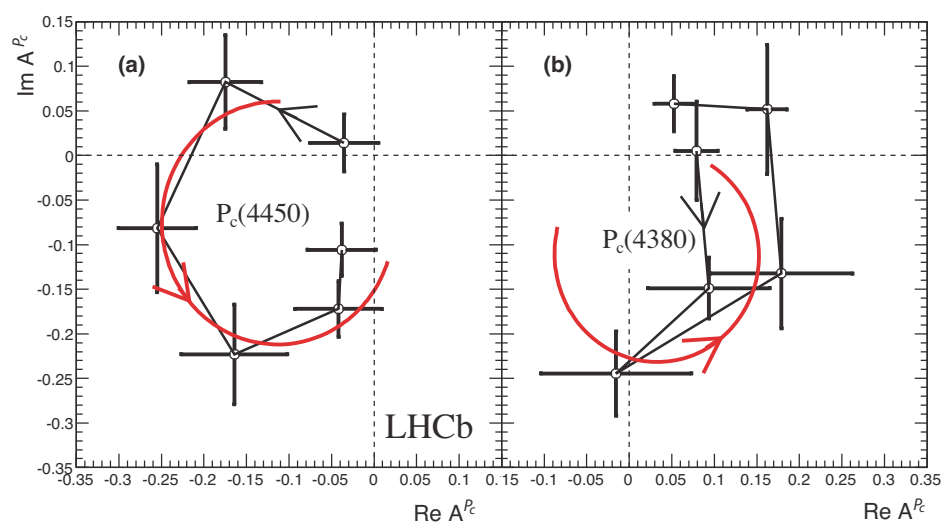

Figure 46: Fitted values of the real and imaginary parts of the amplitudes for the favored spin fit for the $P_{c}(4450)^{+}$state (left) and for the $P_{c}(4380)^{+}$ state (right), each divided into six $m_{J / \psi}$ bins of equal width shown in the Argand diagrams as connected points with error bars $\left(m_{J / \psi} p\right.$ increases counterclockwise). The solid (red) curves are the predictions from the Breit-Wigner formula [114].

\subsection{Other states}

$\chi_{c \mathbf{0}} \mathbf{( 3 9 1 5 )}$ - A resonance decaying into $J / \psi \omega$ was observed by Belle and BABAR in the $B \rightarrow K(J / \psi \omega)$ channel [257-259]. Both experiments found the same state in $\gamma \gamma$ fusion [260, 261]. The latter production mechanism constrains $C=+, J \neq 1$, and the study of angular correlations favors a $J^{P C}=0^{++}$assignment. At the beginning Belle named this state $Y(3940)$, then renamed to $Y(3915)$ after a more precise determination of the mass. The averaged mass and width are $M=(3918.4 \pm 1.9) \mathrm{MeV}$ and $\Gamma=(20 \pm 5) \mathrm{MeV}$. The PDG 2014 rebaptized to $\chi_{c 0}(2 P)$, choosing for an ordinary charmonium assignment for the state. However, the $\chi_{c 0}(2 P)$ is expected to have $\Gamma\left(\chi_{c 0}(2 P) \rightarrow D \bar{D}\right) \sim 30 \mathrm{MeV}$, i.e. wider than the total width measured of the $Y(3915)$. Even if no upper bound on $\mathcal{B}(Y(3915) \rightarrow D \bar{D})$ has been reported, no signs of a signal for such a decay appear in the measured $D \bar{D}$ invariant mass distributions for $B \rightarrow D \bar{D} K$ decays $[311,394]$. Moreover, the hyperfine splitting $\chi_{c 2}(2 P)-\chi_{c 0}(2 P)$ would be only $6 \%$ with respect to the $\chi_{c 2}(1 P)-\chi_{c 0}(1 P)$ splitting, much smaller than the same ratio in the bottomonium system $(r \sim 0.7)$, and than the potential model predictions [395] $(0.6<r<0.9)$. These facts challenge the ordinary charmonium interpretation [396, 397]. In the PDG 2015 online edition a compromise was reached, and it was called $\chi_{c 0}(3915)$ eventually. This state has been identified as the lightest $[c s][\bar{c} \bar{s}]$ tetraquark [139], although this interpretation looks at odds with the other states seen in $J / \psi \phi$ (see Section 8.6). Alternatively, Voloshin and Li have proposed a $D_{s}^{+} D_{s}^{-}$ molecular assignment [398].

$A \mathbf{X}(\mathbf{5 5 6 8}) ?$ - Recently $\mathrm{D} \varnothing$ claimed the discovery of a narrow state in the $B_{s}^{0} \pi^{ \pm}$invariant mass, with mass and width of $M=\left(5567.8 \pm 2.9_{-1.9}^{+0.9}\right) \mathrm{MeV}$ and $\Gamma=\left(21.9 \pm 6.4_{-2.5}^{+5.0}\right) \mathrm{MeV}$, and a significance of $3.9 \sigma$, increased to $5.1 \sigma$ if an additional cut $(\Delta R<0.3)$ is considered [133]. The ratio of the yield of resonant over background events in the detector acceptance region and $p_{\perp}>10 \mathrm{GeV}$ is $\rho=(8.6 \pm 1.9 \pm 1.4) \%$. The use of this additional cut has been criticized, even though the analysis was blind, and the cut was not optimized for this purpose. Right after, LHCb explored the very same channel, finding no hints of any resonant structure, neither at the mass observed by D $\varnothing$, nor all the way to the $B K$ threshold, and putting the $90 \%$ C.L. upper limit $\rho<1.6 \%$ in the detector acceptance region and $p_{\perp}>10 \mathrm{GeV}$ [282]. Even considering the differences between the two experiments in terms of acceptance, center-ofmass energy, and initial state, this gap makes the observation by $\mathrm{D} \varnothing$ less plausible. In Figure 47 we show the $B_{s}^{0} \pi^{ \pm}$ distributions measured by the two experiments. Neither the recent preliminary analysis by CMS is able to see the state [399]. It is worth noticing that such a state was at odds with many exotic models [11, 400-402].

$\boldsymbol{A} \boldsymbol{X ( 4 3 5 0 )}$ ? - Triggered by the observation of a $X(4140)$ resonance by CDF in $B \rightarrow K(J / \psi \phi))$, Belle explored the $\gamma \gamma \rightarrow J / \psi \phi$ channel. A peak with a $3.2 \sigma$ significance was seen at $M=\left(4350.6_{-5.1}^{+4.6} \pm 0.7\right) \mathrm{MeV}$ and $\Gamma=\left(13_{-9}^{+18} \pm 4\right) \mathrm{MeV}$, 

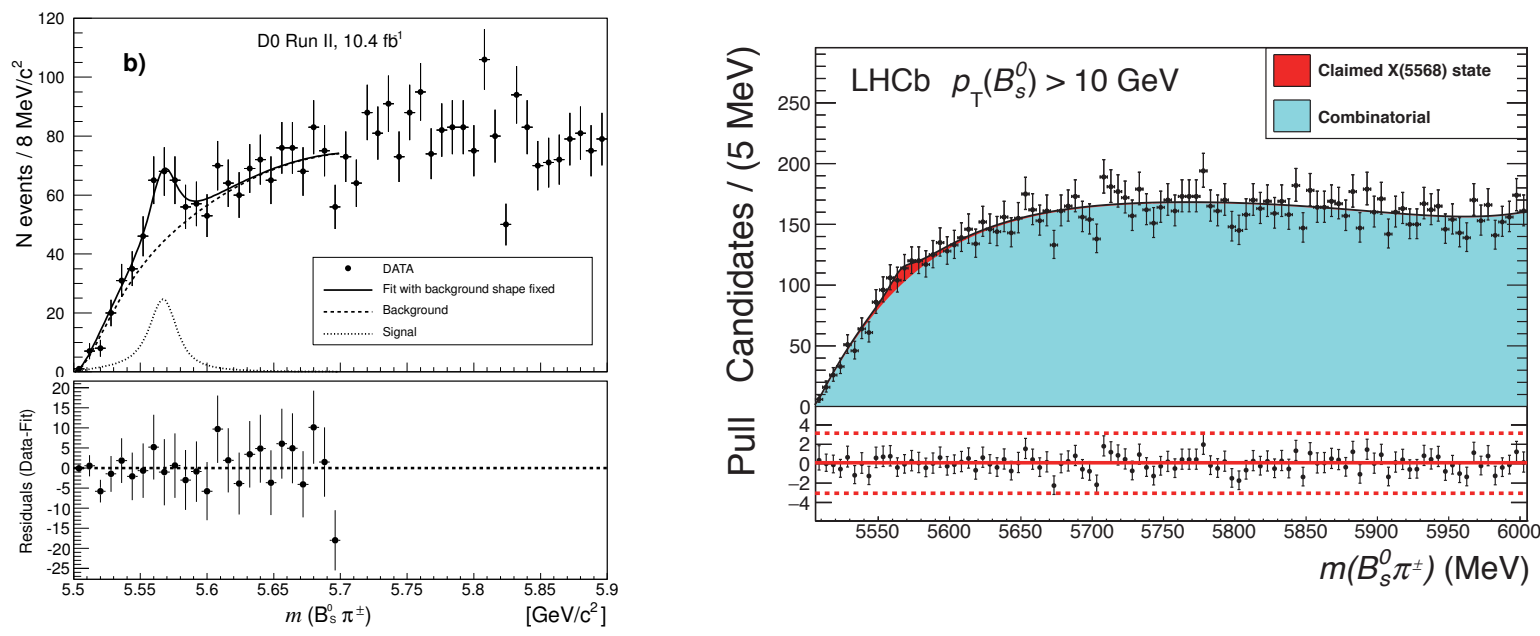

Figure 47: Left panel: The $m\left(B_{s}^{0} \pi^{ \pm}\right)$distribution together with the background distribution and the fit results, with $p_{\perp}>10 \mathrm{GeV}$ after applying the $\Delta R<0.3$ cone cut at $\mathrm{D} \varnothing$ [133]. Right panel: the same, with $p_{\perp}>10 \mathrm{GeV}$ with no cone cut, at LHCb [282]

and dubbed $X(4350)$. The narrow width and the signature $J \neq 1$ constrained by the production mode prevent the identification of this state with the other ones observed by LHCb in $B \rightarrow K(J / \psi \phi))$, see Section 8.6.

$\boldsymbol{X}(\mathbf{3 9 4 0})$ and $\boldsymbol{X ( 4 1 6 0 )}$ - The $X(3940)$ was observed by Belle in associated production with a $J / \psi$ with $M=$ $\left(3942_{-6}^{+7} \pm 6\right) \mathrm{MeV}$ and $\Gamma=\left(37_{-15}^{+26} \pm 8\right) \mathrm{MeV}[262,263]$. A partial reconstruction technique showed that $X(3940) \rightarrow D^{*} \bar{D}$ is the prominent decay mode, whereas $X(3940) \rightarrow D \bar{D}$ and $\rightarrow J / \psi \omega$ show no signal. The production mechanism $e^{+} e^{-} \rightarrow \gamma^{*} \rightarrow J / \psi X(3940)$ constrains the state to have $C=+$, and favors low values of the spin. The absence of the $D \bar{D}$ decay suggests unnatural parity, so the tentative $J^{P C}$ assignment for this state is $0^{-+}$.

The same analysis also observed a state called $X(4160)$ in the $D^{*} \bar{D}^{*}$ invariant mass. The fitted mass and width are $M=\left(4156_{-20}^{+25} \pm 15\right) \mathrm{MeV}$ and $\Gamma=\left(139_{-61}^{+111} \pm 21\right) \mathrm{MeV}$. Also in this case, the favored signature is $J^{P C}=0^{-+}$. These two states are good candidate for the $\eta_{c}(3 S)$ and $\eta_{c}(4 S)$ respectively, although their masses deviate from the ordinary predictions [403, 404].

$Z_{1}$ (4050) and $Z_{2}$ (4250) - In a Dalitz-plot analysis of $B \rightarrow \chi_{c 1} \pi^{+} K$ decays, Belle could get an acceptable fit only by adding two resonances in the $\chi_{c 1} \pi^{+}$channel, called $Z_{1}(4050)$ and $Z_{2}(4250)$ [265]. We report the Dalitz projection in Figure 48. The fitted masses and widths are $M_{1}=\left(4051 \pm 14_{-41}^{+20}\right) \mathrm{MeV}, \Gamma_{1}=\left(82_{-17-22}^{+21+47}\right) \mathrm{MeV}$, and $M_{2}=\left(4248_{-29-35}^{+44+180}\right) \mathrm{MeV}, \Gamma_{2}=\left(177_{-39-61}^{+54+316}\right) \mathrm{MeV}$, respectively. The same decay was investigated by BABAR, which carefully studied the effects of interference between resonances in the $K \pi$ system [266]. Considering interfering resonances in the $K \pi$ channel only, BABAR obtained good fits to data without adding any $\chi_{c} \pi$ resonance, but because of limited statistics, no strong conclusion could be made against the existence of these resonances. Their interpretation as $D$-wave tetraquarks has been suggested in a flux-tube model [405] Also, if one assumes a $0 / 2^{++}$assignment for the $Z_{1}$ (4050), the resonance can be identified as a member of the ground-state tetraquark multiplet — see Section 4.5 . Similarly, the $Z_{2}$ (4250) might be the radial excitation of the lightest $0^{++}$tetraquark.

$\boldsymbol{A} \mathbf{Z}(\mathbf{4 0 5 0}) ?$ - Belle report a $3.5 \sigma$ excess in the $\psi(2 S) \pi^{+}$invariant mass, in the $e^{+} e^{-} \rightarrow \psi(2 S) \pi^{+} \pi^{-}$[267], with best fit parameters $M=(4054 \pm 3 \pm 1) \mathrm{MeV}$ and $\Gamma=(45 \pm 11 \pm 6) \mathrm{MeV}$.

$Z$ (4200) - In the same analysis of the $B \rightarrow K \psi(2 S) \pi$ which confirmed the $Z(4430)$ (see Section 8.5), LHCb observed another significant broad structure, with mass and width $M=\left(4239 \pm 18_{-10}^{+45}\right) \mathrm{MeV}, \Gamma=\left(220 \pm 47_{-74}^{+108}\right) \mathrm{MeV}$, and likely $J^{P C}=0^{-+}$. Since the resonant nature in the Argand plot was unclear, LHCb prudently did not claim the 


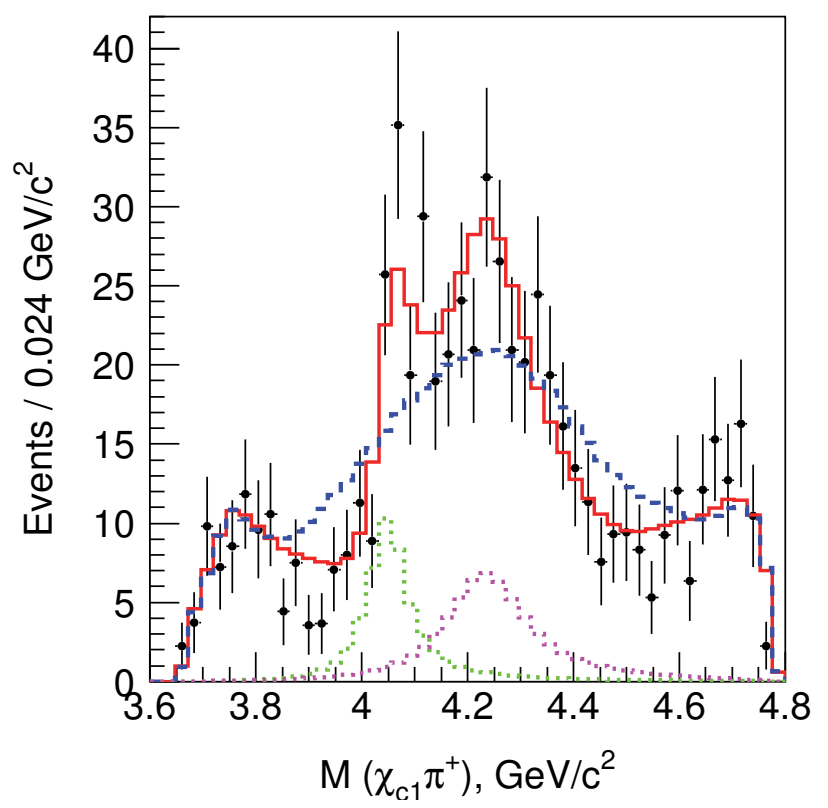

Figure 48: Invariant mass distributions of $\chi_{c 1} \pi^{ \pm}$, with fit results showing the charged resonances in the Belle [265] analysis. The region of the $K^{*}(890)$ and $K^{*}(1410)$ peaks are removed. In left panel, the solid red histogram shows the results of the fit that includes coherent $Z_{1}$ and $Z_{2}$ amplitudes; the dashed blue curve is the result of the fit using $K \pi$ amplitudes only.

discovery. Soon after, Belle claimed the discovery of a broad $Z(4200)$ state with likely $J^{P C}=1^{+-}$, mass and width $M=\left(4196_{-29-13}^{+31+17}\right) \mathrm{MeV}, \Gamma=\left(370_{-70-132}^{+70+70}\right) \mathrm{MeV}$, with a significance of $6.2 \sigma$. The two observations might be related to the same state, although such large widths cast some doubts on the actual resonant nature of these states. The interpretation as a tetraquark state has been suggested in the context of a flux-tube model [405], or with QCD sum rules [227].

\section{Conclusions and outlook}

A considerable part of the material presented in this review is selected from the vast literature on the $X Y Z P$ resonances, with the scope of preparing the discussion appearing in the core Sections in which we have much further elaborated on some new ideas published recently by our group. The main drive of this paper is to present a clear identification of the most striking conflicts of models with data and the indication of one (or more) possible ways to the solution of them. In some few cases we have reported, and set in the context, even some textbook results and derivations which we have used in diverse occasions. This was essentially done for the purpose of being self-contained.

The connection between the diquarkonium discrete spectrum and open meson thresholds might provide a set of 'selection rules' that fit the experimental data so far available. We presented some predictions and the rules for formulating them. Elements of a unitary picture are provided, although in the form of work in progress. The discussion about $X Y Z P$ states is certainly not concluded here, and more efforts, and hopefully new forthcoming data, are needed. Therefore, our hope is that the arguments formulated in this paper might attract and stimulate new research and fresh ideas also from outside the community which has traditionally worked in the field.

Even though we believe that no 'new physics' in the usual sense will be needed to definitely understand multiquark resonances, we cannot think that such a richness of experimental data and theoretical problems should pertain only to 
the practitioners of hadronic physics with a more or less high nuclear theory culture but could attract more attention in the high energy physics experimental/theoretical communities.

Coping and solving this problem could shed new light on non-perturbative QCD and in general on strong interactions, as documented in several valuable papers. We actually show here that, to some extent, this has already happened, and one of our aims was that of reporting on the diversity of ideas which have been advanced to solve the problems that data pose, and even to propose new directions of investigation which could arise exploiting methods proper of other branches of physics.

Model builders and lattice gauge theorists could find a very stimulating 'playground' in seeking solutions to the multiquark resonances problem, and this, in our opinion, is a concrete opportunity of progress in a field which is firmly connected to data and experimental physics practice.

As shown in the Sections more closely related to experiments, the complexity and variety of performed analyses is remarkable. Again this results from the work of a somewhat restricted community which we hope will benefit of more support and collaboration in the coming years.

In a few concluding words, we observe that diquark-antidiquark states, hybrids and molecules, in various forms, all catch important aspects of the problem, being understood that the observed multiquark resonances are not the mere manifestation of kinematical effects, like cusps, as some authors claim.

There might be several ways to learn from what was understood with all these methods, not loosing the contact to experimental evidence and preferring all those alternatives which appear to be formulated in a clearly falsifiable way. In a sense, this is a privilege of the field: theoretical models can be checked against data on reasonable time scales.

\section{Acknowledgements}

Most of the work presented here derives from invaluable collaboration with Luciano Maiani, Fulvio Piccinini and Veronica Riquer. They are all implicitly coauthoring this paper, exception made for what is imprecise or even wrong, which is our full responsibility. Along the years we benefited of the collaboration of Riccardo Faccini, who helped us to find the way in some experimental data analysis intricacies, and of a number of collaborators each of them contributing with their insight and work to solve the problems offered by the changing experimental picture - we wish to thank F. Brazzi, T. Burns, G. Cotugno, N. Drenska, G. Filaci, A. L. Guerrieri, M. Papinutto, V. Prosperi, C. Sabelli, and N. Tantalo. ADP benefited from sporadic collaborations and discussions with A. Ali, I. Bigi, B. Grinstein, and R. Lebed and recent exchanges with G. C. Rossi, G. Veneziano, and S. Peris. AP wishes to thank M. Bochicchio and A. Szczepaniak for many fruitful discussions. The support and dialogue with experimentalists has been constant. We wish to thank S. Stone for several decisive exchanges, together with A. A. Alves, M. Battaglieri, G. Cavoto, M. Destefanis, R. Mussa, A. Palano, M. Pappagallo, A. Pompili, F. Renga, and U. Tamponi. The confrontation with the ideas of E. Braaten and M. Voloshin has been very instructive to us. This material is based upon work supported in part by the U.S. Department of Energy, Office of Science, Office of Nuclear Physics under contract DE-AC0506OR23177, and DE-FG02-11ER41743. 


\section{Appendix A. $X(3872)$ production at hadron colliders}

It is possible to estimate an upper bound for the production cross section of the $X(3872)$ as follows [31]

$$
\sigma(p \bar{p} \rightarrow X(3872)) \lesssim \sigma^{\max }\left(p \bar{p} \rightarrow D^{0} \bar{D}^{* 0}\right) \sim \int_{|\boldsymbol{k}| \in \mathcal{R}}\left|\left\langle D^{0} \bar{D}^{* 0}(\boldsymbol{k}) \mid p \bar{p}\right\rangle\right|^{2}
$$

where $\boldsymbol{k}$ is the relative momentum between the two $D$ mesons in their center of mass and $\mathcal{R}$ is the domain where the two-body wave function for the molecular $X(3872)$ is significantly different from zero.

Such an upper bound can be estimated by counting the number of $D^{0} \bar{D}^{* 0}$ produced with a relative momentum lower than a certain $k_{0}$ value. This has been done [31] using HERWIG [406] and PYTHIA [407], taking $\mathcal{R}$ to be a ball or radius $[0,35] \mathrm{MeV}$, on the basis of a naïve gaussian shape for the two-body wave function of the $X$.

Next we assume that all these pairs will convert into $X(3872)$.

The result of the MC simulation (with a MC luminosity of $\sim 100 \mathrm{nb}^{-1}$ ) was a maximum production cross section of $0.071 \mathrm{nb}$ for HERWIG and $0.11 \mathrm{nb}$ for PYTHIA, which are both smaller than the experimental value $(\sim 30 \mathrm{nb})$ by more than two orders of magnitude. This seemed to be the definitive proof of the inconsistency of the molecular interpretation with the experimental data.

However, the previous approach was later criticized in [36] and it was shown that the theoretical and experimental cross sections might be matched resorting to Final State Interactions (FSI) [408]. The possible presence of FSI, in fact, casts doubts on the applicability of the simple coalescence picture to the case of the $X(3872)$, since the two components of the molecule could be bound by final state rescattering even if their relative center of mass momentum is large. In particular, the Migdal-Watson theory would change the previous results in two different ways

1. The cross section for the production of the $X$ should be modified to

$$
\sigma(p \bar{p} \rightarrow X(3872)) \simeq\left[\sigma\left(p \bar{p} \rightarrow D^{0} \bar{D}^{* 0}\right)\right]_{k_{0}<k_{0}^{\max }} \times \frac{6 \pi \sqrt{-2 \mu E_{X}}}{\Lambda}
$$

where $\left[\sigma\left(p \bar{p} \rightarrow D^{0} \bar{D}^{* 0}\right)\right]_{k_{0}<k_{0}^{\max }}$ is the upper bound evaluated in (A.1) and $\Lambda \sim m_{\pi}$ is the typical range of the interaction between the components;

2. Instead of being taken as the inverse of the spread of the spatial wave function, the maximum value for the relative momentum should be given by the inverse of the range of the interaction, $k_{0}^{\max } \simeq c \Lambda$, with $c=O(1)$.

By setting $k_{0}=2.7 \Lambda \simeq 360 \mathrm{MeV}$ one can increase the theoretical cross section up to $32 \mathrm{nb}$, which is in agreement with the experimental value.

This approach, left alone, has some flaws [32]: it can be shown that the use of Eq. (A.2) should enhance the occurrence of a new hypothetical molecule, the $D_{s}^{+} D_{s}^{*-}$, which otherwise would be suppressed, as one could infer by looking at data on $D_{s}$ production at Tevatron [409]. In fact, the theoretical production cross section for this $X_{s}$ would be $\sigma \simeq 1 \div 3 \mathrm{nb}$ and should be detected by the CDF experiment. No hint for such a particle has been found. Furthermore, the applicability of the Migdal-Watson theorem requires that, $i$ ) the two final particles should be in an $S$-wave state and $i$ ) they should be free to interact with each other up to relative distances comparable to the interaction range.

The inclusion of relative momenta up to $k_{0}^{\max } \simeq 360 \mathrm{MeV}$ means to include relative orbital angular momenta up to $\ell \sim k_{0}^{\max } / m_{\pi} \simeq 2 \div 3$, thus violating the hypothesis $i$ ). Moreover, using again the MC softwares HERWIG and PYTHIA, one can show [32] that in high energy collisions, such as those occurring at Tevatron and LHC, there are 


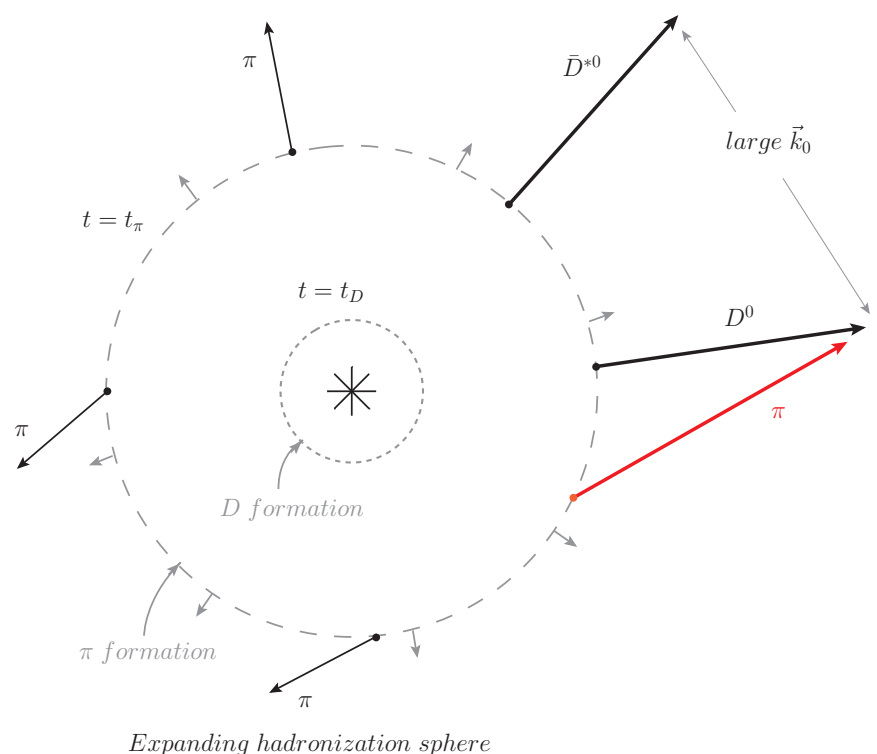

$(a)$

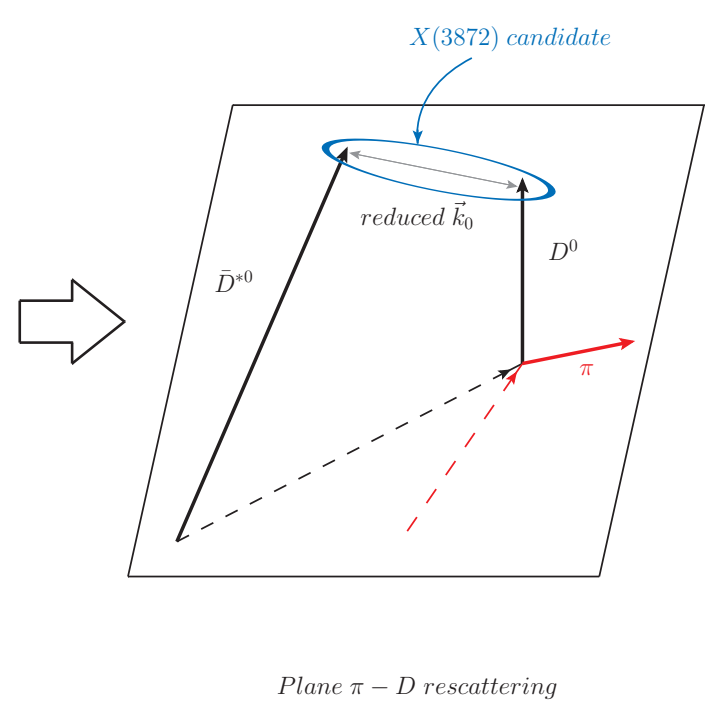

$(b)$

Figure A.49: Pictorial representation of the rescattering mechanism. After the main high-energy interaction has taken place, the final state particles can be thought of as belonging to an expanding sphere. The hadronization time of a certain particle goes as $t_{\text {hadr }} \propto 1 / m$. Therefore the $D$ mesons hadronize at an earlier time $t_{D}$ whereas pions hadronize at a later time $t_{\pi}$ (dotted and dashed spheres respectively). In figure (a) the $D^{0} \bar{D}^{* 0}$ pair starts with a large relative momentum $\boldsymbol{k}_{0}$. However, the $D^{0}$ might interact with one of the comoving pions (red arrow). The $\pi-D$ rescattering (figure (b)) can deviate the $D^{0}$ and reduce the relative momentum $\boldsymbol{k}_{0}$ thus producing a possible $X(3872)$ candidate.

on average $2 \div 3$ more hadrons having a relative momentum with respect to one of the two components smaller that $100 \mathrm{MeV}$; this extra hadron 'pollution' challenges the hypothesis ii).

Even though the presence of other hadrons (mainly pions) surrounding the system might not allow the use of FSI, it might have played an important role at explaining the unnaturally high prompt production of the $X(3872)$.

It has been proposed [20] that the possible elastic scattering of "comoving" pions with one of the components of the molecule might decrease their relative momentum, hence increasing the number of would-be molecules. The idea is that the interaction might push the pair both to higher and to lower values of $k_{0}$. However, since the majority of would-be molecules are produced with high relative momenta, even if a small fraction of them would be pushed to smaller momenta, that could cause a feed-down of pairs towards the lower bins of the distribution, where the $X(3872)$ candidates should be found. For a pictorial representation of the considered rescattering mechanism see Figure A.49.

It is worth noting that, if we assume the initial total energy $E$ of the pair to be positive, the decrease in $k_{0}$ due to elastic scattering may even bring $E$ to negative values, hence assuring the binding of the molecule in a deuteronlike state (see Section 3.3). Therefore, in this model the $X(3872)$ would be a genuine, negative energy $D^{0} \bar{D}^{* 0}$ bound state, whose lifetime would be entirely regulated by the lifetime of its shorter lived component, the $\bar{D}^{* 0}$. Hence, this mechanism also predicts a narrow width, $\Gamma_{X} \sim \Gamma_{D^{*}} \simeq 65 \mathrm{keV}$. Thus, when considering the interactions with comoving pions there are two possibilities

1. The energy $E$ in the center of mass is decreased so that to meet the condition $E \sim B$, where $B$ is the close-tothreshold discrete level discussed in Section 2;

2. Because of the interaction with a third body (the pion) of one of the constituents of the would-be-molecule, $E$ becomes small and negative: a deuteron-like state is formed.

The interaction in the final state of the molecular constituents with pions has been studied in [20, 30] with MC 


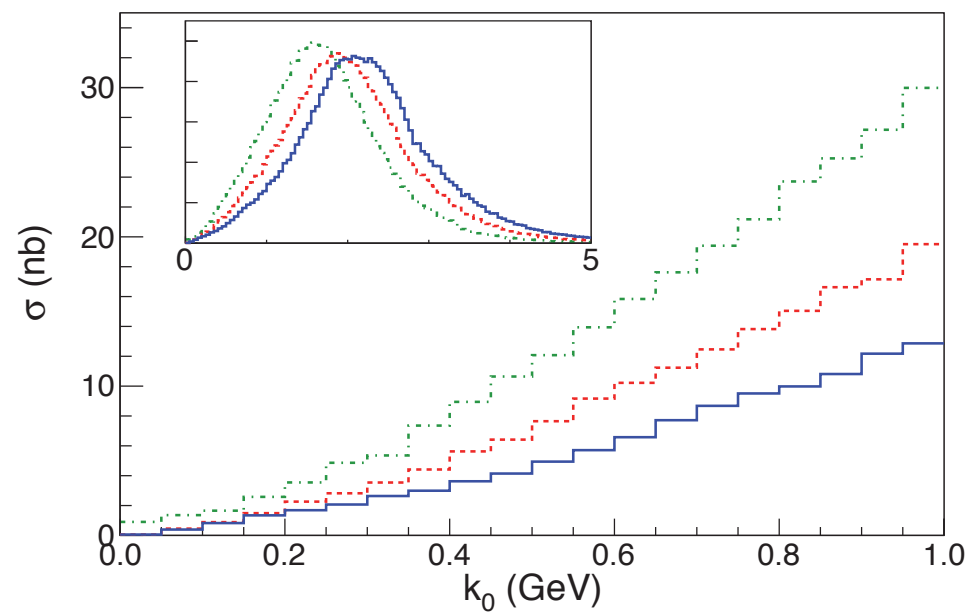

Figure A.50: Integrated cross section of $D^{0} \bar{D}^{* 0}+$ h.c. pairs at CDF obtained with HERWIG, without (blue, solid), with one (red, dashed) and with three (green, dot-dashed) interactions with pions, from [30]. In the inset the same plot on a wider range of $k_{0}$ values.

methods. The recipe used is as follows: the 10 most coplanar pions to the $D^{0} \bar{D}^{* 0}$ plane are selected, then the pion which will interact with (say the $D^{0}$ ) is randomly chosen and lastly the most parallel pion to the non-interacting meson (say the $\bar{D}^{* 0}$ ) is selected. One expects this configuration to be the most effective in physical events. Moreover, in order to prevent that $D$ mesons belonging to different jets (separated in coordinate space) would get closer by the scattering with a hard pion, one also requires $\Delta R_{D D^{*}} \equiv \sqrt{\left(\Delta y_{D D^{*}}\right)^{2}+\left(\Delta \phi_{D D^{*}}\right)^{2}}<0.7$.

It has been checked [30] that this mechanism does not spoil the high energy behavior of the relevant $D$ meson distribution. It was actually showed that the inclusion of one elastic scattering improves the agreement of the simulation with experimental data. This is a strong hint of the fact that this mechanism actually takes place in real physical events and should hence be considered when studying final hadronic distributions.

As one can see from Figure A.50, the proposed mechanism is actually effective in feeding down the lower $k_{0}<$ $50 \mathrm{MeV}$ bin. It is also possible to estimate how many of these interactions may take place. In particular, considering a model where all the produced hadrons are flying away from each other on the surface of a sphere and taking into account the range of the interaction, one finds [20] that the simulations suggest an average of 3 scatterings per event. These consecutive interactions can be reproduced by implementing a Markov chain [20].

If one trusts the coalescence model for the $X(3872)$ and hence consider $k_{0}^{\max } \simeq 50 \mathrm{MeV}$, not even the elastic scattering with three consecutive pions is able to enhance the production cross section up to the experimental one $(\sigma \simeq$ $30 \mathrm{nb}$ ). Moreover, if one considers the use of FSI [36, 37] as explained previously, then it should be $k_{0}^{\max } \simeq 360 \mathrm{MeV}$. With this integration region, the simulations produce a cross section after the interaction with one pion - and after a rescaling needed to take into account the different normalization factors between the two works [30, 37]- that is equal to $\sigma(1 \pi) \simeq 52 \mathrm{nb}$, even larger than the experimental one!

In Table A.17 we report the values of the integrated cross section for the production of the $X(3872)$ varying both the number of interacting pions and the maximum $k_{0}$ allowed for the pair.

To summarize, the experimental value of the prompt production cross section of the $X(3872)$ casted serious doubts on a simplified interpretation in terms of a $D^{0} \bar{D}^{* 0}$ molecule. According to the expectations following from the phenomenological coalescence model, the production of such a weakly bound state should be strongly suppressed in 


\begin{tabular}{c|ccc}
\hline \hline$k_{0}^{\max }$ & $50 \mathrm{MeV}$ & $300 \mathrm{MeV}$ & $450 \mathrm{MeV}$ \\
\hline$\sigma(0 \pi)$ & $0.06 \mathrm{nb}$ & $6 \mathrm{nb}$ & $16 \mathrm{nb}$ \\
$\sigma(1 \pi)$ & $0.06 \mathrm{nb}$ & $8 \mathrm{nb}$ & $22 \mathrm{nb}$ \\
$\sigma(3 \pi)$ & $0.9 \mathrm{nb}$ & $15 \mathrm{nb}$ & $37 \mathrm{nb}$ \\
\hline \hline
\end{tabular}

Table A.17: Effect of multiple scatterings in $X(3872)$ production cross section. $k_{0}^{\max }$ indicates the integration region $0<k_{0}<k_{0}^{\max }$ ].

high energy collisions. Even though many ideas and models have been proposed during the years none of them has successfully reconciled the theoretical expectations with the experimental results.

It should also be emphasized that the inclusion of possible interactions between comoving pions and final state mesons $[20,30]$ turned out to improve the accordance between the simulated MC distributions and the experimental ones.

\section{Appendix B. Diquarks in SU(N)}

Consider two quarks interacting through the exchange of one virtual gluon in $N=3$ QCD as in Figure B.51

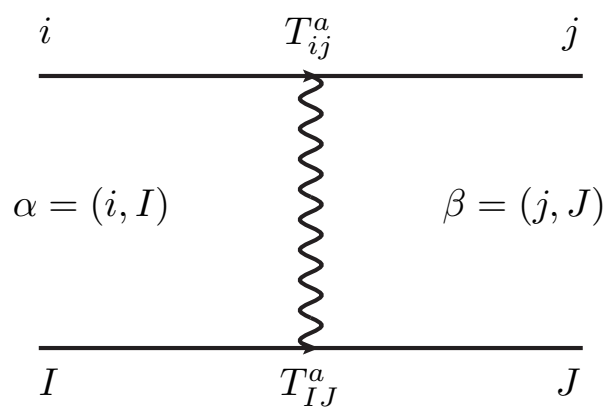

Figure B.51: One-gluon exchange interaction.

The $T_{i j}^{a} T_{I J}^{a}$ tensor product can be mapped into a $9 \times 9$ matrix whose entries $A_{\alpha \beta}$ correspond to the 81 possible combinations of initial and final colors as in Figure B.51. The $v$ eigenvectors of $A$ identify 3 antisymmetric color configurations and 6 symmetric ones. For each $v$ the $v^{T} A v$ product is a superposition of the color diagrams in Figure B.51 defining amplitudes which are (anti-)symmetric under the simultaneous exchange of the colors $i \rightarrow I, j \rightarrow J$.

Each of these 9 color configurations is weighted by a coefficient $h$, the eigenvalue related to $v$. The $h$ 's are found to be negative in the antisymmetric cases and positive in the symmetric ones: $h=-2 / 3$ and $h=1 / 3$ respectively for SU(3). The value of $h$ corresponds to the product of charges in a abelian theory - thus we get repulsion in the symmetric eigenchannels ${ }^{57}$ and attraction in the antisymmetric ones.

The eigenvalues $h$ are more conveniently computed through the quadratic Casimirs of the irreducible representations $\boldsymbol{S}_{i}$ obtained from the Kronecker decomposition of the product $\boldsymbol{R}_{1} \otimes \boldsymbol{R}_{2}=\boldsymbol{S}_{1} \oplus \boldsymbol{S}_{2} \oplus \ldots$. In the case of quark-quark

\footnotetext{
${ }^{57}$ To mean $i=I, j=J$.
} 
interaction in SU(3), $\boldsymbol{R}_{1}=\boldsymbol{R}_{2}=\mathbf{3}$ and $\boldsymbol{S}_{1}=\overline{\mathbf{3}}, \boldsymbol{S}_{2}=\mathbf{6}$. The formula for the eigenvalues $h_{i}$ in the various eigenchannels is in general

$$
h_{i}=\frac{1}{2}\left(C_{S_{i}}-C_{\boldsymbol{R}_{1}}-C_{\boldsymbol{R}_{2}}\right)
$$

where $C_{\boldsymbol{S}_{i}}, C_{\boldsymbol{R}_{1}}, C_{\boldsymbol{R}_{2}}$ are the quadratic Casimirs in the $\boldsymbol{S}_{i}, \boldsymbol{R}_{1}, \boldsymbol{R}_{2}$ representations respectively.

In the generic case of $\mathrm{SU}(\mathrm{N})$ we have that

$$
N \otimes N=\frac{N(N-1)}{2} \oplus \frac{N(N+1)}{2}
$$

where $N(N-1) / 2$ is antisymmetric and $N(N+1) / 2$ is symmetric.

The Casimirs associated to these representations are given in the following table

\begin{tabular}{c|cc}
\hline \hline Representation $\boldsymbol{R}$ & $C_{\boldsymbol{R}}$ & $h$ \\
\hline $\boldsymbol{N}$ & $\left(N^{2}-1\right) /(2 N)$ & - \\
$\boldsymbol{N}(\boldsymbol{N}+\mathbf{1}) / \mathbf{2}$ & $(N-1)(N+2) / N$ & $(N-1) / 2 N>0$ \\
$\boldsymbol{N}(\boldsymbol{N}-\mathbf{1}) / \mathbf{2}$ & $(N+1)(N-2) / N$ & $-(N+1) / 2 N<0$ \\
\hline \hline
\end{tabular}

Table B.18: Quadratic Casimir operators for the fundamental, the two index symmetric and antisymmetric representations, in color SU(N), $N \geq 2$. In the third column, the coefficient of the potential energy for color symmetric and antisymmetric diquarks in the one-gluon exchange approximation. Attraction in the antisymmetric channel persists at large $N$.

In the singlet channel of $N \otimes \bar{N}$, the attraction is weighted by $h=-\left(N^{2}-1\right) / 2 N$. Therefore the singlet channel is $(N-1)$ more attractive than the antisymmetric $N(N-\mathbf{1}) / 2$ channel reported in Table B.18, in SU(3). In the one-gluon exchange approximation, the singlet channel in $q \bar{q}$ is (just) twice more attractive than the color antitriplet channel in $q q$.

Values in Tables B.18 can be obtained starting from formula for the diagonalization of the tensor product in SU(N)

$$
T_{R_{1}}^{a} \otimes T_{R_{2}}^{a}=\bigoplus_{i} \frac{1}{2}\left(C_{S_{i}}-C_{R_{1}}-C_{R_{2}}\right) \mathbb{1}_{S_{i}}
$$

Take $S_{1}=N(N-\mathbf{1}) / 2, S_{2}=N(N+\mathbf{1}) / 2$ and $C_{1}=C_{S_{1}}, C_{2}=C_{S_{2}}$. The trace of (B.3) gives

$$
\frac{1}{2} x \frac{N(N-1)}{2}+\frac{1}{2} y \frac{N(N+1)}{2}=0
$$

where

$$
\begin{aligned}
& x=\left(C_{1}-2 C_{N}\right) \\
& y=\left(C_{2}-2 C_{N}\right)
\end{aligned}
$$

and

$$
C_{N}=\frac{N^{2}-1}{2 N}
$$

For the $N$ of SU(N) we have the trace result

$$
T_{i j}^{a} T_{j k}^{b} T_{k r}^{a} T_{r i}^{b}=\left(C_{N}-\frac{1}{2} N\right) \frac{N^{2}-1}{2}=-\frac{1}{4}\left(\frac{N^{2}-1}{N}\right)
$$


which can be written as a product of tensor products

$$
\left(T_{i j}^{a} T_{k r}^{a}\right)\left(T_{j k}^{b} T_{r i}^{b}\right)
$$

This can be mapped in the form

$$
A_{i k, j r} A_{j r, k i}
$$

which in turn becomes a trace by inverting $k i$ with $i k$.

Recalling the Fierz identity

$$
T_{i r}^{a} T_{k j}^{a}-\frac{N-1}{2 N} \delta_{i r} \delta_{k j}=\frac{N-1}{2 N} \delta_{i j} \delta_{k r}-T_{i j}^{a} T_{k r}^{a}
$$

The term in (B.8) can be rewritten as

$$
\left(T_{i j}^{a} T_{k r}^{a}\right)\left(-T_{j i}^{b} T_{r k}^{b}+\frac{N-1}{2 N} \delta_{j k} \delta_{r i}\right)
$$

where the term neglected in parentheses gives zero. The latter, containing a term in the form $A_{i k, j r} A_{j r, i k}$ is a trace and can be re-written as

$$
-\frac{1}{4} x^{2} \frac{N(N-1)}{2}-\frac{1}{4} y^{2} \frac{N(N+1)}{2}+\frac{N-1}{2 N} \frac{N^{2}-1}{2}
$$

Plugging this in the lhs of (B.7) we get

$$
-\frac{1}{4} x^{2} \frac{N(N-1)}{2}-\frac{1}{4} y^{2} \frac{N(N+1)}{2}=-\frac{1}{4}\left(N^{2}-1\right)
$$

From

$$
y=-x \frac{N-1}{N+1}
$$

as obtained by (B.4), we finally get

$$
x^{2}\left(\frac{N(N-1)}{2}+\frac{N(N-1)^{2}}{2(N+1)}\right)=\left(N^{2}-1\right)
$$

with solutions

$$
x= \pm \frac{N+1}{N}
$$

The negative sign solution gives

$$
C_{1}=\frac{(N+1)(N-2)}{N}
$$

In the case of $\mathrm{SU}(3), R_{1}=\overline{\mathbf{3}}$ and

$$
C_{1}=C_{\overline{\mathbf{3}}}=\frac{4}{3}
$$

Computing $y$ from (B.14) we get

$$
C_{2}=\frac{(N-1)(N+2)}{N}
$$

In the case of $\mathrm{SU}(3) R_{2}=\mathbf{6}$ and

$$
C_{2}=C_{6}=\frac{10}{3}
$$




\section{Appendix C. Interaction Hamiltonian between open and closed channels in strong interactions}

We will now describe the formalism used to derive the interaction Hamiltonian between the open and closed channels. It first appeared in cold atom physics in [410, 411] (see [22, 23] for a textbook treatment). Suppose that $Q$ is is the projection operator on the space of diquarkonia $\Psi_{d}$ and $\mathcal{P}$ is the one on the space of open charm/beauty meson-meson free states $\Psi_{m}$. Let $\Psi_{m}$ be the molecular threshold closer, from below, to the mass of the $\Psi_{d}$ state.

Assume the orthogonality of $P$ and $Q$ spaces

$$
Q \mathcal{P}=\mathcal{P} Q=0
$$

Let $H$ be the strong interaction Hamiltonian which determines the dynamics of the hadronization state $\Psi$ in (6.1), which we can more shortly write as $\Psi=\Psi_{Q}+\Psi_{P}=(\mathcal{P}+Q) \Psi$, since $(\mathcal{P}+Q)=\mathbb{1}$. Then

$$
H \Psi=E \Psi
$$

can be projected into $[23,411]$

$$
\begin{aligned}
\left(E-H_{P P}\right) \Psi_{P} & =H_{P Q} \Psi_{Q} \\
\left(E-H_{Q Q}\right) \Psi_{Q} & =H_{Q P} \Psi_{P}
\end{aligned}
$$

with the notation of compact hadron $\Psi_{Q}$ states (diquarkonia) and free meson-meson states $\Psi_{P}$. The $H_{P Q}$ and $H_{Q P}$ Hamiltonians represent the couplings between states in the $P$ and $Q$ sub-spaces

$$
H_{P P}=\mathcal{P} H \mathcal{P} \quad H_{Q Q}=Q H Q \quad H_{P Q}=\mathcal{P} H Q \quad H_{Q P}=H_{P Q}^{\dagger}=Q H \mathcal{P}
$$

Eq. (C.3b) has the formal solution (compare to (2.7))

$$
\Psi_{Q}=\frac{1}{E-H_{Q Q}+i \epsilon} H_{Q P} \Psi_{P}
$$

Despite the $+i \epsilon$ prescription, we do not actually distinguish between in- and out- $\left(\Psi^{ \pm}\right)$scattering states as we assume to be at very low wave numbers $k$ where there are no $e^{ \pm i k r}$ factors in the wave-mechanics description. The solution (C.5) can be plugged back into (C.3a) giving

$$
\left(E-H_{P P}-H_{P P}^{\prime}\right) \Psi_{P}=0
$$

where

$$
H_{P P}=H_{0}+V_{1}
$$

$H_{0}$ is the kinetic energy of the relative motion and $V_{1}$ are interactions in the $P$ space which are present also in absence of $Q$ levels. $H_{P P}^{\prime}$ interactions are due instead to a spectrum of compact hadron states (diquarkonia) in the $Q$ space. The formal expression of $V_{I}$ is

$$
V_{I} \equiv H_{P P}^{\prime}=H_{P Q} \frac{1}{E-H_{Q Q}+i \epsilon} H_{Q P}
$$

and represents an effective interaction in the $P$ space which exist only because of discrete levels in the $Q$ space (one is enough!) being immersed in the continuum spectrum of $P$. It is the existence of such a discrete level which allows $P \rightarrow Q \rightarrow P$ transitions regulated by a non-local potential in the space of meson-meson states ${ }^{58}$. $H_{P Q}$ and $H_{Q P}$ might

\footnotetext{
${ }^{58}$ At a higher order of perturbation theory it is found

$$
H_{Q Q}^{\prime}=H_{Q P} \frac{1}{E-H_{P P}+i \epsilon} H_{P Q}
$$
}

which means that the interaction between the two channels shifts the levels in the closed channel. 
be very small, as can be inferred from the discussion of the large- $N$ QCD expansion of Section 5.2.2. It is only the tuning of $E$ with the diquarkonium level which might abruptly enhance $V_{I}$ and produce the observed resonance effect.

Because of this, scattering in $P$ space has a term in the $T$-matrix

$$
T_{\alpha \alpha} \sim \frac{\left|\left\langle\Psi_{n} \mid H_{Q P} \Psi_{\alpha}\right\rangle\right|^{2}}{E_{\alpha}-E_{n}}
$$

where

$$
E_{n} \Psi_{n}=H_{Q Q} \Psi_{n}
$$

are the levels describing diquarkonia in $Q$ space. Eq. (C.10) should be confronted to Eq. (2.18) noting that in the case of Eq. (2.18) the discrete level is found in the negative energy spectrum of some potential $V$ whereas in the case of (C.10) the discrete level is on the same side of the continuum spectrum, but pertains to a different potential with respect to that giving the onset of continuum levels. This strongly differentiates our metastable state from a true resonance. The latter is above threshold but belongs to the same potential as for the two-meson state.

$H_{Q Q}$ can be described in the constituent quark model picture by

$$
H_{Q Q}=H_{0}+V_{2}
$$

where $H_{0}$ is the kinetic term of diquarks — compare with (C.7).

\section{Appendix D. The effective hadroquarkonium Hamiltonian}

In this section we will give a more rigorous derivation of the effective Hamiltonian (7.1) for the interaction between a compact quarkonium state and the gluonic field generated by the light matter around it. We will mostly follow the procedure in $[150,151]$. The full Hamiltonian of a system with an heavy quark pair $Q \bar{Q}$ and a gluonic field can be written as

$$
H=H_{Q}+H_{g}+H_{\text {int }} \equiv H_{0}+H_{\text {int }}
$$

where $H_{Q}$ and $H_{g}$ describe the $Q \bar{Q}$ and the gluonic system in absence of their mutual interaction. The QCD multipole expansion for $H_{\text {int }}$ produces the leading terms [150-153]

$$
H_{1}=T^{a} V^{a} \quad \text { and } \quad H_{2}=-\frac{1}{2} \Delta T^{a} \boldsymbol{r} \cdot \boldsymbol{E}^{a}
$$

with $T^{a}=T_{Q}^{a}+T_{\bar{Q}}^{a}, \Delta T^{a}=T_{Q}^{a}-T_{\bar{Q}}^{a}$ and $\boldsymbol{E}^{a}$ is the chromoelectric field generated by the light matter. The exact form of $V^{a}$ does not concern us as it will not give any contribution. $H_{1}$ corresponds to the "charge" operator while $H_{2}$ to the dipole. It should also be noted that both $V^{a}$ and $\boldsymbol{E}^{a}$ only act on the light degrees of freedom.

Consider now two states, $\left|n_{1}\right\rangle$ and $\left|n_{2}\right\rangle$ composed by a quarkonium, $\left|\psi_{i}\right\rangle$, and a light state, $\left|\phi_{i}\right\rangle$. In absence of interaction the complete state is simply the tensor product $\left|n_{i}\right\rangle=\left|\psi_{i}\right\rangle\left|\phi_{i}\right\rangle$. The amplitude $n_{1} \rightarrow n_{2}$ is then given by

$$
\mathcal{A}=\sum_{k=0}^{\infty}\left\langle n_{1}\left|H_{\text {int }} \frac{1}{E_{n_{1}}-H_{0}}\left(H_{\text {int }} \frac{1}{E_{n_{1}}-H_{0}}\right)^{k} H_{\text {int }}\right| n_{2}\right\rangle \approx\left\langle n_{1}\left|H_{\text {int }} \frac{1}{E_{n_{1}}-H_{0}} H_{\text {int }}\right| n_{2}\right\rangle=\left\langle n_{1}\left|H_{2} \frac{1}{E_{n_{1}}-H_{0}} H_{2}\right| n_{2}\right\rangle
$$

where the first order transition is forbidden by color and in the last step we used the fact that $H_{1}$ always vanishes on color singlet states. We can now introduce a complete sum over the eigenstates of $H_{0},|m\rangle=\left|m_{Q}\right\rangle\left|m_{g}\right\rangle$, where $\left|m_{Q}\right\rangle$ is 
a quarkonium eigenstate of $H_{Q}$ and $\left|m_{g}\right\rangle$ is a gluonic excitation, eigenstate of $H_{g}$. We obtain

$$
\begin{aligned}
\mathcal{A} & =\sum_{m}\left\langle n_{1}\left|\frac{1}{2} \Delta T^{a} r_{i} E_{i}^{a} \frac{|m\rangle\langle m|}{E_{n_{1}}-H_{0}} \frac{1}{2} \Delta T^{b} r_{j} E_{j}^{b}\right| n_{2}\right\rangle \\
& =\sum_{m_{Q}, m_{g}}\left\langle\phi_{1}\left|E_{i}^{a}\right| m_{g}\right\rangle\left\langle m_{g}\left|E_{j}^{b}\right| \phi_{2}\right\rangle\left\langle\psi_{1}\left|\frac{1}{2} \Delta T^{a} r_{i} \frac{\left|m_{Q}\right\rangle\left\langle m_{Q}\right|}{E_{n_{1}}-E_{m_{Q}}-E_{m_{g}}} \frac{1}{2} \Delta T^{b} r_{j}\right| \psi_{2}\right\rangle
\end{aligned}
$$

Note that since $\Delta T^{a}$ transforms a color singlet into an octet, the only states $\left|m_{Q}\right\rangle$ surviving in the sum are color octet. We will now make the approximation $E_{m_{g}} \ll E_{n_{1}}-E_{m_{Q}}$, i.e. very soft gluonic mode. This is not necessarily a good approximation as shown in [151] but it considerably simplifies the computation. The most general result can be found in $[152,153]$. We then have

$$
\begin{aligned}
\mathcal{A} & \approx \sum_{m_{Q}, m_{g}}\left\langle\phi_{1}\left|E_{i}^{a}\right| m_{g}\right\rangle\left\langle m_{g}\left|E_{j}^{b}\right| \phi_{2}\right\rangle\left\langle\psi_{1}\left|\frac{1}{2} \Delta T^{a} r_{i} \frac{\left|m_{Q}\right\rangle\left\langle m_{Q}\right|}{E_{n_{1}}-E_{m_{Q}}} \frac{1}{2} \Delta T^{b} r_{j}\right| \psi_{2}\right\rangle \\
& =\left\langle\phi_{1}\left|E_{i}^{a} E_{j}^{b}\right| \phi_{2}\right\rangle \sum_{m_{Q}}\left\langle\psi_{1}\left|\frac{1}{2} \Delta T^{a} r_{i} \frac{\left|m_{Q}\right\rangle\left\langle m_{Q}\right|}{E_{n_{1}}-E_{m_{Q}}} \frac{1}{2} \Delta T^{b} r_{j}\right| \psi_{2}\right\rangle \\
& =-\left\langle\phi_{1}\left|E_{i}^{a} E_{j}^{b}\right| \phi_{2}\right\rangle\left\langle\psi_{1}\left|\frac{1}{2} \Delta T^{a} r_{i} G_{(8)} \frac{1}{2} \Delta T^{b} r_{j}\right| \psi_{2}\right\rangle
\end{aligned}
$$

In the last step we used the fact that $G_{(8)}=\sum_{m_{Q}} \frac{\left|m_{Q}\right\rangle\left\langle m_{Q}\right|}{E_{m_{Q}}-E_{n_{1}}}$ is the Green's function of a heavy quark pair in a color octet configuration. Since the states $\left|\phi_{i}\right\rangle$ are both color singlets, color and Lorentz invariance imply

$$
\left\langle\phi_{1}\left|E_{i}^{a} E_{j}^{b}\right| \phi_{2}\right\rangle=\frac{1}{8} \delta_{a b} \frac{1}{3} \delta_{i j}\left\langle\phi_{1}\left|\boldsymbol{E}^{c} \cdot \boldsymbol{E}^{c}\right| \phi_{2}\right\rangle
$$

and therefore

$$
\mathcal{A} \approx-\frac{1}{96}\left\langle\phi_{1}\left|E_{j}^{b} E_{j}^{b}\right| \phi_{2}\right\rangle\left\langle\psi_{1}\left|\Delta T^{a} r_{i} G_{(8)} \Delta T^{a} r_{i}\right| \psi_{2}\right\rangle
$$

Therefore, if we integrate out the quarkonium states and only leave the effective Hamiltonian for the light degrees of freedom, we arrive again to Eq. (7.1)

$$
H_{\mathrm{eff}}=-\frac{1}{2} \alpha^{\left(\psi_{1} \psi_{2}\right)} E_{i}^{a} E_{i}^{a}
$$

where we defined the chromo-polarizability as

$$
\alpha^{\left(\psi_{1} \psi_{2}\right)}=\frac{1}{48}\left\langle\psi_{1}\left|\Delta T^{a} r_{i} G_{(8)} r_{i} \Delta T^{a}\right| \psi_{2}\right\rangle
$$

Note that, if $\psi_{1}=\psi_{2}=\psi, \alpha^{(\psi \psi)}$ is positive.

\section{References}

[1] E. S. Swanson, The New heavy mesons: A Status report, Phys.Rept. 429 (2006) 243-305. arXiv:hep-ph/0601110, doi:10.1016/j . physrep.2006.04.003.

[2] N. Drenska, R. Faccini, F. Piccinini, A. Polosa, F. Renga, C. Sabelli, New Hadronic Spectroscopy, Riv.Nuovo Cim. 033 (2010) $633-712$. arXiv: 1006.2741, doi:10.1393/ncr/i2010-10059-8.

[3] N. Brambilla, et al., QCD and Strongly Coupled Gauge Theories: Challenges and Perspectives, Eur.Phys.J. C74 (10) (2014) 2981. arXiv: 1404.3723, doi:10.1140/epjc/s10052-014-2981-5.

[4] A. Esposito, A. L. Guerrieri, F. Piccinini, A. Pilloni, A. D. Polosa, Four-Quark Hadrons: an Updated Review, Int.J.Mod.Phys. A30 (04n05) (2014) 1530002. arXiv: 1411.5997, doi:10.1142/S0217751X15300021. 
[5] R. F. Lebed, R. E. Mitchell, E. S. Swanson, Heavy-Quark QCD Exotica (2016). arXiv: 1610.04528.

[6] H.-X. Chen, W. Chen, X. Liu, S.-L. Zhu, The hidden-charm pentaquark and tetraquark states, Phys.Rept. 639 (2016) 1-121. arXiv: 1601.02092, doi:10.1016/j.physrep.2016.05.004.

[7] L. Maiani, F. Piccinini, A. D. Polosa, V. Riquer, Diquark-antidiquarks with hidden or open charm and the nature of $X(3872)$, Phys.Rev. D71 (2005) 014028. arXiv:hep-ph/0412098, doi:10.1103/PhysRevD.71.014028.

[8] L. Maiani, F. Piccinini, A. Polosa, V. Riquer, The Z(4430) and a New Paradigm for Spin Interactions in Tetraquarks, Phys.Rev. D89 (2014) 114010. arXiv:1405.1551, doi:10.1103/PhysRevD.89.114010.

[9] L. Maiani, A. D. Polosa, V. Riquer, Tetraquarks in the $1 / N$ expansion and meson-meson resonances, JHEP 06 (2016) 160. arXiv:1605. 04839, doi: 10.1007/JHEP06 (2016) 160.

[10] M. Papinutto, F. Piccinini, A. Pilloni, A. D. Polosa, N. Tantalo, A Tentative Description of $Z_{c, b}$ States in Terms of Metastable Feshbach Resonances, in: Proceedings, 6th International Workshop on Charm Physics (Charm 2013), 2013. arXiv:1311.7374. URL http://inspirehep.net/record/1266792/files/arXiv:1311.7374.pdf

[11] A. Esposito, A. Pilloni, A. D. Polosa, Hybridized Tetraquarks, Phys.Lett. B758 (2016) 292-295. arXiv:1603.07667, doi:10.1016/j . physletb.2016.05.028.

[12] L. D. Landau, E. M. Lifshitz, Quantum Mechanichs, Vol. 3, §133 (2003).

[13] S. Weinberg, Lectures on Quantum Mechanics (Second Edition), \$8.8 (2015).

[14] S. Weinberg, Evidence That the Deuteron Is Not an Elementary Particle, Phys.Rev. 137 (1965) B672-B678. doi : 10.1103/PhysRev.137. B672.

[15] S. Weinberg, The Quantum Theory of Fields. Vol. 1: Foundations, §3.6 (2005).

[16] L. D. Landau, Soviet Phys.-JETP 39 (1960) 1856.

[17] A. Polosa, Constraints from precision measurements on the hadron-molecule interpretation of $X, Y, Z$ resonances, Phys.Lett. B746 (2015) 248-250. arXiv:1505.03083, doi:10.1016/j.physletb.2015.05.017.

[18] M. Voloshin, Interference and binding effects in decays of possible molecular component of X(3872), Phys.Lett. B579 (2004) 316-320. arXiv:hep-ph/0309307, doi:10.1016/j.physletb.2003.11.014.

[19] E. Braaten, M. Kusunoki, Low-energy universality and the new charmonium resonance at $3870 \mathrm{MeV}$, Phys.Rev. D69 (2004) 074005 . arXiv:hep-ph/0311147, doi:10.1103/PhysRevD.69.074005.

[20] A. Esposito, F. Piccinini, A. Pilloni, A. Polosa, A Mechanism for Hadron Molecule Production in $p \bar{p}(p)$ Collisions, J.Mod.Phys. 4 (2013) 1569-1573. arXiv:1305.0527, doi:10.4236/jmp.2013.412193.

[21] E. Braaten, H. W. Hammer, Universality in few-body systems with large scattering length, Phys.Rept. 428 (2006) 259-390. arXiv: cond-mat/0410417, doi:10.1016/j.physrep.2006.03.001.

[22] A. Leggett, Quantum Liquids: Bose Condensation and Cooper Pairing in Condensed-matter Systems, §4A, (4.A.28), Oxford Graduate Texts, OUP Oxford, 2006

URL https://books .google.com/books?id=kywSDAAAQBAJ

[23] C. Pethick, H. Smith, Bose-Einstein Condensation in Dilute Gases (Second Edition), §5.4.2, Cambridge University Press, 2002. URL https://books.google.com/books?id=iBk0G3_5iIQC

[24] W. Buchmuller, S. H. H. Tye, Quarkonia and Quantum Chromodynamics, Phys.Rev. D24 (1981) 132. doi:10.1103/PhysRevD.24.132.

[25] S. Godfrey, N. Isgur, Mesons in a Relativized Quark Model with Chromodynamics, Phys.Rev. D32 (1985) 189-231. doi:10.1103/ PhysRevD.32.189.

[26] E. Braaten, M. Lu, Line shapes of the X(3872), Phys.Rev. D76 (2007) 094028. arXiv: 0709. 2697, doi : 10.1103/PhysRevD.76.094028.

[27] S.-K. Choi, et al. (Belle collaboration), Observation of a new narrow charmonium state in exclusive $B^{ \pm} \rightarrow K^{ \pm} \pi^{+} \pi^{-} J \psi$ decays, Phys.Rev.Lett. 91 (2003) 262001. arXiv: hep-ex/0309032, doi:10.1103/PhysRevLett.91.262001.

[28] A. Esposito, A. L. Guerrieri, L. Maiani, F. Piccinini, A. Pilloni, A. D. Polosa, V. Riquer, Observation of light nuclei at ALICE and the X(3872) conundrum, Phys.Rev. D92 (3) (2015) 034028. arXiv:1508.00295, doi:10.1103/PhysRevD.92.034028.

[29] A. Tomaradze, S. Dobbs, T. Xiao, K. K. Seth, Precision Measurement of the Mass of the $D^{* 0}$ Meson and the Binding Energy of the $X(3872)$ Meson as a $D^{0} \overline{D^{* 0}}$ Molecule, Phys.Rev. D91 (1) (2015) 011102. arXiv:1501.01658, doi : 10.1103/PhysRevD.91.011102.

[30] A. Guerrieri, F. Piccinini, A. Pilloni, A. Polosa, Production of Tetraquarks at the LHC, Phys.Rev. D90 (2014) 034003. arXiv: 1405.7929, doi:10.1103/PhysRevD.90.034003.

[31] C. Bignamini, B. Grinstein, F. Piccinini, A. Polosa, C. Sabelli, Is the $X(3872)$ Production Cross Section at Tevatron Compatible with a Hadron Molecule Interpretation?, Phys.Rev.Lett. 103 (2009) 162001. arXiv:0906.0882, doi:10.1103/PhysRevLett.103.162001.

[32] C. Bignamini, B. Grinstein, F. Piccinini, A. Polosa, V. Riquer, et al., More loosely bound hadron molecules at CDF?, Phys.Lett. B684 (2010) 228-230. arXiv:0912.5064, doi:10.1016/j.physletb.2010.01.037.

[33] S. J. Brodsky, R. F. Lebed, QCD dynamics of tetraquark production, Phys.Rev. D91 (2015) 114025. arXiv: 1505.00803, doi:10.1103/ PhysRevD.91.114025.

[34] F.-K. Guo, U.-G. Meißner, W. Wang, On the constituent counting rules for hard exclusive processes involving multiquark states (2016) arXiv: 1607.04020

[35] M. B. Voloshin, Constituent counting rule for exclusive production of heavy quarkoniumlike exotic resonance and a light hadron, Phys.Rev. D94 (2016) 074042. arXiv: 1608.00238, doi:10.1103/PhysRevD.94.074042.

[36] P. Artoisenet, E. Braaten, Production of the X(3872) at the Tevatron and the LHC, Phys.Rev. D81 (2010) 114018. arXiv:0911.2016, doi: 10.1103/PhysRevD.81.114018.

[37] P. Artoisenet, E. Braaten, Estimating the Production Rate of Loosely-bound Hadronic Molecules using Event Generators, Phys.Rev. D83 (2011) 014019. arXiv: 1007.2868, doi:10.1103/PhysRevD.83.014019.

[38] F.-K. Guo, U.-G. Meißner, W. Wang, Production of charged heavy quarkonium-like states at the LHC and the Tevatron, Commun.Theor.Phys. 61 (2014) 354-358. arXiv: 1308.0193, doi:10.1088/0253-6102/61/3/14.

[39] F.-K. Guo, U.-G. Meißner, W. Wang, Z. Yang, Production of the bottom analogs and the spin partner of the $X(3872)$ at hadron colliders, 
Eur.Phys.J. C74 (9) (2014) 3063. arXiv: 1402.6236, doi:10.1140/epjc/s10052-014-3063-4.

[40] M. Butenschoen, Z.-G. He, B. A. Kniehl, NLO NRQCD disfavors the interpretation of $X(3872)$ as $\chi_{c 1}(2 P)$, Phys.Rev. D88 (2013) 011501 . arXiv:1303.6524, doi:10.1103/PhysRevD.88.011501.

[41] C. Meng, H. Han, K.-T. Chao, X(3872) and its production at hadron colliders (2013). arXiv: 1304.6710.

[42] G. T. Bodwin, E. Braaten, G. P. Lepage, Rigorous QCD analysis of inclusive annihilation and production of heavy quarkonium, Phys.Rev. D51 (1995) 1125-1171, [Erratum: Phys.Rev.D55,5853(1997)]. arXiv:hep-ph/9407339, doi:10.1103/PhysRevD.55.5853,10. 1103/PhysRevD.51.1125.

[43] Y.-Q. Ma, K. Wang, K.-T. Chao, $J / \psi\left(\psi^{\prime}\right)$ production at the Tevatron and LHC at $O\left(\alpha_{s}^{4} v^{4}\right)$ in nonrelativistic QCD, Phys.Rev.Lett. 106 (2011) 042002. arXiv:1009.3655, doi:10.1103/PhysRevLett.106.042002.

[44] Y.-Q. Ma, K. Wang, K.-T. Chao, QCD radiative corrections to $\chi_{c J}$ production at hadron colliders, Phys.Rev. D83 (2011) 111503 . arXiv: 1002.3987, doi:10.1103/PhysRevD.83.111503.

[45] S. Chatrchyan, et al. (CMS collaboration), Measurement of the $X(3872)$ production cross section via decays to $J \psi \pi \pi$ in $p p$ collisions at $\sqrt{s}=7$ TeV, JHEP 1304 (2013) 154. arXiv: 1302.3968, doi : 10.1007/JHEP04(2013) 154.

[46] D. Acosta, et al. (CDF collaboration), Observation of the narrow state $X(3872) \rightarrow J / \psi \pi^{+} \pi^{-}$in $\bar{p} p$ collisions at $\sqrt{s}=1.96$ TeV, Phys. Rev.Lett. 93 (2004) 072001. arXiv: hep-ex/0312021, doi:10.1103/PhysRevLett.93.072001.

[47] R. Aaij, et al. (LHCb collaboration), Observation of X(3872) production in $p p$ collisions at $\sqrt{s}=7 \mathrm{TeV}$, Eur.Phys.J. C72 (2012) 1972 . arXiv:1112.5310, doi:10.1140/epjc/s10052-012-1972-7.

[48] A. Bondar, A. Garmash, A. Milstein, R. Mizuk, M. Voloshin, Heavy quark spin structure in $Z_{b}$ resonances, Phys.Rev. D84 (2011) 054010. arXiv:1105.4473, doi:10.1103/PhysRevD.84.054010.

[49] M. B. Voloshin, Heavy quark spin selection rule and the properties of the X(3872), Phys.Lett. B604 (2004) 69-73. arXiv: hep-ph/0408321, doi: 10.1016/j.physletb. 2004.11.003.

[50] F.-K. Guo, C. Hanhart, U.-G. Meißner, Implications of heavy quark spin symmetry on heavy meson hadronic molecules, Phys.Rev.Lett. 102 (2009) 242004. arXiv:0904.3338, doi:10.1103/PhysRevLett.102.242004.

[51] V. Baru, J. Haidenbauer, C. Hanhart, Yu. Kalashnikova, A. E. Kudryavtsev, Evidence that the a(0)(980) and $\mathrm{f}(0)(980)$ are not elementary particles, Phys.Lett. B586 (2004) 53-61. arXiv:hep-ph/0308129, doi:10.1016/j.physletb.2004.01.088.

[52] S. Fleming, M. Kusunoki, T. Mehen, U. van Kolck, Pion interactions in the X(3872), Phys.Rev. D76 (2007) 034006. arXiv:hep-ph/ 0703168, doi:10.1103/PhysRevD.76.034006.

[53] M. Cleven, F.-K. Guo, C. Hanhart, U.-G. Meißner, Bound state nature of the exotic $Z_{b}$ states, Eur.Phys.J. A47 (2011) 120. arXiv: 1107. 0254, doi:10.1140/epja/i2011-11120-6.

[54] M. Cleven, Q. Wang, F.-K. Guo, C. Hanhart, U.-G. Meißner, et al., Confirming the molecular nature of the $Z_{b}(10610)$ and the $Z_{b}(10650)$, Phys.Rev. D87 (7) (2013) 074006. arXiv:1301.6461, doi:10.1103/PhysRevD.87.074006.

[55] Q. Wang, C. Hanhart, Q. Zhao, Decoding the riddle of $Y(4260)$ and $Z_{c}$ (3900), Phys.Rev.Lett. 111 (13) (2013) 132003. arXiv: 1303.6355, doi:10.1103/PhysRevLett.111.132003.

[56] F.-K. Guo, C. Hanhart, U.-G. Meißner, Q. Wang, Q. Zhao, Production of the X(3872) in charmonia radiative decays, Phys.Lett. B725 (2013) 127-133. arXiv:1306.3096, doi:10.1016/j.physletb.2013.06.053.

[57] A. Esposito, A. Guerrieri, A. Pilloni, Probing the nature of $Z_{-} c^{\left({ }^{\prime}\right)}$ states via the $\eta_{c} \rho$ decay, Phys.Lett. B746 (2015) 194-201. arXiv: 1409.3551, doi:10.1016/j.physletb.2015.04.057.

[58] T. Mehen, Hadronic loops versus factorization in effective field theory calculations of $X(3872) \rightarrow \chi_{c J} \pi^{0}$, Phys.Rev. D92 (3) (2015) 034019 . arXiv: 1503.02719, doi:10.1103/PhysRevD.92.034019.

[59] R. Casalbuoni, A. Deandrea, N. Di Bartolomeo, R. Gatto, F. Feruglio, et al., Phenomenology of heavy meson chiral Lagrangians, Phys.Rept. 281 (1997) 145-238. arXiv: hep-ph/9605342, doi:10.1016/S0370-1573(96)00027-0.

[60] S. Weinberg, Elementary particle theory of composite particles, Phys.Rev. 130 (1963) 776-783. doi:10.1103/PhysRev.130.776.

[61] N. A. Tornqvist, Possible large deuteron-like meson meson states bound by pions, Phys.Rev.Lett. 67 (1991) 556-559. doi:10.1103/ PhysRevLett.67.556.

[62] N. A. Tornqvist, From the deuteron to deusons, an analysis of deuteron-like meson meson bound states, Z.Phys. C61 (1994) 525-537. arXiv:hep-ph/9310247, doi:10.1007/BF01413192.

[63] M. Voloshin, L. Okun, Hadron Molecules and Charmonium Atom, JETP Lett. 23 (1976) 333-336.

[64] A. De Rujula, H. Georgi, S. L. Glashow, Molecular Charmonium: A New Spectroscopy?, Phys.Rev.Lett. 38 (1977) 317. doi :10.1103/ PhysRevLett.38.317.

[65] N. A. Tornqvist, Isospin breaking of the narrow charmonium state of Belle at $3872 \mathrm{MeV}$ as a deuson, Phys.Lett. B590 (2004) 209-215, this report supersedes the unpublished reminder hep-ph/0308277. arXiv:hep-ph/0402237, doi:10.1016/j.physletb.2004.03.077.

[66] M. Cleven, F.-K. Guo, C. Hanhart, Q. Wang, Q. Zhao, Employing spin symmetry to disentangle different models for the XYZ states, Phys.Rev. D92 (1) (2015) 014005. arXiv: 1505.01771, doi : 10.1103/PhysRevD.92.014005.

[67] A. V. Manohar, M. B. Wise, Exotic Q Q anti-q anti-q states in QCD, Nucl.Phys. B399 (1993) 17-33. arXiv:hep-ph/9212236, doi: 10.1016/0550-3213(93) 90614-U.

[68] T. E. O. Ericson, G. Karl, Strength of pion exchange in hadronic molecules, Phys.Lett. B309 (1993) 426-430. doi:10.1016/ 0370-2693 (93) 90957-J.

[69] Y. Kalashnikova, A. Nefediev, One-pion exchange in the $X(3872)$ revisited, Pisma Zh.Eksp.Teor.Fiz. 97 (2013) 76-81. arXiv : 1212.2004, doi : 10.1134/S0021364013020070.

[70] Y.-R. Liu, X. Liu, W.-Z. Deng, S.-L. Zhu, Is X(3872) Really a Molecular State?, Eur.Phys.J. C56 (2008) 63-73. arXiv:0801.3540, doi:10.1140/epjc/s10052-008-0640-4.

[71] M. Suzuki, The X(3872) boson: Molecule or charmonium, Phys.Rev. D72 (2005) 114013. arXiv:hep-ph/0508258, doi:10.1103/ PhysRevD.72.114013.

[72] V. Baru, A. A. Filin, C. Hanhart, Yu. S. Kalashnikova, A. E. Kudryavtsev, A. V. Nefediev, Three-body $D \bar{D} \pi$ dynamics for the X(3872), 
Phys.Rev. D84 (2011) 074029. arXiv:1108.5644, doi:10.1103/PhysRevD.84.074029.

[73] V. Baru, E. Epelbaum, A. A. Filin, C. Hanhart, U.-G. Meißner, A. V. Nefediev, Quark mass dependence of the X(3872) binding energy, Phys.Lett. B726 (2013) 537-543. arXiv:1306.4108, doi:10.1016/j . physletb. 2013.08.073.

[74] T. Barnes, E. Swanson, A Diagrammatic approach to meson meson scattering in the nonrelativistic quark potential model, Phys.Rev. D46 (1992) 131-159. doi:10.1103/PhysRevD.46.131.

[75] E. S. Swanson, Short range structure in the X(3872), Phys.Lett. B588 (2004) 189-195. arXiv:hep-ph/0311229, doi:10.1016/j . physletb.2004.03.033.

[76] R. L. Jaffe, F. Wilczek, Diquarks and exotic spectroscopy, Phys.Rev.Lett. 91 (2003) 232003. arXiv:hep-ph/0307341, doi:10.1103/ PhysRevLett.91.232003.

[77] N. Cardoso, M. Cardoso, P. Bicudo, Colour Fields Computed in SU(3) Lattice QCD for the Static Tetraquark System, Phys.Rev. D84 (2011) 054508. arXiv:1107.1355, doi:10.1103/PhysRevD.84.054508.

[78] G. Rossi, G. Veneziano, A Possible Description of Baryon Dynamics in Dual and Gauge Theories, Nucl.Phys. B123 (1977) 507. doi: 10.1016/0550-3213(77) 90178-X.

[79] L. Montanet, G. Rossi, G. Veneziano, Baryonium Physics, Phys.Rept. 63 (1980) 149-222.

[80] G. Rossi, G. Veneziano, Electromagnetic Mixing of Narrow Baryonium States, Phys.Lett. B70 (1977) 255. doi : 10. 1016/0370-2693 (77) 90533-0.

[81] G. Cotugno, R. Faccini, A. Polosa, C. Sabelli, Charmed Baryonium, Phys.Rev.Lett. 104 (2010) 132005. arXiv:0911.2178, doi:10. 1103/PhysRevLett.104.132005.

[82] G. Rossi, G. Veneziano, The string-junction picture of multiquark states: an update, JHEP 06 (2016) 041. arXiv:1603.05830, doi: 10.1007/JHEP06 (2016) 041.

[83] L. Maiani, V. Riquer, F. Piccinini, A. Polosa, Four quark interpretation of $Y$ (4260), Phys.Rev. D72 (2005) 031502. arXiv: hep-ph/0507062, doi:10.1103/PhysRevD.72.031502.

[84] N. V. Drenska, R. Faccini, A. D. Polosa, Higher Tetraquark Particles, Phys.Lett. B669 (2008) 160-166. arXiv: 0807.0593, doi:10.1016/ j.physletb.2008.09.038.

[85] N. V. Drenska, R. Faccini, A. D. Polosa, Exotic Hadrons with Hidden Charm and Strangeness, Phys.Rev. D79 (2009) 077502. arXiv: 0902.2803, doi:10.1103/PhysRevD.79.077502.

[86] L. Maiani, A. D. Polosa, V. Riquer, Indications of a Four-Quark Structure for the X(3872) and X(3876) Particles from Recent Belle and BABAR Data, Phys.Rev.Lett. 99 (2007) 182003. arXiv:0707.3354, doi:10.1103/PhysRevLett.99.182003.

[87] L. Maiani, V. Riquer, R. Faccini, F. Piccinini, A. Pilloni, et al., $J^{P G}=1^{++}$Charged Resonance in the $Y(4260) \rightarrow \pi^{+} \pi^{-} J / \psi$ Decay?, Phys.Rev. D87 (11) (2013) 111102. arXiv: 1303.6857, doi:10.1103/PhysRevD.87.111102.

[88] S. J. Brodsky, D. S. Hwang, R. F. Lebed, A New Picture for the Formation and Decay of the Exotic XYZ Mesons, Phys.Rev.Lett. 113 (2014) 112001. arXiv:1406.7281, doi:10.1103/PhysRevLett.113.112001.

[89] S. H. Blitz, R. F. Lebed, Tetraquark Cusp Effects from Diquark Pair Production, Phys.Rev. D91 (9) (2015) 094025. arXiv: 1503.04802, doi:10.1103/PhysRevD.91.094025.

[90] N. Barnea, J. Vijande, A. Valcarce, Four-quark spectroscopy within the hyperspherical formalism, Phys.Rev. D73 (2006) 054004. arXiv : hep-ph/0604010, doi:10.1103/PhysRevD.73.054004.

[91] J. Vijande, E. Weissman, N. Barnea, A. Valcarce, Do $c \bar{c} n \bar{n}$ bound states exist?, Phys.Rev. D76 (2007) 094022. arXiv: 0708. 3285, doi: 10.1103/PhysRevD.76.094022.

[92] J. Vijande, E. Weissman, A. Valcarce, N. Barnea, Are there compact heavy four-quark bound states?, Phys.Rev. D76 (2007) 094027. arXiv : 0710.2516, doi:10.1103/PhysRevD.76.094027.

[93] T. Fernandez-Carames, A. Valcarce, J. Vijande, Charmonium spectroscopy above thresholds, Phys.Rev.Lett. 103 (2009) 222001. arXiv: 1001.4506, doi:10.1103/PhysRevLett.103.222001.

[94] J. Vijande, A. Valcarce, J.-M. Richard, Adiabaticity and color mixing in tetraquark spectroscopy, Phys.Rev. D87 (2013) 034040. arXiv : 1301.6212, doi:10.1103/PhysRevD.87.034040.

[95] F. Buccella, H. Hogaasen, J.-M. Richard, P. Sorba, Chromomagnetism, flavour symmetry breaking and S-wave tetraquarks, Eur.Phys.J. C49 (2007) 743-754. arXiv: hep-ph/0608001, doi:10.1140/epjc/s10052-006-0142-1.

[96] M. Abud, F. Buccella, F. Tramontano, Hints for the existence of hexaquark states in the baryon-antibaryon sector, Phys.Rev. D81 (2010) 074018. arXiv:0912.4299, doi:10.1103/PhysRevD.81.074018.

[97] J. Ferretti, G. Galatà, E. Santopinto, Interpretation of the $X(3872)$ as a charmonium state plus an extra component due to the coupling to the meson-meson continuum, Phys.Rev. C88 (1) (2013) 015207. arXiv: 1302.6857, doi:10.1103/PhysRevC. 88.015207.

[98] E. Santopinto, A. Giachino, Compact pentaquark structures (2016). arXiv: 1604.03769.

[99] J.-M. Richard, Exotic hadrons: review and perspectives, Few Body Syst. 57 (12) (2016) 1185-1212. arXiv: 1606.08593, doi:10.1007/ s00601-016-1159-0.

[100] G. Eichmann, C. S. Fischer, W. Heupel, The light scalar mesons as tetraquarks, Phys.Lett. B753 (2016) 282-287. arXiv:1508.07178, doi: 10.1016/j.physletb.2015.12.036.

[101] K. Rajagopal, F. Wilczek, The Condensed matter physics of QCD (2000). arXiv: hep-ph/0011333.

[102] F. Wilczek, Diquarks as inspiration and as objects, in: From fields to strings: Circumnavigating theoretical physics. Ian Kogan memorial collection (3 volume set), 2004, pp. 322-338, [,77(2004)]. arXiv: hep-ph/0409168.

[103] R. Jaffe, Exotica, Phys.Rept. 409 (2005) 1-45. arXiv: hep-ph/0409065, doi :10.1016/j .physrep. 2004.11.005.

[104] R. D. Pisarski, V. V. Skokov, How tetraquarks can generate a second chiral phase transition, Phys.Rev. D94 (5) (2016) 054008. arXiv: 1606.04111, doi:10.1103/PhysRevD.94.054008.

[105] L. Maiani, F. Piccinini, A. Polosa, V. Riquer, A New look at scalar mesons, Phys.Rev.Lett. 93 (2004) 212002. arXiv:hep-ph/0407017, doi:10.1103/PhysRevLett.93.212002.

[106] G. 't Hooft, G. Isidori, L. Maiani, A. Polosa, V. Riquer, A Theory of Scalar Mesons, Phys.Lett. B662 (2008) 424-430. arXiv : 0801.2288, 
doi:10.1016/j.physletb.2008.03.036.

[107] S. L. Olsen, XYZ Meson Spectroscopy, in: Proceedings, 53rd International Winter Meeting on Nuclear Physics (Bormio 2015): Bormio, Italy, January 26-30, 2015, 2015. arXiv: 1511.01589.

URL http://inspirehep.net/record/1402960/files/arXiv:1511.01589.pdf

[108] M. Ablikim, et al. (BES III collaboration), Precise measurement of the $e^{+} e^{-} \rightarrow \pi^{+} \pi^{-} J / \psi$ cross section at center-of-mass energies from 3.77 to $4.60 \mathrm{GeV}$ (2016). arXiv : 1611.01317.

[109] M. Ablikim, et al. (BES III collaboration), Observation of two resonant structures in $e^{+} e^{-} \rightarrow \pi^{+} \pi^{-} h_{c}$ (2016). arXiv:1610.07044.

[110] M. Ablikim, et al. (BESIII collaboration), Observation of $e^{+} e^{-} \rightarrow \gamma X(3872)$ at BESIII, Phys.Rev.Lett. 112 (9) (2014) 092001. arXiv: 1310.4101, doi:10.1103/PhysRevLett.112.092001.

[111] R. Faccini, G. Filaci, A. Guerrieri, A. Pilloni, A. Polosa, Note on the newly observed Y(4220) resonance, Phys.Rev. D91 (11) (2015) 117501. arXiv:1412.7196, doi:10.1103/PhysRevD.91.117501.

[112] C.-Z. Yuan, Evidence for resonant structures in $e^{+} e^{-} \rightarrow \pi^{+} \pi^{-} h_{c}$, Chin.Phys. C38 (2014) 043001. arXiv:1312.6399, doi:10.1088/ $1674-1137 / 38 / 4 / 043001$

[113] C.-Z. Yuan, Recent progress on the study of the charmoniumlike states, Int.J.Mod.Phys. A29 (2014) 1430046. arXiv: 1404.7768, doi: 10.1142/S0217751X14300464.

[114] R. Aaij, et al. (LHCb collaboration), Observation of $J / \psi p$ resonances consistent with pentaquark states in $\Lambda \_b^{0} \rightarrow J / \psi K^{-} p$ decays, Phys.Rev.Lett. 115 (2015) 072001. arXiv: 1507.03414, doi:10.1103/PhysRevLett.115.072001.

[115] C. Alexandrou, P. de Forcrand, B. Lucini, Evidence for diquarks in lattice QCD, Phys.Rev.Lett. 97 (2006) 222002. arXiv:hep-1at/ 0609004, doi:10.1103/PhysRevLett.97.222002.

[116] A. De Rujula, H. Georgi, S. L. Glashow, Hadron Masses in a Gauge Theory, Phys.Rev. D12 (1975) 147-162. doi:10.1103/PhysRevD. 12.147.

[117] L. Maiani, A. D. Polosa, V. Riquer, The New Pentaquarks in the Diquark Model, Phys.Lett. B749 (2015) 289-291. arXiv:1507.04980, doi:10.1016/j.physletb.2015.08.008.

[118] L. Maiani, A. D. Polosa, V. Riquer, From pentaquarks to dibaryons in $\Lambda_{b}(5620)$ decays, Phys.Lett. B750 (2015) 37-38. arXiv : 1508.04459, doi:10.1016/j.physletb.2015.08.049.

[119] A. Ali, I. Ahmed, M. J. Aslam, A. Rehman, Heavy quark symmetry and weak decays of the $b$-baryons in pentaquarks with a $c \bar{c}$ component, Phys.Rev. D94 (5) (2016) 054001. arXiv:1607.00987, doi : 10.1103/PhysRevD.94.054001.

[120] S. Weinberg, Tetraquark Mesons in Large $N$ Quantum Chromodynamics, Phys.Rev.Lett. 110 (26) (2013) 261601. arXiv:1303.0342, doi:10.1103/PhysRevLett.110.261601.

[121] M. Knecht, S. Peris, Narrow Tetraquarks at Large N, Phys.Rev. D88 (2013) 036016. arXiv:1307.1273, doi:10.1103/PhysRevD.88. 036016.

[122] T. D. Cohen, R. F. Lebed, Are There Tetraquarks at Large $N_{c}$ in QCD(F)?, Phys.Rev. D90 (2014) 016001. arXiv:1403.8090, doi: 10.1103/PhysRevD.90.016001.

[123] S. Coleman, Aspects of Symmetry, Cambridge University Press, Cambridge, England, 1985.

[124] E. Witten, Baryons in the 1/N Expansion, Nucl.Phys. B160 (1979) 57. doi:10.1016/0550-3213(79)90232-3.

[125] R. F. Lebed, Large-N Structure of Tetraquark Mesons, Phys.Rev. D88 (2013) 057901. arXiv: 1308. 2657, doi:10.1103/PhysRevD.88. 057901.

[126] T. D. Cohen, R. F. Lebed, Quantum-Number Exotic Tetraquarks at Large $N_{c}$ in QCD(AS), Phys.Rev. D89 (2014) 054018. arXiv: 1401. 1815, doi:10.1103/PhysRevD.89.054018.

[127] T. Cohen, F. J. Llanes-Estrada, J. R. Peláez, J. Ruiz de Elvira, Nonordinary light meson couplings and the $1 / N_{c}$ expansion, Phys.Rev. D90 (3) (2014) 036003. arXiv: 1405.4831, doi:10.1103/PhysRevD.90.036003.

[128] G. 't Hooft, A Two-Dimensional Model for Mesons, Nucl.Phys. B75 (1974) 461. doi:10.1016/0550-3213(74) 90088-1.

[129] M. Bochicchio, S. P. Muscinelli, Ultraviolet asymptotics of glueball propagators, JHEP 08 (2013) 064. arXiv : 1304.6409, doi : 10.1007/ JHEP08 (2013) 064

[130] E. Corrigan, P. Ramond, A Note on the Quark Content of Large Color Groups, Phys.Lett. B87 (1979) 73. doi:10.1016/0370-2693 (79) 90022-4.

[131] R. L. Jaffe, Ordinary and extraordinary hadrons, AIP Conf. Proc. 964 (2007) 1-13, [Prog. Theor. Phys. Suppl.168,127(2007)]. arXiv: hep-ph/0701038, doi:10.1063/1.2823850.

[132] C. Hanhart, J. R. Peláez, G. Rios, Remarks on pole trajectories for resonances, Phys.Lett. B739 (2014) 375-382. arXiv:1407.7452, doi:10.1016/j.physletb.2014.11.011.

[133] V. M. Abazov, et al. (D $\varnothing$ collaboration), Evidence for a $B_{s}^{0} \pi^{ \pm}$state, Phys.Rev.Lett. 117 (2) (2016) 022003. arXiv:1602.07588, doi : 10.1103/PhysRevLett.117.022003.

[134] S. Chatrchyan, et al. (CMS collaboration), Observation of a peaking structure in the $J / \psi \phi$ mass spectrum from $B^{ \pm} \rightarrow J / \psi \phi K^{ \pm}$decays, Phys.Lett. B734 (2014) 261. arXiv:1309.6920, doi:10.1016/j.physletb.2014.05.055.

[135] R. Aaij, et al. (LHCb collaboration), Amplitude analysis of $B^{+} \rightarrow J / \psi \phi K^{+}$decays (2016). arXiv: 1606.07898.

[136] R. Aaij, et al. (LHCb collaboration), Observation of $J / \psi \phi$ structures consistent with exotic states from amplitude analysis of $B^{+} \rightarrow J / \psi \phi K^{+}$ decays (2016). arXiv: 1606.07895.

[137] C. Patrignani, et al. (Particle Data Group collaboration), Review of Particle Physics, Chin.Phys. C40 (10) (2016) 100001. doi : 10.1088/ 1674-1137/40/10/100001.

[138] E. Braaten, M. Kusunoki, Exclusive production of the X(3872) in B meson decay, Phys.Rev. D71 (2005) 074005. arXiv: hep-ph/0412268, doi: 10.1103/PhysRevD.71.074005.

[139] R. F. Lebed, A. D. Polosa, $\chi_{c 0}$ (3915) As the Lightest $c \bar{c} s \bar{s}$ State, Phys.Rev. D93 (9) (2016) 094024. arXiv: 1602.08421, doi:10.1103/ PhysRevD.93.094024.

[140] L. Maiani, A. D. Polosa, V. Riquer, Interpretation of Axial Resonances in $J / \psi \phi$ at LHCb, Phys.Rev. D94 (5) (2016) 054026. arXiv: 
1607.02405, doi:10.1103/PhysRevD.94.054026.

[141] L. Maiani, A. D. Polosa, V. Riquer, Interpretation of Axial Resonances in $J / \psi \phi$ at LHCb, Phys.Rev. D94 (5) (2016) 054026. arXiv: 1607.02405, doi:10.1103/PhysRevD.94.054026.

[142] J. Wu, Y.-R. Liu, K. Chen, X. Liu, S.-L. Zhu, The $X(4140), X(4270), X(4500)$ and $X(4700)$ and their $c s \bar{c} \bar{s}$ tetraquark partners (2016). arXiv: 1608.07900 .

[143] M. Albaladejo, F.-K. Guo, C. Hidalgo-Duque, J. Nieves, $Z_{c}(3900)$ : What has been really seen?, Phys.Lett. B755 (2016) 337-342. arXiv : 1512.03638, doi:10.1016/j.physletb.2016.02.025.

[144] F. K. Guo, C. Hanhart, Yu. S. Kalashnikova, P. Matuschek, R. V. Mizuk, A. V. Nefediev, Q. Wang, J. L. Wynen, Interplay of quark and meson degrees of freedom in near-threshold states: A practical parametrization for line shapes, Phys.Rev. D93 (7) (2016) 074031. arXiv: 1602.00940, doi:10.1103/PhysRevD.93.074031.

[145] S. Dubynskiy, M. Voloshin, Hadro-Charmonium, Phys.Lett. B666 (2008) 344-346. arXiv:0803.2224, doi:10.1016/j.physletb. 2008.07 .086$.

[146] S. Dubynskiy, A. Gorsky, M. Voloshin, Holographic Hadro-Quarkonium, Phys.Lett. B671 (2009) 82-86. arXiv:0804.2244, doi:10. $1016 / j$.physletb.2008.11.040.

[147] X. Li, M. Voloshin, Y(4260) and Y(4360) as mixed hadrocharmonium, Mod.Phys.Lett. A29 (2014) 1450060. arXiv:1309.1681, doi: 10.1142/S0217732314500606.

[148] Q. Wang, M. Cleven, F.-K. Guo, C. Hanhart, U.-G. Meißner, X.-G. Wu, Q. Zhao, Y(4260): hadronic molecule versus hadro-charmonium interpretation, Phys.Rev. D89 (3) (2014) 034001. arXiv: 1309.4303, doi : 10.1103/PhysRevD.89.034001.

[149] M. Alberti, G. S. Bali, S. Collins, F. Knechtli, G. Moir, W. Sldner, Hadro-quarkonium from Lattice QCD (2016). arXiv : 1608.06537.

[150] K. Gottfried, Hadronic Transitions Between Quark - anti-Quark Bound States, Phys.Rev.Lett. 40 (1978) 598. doi : 10.1103/PhysRevLett. 40.598.

[151] M. B. Voloshin, On Dynamics of Heavy Quarks in Nonperturbative QCD Vacuum, Nucl.Phys. B154 (1979) 365-380. doi:10.1016/ 0550-3213 (79) 90037-3.

[152] M. E. Peskin, Short Distance Analysis for Heavy Quark Systems. 1. Diagrammatics, Nucl.Phys. B156 (1979) 365-390. doi : 10.1016/ 0550-3213(79) 90199-8.

[153] G. Bhanot, M. E. Peskin, Short Distance Analysis for Heavy Quark Systems. 2. Applications, Nucl.Phys. B156 (1979) 391-416. doi : 10.1016/0550-3213(79) 90200-1.

[154] M. Voloshin, Quarkonium chromo-polarizability from the decays $J \psi(\Upsilon) \rightarrow \pi \pi l^{+} l^{-}$, Mod.Phys.Lett. A19 (2004) 665-670. arXiv: hep-ph/ 0402011, doi:10.1142/S0217732304013568.

[155] Z. Liu, et al. (Belle collaboration), Study of $e^{+} e^{-} \rightarrow \pi^{+} \pi^{-} J \psi$ and Observation of a Charged Charmoniumlike State at Belle, Phys.Rev.Lett. 110 (25) (2013) 252002. arXiv:1304.0121, doi:10.1103/PhysRevLett.110.252002.

[156] M. Ablikim, et al. (BESIII collaboration), Observation of a Charged Charmoniumlike Structure in $e^{+} e^{-} \rightarrow \pi^{+} \pi^{-} J \psi$ at $\sqrt{s}=4.26 \mathrm{GeV}$, Phys.Rev.Lett. 110 (25) (2013) 252001. arXiv: 1303.5949, doi : 10.1103/PhysRevLett.110.252001.

[157] A. Karch, E. Katz, D. T. Son, M. A. Stephanov, Linear confinement and AdS/QCD, Phys.Rev. D74 (2006) 015005. arXiv:hep-ph/ 0602229, doi:10.1103/PhysRevD.74.015005.

[158] M. B. Voloshin, $Z_{c}$ (3900) - what is inside?, Phys.Rev. D87 (9) (2013) 091501. arXiv: 1304.0380, doi : 10.1103/PhysRevD.87.091501.

[159] M. Ablikim, et al. (BESIII collaboration), Observation of a charged $\left(D \bar{D}^{*}\right)^{ \pm}$mass peak in $e^{+} e^{-} \rightarrow \pi^{+} D \bar{D}^{*}$ at $\sqrt{s}=4.26 \mathrm{GeV}$, Phys. Rev.Lett. 112 (2014) 022001. arXiv: 1310.1163, doi:10.1103/PhysRevLett.112.022001.

[160] V. Kubarovsky, M. B. Voloshin, Formation of hidden-charm pentaquarks in photon-nucleon collisions, Phys.Rev. D92 (3) (2015) 031502. arXiv: 1508.00888, doi:10.1103/PhysRevD.92.031502.

[161] M. Ablikim, et al. (BESIII collaboration), Observation of a charged charmoniumlike structure $Z_{c}(4020)$ and search for the $Z_{c}(3900)$ in $e^{+} e^{-} \rightarrow \pi^{+} \pi^{-} h_{c}$, Phys.Rev.Lett. 111 (2013) 242001. arXiv: 1309.1896, doi:10.1103/PhysRevLett.111.242001.

[162] V. Mathieu, N. Kochelev, V. Vento, The Physics of Glueballs, Int.J.Mod.Phys. E18 (2009) 1-49. arXiv:0810.4453, doi:10.1142/ S0218301309012124.

[163] G. S. Bali, K. Schilling, A. Hulsebos, A. C. Irving, C. Michael, P. W. Stephenson (UKQCD collaboration), A Comprehensive lattice study of SU(3) glueballs, Phys.Lett. B309 (1993) 378-384. arXiv: hep-lat/9304012, doi:10.1016/0370-2693(93)90948-H.

[164] C. J. Morningstar, M. J. Peardon, The Glueball spectrum from an anisotropic lattice study, Phys.Rev. D60 (1999) 034509. arXiv: hep-lat/ 9901004, doi:10.1103/PhysRevD.60.034509.

[165] H. B. Meyer, M. J. Teper, High spin glueballs from the lattice, Nucl.Phys. B658 (2003) 113-155. arXiv:hep-lat/0212026, doi: 10.1016/S0550-3213(03)00183-4.

[166] E. Gregory, A. Irving, B. Lucini, C. McNeile, A. Rago, C. Richards, E. Rinaldi, Towards the glueball spectrum from unquenched lattice QCD, JHEP 10 (2012) 170. arXiv: 1208.1858, doi:10.1007/JHEP10(2012) 170.

[167] D. Horn, J. Mandula, A Model of Mesons with Constituent Gluons, Phys.Rev. D17 (1978) 898. doi : 10.1103/PhysRevD.17.898.

[168] M. Tanimoto, Decay of an Exotic $q \bar{q} g$ Hybrid Meson, Phys.Rev. D27 (1983) 2648. doi :10.1103/PhysRevD.27.2648.

[169] M. Tanimoto, Decay Patterns of $q \bar{q} g$ Hybrid Mesons, Phys.Lett. B116 (1982) 198. doi : 10.1016/0370-2693(82) 91008-5.

[170] F. Iddir, A. Le Yaouanc, L. Oliver, O. Pene, J. Raynal, et al., $q \bar{q} g$ Hybrid and $q q \bar{q} \bar{q}$ Diquonium Interpretation of GAMS $1^{-+}$Resonance, Phys.Lett. B205 (1988) 564. doi : 10.1016/0370-2693(88)90999-9.

[171] S. Ishida, M. Oda, H. Sawazaki, K. Yamada, Is the $f_{1}(1420)$ Our First Hybrid Meson?, Prog.Theor.Phys. 82 (1989) 119. doi:10.1143/ PTP. 82.119.

[172] S. Ishida, H. Sawazaki, M. Oda, K. Yamada, Decay properties of hybrid mesons with a massive constituent gluon and search for their candidates, Phys.Rev. D47 (1993) 179-198. doi : 10.1103/PhysRevD.47.179.

[173] R. Jaffe, K. Johnson, Unconventional States of Confined Quarks and Gluons, Phys.Lett. B60 (1976) 201. doi:10.1016/0370-2693(76) 90423-8.

[174] P. Hasenfratz, R. Horgan, J. Kuti, J. Richard, The Effects of Colored Glue in the QCD Motivated Bag of Heavy Quark - anti-Quark Systems, 
Phys.Lett. B95 (1980) 299. doi : 10.1016/0370-2693(80)90491-8.

[175] M. Berwein, N. Brambilla, J. Tarrús Castellà, A. Vairo, Quarkonium Hybrids with Nonrelativistic Effective Field Theories, Phys.Rev. D92 (11) (2015) 114019. arXiv: 1510.04299, doi:10.1103/PhysRevD.92.114019.

[176] J. F. Arvis, The Exact $q \bar{q}$ Potential in Nambu String Theory, Phys.Lett. B127 (1983) 106-108. doi : 10.1016/0370-2693(83) 91640-4.

[177] K. J. Juge, J. Kuti, C. Morningstar, Fine structure of the QCD string spectrum, Phys.Rev.Lett. 90 (2003) 161601. arXiv: hep-lat/0207004, doi: $10.1103 /$ PhysRevLett.90.161601.

[178] J. Polchinski, String Theory: Volume 1, An Introduction to the Bosonic String, Cambridge Monographs on Mathematical Physics, Cambridge University Press, 1998.

URL https://books.google.com/books?id=jbM3t_usmXOC

[179] M. Lüscher, Symmetry Breaking Aspects of the Roughening Transition in Gauge Theories, Nucl.Phys. B180 (1981) 317-329. doi:10 . 1016/0550-3213(81) 90423-5.

[180] S. Necco, R. Sommer, The $N_{f}=0$ heavy quark potential from short to intermediate distances, Nucl.Phys. B622 (2002) 328-346. arXiv : hep-lat/0108008, doi:10.1016/S0550-3213(01)00582-X.

[181] E. Eichten, K. Gottfried, T. Kinoshita, K. D. Lane, T.-M. Yan, Charmonium: The Model, Phys.Rev. D17 (1978) 3090 , [Erratum: Phys.Rev.D21,313(1980)]. doi:10.1103/PhysRevD.17.3090,10.1103/PhysRevD.21.313.

[182] N. Isgur, J. E. Paton, A Flux Tube Model for Hadrons in QCD, Phys.Rev. D31 (1985) 2910. doi : 10.1103/PhysRevD.31.2910.

[183] R. Kokoski, N. Isgur, Meson Decays by Flux Tube Breaking, Phys.Rev. D35 (1987) 907. doi : 10.1103/PhysRevD.35.907.

[184] F. E. Close, P. R. Page, The Production and decay of hybrid mesons by flux tube breaking, Nucl.Phys. B443 (1995) 233-254. arXiv: hep-ph/9411301, doi:10.1016/0550-3213(95)00085-7.

[185] N. Isgur, Flux tube zero point motion, hadronic charge radii, and hybrid meson production cross-sections, Phys.Rev. D60 (1999) 114016. arXiv: hep-ph/9904494, doi:10.1103/PhysRevD.60.114016.

[186] T. Barnes, F. Close, E. Swanson, Hybrid and conventional mesons in the flux tube model: Numerical studies and their phenomenological implications, Phys.Rev. D52 (1995) 5242-5256. arXiv: hep-ph/9501405, doi : 10.1103/PhysRevD.52.5242.

[187] E. S. Swanson, A. P. Szczepaniak, Heavy hybrids with constituent gluons, Phys.Rev. D59 (1999) 014035. arXiv:hep-ph/9804219, doi:10.1103/PhysRevD.59.014035.

[188] P. Guo, A. P. Szczepaniak, G. Galata, A. Vassallo, E. Santopinto, Heavy quarkonium hybrids from Coulomb gauge QCD, Phys.Rev. D78 (2008) 056003. arXiv:0807.2721, doi:10.1103/PhysRevD.78.056003.

[189] A. P. Szczepaniak, P. Krupinski, Energy spectrum of the low-lying gluon excitations in the Coulomb gauge, Phys.Rev. D73 (2006) 116002. arXiv:hep-ph/0604098, doi:10.1103/PhysRevD.73.116002.

[190] P. Guo, T. Yépez-Martínez, A. P. Szczepaniak, Charmonium meson and hybrid radiative transitions, Phys.Rev. D89 (11) (2014) 116005. arXiv:1402.5863, doi:10.1103/PhysRevD.89.116005.

[191] N. H. Christ, T. D. Lee, Operator Ordering and Feynman Rules in Gauge Theories, Phys.Rev. D22 (1980) 939, [Phys. Scripta23,970(1981)]. doi:10.1103/PhysRevD.22.939.

[192] A. P. Szczepaniak, E. S. Swanson, Coulomb gauge QCD, confinement, and the constituent representation, Phys.Rev. D65 (2002) 025012. arXiv:hep-ph/0107078, doi:10.1103/PhysRevD.65.025012.

[193] P. Guo, A. P. Szczepaniak, G. Galata, A. Vassallo, E. Santopinto, Gluelump spectrum from Coulomb gauge QCD, Phys.Rev. D77 (2008) 056005. arXiv:0707.3156, doi:10.1103/PhysRevD.77.056005.

[194] F. E. Close, P. R. Page, Gluonic charmonium resonances at BABAR and Belle?, Phys.Lett. B628 (2005) 215-222. arXiv: hep-ph/0507199, doi:10.1016/j.physletb.2005.09.016.

[195] S.-L. Zhu, The Possible interpretations of Y(4260), Phys.Lett. B625 (2005) 212. arXiv:hep-ph/0507025, doi:10.1016/j.physletb. 2005.08 .068$.

[196] E. Braaten, How the $Z_{c}(3900)$ Reveals the Spectra of Quarkonium Hybrid and Tetraquark Mesons, Phys.Rev.Lett. 111 (2013) 162003. arXiv:1305.6905, doi:10.1103/PhysRevLett.111.162003.

[197] Yu. S. Kalashnikova, A. V. Nefediev, Spectra and decays of hybrid charmonia, Phys.Rev. D77 (2008) 054025. arXiv:0801.2036, doi : 10.1103/PhysRevD.77.054025.

[198] B. A. Li, Is X(3872) a possible candidate of hybrid meson, Phys.Lett. B605 (2005) 306-310. arXiv : hep-ph/0410264, doi : 10.1016/j . physletb.2004.11.062.

[199] N. Mahajan, Y(4140): Possible options, Phys.Lett. B679 (2009) 228-230. arXiv:0903.3107, doi:10.1016/j.physletb.2009.07. 043.

[200] P. Lacock, C. Michael, P. Boyle, P. Rowland (UKQCD collaboration), Hybrid mesons from quenched QCD, Phys.Lett. B401 (1997) 308-312. arXiv: hep-lat/9611011, doi:10.1016/S0370-2693(97)00384-5.

[201] S. Ono, Evidences for Hybrid Mesons $Q \bar{Q} G$, Z.Phys. C26 (1984) 307, [,305(1984)]. doi : $10.1007 / \mathrm{BF} 01421771$.

[202] P. R. Page, Why hybrid meson coupling to two S wave mesons is suppressed, Phys.Lett. B402 (1997) 183-188. arXiv: hep-ph/9611375, doi: 10.1016/S0370-2693(97)00438-3.

[203] E. Kou, O. Pene, Suppressed decay into open charm for the $Y(4260)$ being an hybrid, Phys.Lett. B631 (2005) 164-169. arXiv:hep-ph/ 0507119, doi:10.1016/j.physletb.2005.09.013.

[204] E. Braaten, C. Langmack, D. H. Smith, Born-Oppenheimer Approximation for the XYZ Mesons, Phys.Rev. D90 (2014) 014044. arXiv : 1402.0438, doi:10.1103/PhysRevD.90.014044.

[205] K. Marsh, R. Lewis, A lattice QCD study of generalized gluelumps, Phys.Rev. D89 (2014) 014502. arXiv:1309.1627, doi:10.1103/ PhysRevD.89.014502.

[206] R. J. Eden, P. V. Landshoff, D. I. Olive, J. C. Polkinghorne, The analytic S-matrix, Cambridge Univ. Press, Cambridge, 1966. URL https : //cds. cern. ch/record/98637

[207] D. Bugg, How Resonances can synchronise with Thresholds, J.Phys. G35 (2008) 075005. arXiv: 0802.0934, doi:10.1088/0954-3899/ $35 / 7 / 075005$. 
[208] D. Bugg, Reinterpreting several narrow 'resonances' as threshold cusps, Phys.Lett. B598 (2004) 8-14. arXiv:hep-ph/0406293, doi : $10.1016 / j$.physletb.2004.07.047.

[209] D. V. Bugg, An Explanation of Belle states $Z_{b}(10610)$ and $Z_{b}(10650)$, Europhys.Lett. 96 (2011) 11002. arXiv:1105.5492, doi : 10.1209/ 0295-5075/96/11002.

[210] E. Swanson, $Z_{b}$ and $Z_{c}$ Exotic States as Coupled Channel Cusps, Phys.Rev. D91 (3) (2015) 034009. arXiv:1409.3291, doi:10.1103/ PhysRevD.91.034009.

[211] F.-K. Guo, C. Hanhart, Q. Wang, Q. Zhao, Could the near-threshold XYZ states be simply kinematic effects?, Phys.Rev. D91 (5) (2015) 051504. arXiv: 1411.5584, doi:10.1103/PhysRevD.91.051504.

[212] A. P. Szczepaniak, Triangle Singularities and XYZ Quarkonium Peaks, Phys.Lett. B747 (2015) 410-416. arXiv:1501.01691, doi: $10.1016 / j$.physletb.2015.06.029.

[213] S. Coleman, R. E. Norton, Singularities in the physical region, Nuovo Cim. 38 (1965) 438-442. doi:10.1007/BF02750472.

[214] C. Schmid, Final-state interactions and the simulation of resonances, Phys.Rev. 154 (1967) 1363-1375. doi:10.1103/PhysRev.154. 1363. URL http://link.aps.org/doi/10.1103/PhysRev.154.1363

[215] A. P. Szczepaniak, Dalitz plot distributions in presence of triangle singularities, Phys.Lett. B757 (2016) 61-64. arXiv:1510.01789, doi: $10.1016 / j \cdot$ physletb.2016.03.064.

[216] P. Pakhlov, T. Uglov, Charged charmonium-like $Z^{+}$(4430) from rescattering in conventional $B$ decays, Phys.Lett. B748 (2015) $183-186$. arXiv:1408.5295, doi:10.1016/j.physletb.2015.06.074.

[217] I. J. R. Aitchison, Unitarity, Analyticity and Crossing Symmetry in Two- and Three-hadron Final State Interactions (2015). arXiv: 1507. 02697.

[218] F.-K. Guo, U.-G. Meißner, W. Wang, Z. Yang, How to reveal the exotic nature of the $P_{c}(4450)$, Phys.Rev. D92 (7) (2015) 071502. arXiv : 1507.04950, doi:10.1103/PhysRevD.92.071502.

[219] F.-K. Guo, U.-G. Meißner, J. Nieves, Z. Yang, Remarks on the $P_{c}$ structures and triangle singularities, Eur.Phys.J. A52 (10) (2016) 318. arXiv: 1605.05113, doi:10.1140/epja/i2016-16318-4.

[220] A. Pilloni, A. Szczepaniak, et al., to appear.

[221] M. Nielsen, F. S. Navarra, S. H. Lee, New Charmonium States in QCD Sum Rules: A Concise Review, Phys.Rept. 497 (2010) $41-83$. arXiv:0911.1958, doi:10.1016/j.physrep.2010.07.005.

[222] V. A. Novikov, L. B. Okun, M. A. Shifman, A. I. Vainshtein, M. B. Voloshin, V. I. Zakharov, Sum Rules for Charmonium and Charmed Mesons Decay Rates in Quantum Chromodynamics, Phys.Rev.Lett. 38 (1977) 626, [Erratum: Phys. Rev. Lett.38,791(1977)]. doi:10. 1103/PhysRevLett.38.626.

[223] V. A. Novikov, L. B. Okun, M. A. Shifman, A. I. Vainshtein, M. B. Voloshin, V. I. Zakharov, Charmonium and Gluons: Basic Experimental Facts and Theoretical Introduction, Phys.Rept. 41 (1978) 1-133. doi:10.1016/0370-1573(78)90120-5.

[224] M. A. Shifman, A. Vainshtein, V. I. Zakharov, QCD and Resonance Physics. Sum Rules, Nucl.Phys. B147 (1979) 385-447. doi : 10.1016/ 0550-3213(79) 90022-1.

[225] R. D. Matheus, F. Navarra, M. Nielsen, C. Zanetti, QCD Sum Rules for the $X(3872)$ as a mixed molecule-charmoniun state, Phys.Rev. D80 (2009) 056002. arXiv:0907.2683, doi:10.1103/PhysRevD.80.056002.

[226] F. Navarra, J. Dias, M. Nielsen, C. Zanetti, QCD sum rules for $Z_{c}^{+}$(3900) the state, Int.J.Mod.Phys.Conf.Ser. 26 (2014) 1460069. doi : 10.1142/S2010194514600696.

[227] W. Chen, T. G. Steele, H.-X. Chen, S.-L. Zhu, $Z_{c}(4200)^{+}$decay width as a charmonium-like tetraquark state, Eur.Phys.J. C75 (8) (2015) 358. arXiv:1501.03863, doi:10.1140/epjc/s10052-015-3578-3.

[228] S. S. Agaev, K. Azizi, H. Sundu, Strong $Z_{c}^{+}(3900) \rightarrow J / \psi \pi^{+} ; \eta_{c} \rho^{+}$decays in QCD, Phys.Rev. D93 (7) (2016) 074002. arXiv: 1601.03847 , doi: 10.1103/PhysRevD.93.074002.

[229] W. Chen, T. G. Steele, H.-X. Chen, S.-L. Zhu, Mass spectra of Zc and Zb exotic states as hadron molecules, Phys.Rev. D92 (5) (2015) 054002. arXiv:1505.05619, doi:10.1103/PhysRevD.92.054002.

[230] Z.-G. Wang, T. Huang, The $Z_{b}(10610)$ and $Z_{b}(10650)$ as axial-vector tetraquark states in the QCD sum rules, Nucl.Phys. A930 (2014) 63-85. arXiv:1312.2652, doi:10.1016/j.nuclphysa.2014.08.084.

[231] Z.-G. Wang, Tetraquark state candidates: $Y(4260), Y(4360), Y(4660)$ and $Z_{c}(4020 / 4025)$, Eur.Phys.J. C76 (7) (2016) 387. arXiv:1601. 05541, doi: 10.1140/epjc/s10052-016-4238-y.

[232] R. D. Matheus, S. Narison, M. Nielsen, J. M. Richard, Can the X(3872) be a $1^{++}$four-quark state?, Phys.Rev. D75 (2007) 014005 . arXiv : hep-ph/0608297, doi:10.1103/PhysRevD.75.014005.

[233] R. Gupta, Introduction to lattice QCD, in: Probing the standard model of particle interactions. Proceedings, Summer School in Theoretical Physics, NATO Advanced Study Institute, 68th session, Les Houches, France, July 28-September 5, 1997. Pt. 1, 2, 1997, pp. 83-219. arXiv:hep-lat/9807028.

URL http: //alice.cern. ch/format/showfull?sysnb=0284452

[234] C. Gattringer, C. Lang, Quantum Chromodynamics on the Lattice: An Introductory Presentation, Lecture Notes in Physics, Springer Berlin Heidelberg, 2009. URL https://books.google.com/books?id=12hZKnlYDxoC

[235] M. Lüscher, U. Wolff, How to Calculate the Elastic Scattering Matrix in Two-dimensional Quantum Field Theories by Numerical Simulation, Nucl.Phys. B339 (1990) 222-252. doi : 10.1016/0550-3213(90)90540-T.

[236] M. Lüscher, Two particle states on a torus and their relation to the scattering matrix, Nucl.Phys. B354 (1991) 531-578. doi:10.1016/ 0550-3213 (91) 90366-6.

[237] M. Lüscher, Signatures of unstable particles in finite volume, Nucl.Phys. B364 (1991) 237-254. doi : 10.1016/0550-3213 (91) 90584-K.

[238] L. Giusti, A. L. Guerrieri, S. Petrarca, A. Rubeo, M. Testa, Color structure of Yang-Mills theory with static sources in a periodic box, Phys.Rev. D92 (3) (2015) 034515. arXiv: 1508.00152, doi : 10.1103/PhysRevD.92.034515. 
[239] P. Bicudo, M. Wagner (European Twisted Mass collaboration), Lattice QCD signal for a bottom-bottom tetraquark, Phys.Rev. D87 (11) (2013) 114511. arXiv:1209.6274, doi:10.1103/PhysRevD.87.114511.

[240] Z. S. Brown, K. Orginos, Tetraquark bound states in the heavy-light heavy-light system, Phys.Rev. D86 (2012) 114506. arXiv: 1210.1953 , doi:10.1103/PhysRevD.86.114506.

[241] P. Bicudo, K. Cichy, A. Peters, B. Wagenbach, M. Wagner, Evidence for the existence of $u d \bar{b} \bar{b}$ and the non-existence of $s s \bar{b} \bar{b}$ and $c c \bar{b} \bar{b}$ tetraquarks from lattice QCD, Phys.Rev. D92 (1) (2015) 014507. arXiv: 1505.00613, doi:10.1103/PhysRevD .92.014507.

[242] A. Francis, R. J. Hudspith, R. Lewis, K. Maltman, Doubly bottom strong-interaction stable tetraquarks from lattice QCD (2016). arXiv: 1607.05214

[243] S. Prelovsek, L. Leskovec, Evidence for $X$ (3872) from $D D^{*}$ scattering on the lattice, Phys.Rev.Lett. 111 (2013) 192001. arXiv: 1307.5172, doi:10.1103/PhysRevLett.111.192001.

[244] A. Esposito, M. Papinutto, A. Pilloni, A. Polosa, N. Tantalo, Doubly charmed tetraquarks in $B_{c}$ and $\Xi_{b c}$ decays, Phys.Rev. D88 (5) (2013) 054029. arXiv: 1307.2873, doi:10.1103/PhysRevD.88.054029.

[245] A. Guerrieri, M. Papinutto, A. Pilloni, A. Polosa, N. Tantalo, Flavored tetraquark spectroscopy, PoS LATTICE2014 (2014) 106. arXiv: 1411.2247.

[246] Y. Ikeda, B. Charron, S. Aoki, T. Doi, T. Hatsuda, T. Inoue, N. Ishii, K. Murano, H. Nemura, K. Sasaki, Charmed tetraquarks $T_{c c}$ and $T_{c s}$ from dynamical lattice QCD simulations, Phys.Lett. B729 (2014) 85-90. arXiv:1311.6214, doi:10.1016/j.physletb.2014.01.002.

[247] K. J. Juge, J. Kuti, C. J. Morningstar, Gluon excitations of the static quark potential and the hybrid quarkonium spectrum, Nucl.Phys.Proc.Suppl. 63 (1998) 326-331. arXiv : hep-lat/9709131, doi:10.1016/S0920-5632 (97) 00759-7.

[248] K. J. Juge, J. Kuti, C. J. Morningstar, Ab initio study of hybrid anti-b g b mesons, Phys.Rev.Lett. 82 (1999) 4400-4403. arXiv:hep-ph/ 9902336, doi:10.1103/PhysRevLett.82.4400.

[249] L. Liu, et al. (Hadron Spectrum collaboration), Excited and exotic charmonium spectroscopy from lattice QCD, JHEP 1207 (2012) 126. arXiv:1204.5425, doi:10.1007/JHEP07(2012) 126 .

[250] S. F. Radford, W. W. Repko, Potential model calculations and predictions for heavy quarkonium, Phys.Rev. D75 (2007) 074031. arXiv: hep-ph/0701117, doi:10.1103/PhysRevD.75.074031.

[251] M. Ablikim, et al. (BES III collaboration), Confirmation of a charged charmoniumlike state $Z_{c}(3885)^{\mp}$ in $e^{+} e^{-} \rightarrow \pi^{ \pm}\left(D \bar{D}^{*}\right)^{\mp}$ with double $D$ tag, Phys.Rev. D92 (9) (2015) 092006. arXiv: 1509.01398, doi:10.1103/PhysRevD.92.092006.

[252] T. Xiao, S. Dobbs, A. Tomaradze, K. K. Seth, Observation of the Charged Hadron $Z_{c}^{ \pm}(3900)$ and Evidence for the Neutral $Z_{c}^{0}(3900)$ in $e^{+} e^{-} \rightarrow \pi \pi J \psi$ at $\sqrt{s}=4170 \mathrm{MeV}$, Phys.Lett. B727 (2013) 366-370. arXiv:1304.3036, doi:10.1016/j.physletb.2013.10.041.

[253] M. Ablikim, et al. (BES III collaboration), Observation of a Neutral Structure near the $D \bar{D}^{*}$ Mass Threshold in $e^{+} e^{-} \rightarrow\left(D \bar{D}^{*}\right)^{0} \pi^{0}$ at $\sqrt{s}=$ 4.226 and $4.257 \mathrm{GeV}$, Phys.Rev.Lett. 115 (22) (2015) 222002. arXiv: 1509.05620, doi:10.1103/PhysRevLett.115. 222002.

[254] M. Ablikim, et al. (BESIII collaboration), Observation of a charged charmoniumlike structure in $e^{+} e^{-} \rightarrow\left(D^{*} \bar{D}^{*}\right)^{ \pm} \pi^{\mp}$ at $\sqrt{s}=4.26 \mathrm{GeV}$, Phys.Rev.Lett. 112 (2014) 132001. arXiv:1308.2760, doi:10.1103/PhysRevLett.112.132001.

[255] M. Ablikim, et al. (BESIII collaboration), Observation of $e^{+} e^{-} \rightarrow \pi^{0} \pi^{0} h_{c}$ and a neutral charmoniumlike structure $Z_{c}(4020)^{0}$, Phys.Rev.Lett. 113 (21) (2014) 212002. arXiv: 1409.6577, doi:10.1103/PhysRevLett.113.212002.

[256] M. Ablikim, et al. (BES III collaboration), Observation of a neutral charmoniumlike state $Z_{c}(4025)^{0}$ in $e^{+} e^{-} \rightarrow\left(D^{*} \bar{D}^{*}\right)^{0} \pi^{0}$, Phys.Rev.Lett. 115 (18) (2015) 182002. arXiv: 1507.02404, doi:10.1103/PhysRevLett.115.182002.

[257] K. Abe, et al. (Belle collaboration), Observation of a near-threshold $\omega J \psi$ mass enhancement in exclusive $B \rightarrow K \omega J \psi$ decays, Phys.Rev.Lett. 94 (2005) 182002. arXiv:hep-ex/0408126, doi:10.1103/PhysRevLett.94.182002.

[258] B. Aubert, et al. (BABAR collaboration), Observation of $Y(3940) \rightarrow J \psi \omega$ in $B \rightarrow J \psi \omega K$ at BABAR, Phys.Rev.Lett. 101 (2008) 082001. arXiv:0711.2047, doi:10.1103/PhysRevLett.101.082001.

[259] P. del Amo Sanchez, et al. (BABAR collaboration), Evidence for the decay $X(3872) \rightarrow J \psi \omega$, Phys.Rev. D82 (2010) 011101. arXiv: 1005.5190, doi: 10.1103/PhysRevD.82.011101.

[260] S. Uehara, et al. (Belle collaboration), Observation of a charmonium-like enhancement in the $\gamma \gamma \rightarrow \omega J \psi$ process, Phys.Rev.Lett. 104 (2010) 092001. arXiv:0912.4451, doi:10.1103/PhysRevLett.104.092001.

[261] J. P. Lees, et al. (BABAR collaboration), Study of $X(3915) \rightarrow J \psi \omega$ in two-photon collisions, Phys.Rev. D86 (2012) 072002. arXiv: 1207.2651, doi:10.1103/PhysRevD.86.072002.

[262] K. Abe, et al. (Belle collaboration), Observation of a new charmonium state in double charmonium production in $e^{+} e^{-}$annihilation at $\sqrt{s} \approx 10.6 \mathrm{GeV}$, Phys.Rev.Lett. 98 (2007) 082001. arXiv: hep-ex/0507019, doi:10.1103/PhysRevLett.98.082001.

[263] P. Pakhlov, et al. (Belle collaboration), Production of new charmoniumlike states in $e^{+} e^{-} \rightarrow J \psi D^{*} \bar{D}^{*}$ at $\sqrt{s} \approx 10.6 \mathrm{GeV}$, Phys.Rev.Lett. 100 (2008) 202001. arXiv:0708.3812, doi:10.1103/PhysRevLett.100.202001.

[264] C. Z. Yuan, et al. (Belle collaboration), Measurement of $e^{+} e^{-} \rightarrow \pi^{+} \pi^{-} J \psi$ Cross Section via Initial State Radiation at Belle, Phys.Rev.Lett. 99 (2007) 182004. arXiv:0707.2541, doi:10.1103/PhysRevLett.99.182004.

[265] R. Mizuk, et al. (Belle collaboration), Observation of two resonance-like structures in the $\pi^{+} \chi_{c 1}$ mass distribution in exclusive $\bar{B}^{0} \rightarrow K^{-} \pi^{+} \chi_{c 1}$ decays, Phys.Rev. D78 (2008) 072004. arXiv:0806.4098, doi:10.1103/PhysRevD.78.072004.

[266] J. P. Lees, et al. (BABAR collaboration), Search for the $Z_{1}(4050)^{+}$and $Z_{2}(4250)^{+}$states in $\bar{B}^{0} \rightarrow \chi_{c 1} K^{-} \pi^{+}$and $B^{+} \rightarrow \chi_{c 1} K_{S}^{0} \pi^{+}$, Phys.Rev. D85 (2012) 052003. arXiv:1111.5919, doi:10.1103/PhysRevD.85.052003.

[267] X. Wang, C. Yuan, C. Shen, P. Wang, A. Abdesselam, et al. (Belle collaboration), Measurement of $e^{+} e^{-} \rightarrow \pi^{+} \pi^{-} \psi(2 S)$ via Initial State Radiation at Belle, Phys.Rev. D91 (11) (2015) 112007. arXiv: 1410.7641, doi:10.1103/PhysRevD.91.112007.

[268] K. Chilikin, et al. (Belle collaboration), Observation of a new charged charmonium-like state in $B \rightarrow J \psi K \pi$ decays, Phys.Rev. D90 (11) (2014) 112009. arXiv: 1408.6457, doi:10.1103/PhysRevD.90.112009.

[269] M. Ablikim, et al. (BESIII collaboration), Study of $e^{+} e^{-} \rightarrow \omega \chi_{c J}$ at center-of-mass energies from 4.21 to 4.42 GeV, Phys.Rev.Lett. 114 (9) (2015) 092003. arXiv: 1410.6538, doi:10.1103/PhysRevLett.114.092003.

[270] B. Aubert, et al. (BABAR collaboration), Observation of a broad structure in the $\pi^{+} \pi^{-} J \psi$ mass spectrum around 4.26- GeV $/ c^{2}$, Phys.Rev.Lett. 95 (2005) 142001. arXiv: hep-ex/0506081, doi:10.1103/PhysRevLett.95.142001. 
[271] J. P. Lees, et al. (BABAR collaboration), Study of the reaction $e^{+} e^{-} \rightarrow J \psi \pi^{+} \pi^{-}$via initial-state radiation at BABAR, Phys.Rev. D86 (2012) 051102. arXiv: 1204.2158, doi:10.1103/PhysRevD.86.051102.

[272] T. E. Coan, et al. (CLEO collaboration), Charmonium decays of $Y(4260), \psi(4160)$ and $\psi(4040)$, Phys.Rev.Lett. 96 (2006) 162003. arXiv: hep-ex/0602034, doi:10.1103/PhysRevLett.96.162003.

[273] Q. He, et al. (CLEO collaboration), Confirmation of the Y(4260) resonance production in ISR, Phys.Rev. D74 (2006) 091104. arXiv: hep-ex/0611021, doi:10.1103/PhysRevD.74.091104.

[274] C. P. Shen, et al. (Belle collaboration), Evidence for a new resonance and search for the $Y(4140)$ in $\gamma \gamma \rightarrow \phi J \psi$, Phys.Rev.Lett. 104 (2010) 112004. arXiv:0912.2383, doi:10.1103/PhysRevLett.104.112004.

[275] X. L. Wang, et al. (Belle collaboration), Observation of Two Resonant Structures in $e^{+} e^{-} \rightarrow \pi^{+} \pi^{-} \psi(2 S)$ via Initial State Radiation at Belle, Phys.Rev.Lett. 99 (2007) 142002. arXiv:0707.3699, doi:10.1103/PhysRevLett.99.142002.

[276] J. Lees, et al. (BABAR collaboration), Study of the reaction $e^{+} e^{-} \rightarrow \psi(2 S) \pi^{+} \pi^{-}$via initial state radiation at BABAR, Phys.Rev. D89 (2014) 111103. arXiv:1211.6271, doi:10.1103/PhysRevD.89.111103.

[277] R. Mizuk, et al. (Belle collaboration), Dalitz analysis of $B \rightarrow K \pi \psi^{\prime}$ decays and the Z(4430) ${ }^{+}$, Phys.Rev. D80 (2009) 031104. arXiv: 0905.2869, doi:10.1103/PhysRevD.80.031104.

[278] K. Chilikin, et al. (Belle collaboration), Experimental constraints on the spin and parity of the Z(4430) ${ }^{+}$, Phys.Rev. D88 (2013) 074026 arXiv: 1306.4894, doi:10.1103/PhysRevD.88.074026.

[279] B. Aubert, et al. (BABAR collaboration), Search for the $Z(4430)^{-}$at BABAR, Phys.Rev. D79 (2009) 112001. arXiv:0811.0564, doi: 10.1103/PhysRevD.79.112001.

[280] R. Aaij, et al. (LHCb collaboration), Observation of the resonant character of the Z(4430)- state, Phys.Rev.Lett. 112 (2014) 222002. arXiv : 1404.1903, doi:10.1103/PhysRevLett.112.222002.

[281] G. Pakhlova, et al. (Belle collaboration), Observation of a near-threshold enhancement in the $e^{+} e^{-} \rightarrow \Lambda_{c}^{+} \Lambda_{c}^{-}$cross section using initial-state radiation, Phys.Rev.Lett. 101 (2008) 172001. arXiv:0807.4458, doi:10.1103/PhysRevLett.101.172001.

[282] R. Aaij, et al. (LHCb collaboration), Search for structure in the $B_{s}^{0} \pi^{ \pm}$invariant mass spectrum, Phys.Rev.Lett. 117 (15) (2016) 152003 . arXiv:1608.00435, doi:10.1103/PhysRevLett.117.152003.

[283] A. Bondar, et al. (Belle collaboration), Observation of two charged bottomonium-like resonances in $Y(5 S)$ decays, Phys.Rev.Lett. 108 (2012) 122001. arXiv:1110.2251, doi:10.1103/PhysRevLett.108.122001.

[284] I. Adachi, et al. (Belle collaboration), Study of Three-Body $Y(10860)$ Decays (2012). arXiv: 1209.6450.

[285] A. Garmash, et al. (Belle collaboration), Observation of $Z_{b}(10610)$ and $Z_{b}^{\prime}(10650)$ Decaying to $B$ Mesons, Phys.Rev.Lett. 116 (21) (2016) 212001. arXiv:1512.07419, doi:10.1103/PhysRevLett.116.212001.

[286] P. Krokovny, et al. (Belle collaboration), First observation of the $Z_{b}^{0}(10610)$ in a Dalitz analysis of $\Upsilon(10860) \rightarrow \Upsilon(n S) \pi^{0} \pi^{0}$, Phys.Rev. D88 (5) (2013) 052016. arXiv: 1308.2646, doi: 10.1103/PhysRevD.88.052016.

[287] C.-N. Yang, Selection Rules for the Dematerialization of a Particle Into Two Photons, Phys.Rev. 77 (1950) 242-245. doi:10.1103/ PhysRev.77.242.

[288] A. Bevan, et al. (Belle, BaBar collaboration), The Physics of the B Factories, Eur.Phys.J. C74 (2014) 3026. arXiv:1406.6311, doi : 10.1140/epjc/s10052-014-3026-9.

[289] R. Faccini, A. Pilloni, A. D. Polosa, Exotic Heavy Quarkonium Spectroscopy: A Mini-review, Mod.Phys.Lett. A27 (2012) 1230025. arXiv : 1209.0107, doi:10.1142/S021773231230025X.

[290] R. A. Briceño, et al., Issues and Opportunities in Exotic Hadrons, Chin.Phys. C40 (4) (2016) 042001. arXiv: 1511.06779, doi : 10.1088/ 1674-1137/40/4/042001.

[291] M. F. M. Lutz, et al., Resonances in QCD, Nucl.Phys. A948 (2016) 93-105. arXiv:1511.09353, doi:10.1016/j.nuclphysa.2016. 01.070 .

[292] K. Abe, et al. (Belle collaboration), Observation of large CP violation in the neutral $B$ meson system, Phys.Rev.Lett. 87 (2001) 091802. arXiv:hep-ex/0107061, doi:10.1103/PhysRevLett.87.091802.

[293] B. Aubert, et al. (BABAR collaboration), Study of the $B \rightarrow J \psi K^{-} \pi^{+} \pi^{-}$decay and measurement of the $B \rightarrow X(3872) K^{-}$branching fraction, Phys.Rev. D71 (2005) 071103. arXiv: hep-ex/0406022, doi:10.1103/PhysRevD.71.071103.

[294] V. Abazov, et al. (D $\varnothing$ collaboration), Observation and properties of the $X(3872)$ decaying to $J \psi \pi^{+} \pi^{-}$in $p \bar{p}$ collisions at $\sqrt{s}=1.96 \mathrm{TeV}$ Phys.Rev.Lett. 93 (2004) 162002. arXiv: hep-ex/0405004, doi:10.1103/PhysRevLett.93.162002.

[295] S.-K. Choi, S. L. Olsen, K. Trabelsi, I. Adachi, H. Aihara, et al., Bounds on the width, mass difference and other properties of $X(3872) \rightarrow$ $\pi^{+} \pi^{-} J \psi$ decays, Phys.Rev. D84 (2011) 052004. arXiv:1107.0163, doi:10.1103/PhysRevD.84.052004.

[296] A. Abulencia, et al. (CDF collaboration), Measurement of the dipion mass spectrum in $X(3872) \rightarrow J \psi \pi^{+} \pi^{-}$decays., Phys.Rev.Lett. 96 (2006) 102002. arXiv:hep-ex/0512074, doi:10.1103/PhysRevLett.96.102002.

[297] A. Abulencia, et al. (CDF collaboration), Analysis of the quantum numbers $J^{P C}$ of the $X(3872)$, Phys.Rev.Lett. 98 (2007) 132002. arXiv: hep-ex/0612053, doi:10.1103/PhysRevLett.98.132002.

[298] K. Abe, et al. (Belle collaboration), Evidence for $X(3872) \rightarrow \gamma J \psi$ and the sub-threshold decay $X(3872) \rightarrow \omega J \psi(2005)$. arXiv $:$ hep-ex/ 0505037.

[299] B. Aubert, et al. (BABAR collaboration), Search for $B^{+} \rightarrow X(3872) K^{+}, X(3872) \rightarrow J \psi \gamma$, Phys.Rev. D74 (2006) 071101. arXiv: hep-ex/ 0607050, doi:10.1103/PhysRevD.74.071101.

[300] B. Aubert, et al. (BABAR collaboration), Evidence for $X(3872) \rightarrow \psi(2 S) \gamma$ in $B^{ \pm} \rightarrow X(3872) K^{ \pm}$decays, and a study of $B \rightarrow c \bar{c} \gamma K$, Phys.Rev.Lett. 102 (2009) 132001. arXiv:0809.0042, doi:10.1103/PhysRevLett.102.132001.

[301] V. Bhardwaj, et al. (Belle collaboration), Observation of $X(3872) \rightarrow J \psi \gamma$ and search for $X(3872) \rightarrow \psi^{\prime} \gamma$ in $B$ decays, Phys.Rev.Lett. 107 (2011) 9. arXiv: 1105.0177, doi:10.1103/PhysRevLett.107.091803.

[302] S. L. Olsen (Belle collaboration), Search for a charmonium assignment for the X(3872), Int.J.Mod.Phys. A20 (2005) 240-249. arXiv: hep-ex/0407033, doi:10.1142/S0217751X05021403.

[303] K. Abe, et al. (Belle collaboration), Experimental constraints on the possible $J^{P C}$ quantum numbers of the $X(3872)(2005)$. arXiv : hep-ex/ 
0505038

[304] T. J. Burns, F. Piccinini, A. D. Polosa, C. Sabelli, The $2^{-+}$assignment for the X(3872), Phys.Rev. D82 (2010) 074003. arXiv : 1008.0018, doi:10.1103/PhysRevD.82.074003.

[305] C. Hanhart, Y. S. Kalashnikova, A. E. Kudryavtsev, A. V. Nefediev, Remarks on the quantum numbers of $X(3872)$ from the invariant mass distributions of the $\rho J \psi$ and $\omega J \psi$ final states, Phys.Rev. D85 (2012) 011501. arXiv:1111.6241, doi:10.1103/PhysRevD.85.011501.

[306] R. Faccini, F. Piccinini, A. Pilloni, A. Polosa, The Spin of the X(3872), Phys.Rev. D86 (2012) 054012. arXiv: 1204.1223, doi:10.1103/ PhysRevD.86.054012.

[307] E. Braaten, D. Kang, J/ $\psi \omega$ Decay Channel of the X(3872) Charm Meson Molecule, Phys.Rev. D88 (1) (2013) 014028. arXiv: 1305.5564, doi: 10.1103/PhysRevD.88.014028.

[308] R. Aaij, et al. (LHCb collaboration), Determination of the $X(3872)$ meson quantum numbers, Phys.Rev.Lett. 110 (2013) 222001. arXiv : 1302.6269, doi:10.1103/PhysRevLett.110.222001.

[309] R. Aaij, et al. (LHCb collaboration), Quantum numbers of the $X(3872)$ state and orbital angular momentum in its $\rho^{0} J \psi$ decay, Phys.Rev. D92 (1) (2015) 011102. arXiv: 1504.06339, doi:10.1103/PhysRevD.92.011102.

[310] G. Gokhroo, et al. (Belle collaboration), Observation of a near-threshold $D^{0} \bar{D}^{0} \pi^{0}$ enhancement in $B \rightarrow D^{0} \bar{D}^{0} \pi^{0} K$ decay, Phys.Rev.Lett. 97 (2006) 162002. arXiv: hep-ex/0606055, doi:10.1103/PhysRevLett.97.162002.

[311] B. Aubert, et al. (BABAR collaboration), Study of Resonances in Exclusive $B$ Decays to $\bar{D}^{(*)} D^{(*)} K$, Phys.Rev. D77 (2008) 011102 . arXiv : 0708.1565, doi:10.1103/PhysRevD.77.011102.

[312] T. Aushev, et al. (Belle collaboration), Study of the $B \rightarrow X(3872)\left(D^{* 0} \bar{D}^{0}\right) K$ decay, Phys.Rev. D81 (2010) 031103. arXiv:0810.0358, doi:10.1103/PhysRevD.81.031103.

[313] P. Artoisenet, E. Braaten, D. Kang, Using Line Shapes to Discriminate between Binding Mechanisms for the X(3872), Phys.Rev. D82 (2010) 014013. arXiv:1005.2167, doi:10.1103/PhysRevD.82.014013.

[314] C. Hanhart, Y. S. Kalashnikova, A. V. Nefediev, Lineshapes for composite particles with unstable constituents, Phys.Rev. D81 (2010) 094028. arXiv:1002.4097, doi:10.1103/PhysRevD.81.094028.

[315] C. Hanhart, Y. S. Kalashnikova, A. V. Nefediev, Interplay of quark and meson degrees of freedom in a near-threshold resonance: multichannel case, Eur.Phys.J. A47 (2011) 101-110. arXiv:1106.1185, doi:10.1140/epja/i2011-11101-9.

[316] R. Aaij, et al. (LHCb collaboration), Evidence for the decay $X(3872) \rightarrow \psi(2 S) \gamma$, Nucl.Phys. B886 (2014) 665-680. arXiv:1404.0275, doi:10.1016/j.nuclphysb.2014.06.011.

[317] E. S. Swanson, Diagnostic decays of the X(3872), Phys.Lett. B598 (2004) 197-202. arXiv:hep-ph/0406080, doi:10.1016/j. physletb.2004.07.059.

[318] E. Braaten, An Estimate of the Partial Width for X(3872) into p p , Phys.Rev. D77 (2008) 034019. arXiv:0711.1854, doi:10.1103/ PhysRevD.77.034019.

[319] R. Aaij, et al. (LHCb collaboration), Measurements of the branching fractions of $B^{+} \rightarrow p \bar{p} K^{+}$decays, Eur.Phys.J. C73 (2013) 2462. arXiv: 1303.7133, doi:10.1140/epjc/s10052-013-2462-2.

[320] R. Aaij, et al. (LHCb collaboration), Observation of $\eta_{c}(2 S) \rightarrow p \bar{p}$ and search for $X(3872) \rightarrow p \bar{p}$ decays (2016). arXiv: 1607.06446 .

[321] I. Adachi, et al. (Belle collaboration), Study of X(3872) in $B$ meson decays (2008). arXiv:0809.1224.

[322] A. Bala, et al. (Belle collaboration), Observation of $X(3872)$ in $B \rightarrow X(3872) K \pi$ decays, Phys.Rev. D91 (5) (2015) 051101. arXiv: 1501.06867, doi:10.1103/PhysRevD.91.051101.

[323] A. Guskov, Observation of exclusive production of a charmonium and a charmonium-like state in the reaction $\mu+n \rightarrow \mu+\left(J / \psi \pi^{+} \pi^{-}\right) \pi^{ \pm} n^{\prime}$, talk at QWG2016, https://indico.hep.pnnl.gov/event/0/session/7/contribution/17/material/slides/0.pdf.

[324] B. Aubert, et al. (BABAR collaboration), Measurements of the absolute branching fractions of $B^{ \pm} \rightarrow K^{ \pm} X_{c \bar{c}}$, Phys.Rev.Lett. 96 (2006) 052002. arXiv:hep-ex/0510070, doi:10.1103/PhysRevLett.96.052002.

[325] Global fit for $X(3872)$ URL http://www.roma1.infn.it/ pillonia/PhysRept/

[326] M. Aaboud, et al. (ATLAS collaboration), Measurements of $\psi(2 S)$ and $X(3872) \rightarrow J / \psi \pi^{+} \pi^{-}$production in $p p$ collisions at $\sqrt{s}=8$ TeV with the ATLAS detector (2016). arXiv: 1610.09303.

[327] B. Aubert, et al. (BABAR collaboration), Study of $J \psi \pi^{+} \pi^{-}$states produced in $B^{0} \rightarrow J \psi \pi^{+} \pi^{-} K^{0}$ and $B^{-} \rightarrow J \psi \pi^{+} \pi^{-} K^{-}$, Phys.Rev. D73 (2006) 011101. arXiv: hep-ex/0507090, doi:10.1103/PhysRevD.73.011101.

[328] B. Aubert, et al. (BABAR collaboration), A Study of $B \rightarrow X(3872) K$, with $X(3872) \rightarrow J \psi \pi^{+} \pi^{-}$, Phys.Rev. D77 (2008) 111101 . arXiv : 0803.2838, doi:10.1103/PhysRevD.77.111101.

[329] T. Aaltonen, et al. (CDF collaboration), Precision Measurement of the $X(3872)$ Mass in $J \psi \pi^{+} \pi^{-}$Decays, Phys.Rev.Lett. 103 (2009) 152001 . arXiv:0906.5218, doi:10.1103/PhysRevLett.103.152001.

[330] B. Aubert, et al. (BABAR collaboration), Search for a charged partner of the $X(3872)$ in the $B$ meson decay $B \rightarrow X^{-} K, X^{-} \rightarrow J \psi \pi^{-} \pi^{0}$, Phys.Rev. D71 (2005) 031501. arXiv: hep-ex/0412051, doi:10.1103/PhysRevD.71.031501.

[331] M. Voloshin, Two charmoniumlike charged axial resonances near $3885 \mathrm{MeV}$, Phys.Rev. D94 (7) (2016) 074010. arXiv:1607.04887, doi: 10.1103/PhysRevD.94.074010.

[332] The "Lifetime" Distribution of $X(3872)$ Mesons Produced in $\bar{p} p$ Collisions at CDF, cDF note 7159. URL http://www-cdf.fnal.gov/physics/new/bottom/051020.blessed-X3872/XLife/xlonglivedWWW.ps

[333] V. Bhardwaj, et al. (Belle collaboration), Evidence of a new narrow resonance decaying to $\chi_{c 1} \gamma$ in $B \rightarrow \chi_{c 1} \gamma K$, Phys.Rev.Lett. 111 (2013) 032001. arXiv: 1304.3975, doi:10.1103/PhysRevLett.111.032001.

[334] K. Abe, et al. (Belle collaboration), Search for resonant $B^{ \pm} \rightarrow K^{ \pm} h \rightarrow K^{ \pm} \gamma \gamma$ Decays at Belle, Phys.Lett. B662 (2008) 323-329. arXiv : hep-ex/0608037, doi:10.1016/j.physletb.2008.03.029.

[335] B. Aubert, et al. (BABAR collaboration), Observation of the decay $B \rightarrow J \psi \eta K$ and search for $X(3872) \rightarrow J \psi \eta$, Phys.Rev.Lett. 93 (2004) 041801. arXiv: hep-ex/0402025, doi:10.1103/PhysRevLett.93.041801.

[336] K. Abe, et al. (Belle collaboration), Observation of $B^{+} \rightarrow \psi(3770) K^{+}$, Phys.Rev.Lett. 93 (2004) 051803. arXiv:hep-ex/0307061, 
doi: 10.1103/PhysRevLett.93.051803.

[337] S. Dobbs, et al. (CLEO collaboration), Search for X(3872) in $\gamma \gamma$ fusion and ISR at CLEO, Phys.Rev.Lett. 94 (2005) 032004. arXiv : hep-ex/0410038, doi:10.1103/PhysRevLett.94.032004.

[338] M. Ablikim, et al. (BES III collaboration), An improved limit for $\Gamma_{e e}$ of $X(3872)$ and $\Gamma_{e e}$ measurement of $\psi(3686)$, Phys.Lett. B749 (2015) 414-420. arXiv:1505.02559, doi:10.1016/j.physletb.2015.08.013.

[339] M. Ablikim, et al. (BESIII collaboration), Observation of $Z_{c}(3900)^{0}$ in $e^{+} e^{-} \rightarrow \pi^{0} \pi^{0} J / \psi$, Phys.Rev.Lett. 115 (11) (2015) 112003 . arXiv: 1506.06018, doi:10.1103/PhysRevLett.115.112003.

[340] M. Ablikim, et al. (BES III collaboration), Search for $Z_{c}(3900)^{ \pm} \rightarrow \omega \pi^{ \pm}$, Phys.Rev. D92 (3) (2015) 032009. arXiv:1507.02068, doi: 10.1103/PhysRevD.92.032009.

[341] C. Adolph, et al. (COMPASS collaboration), Search for exclusive photoproduction of Z $Z_{c}^{ \pm}$(3900) at COMPASS, Phys.Lett. B742 (2015) 330-334. arXiv: 1407.6186, doi:10.1016/j.physletb.2015.01.042.

[342] K.-F. Chen, et al. (Belle collaboration), Observation of anomalous $\Upsilon(1 S) \pi^{+} \pi^{-}$and $\Upsilon(2 S) \pi^{+} \pi^{-}$production near the $\Upsilon(5 S)$ resonance, Phys.Rev.Lett. 100 (2008) 112001. arXiv:0710.2577, doi:10.1103/PhysRevLett.100.112001.

[343] I. Adachi, et al. (Belle collaboration), First observation of the $P$-wave spin-singlet bottomonium states $h_{b}(1 P)$ and $h_{b}(2 P)$, Phys.Rev.Lett. 108 (2012) 032001. arXiv:1103.3419, doi:10.1103/PhysRevLett.108.032001.

[344] A. Ali, L. Maiani, A. Polosa, V. Riquer, Hidden-Beauty Charged Tetraquarks and Heavy Quark Spin Conservation, Phys.Rev. D91 (1) (2015) 017502. arXiv:1412.2049, doi:10.1103/PhysRevD.91.017502.

[345] A. Garmash, et al. (Belle collaboration), Amplitude analysis of $e^{+} e^{-} \rightarrow \Upsilon(n S) \pi^{+} \pi^{-}$at $\sqrt{s}=10.865 \mathrm{GeV}$, Phys.Rev. D91 (7) (2015) 072003. arXiv:1403.0992, doi:10.1103/PhysRevD.91.072003.

[346] A. Ali, C. Hambrock, W. Wang, Tetraquark Interpretation of the Charged Bottomonium-like states $Z_{b}^{ \pm}(10610)$ and $Z_{b}^{ \pm}(10650)$ and Implications, Phys.Rev. D85 (2012) 054011. arXiv:1110.1333, doi:10.1103/PhysRevD.85.054011.

[347] A. Ali, C. Hambrock, I. Ahmed, M. J. Aslam, A case for hidden $b \bar{b}$ tetraquarks based on $e^{+} e^{-} \rightarrow b \bar{b}$ cross section between $\sqrt{s}=10.54$ and 11.20 GeV, Phys.Lett. B684 (2010) 28-39. arXiv:0911.2787, doi:10.1016/j ·physletb.2009.12.053.

[348] A. Ali, C. Hambrock, M. J. Aslam, A Tetraquark interpretation of the BELLE data on the anomalous $\Upsilon(1 S) \pi^{+} \pi^{-}$and $\Upsilon(2 S) \pi^{+} \pi^{-}$production near the $\Upsilon(5 S)$ resonance, Phys.Rev.Lett. 104 (2010) 162001, [Erratum: Phys.Rev. Lett.107,049903(2011)]. arXiv:0912.5016, doi: 10.1103/PhysRevLett.104.162001, 10.1103/PhysRevLett.107.049903.

[349] B. Aubert, et al. (BaBar collaboration), Measurement of the $e^{+} e^{-} \rightarrow b \bar{b}$ cross section between $\sqrt{s}=10.54-\mathrm{GeV}$ and $11.20-\mathrm{GeV}$, Phys.Rev.Lett. 102 (2009) 012001. arXiv:0809.4120, doi:10.1103/PhysRevLett.102.012001.

[350] D. Santel, et al. (Belle collaboration), Measurements of the $\Upsilon(10860)$ and $\Upsilon(11020)$ resonances via $\sigma\left(e^{+} e^{-} \rightarrow \Upsilon(n S) \pi^{+} \pi^{-}\right)$, Phys.Rev. D93 (1) (2016) 011101. arXiv: 1501.01137, doi: 10.1103/PhysRevD.93.011101.

[351] A. Ali, Multiquark Hadrons - A New Facet of QCD, in: 14th Regional Conference on Mathematical Physics Islamabad, Pakistan, November 9-14, 2015, 2016. arXiv: 1605.05954.

URL http://inspirehep.net/record/1462270/files/arXiv: 1605.05954.pdf

[352] N. A. Tornqvist, The $\Upsilon$ (5S) Mass and $e^{+} e^{-} \rightarrow B \bar{B}, B \bar{B}^{*}, B^{*} \bar{B}^{*}$ as Sensitive Tests of the Unitarized Quark Model, Phys.Rev.Lett. 53 (1984) 878. doi:10.1103/PhysRevLett.53.878.

[353] F.-K. Guo, C. Hidalgo-Duque, J. Nieves, M. P. Valderrama, Consequences of Heavy Quark Symmetries for Hadronic Molecules, Phys.Rev. D88 (2013) 054007. arXiv: 1303.6608, doi:10.1103/PhysRevD.88.054007.

[354] X. H. He, et al. (Belle collaboration), Observation of $e^{+} e^{-} \rightarrow \pi^{+} \pi^{-} \pi^{0} \chi_{b J}$ and Search for $X_{b} \rightarrow \omega \Upsilon(1 S)$ at $\sqrt{s}=10.867 \mathrm{GeV}$, Phys.Rev.Lett. 113 (14) (2014) 142001. arXiv: 1408.0504, doi:10.1103/PhysRevLett.113.142001.

[355] G. Aad, et al. (ATLAS collaboration), Search for the $X_{b}$ and other hidden-beauty states in the $\pi^{+} \pi^{-} \Upsilon(1 S)$ channel at ATLAS, Phys.Lett. B740 (2015) 199-217. arXiv: 1410.4409, doi:10.1016/j.physletb.2014.11.055.

[356] J. E. Augustin, et al. (SLAC-SP-017 collaboration), Discovery of a Narrow Resonance in $e^{+} e^{-}$Annihilation, Phys.Rev.Lett. 33 (1974) 1406-1408, [Adv. Exp. Phys.5,141(1976)]. doi:10.1103/PhysRevLett.33.1406.

[357] M. Cleven, Q. Wang, F.-K. Guo, C. Hanhart, U.-G. Meißner, Q. Zhao, Y(4260) as the first $S$-wave open charm vector molecular state?, Phys.Rev. D90 (7) (2014) 074039. arXiv: 1310.2190, doi:10.1103/PhysRevD.90.074039.

[358] F.-K. Guo, C. Hanhart, U.-G. Meißner, Evidence that the $Y(4660)$ is a $f_{0}(980) \psi^{\prime}$ bound state, Phys.Lett. B665 (2008) 26-29. arXiv : 0803.1392, doi:10.1016/j.physletb.2008.05.057.

[359] C. Yuan, et al. (Belle collaboration), Observation of $e^{+} e^{-} \rightarrow K^{+} K^{-} J \psi$ via initial state radiation at Belle, Phys.Rev. D77 (2008) 011105 . arXiv:0709.2565, doi:10.1103/PhysRevD.77.011105.

[360] C. P. Shen, et al. (Belle collaboration), Updated cross section measurement of $e^{+} e^{-} \rightarrow K^{+} K^{-} J / \psi$ and $K_{S}^{0} K_{S}^{0} J / \psi$ via initial state radiation at Belle, Phys.Rev. D89 (7) (2014) 072015. arXiv:1402.6578, doi:10.1103/PhysRevD.89.072015.

[361] X. H. Mo, G. Li, C. Z. Yuan, K. L. He, H. M. Hu, et al., Determining the upper limit of $\Gamma_{e e}$ for the Y(4260), Phys.Lett. B640 (2006) $182-187$. arXiv:hep-ex/0603024, doi:10.1016/j.physletb.2006.07.060.

[362] X. Wang, Y. Han, C. Yuan, C. Shen, P. Wang (Belle collaboration), Observation of $\psi(4040)$ and $\psi(4160)$ decay into $\eta J / \psi$, Phys.Rev. D87 (5) (2013) 051101. arXiv: 1210.7550, doi:10.1103/PhysRevD.87.051101.

[363] Z. Q. Liu, X. S. Qin, C. Z. Yuan, Combined fit to BABAR and Belle data on $e^{+} e^{-} \rightarrow \pi^{+} \pi^{-} \psi(2 S)$, Phys.Rev. D78 (2008) 014032 . arXiv: 0805.3560, doi:10.1103/PhysRevD.78.014032.

[364] B. Aubert, et al. (BABAR collaboration), The $e^{+} e^{-} \rightarrow K^{+} K^{-} \pi^{+} \pi^{-}, K^{+} K^{-} \pi^{0} \pi^{0}$ and $K^{+} K^{-} K^{+} K^{-}$cross-sections measured with initial-state radiation, Phys.Rev. D76 (2007) 012008. arXiv:0704.0630, doi:10.1103/PhysRevD.76.012008.

[365] B. Aubert, et al. (BABAR collaboration), Measurements of $e^{+} e^{-} \rightarrow K^{+} K^{-} \eta, K^{+} K^{-} \pi^{0}$ and $K_{s}^{0} K^{ \pm} \pi^{\mp}$ cross- sections using initial state radiation events, Phys.Rev. D77 (2008) 092002. arXiv:0710.4451, doi:10.1103/PhysRevD.77.092002.

[366] B. Aubert, et al. (BABAR collaboration), Study of the Exclusive Initial-State Radiation Production of the DD System, Phys.Rev. D76 (2007) 111105. arXiv: hep-ex/0607083, doi:10.1103/PhysRevD.76.111105.

[367] B. Aubert, et al. (BABAR collaboration), Exclusive Initial-State-Radiation Production of the $D \bar{D}, D^{*} \bar{D}$ and $D^{*} \bar{D}^{*}$ Systems, Phys.Rev. D79 
(2009) 092001. arXiv:0903.1597, doi:10.1103/PhysRevD.79.092001.

[368] D. Cronin-Hennessy, et al. (CLEO collaboration), Measurement of Charm Production Cross Sections in $e^{+} e^{-}$Annihilation at Energies between 3.97 and 4.26-GeV, Phys.Rev. D80 (2009) 072001. arXiv:0801.3418, doi :10.1103/PhysRevD.80.072001.

[369] P. del Amo Sanchez, et al. (BABAR collaboration), Exclusive Production of $D_{s}^{+} D_{s}^{-}, D_{s}^{*+} D_{s}^{-}$, and $D_{s}^{*+} D_{s}^{*-}$ via $e^{+} e^{-}$Annihilation with InitialState-Radiation, Phys.Rev. D82 (2010) 052004. arXiv: 1008.0338, doi : 10.1103/PhysRevD . 82. 052004.

[370] G. Pakhlova, et al. (Belle collaboration), Measurement of the $e^{+} e^{-} \rightarrow D^{0} D^{*-} \pi^{+}$cross section using initial-state radiation, Phys.Rev. D80 (2009) 091101. arXiv:0908.0231, doi:10.1103/PhysRevD.80.091101.

[371] T. Pedlar, et al. (CLEO collaboration), Observation of the $h_{c}(1 P)$ using $e^{+} e^{-}$collisions above $D \bar{D}$ threshold, Phys.Rev.Lett. 107 (2011) 041803. arXiv: 1104.2025, doi:10.1103/PhysRevLett.107.041803.

[372] B. Aubert, et al. (BABAR collaboration), A Study of $e^{+} e^{-} \rightarrow p \bar{p}$ using initial state radiation with BABAR, Phys.Rev. D73 (2006) 012005 . arXiv:hep-ex/0512023, doi:10.1103/PhysRevD.73.012005.

[373] B. Aubert, et al. (BABAR collaboration), Evidence of a broad structure at an invariant mass of $4.32 \mathrm{GeV} / c^{2}$ in the reaction $e^{+} e^{-} \rightarrow \pi^{+} \pi^{-} \psi(2 S)$ measured at BABAR, Phys.Rev.Lett. 98 (2007) 212001. arXiv: hep-ex/0610057, doi:10.1103/PhysRevLett.98.212001.

[374] M. Ablikim, et al. (BES III collaboration), Observation of $e^{+} e^{-} \rightarrow \omega \chi_{c 1,2}$ near $\sqrt{s}=4.42$ and $4.6 \mathrm{GeV}$, Phys.Rev. D93 (1) (2016) 011102. arXiv: 1511.08564, doi:10.1103/PhysRevD.93.011102.

[375] X. Li, M. B. Voloshin, $\chi_{c 0} \omega$ production in $e^{+} e^{-}$annihilation through $\psi(4160)$, Phys.Rev. D91 (3) (2015) 034004. arXiv:1411.2952, doi:10.1103/PhysRevD.91.034004.

[376] S.-K. Choi, et al. (Belle collaboration), Observation of a resonance-like structure in the $\pi^{ \pm} \psi^{\prime}$ mass distribution in exclusive $B \rightarrow K \pi^{ \pm} \psi^{\prime}$ decays, Phys.Rev.Lett. 100 (2008) 142001. arXiv:0708.1790, doi:10.1103/PhysRevLett.100.142001.

[377] R. Aaij, et al. (LHCb collaboration), Model-independent confirmation of the Z(4430) ${ }^{-}$state, Phys.Rev. D92 (11) (2015) 112009, [Phys.Rev.D92,112009(2015)]. arXiv:1510.01951, doi:10.1103/PhysRevD.92.112009.

[378] T. Aaltonen, et al. (CDF collaboration), Evidence for a Narrow Near-Threshold Structure in the $J \psi \phi$ Mass Spectrum in $B^{+} \rightarrow J \psi \phi K^{+}$ Decays, Phys.Rev.Lett. 102 (2009) 242002. arXiv:0903.2229, doi:10.1103/PhysRevLett.102.242002.

[379] T. Aaltonen, et al. (CDF collaboration), Observation of the $Y(4140)$ structure in the $J \psi \phi$ Mass Spectrum in $B^{ \pm} \rightarrow J \psi \phi K$ decays (2011) arXiv: 1101.6058

[380] J. Brodzicka, Heavy Flavour Spectroscopy, Conf. Proc. C0908171 (2009) 299-311, [,299(2009)]. doi:10.3204/DESY-PROC-2010-04/ 38.

[381] R. Aaij, et al. (LHCb collaboration), Search for the X(4140) state in $B^{+} \rightarrow J \psi \phi K^{+}$decays, Phys.Rev. D85 (2012) 091103 . arXiv: 1202.5087, doi:10.1103/PhysRevD.85.091103.

[382] V. M. Abazov, et al. (D $\varnothing$ collaboration), Search for the $X(4140)$ state in $B^{+} \rightarrow J \psi \phi K^{+}$decays with the D $\varnothing$ detector, Phys.Rev. D89 (1) (2014) 012004. arXiv: 1309.6580, doi:10.1103/PhysRevD.89.012004.

[383] J. Lees, et al., Study of $B^{ \pm, 0} \rightarrow J \psi K^{+} K^{-} K^{ \pm, 0}$ and search for $B^{0} \rightarrow J \psi \phi$ at BABAR, Phys.Rev. D91 (1) (2015) 012003. arXiv:1407.7244, doi:10.1103/PhysRevD.91.012003.

[384] V. M. Abazov, et al. (D $\varnothing$ collaboration), Inclusive Production of the X(4140) State in $p \bar{p}$ Collisions at D $\varnothing$, Phys.Rev.Lett. 115 (23) (2015) 232001. arXiv:1508.07846, doi:10.1103/PhysRevLett.115.232001.

[385] T. Branz, T. Gutsche, V. E. Lyubovitskij, Hadronic molecule structure of the $Y(3940)$ and $Y(4140)$, Phys.Rev. D80 (2009) 054019. arXiv: 0903.5424, doi:10.1103/PhysRevD.80.054019.

[386] M. Ablikim, et al. (BESIII collaboration), Search for the $Y(4140)$ via $e^{+} e^{-} \rightarrow \gamma \phi J \psi$ at $\sqrt{s}=4.23,4.26$ and $4.36 \mathrm{GeV}$, Phys.Rev. D91 (3) (2015) 032002. arXiv: 1412.1867, doi:10.1103/PhysRevD.91.032002.

[387] R. Aaij, et al. (LHCb collaboration), Model-independent evidence for $J / \psi p$ contributions to $\Lambda_{b}^{0} \rightarrow J / \psi p K^{-}$decays, Phys.Rev.Lett. 117 (8) (2016) 082002. arXiv: 1604.05708, doi:10.1103/PhysRevLett.117.082002.

[388] R. Aaij, et al. (LHCb collaboration), Evidence for exotic hadron contributions to $\Lambda_{b}^{0} \rightarrow J / \psi p \pi^{-}$decays, Phys.Rev.Lett. 117 (8) (2016) 082003, [Addendum: Phys. Rev. Lett.117,no.10,109902(2016)]. arXiv:1606.06999, doi:10.1103/PhysRevLett.117.082003.

[389] V. V. Anisovich, M. A. Matveev, J. Nyiri, A. V. Sarantsev, A. N. Semenova, Pentaquarks and resonances in the $p J / \psi$ spectrum (2015). arXiv: 1507.07652 .

[390] R. F. Lebed, The Pentaquark Candidates in the Dynamical Diquark Picture, Phys.Lett. B749 (2015) 454-457. arXiv: 1507.05867, doi : $10.1016 / j$.physletb.2015.08.032.

[391] L. Roca, J. Nieves, E. Oset, LHCb pentaquark as a $\bar{D}^{*} \Sigma_{c}-\bar{D}^{*} \Sigma_{c}^{*}$ molecular state, Phys.Rev. D92 (9) (2015) 094003. arXiv: 1507.04249, doi:10.1103/PhysRevD.92.094003.

[392] Q. Wang, X.-H. Liu, Q. Zhao, Photoproduction of hidden charm pentaquark states $P_{c}^{+}(4380)$ and $P_{c}^{+}(4450)$, Phys.Rev. D92 (2015) 034022. arXiv: 1508.00339, doi:10.1103/PhysRevD.92.034022.

[393] A. N. Hiller Blin, C. Fernández-Ramírez, A. Jackura, V. Mathieu, V. I. Mokeev, A. Pilloni, A. P. Szczepaniak, Studying the $P_{c}(4450)$ resonance in $J / \psi$ photoproduction off protons, Phys.Rev. D94 (3) (2016) 034002. arXiv: 1606.08912, doi:10.1103/PhysRevD.94. 034002.

[394] J. Brodzicka, et al. (Belle collaboration), Observation of a new $D_{s J}$ meson in $B^{ \pm} \rightarrow \bar{D}^{0} D^{0} K^{+}$decays, Phys.Rev.Lett. 100 (2008) 092001. arXiv:0707.3491, doi:10.1103/PhysRevLett.100.092001.

[395] T. Barnes, S. Godfrey, E. Swanson, Higher charmonia, Phys.Rev. D72 (2005) 054026. arXiv:hep-ph/0505002, doi:10.1103/ PhysRevD.72.054026.

[396] F.-K. Guo, U.-G. Meißner, Where is the $\chi_{c 0}(2 P)$ ?, Phys.Rev. D86 (2012) 091501. arXiv:1208.1134, doi:10.1103/PhysRevD.86. 091501.

[397] S. L. Olsen, Is the $X(3915)$ the $\chi_{c 0}(2 P)$ ?, Phys.Rev. D91 (5) (2015) 057501. arXiv: 1410.6534, doi:10.1103/PhysRevD.91.057501.

[398] X. Li, M. B. Voloshin, $X(3915)$ as a $D_{s} \bar{D}_{s}$ bound state, Phys.Rev. D91 (11) (2015) 114014. arXiv: 1503.04431, doi:10.1103/PhysRevD. 91.114014.

[399] CMS collaboration, Search for the $X(5568)$ state in $B_{s}^{0} \pi^{ \pm}$decays, CMS-PAS-BPH-16-002 (2016). 
[400] A. Ali, L. Maiani, A. D. Polosa, V. Riquer, B $c_{c}^{ \pm}$decays into tetraquarks, Phys.Rev. D94 (3) (2016) 034036. arXiv:1604.01731, doi: 10.1103/PhysRevD.94.034036.

[401] T. J. Burns, E. S. Swanson, Interpreting the X(5568), Phys.Lett. B760 (2016) 627-633. arXiv: 1603.04366, doi:10.1016/j ·physletb. 2016.07 .049$.

[402] F.-K. Guo, U.-G. Meißner, B.-S. Zou, How the X(5568) challenges our understanding of QCD, Commun.Theor.Phys. 65 (5) (2016) 593-595. arXiv: 1603.06316, doi:10.1088/0253-6102/65/5/593.

[403] J. L. Rosner, Hadron spectroscopy: A 2005 snapshot, AIP Conf.Proc. 815 (2006) 218-232, [,218(2005)]. arXiv:hep-ph/0508155, doi:10.1063/1.2173591.

[404] K.-T. Chao, Interpretations for the X(4160) observed in the double charm production at B factories, Phys.Lett. B661 (2008) 348-353. arXiv:0707.3982, doi:10.1016/j.physletb.2008.02.039.

[405] C. Deng, J. Ping, H. Huang, F. Wang, Systematic study of $Z_{c}^{+}$family from a multiquark color flux-tube model, Phys.Rev. D92 (3) (2015) 034027. arXiv:1507.06408, doi:10.1103/PhysRevD.92.034027.

[406] G. Corcella, I. Knowles, G. Marchesini, S. Moretti, K. Odagiri, et al., HERWIG 6: An Event generator for hadron emission reactions with interfering gluons (including supersymmetric processes), JHEP 0101 (2001) 010. arXiv: hep-ph/0011363, doi : 10.1088/1126-6708/ 2001/01/010.

[407] T. Sjostrand, S. Mrenna, P. Z. Skands, PYTHIA 6.4 Physics and Manual, JHEP 0605 (2006) 026. arXiv: hep-ph/0603175, doi:10. 1088/1126-6708/2006/05/026.

[408] M. Goldberger, K. Watson, Collision Theory, Dover books on physics, Dover Publications, 2004. URL https : //books . google. com/books?id=4pnrHN8Y1AgC

[409] D. Acosta, et al. (CDF collaboration), Measurement of prompt charm meson production cross sections in $p \bar{p}$ collisions at $\sqrt{s}=1.96$ TeV , Phys.Rev.Lett. 91 (2003) 241804. arXiv: hep-ex/0307080, doi:10.1103/PhysRevLett.91.241804.

[410] U. Fano, Effects of Configuration Interaction on Intensities and Phase Shifts, Phys.Rev. 124 (1961) 1866-1878. doi:10.1103/PhysRev . 124.1866.

[411] H. Feshbach, A Unified theory of nuclear reactions. 2., Annals Phys. 19 (1962) 287-313, [Annals Phys.281,519(2000)]. doi:10.1016/ 0003-4916(62) 90221-X. 